Harmonics Suppression of Vacuum Chamber Eddy Current Induced Fields With Application to the Superconducting Super Collider (SSC) Low Energy Booster (LEB) Magnets*

\author{
R. D. Schlueter
}

\author{
Advanced Light Source \\ Accelerator and Fusion Research Division \\ Lawrence Berkeley Laboratory \\ University of California \\ Berkeley, CA 94720
}

December 4, 1991

This work was supported by the Director, Office of Energy Research, Office of Basic Energy Sciences, Materials Sciences Division of the U.S. Department of Energy, under Contract No. DE-AC03-76SF00098 


\title{
Harmonics Suppression of Vacuum Chamber Eddy Current Induced Fields with Application to the Superconducting Super Collider (SSC) Low Energy Booster (LEB) Magnets
}

\author{
Ross D. Schlueter and Klaus Halbach
}

December 4, 1991

\begin{abstract}
This memo presents the formulation of an expression for eddy currents induced in a thinwalled conductor due to a time-dependent electromagnet field excitation. Then follows an analytical development for prediction of vacunm chamber eddy current induced field harmonics in iron-core electromagnets. A passive technique for harmonics suppression is presented with specific application to the design of the Superconducting Super Collider (SSC) Low Energy Booster (LEB) Magnets.
\end{abstract}

\section{Summary}

In Section 2 an expression. Eqn. (11), is derived relating the current density per unit length along the cross section of a conductor (e.g. a vacuum chamber) in a magnécic field to the vector potential of the field over that conductor's cross section.

Section 3 uses a Schwarz-Christoffel transformation to formulate an expression, Eqn. (21) or Eqn. (24), for field harmonics in an iron-core dipole electromagnet induced by arbitrarily located current filaments.

Section 4 shows that the chosen 'alternative' LEB dipole excitation pattern produces eddy current induced harmonics that are less than half that of a slightly different 'strawman' excitation pattern. A closed-form expression. Eqn. (33), for vacuum chamber eddy current induced harmonics in an iron-core dipole electromagnet is formulated using results from Sections 2 and 3. Analytical results of field harmonics from Code EDDY (Appendix C) for an elliptical strawman dipole magnet vacuum chamber shape are shown to match those of a lengthy two-step pair of POISSON runs. An expression, Eqn. (36), for the field correction (i.e. harmonics suppression) provided by a pair of passive compensating coils of given location and size in the dipole magnet field is developed. A candidate vacuum chamber shape, \# D of Figure 8 , is shown to reduce both the eddy current induced dipole component and the required maximum current in passive compensating coils by a factor of 4.6 over that of the strawman vacuum chamber design. The induced sextupole component is similarly reduced by a factor of 3.5. Recommended size and positioning of passive compensating coils capable of nulling both dipole and sextupole components are listed in Section 4.2.5. A $1 \mathrm{~mm}$ error in the positioning of the compensating coils gives rise to a 
field quality error of $1 \cdot 10^{-4}$ over $r=2.5 \mathrm{~cm}$. A coil positioning tolerance of $\sim 0.25 \mathrm{~mm}$ (10 mils) would put dipole magnet vacuum chamber eddy current induced harmonics in the noise with respect to the desired field quality.

In Section 5 a quadrupole magnet and vacuum chamber are mapped to dipole geometry, after which a closed-form expression. Eqn. (42), for vacuum chamber eddy current induced harmonics is formulated using results from Sections 2 and a Schwarz-Christoffel technique analogous to that in Section 3. Analytical results of field harmonics from Code EDDY (Appendix C) for an elliptical strawman quadrupole vacuum chamber shape are shown to match those of a lengthy two-step pair of POISSON ${ }^{[1]}$ runs. A candidate circular vacuum chamber shape, \# F of Figure 14, featuring a $4.0 \mathrm{~cm}$ ID circular vacuum chamber is shown to give a maximum quadrupole harmonic of $0.5 \cdot 10^{-3} B_{I_{\text {coil }}}$ at $|r|=2.5 \mathrm{~cm}$ (= $0.8 \cdot 10^{-3} B_{I_{\text {coil }}}$ at $\left.|r|=4.0 \mathrm{~cm}\right)$. All higher order harmonics are negligible. The magnitude of the vacuum chamber eddy current induced quadrupole term for these vacuum chamber shapes may be sufficiently low depending on the final dipole-quadrupole tracking design specification and tolerance to risk; correction coils may not be needed in the quadrupole magnet.

In Section 6 a generalized closed-form expression for vacuum chamber eddy current induced harmonics in an $m^{\text {th }}$ order multipole of 'symmetrical' vacuum chamber shape is formulated [Eqn. (44) or Eqn. (47)]. Generalized passive eddy current induced harmonics suppression in an $m^{\text {th }}$ order multipole is discussed. Pertinent generalized vacuum chamber shapes in combination with $\mathrm{m}^{\text {th }}$ order multipole magnets are summarized along with allowed harmonics. Correction coils are not needed in the LEB sextupole magnets.

\section{Eddy Currents in a Thin-walled Conductor}

If in an electromagnet with a sinusoidal time-dependent excitation

$$
I_{\text {coil }}=I_{D C}+I_{\Delta} \cos \omega t
$$

the iron yoke remains far from saturation, then, neglecting hysteresis, the two-dimensional magnetic vector potential $A$ in the good field region is very nearly linear with current:

$$
A\left(I_{\text {coil }}, x, y\right)=A\left(I_{D C}, x, y\right)+A\left(I_{\Delta}, x, y\right) \cos \omega t
$$

and the rate of change of the vector potential with time is

$$
\frac{d A\left(I_{\text {coil }}, x, y\right)}{d t}=-A\left(I_{\Delta}, x, y\right) \omega \sin \omega t
$$

Since $B \equiv \nabla \times A$, we can relate the electric field $E$ to the vector potential via Maxwell:

$$
-\nabla \times E=\frac{d B}{d t}=\nabla \times \frac{d A}{d t}
$$


from which it follows

$$
-E=\frac{d A}{d t}+c_{0}
$$

Eddy currents will be induced in any conducting medium present (such as a vacuum chamber), which alter the magnetic field in the region of interest. The current density in the conducting medium is given by:

$$
j=\sigma E=-\sigma\left(\frac{d A}{d t}+c_{0}\right)
$$

For a vacuum chamber of thickness $D$, the current per unit length along the arc $s$ of the conducting chamber is

$$
I_{v a c}^{\prime}(s \in z)=j D=-\sigma D\left(\frac{d A}{d t}+c_{0}\right)
$$

Since there is no net current flowing over the cross section of the conducting medium,

$$
\int_{s}-\sigma D\left(\frac{d A}{d t}+c_{0}\right) d s=0
$$

where $d s$ is the incremental arc length of the perimeter of the vacuum chamber. Thus $c_{0}$ is given by:

$$
c_{0}=\frac{-\int_{s} \sigma D \frac{d A}{d t} d s}{\int_{s} \sigma D d s}
$$

and we have for a uniformly thick and uniformly conductive vacuum chamber:

$$
\begin{gathered}
I_{v a c}^{\prime}(s \in z)=-\sigma D \omega \sin \omega t\left[-A\left(I_{\Delta}, s\right)+\frac{\int_{s} \sigma D A\left(I_{\Delta}, s\right) d s}{\int_{s} \sigma D d s}\right]= \\
\sigma D \omega \sin \omega t\left[A\left(I_{\Delta}, s\right)-\frac{1}{S} \int_{s} A\left(I_{\Delta}, s\right) d s\right]
\end{gathered}
$$

where $S$ is the perimeter of the vacuum chamber.

For a given magnet geometry, POISSON need be run for only one arbitrary magnet excitation, say $I_{\text {coil }}=I_{1}$, resulting in a tabulated vector potential distribution $A\left(I_{1}, x, y\right)$. Since the problem is linear, $\frac{I_{\Delta}}{I_{1}}=\frac{A\left(I_{\Delta}, x, y\right)}{A\left(I_{1}, x, y\right)}=\frac{B\left(I_{\Delta}, x, y\right)}{B\left(I_{1}, x, y\right)}$ and $I_{v a c}^{\prime}(s \in(x, y))$ can be expressed in terms of the tabulated vector potential $A\left(I_{1}, s\right)$ along the vacuum chamber contour, for any specified $I_{\Delta}$ (or equivalently, $B_{\Delta}$ ):

$$
I_{\text {vac }}^{\prime}(s \in(x, y), t)=\sigma D \omega \sin \omega t\left[\frac{I_{\Delta}}{I_{1}}\right]\left[A\left(I_{1}, s\right)-\frac{1}{S} \int_{s} A\left(I_{1}, s\right) d s\right]
$$



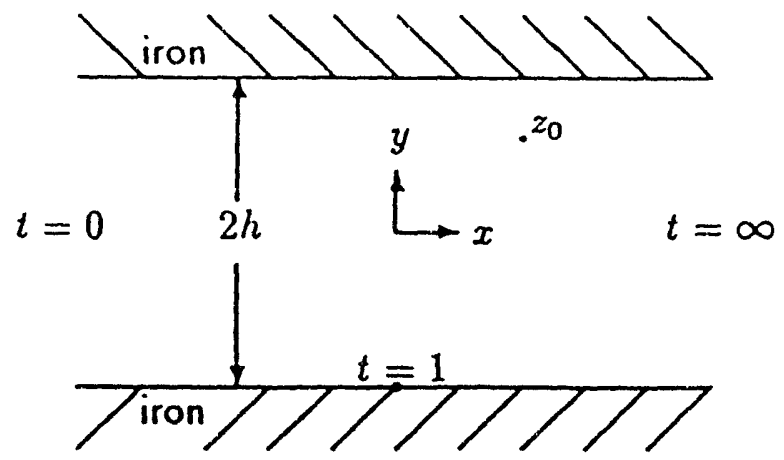

Figure 1a. Electromagnet dipole $z$ plane geometry

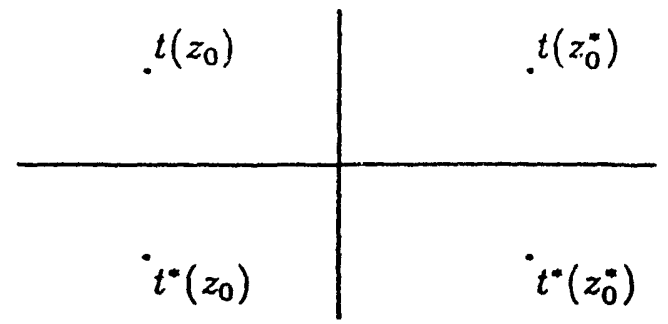

Figure 1b. Schwarz-Christoffel transformed $t$ plane geometry

\section{Harmonic Components of Field Perturbations Induced by Arbitrarily Located Current Filaments}

Lee ${ }^{[2]}$ has developed expressions for a multipole expansion of the field in a dipole magnet from vacuum chamber eddy currents. Here we formulate analytical expressions for field harmonics in a dipole electromagnet induced by arbitrarily located current filaments. Analogous expressions for higher order multipole magnets are obtainable after conformally mapping the multipole geometry to a dipole geometry.

Figure la shows the gap portion of a wide [in the $x$-direction] electromagnet dipole exhibiting midplane symmetry. Assuming the vacuum chamber also exhibits midplane symmetry, then current filaments of magnitude $I$ can be thought to occur in pairs at $z_{0}$ and $z_{0}^{*}$. The return paths for the currents will be $-I$ at $-z_{0}$ and $-z_{0}^{*}$ if the vacuum chamber is also symmetric about the $y$-axis.

The $x$-direction width of the iron pole face yoke is assumed effectively infinite in the analytical model. Exponential decay of field errors as one moves towards the origin from the corner of the iron pole insures that this approximation will not result in large errors of calculated harmonic components near the beam axis (i.e. the origin in Figure 1a).

We shall use a Schwarz-Christoffel transformation to conformally map the gap region between $z=(x, \pm h)$ of Figure la to the upper half $t$ plane shown in Figure 1b. ${ }^{1}$ Choosing $t=\infty$ at $z=+\infty$ results in the transformation

$$
\frac{d z}{d t}=\frac{1 / k}{t-a}
$$

Choosing $t=a=0$ at $z=-\infty$ makes $1 / k$ take on the value $\frac{2 h}{\pi}$ since at $t=0$, $\operatorname{Im}(\Delta z)=\operatorname{Im}\left(\oint_{t=0} \frac{1}{k t} d t\right), \longrightarrow 2 h=\operatorname{Im}\left(\frac{\pi i}{k}\right)=\frac{\pi}{k}$. Integrating with respect to $t$ gives

\footnotetext{
'Alternatively, since the dipole exhibits midplane symmetry, mapping the hall-gap region between $y=0$ and $y=h$ of Figure ia to the upper half $t$ plane yields the same result.
} 


$$
z=\frac{2 h}{\pi} \ln t+c, \longrightarrow t=e^{\frac{\pi(z-c)}{2 h}}
$$

Choosing $t=1$ at $z=-i h$ makes $c$ take on the value $-i h$. The complete transformation is thus

$$
z=\frac{2 h}{\pi} \ln t-i h, \longrightarrow t=i e^{\frac{\pi z}{2 h}}=i e^{k z}=i e^{\alpha}
$$

where $k \equiv \frac{\pi}{2 h}$ and $\alpha \equiv k z$.

For a filament of magnitude $+I$ at $z_{0}$ and its image in the $t$ plane about the real axis, which makes the magnetic field perpendicular to the air-iron interface, the complex potential $F(t)$ is given by

$$
F(t)=\frac{-I}{2 \pi} \ln \left(t-t\left(z_{0}\right)\right)\left(t-t^{*}\left(z_{0}\right)\right)
$$

The magnetic field is related to the complex potential by $H^{*}(z)=i \frac{d F}{d z}$. For the case under consideration we have

$$
H^{*}(z)=i \frac{d F / d t}{d z / d t}=\frac{I k e^{\alpha}}{2 \pi i}\left[\frac{1}{e^{\alpha}-e^{\alpha_{0}}}+\frac{1}{e^{\alpha}+e^{\alpha_{0}^{*}}}\right]
$$

For current filaments of magnitude $+I$ at $z_{0}$ and $z_{0}^{*}$, and their images in the $t$ plane about the real axis, we have for $H^{*}(z)$ :

$$
H^{*}(z)=i \frac{d F / d t}{d z / d t}=\frac{I k}{2 \pi i} e^{\alpha}\left[\frac{1}{e^{\alpha}-e^{\alpha_{0}}}+\frac{1}{e^{\alpha}+e^{\alpha_{0}^{*}}}+\frac{1}{e^{\alpha}-e^{\alpha_{0}^{*}}}+\frac{1}{e^{\alpha}+e^{\alpha_{0}}}\right]
$$

where the relations $t\left(z_{0}\right)=i e^{\alpha_{0}}, t^{*}\left(z_{0}\right)=-i e^{\alpha_{0}^{*}}, t\left(z_{0}^{*}\right)=i e^{\alpha_{0}^{*}}$, and $t^{*}\left(z_{0}^{*}\right)=-i e^{\alpha_{0}}$ have been used. Simplifying, we have

$$
H^{*}(z)=\frac{I k}{2 \pi i}\left[\frac{e^{\alpha-\alpha_{0}}}{\operatorname{Sinh}\left(\alpha-\alpha_{0}\right)}+\frac{e^{\alpha-\alpha_{0}^{*}}}{\operatorname{Sinh}\left(\alpha-\alpha_{0}^{*}\right)}\right]
$$

We now decompose these expressions for $H^{*}(z)$ into harmonic components. Define $G(z) \equiv \frac{e^{z}}{\operatorname{Sinh}(z)}-1=\frac{1}{\operatorname{Tanh}(z)}$. Then, expanding $G$ in a power series,

$$
G\left(\alpha-\alpha_{0}\right)=\frac{1}{\operatorname{Tanh}\left(\alpha-\alpha_{0}\right)}=\Sigma_{0} \alpha^{n} a_{n}=a_{0}+a_{1} \alpha+a_{2} \alpha^{2}+\ldots
$$

where, since $G(z)$ is an odd function, we have 


$$
n ! a_{n}=\left.\frac{\partial^{n}}{\partial \alpha^{n}}\left(\frac{1}{\operatorname{Tanh}\left(\alpha-\alpha_{0}\right)}\right)\right|_{\alpha=0}=(-1)^{n+1} \frac{\partial^{n}}{\partial \alpha_{0}^{n}}\left(\frac{1}{\operatorname{Tanh}\left(\alpha_{0}\right)}\right)=(-1)^{n+1} \frac{\partial^{n} G\left(\alpha_{0}\right)}{\partial \alpha_{0}^{n}}
$$

Therefore,

$$
\begin{gathered}
G\left(\alpha-\alpha_{0}\right)+G\left(\alpha-\alpha_{0}^{*}\right)=\Sigma_{0} \alpha^{n}\left(a_{n}+c_{n}\right)=\Sigma_{0} \frac{-1^{n+1} \alpha^{n}}{n !}\left[\frac{\partial^{n} G\left(\alpha_{0}\right)}{\partial \alpha_{0}^{n}}+\frac{\partial^{n} G\left(\alpha_{0}^{*}\right)}{\partial \alpha_{0}^{* n}}\right]= \\
\Sigma_{0} \frac{-1^{n+1} \alpha^{n}}{n !} 2 \operatorname{Re}\left[\frac{\partial^{n} G\left(\alpha_{0}\right)}{\partial \alpha_{0}^{n}}\right]
\end{gathered}
$$

Thus, the multipole components for a pair of current filaments of magnitude $+I$ at $z_{0}$ and $z_{0}^{*}$ are given by (dropping the uniform constant field):

$$
H^{*}(z)=\frac{I k}{2 \pi i}\left[G\left(\alpha-\alpha_{0}\right)+G\left(\alpha-\alpha_{0}^{*}\right)\right]=\frac{I}{2 h i} \Sigma_{0} \frac{-1^{n+1} \alpha^{n}}{n !} \operatorname{Re}\left[b_{n}\right]
$$

where

$$
\begin{gathered}
b_{0}=\left(\frac{1}{T}\right), \quad b_{1}=\left(\frac{-1}{S^{2}}\right), \quad b_{2}=\left(\frac{2 C}{S^{3}}\right), \\
b_{3}=(-2)\left(\frac{2 S^{2}+3}{S^{4}}\right), \quad b_{4}=\left(\frac{8 C}{S^{5}}\right)\left(S^{2}+3\right), \quad b_{n}=\frac{\partial^{n} G\left(\alpha_{0}\right)}{\partial \alpha_{0}^{n}}
\end{gathered}
$$

and where $S \equiv \operatorname{Sinh}\left(\alpha_{0}\right), C \equiv \operatorname{Cosh}\left(\alpha_{0}\right), T \equiv \operatorname{Tanh}\left(\alpha_{0}\right), \alpha \equiv \frac{\pi z}{2 h}$, and $\alpha_{0} \equiv \frac{\pi z_{0}}{2 h}$.

If additionally, the vacuum chamber is symmetric about the $y$-axis, the return paths for the currents will be $-I$ at $-z_{0}$ and $-z_{0}^{*}$.

We have for the $z$ location $-\alpha_{0}$ :

$$
G\left(\alpha+\alpha_{0}\right)=\frac{1}{\operatorname{Tanh}\left(\alpha+\alpha_{0}\right)}=\Sigma_{0} \alpha^{n} a_{n}=a_{0}+a_{1} \alpha+a_{2} \alpha^{2}+\ldots
$$

where

$$
n ! \alpha_{n}=\left.\frac{\partial^{n}}{\partial \alpha^{n}}\left(\frac{1}{\operatorname{Tanh}\left(\alpha+\alpha_{0}\right)}\right)\right|_{\alpha=0}=\frac{\partial^{n}}{\partial \alpha_{0}^{n}}\left(\frac{1}{\operatorname{Tanh}\left(\alpha_{0}\right)}\right)=\frac{\partial^{n} G\left(\alpha_{0}\right)}{\partial \alpha_{0}^{n}}
$$

Therefore,

$$
\begin{aligned}
G\left(\alpha+\alpha_{0}\right)+G\left(\alpha+\alpha_{0}^{*}\right)= & \Sigma_{0} \alpha^{n}\left(a_{n}+c_{n}\right)=\Sigma_{0} \frac{\alpha^{n}}{n !}\left[\frac{\partial^{n} G\left(\alpha_{0}\right)}{\partial \alpha_{0}^{n}}+\frac{\partial^{n} G\left(\alpha_{0}^{*}\right)}{\partial \alpha_{0}^{* n}}\right]= \\
& \Sigma_{0} \frac{\alpha^{n}}{n !} 2 \operatorname{Re}\left[\frac{\partial^{n} G\left(\alpha_{0}\right)}{\partial \alpha_{0}^{n}}\right]
\end{aligned}
$$




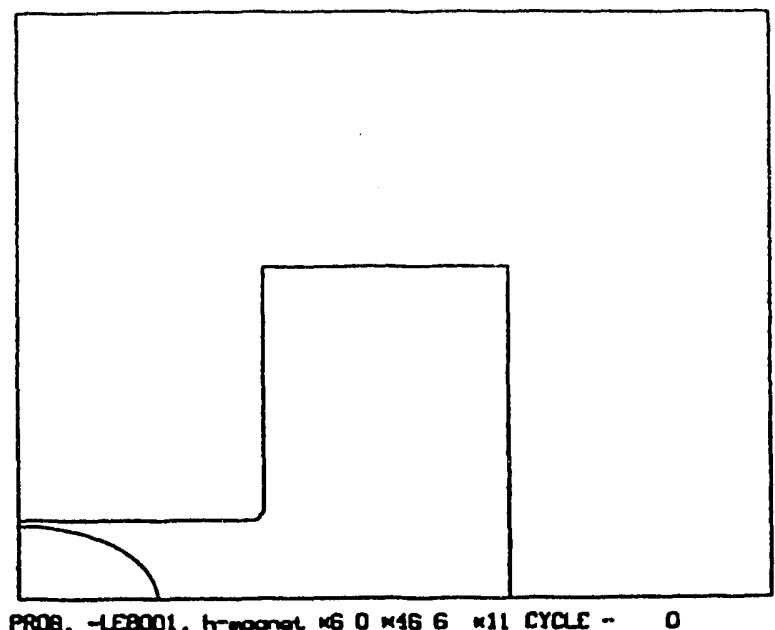

Figure 2. SSC Low Energy Booster strawman dipole magnet geometry.

Thus, for this special case with current filaments of magnitude $+I$ at $z_{0}$ and $z_{0}^{*}$ and $-I$ at $-z_{0}$ and $-z_{0}^{*}$, the multipole components are given by

$$
H^{*}(z)=\frac{I k}{\pi i} \Sigma_{0} \frac{-2 \alpha^{n}}{n !} \operatorname{Re}\left[b_{n}\right]=\frac{I}{h i} \Sigma_{n=e v e n} \frac{-\alpha^{n}}{n !} \operatorname{Re}\left[b_{n}\right]
$$

where the $b_{n}$ are as given above and where the $n=$ odd terms drop out.

\section{SSC Low Energy Booster Dipole Magnet Design}

The strawman geometry for the SSC low energy booster dipole magnet is shown in Figure 2. The near on-axis field has components due to both the magnet coil excitation and the eddy current excitation in the vacuum chamber:

$$
B_{\text {tot }}^{*}(z)=B_{I_{\text {coil }}}^{*}(z)+B_{I_{\text {tac }}^{\prime}(s)}^{*}(z)
$$

each of which may be decomposed into harmonics ${ }^{2}$ as expressed by:

$$
i B^{*}(z)=B_{0} \sum_{n=0}\left(b_{n}+i a_{n}\right) z^{n}
$$

The strawman field quality specification for these magnets are that $\frac{\Delta B}{B} \leq 10^{-4}$ at $|z|=2.5 \mathrm{~cm}$ (later changed to $\frac{\Delta B}{B} \leq 10^{-4}$ at $|z| \leq 3.25 \cos \theta+i 2.5 \sin \theta \mathrm{cm}$.) The

\footnotetext{
${ }^{2}$ Here we use SSC's harmonics nomenclature; POISSON code results are generated using the convention $B^{\circ}(z)=$ $\sum_{n=1}$ in $\frac{\left(a_{n}+i b_{n}\right)}{r}\left(\frac{z}{r}\right)^{n-1}$, thus for the $k^{\text {th }}$ harmonic $\left(k=0\right.$ being the dipole) SSC's $B_{0} b_{k}$ and $B_{0} a_{k}$ are equivalent to POLSSON's (see Figure 4) $\frac{-(k+1) a_{k+1}}{r} \frac{1}{r^{k}}$ and $\frac{-(k+1) b_{k+1}}{r} \frac{1}{r^{k}}$, respectively.
} 
excitation is to produce a field strength that cycles between $0.13 \mathrm{~T}$ and $1.3 \mathrm{~T}$. The half gap is $2.86 \mathrm{~cm}$.

Maintaining the dipole quality of the first term of Eqn. (25) consists of designing the pole so as to avoid magnetic saturation and shaping the tip so as to compensate for the finite shape of the pole. The LEB dipole magnet design and harmonics tabulations for various magnet coil excitation's are discussed elsewhere ${ }^{[3,4]}$. Specifications are summarized in Appendix A.

Maintaining the dipole quality of the second term of Eqn. (25) consists of either shaping the vacuum chamber or adding compensating eddy current carrying conductor filaments so as to null harmonic components due to the eddy current distribution in the vacuum chamber conductor. Eddy currents in the vacuum chamber are insensitive to magnet design changes improving the quality of near on-axis field, thus field quality improvement modifications associated with the two contributions to the dipole given by Eqn. (25) may be decoupled.

\subsection{Suppression of Pole Magnet Geometry and Iron Saturation Induced Harmonics}

Harmonics suppression via designing the pole so as to avoid magnetic saturation and shaping the tip so as to compensate for the finite $x$-direction pole width is outside the principal thrust of this memo and is discussed elsewhere ${ }^{[3,4]}$.

\subsection{Suppression of Vacuum Chamber Eddy Current Induced Harmonics}

\subsubsection{Effect of Magnet Excitation Pattern on Maximum $\frac{\Delta B}{B}[5]$}

The field quality goal with repspect to the eddy current induced field perturbations is to minimize not the field perturbations themselves, but rather the ratio of the field perturbations to the total field $B_{\text {tot }}$. For a magnet of given geometry, it is interesting to see how minor changes of the excitation pattern can dramatically change the magnitude of the eddy current induced maximum $\frac{\Delta B}{B}$. Here excitation modification effects on harmonics are illustrated as an aide for those situations when one has such a freedom.

It should be noted that the ratio of the field harmonics (e.g dipole:quadrupole:sextupole) due to the eddy current distribution in the vacuum chamber is constant for a given chamber shape and does not change with magnet excitation. Thus when altering the excitation pattern for a given magnet and vacuum chamber geometry, the various harmonic components of eddy current induced field perturbations are altered proportionately. Also note that $\left|B_{I_{\text {coil }}}\right| \gg\left|B_{I_{\text {tac }}}\right|$ and thus $\left|B_{\text {tot }}\right| \simeq\left|B_{I_{\text {coil }}}\right|$.

The magnitude of $B_{I_{\text {vac }}}$ is proportional to the time rate of change of the field due to the magnet excitation:

$$
B_{I_{\text {vac }}^{\prime}(s)} \propto I_{\text {vac }}^{\prime}(s) \propto j \propto E \propto \frac{d A}{d t} \propto t B_{I_{\text {coil }}} / d t
$$




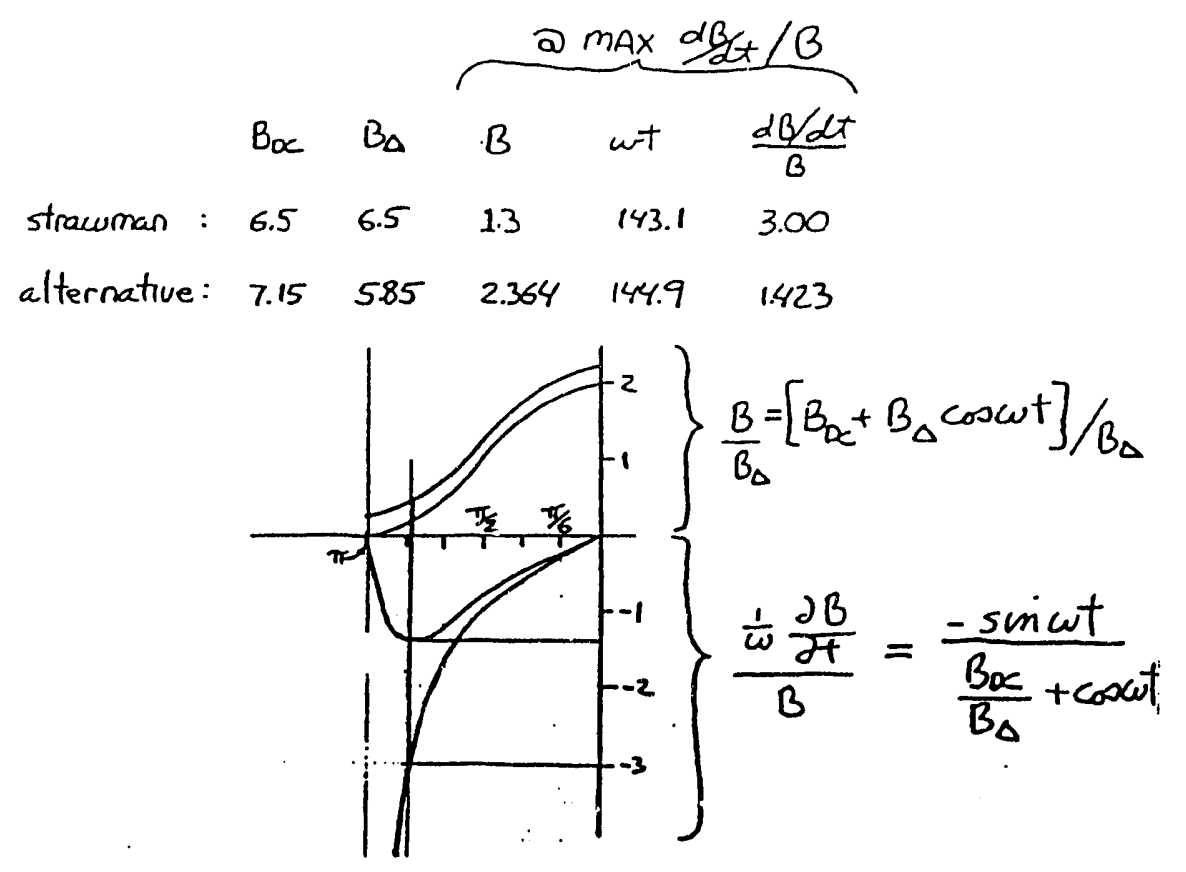

Figure 3. $\left|\frac{d B_{I_{\text {coil }}} / d t}{B_{I_{\text {coil }}}}\right|$ and $B$ versus $\omega t$ for two magnet excitation functions.

Thus, $\left|\frac{B_{I_{\text {cac }}}}{B_{\text {tor }}}\right|$ is largest when $\left|d B_{I_{\text {coid }}} / d t / B_{I_{\text {coil }}}\right|$ is maximized.

For the current excitation given by Eqn. (1), the $I_{\text {coil }}$ induced magnetic flux density in the good field region and its rate of change with time are given by the numerator and denominator, respectively, of Eqn. (28):

$$
\frac{d B_{I_{\text {coil }}} / d t}{B_{I_{\text {coil }}}}=\frac{-B_{\Delta} \omega \sin \omega t}{B_{d c}+B_{\Delta} \cos \omega t}
$$

At its maximum, we have:

$$
\left|\frac{d B_{I_{\text {coil }}} / d t}{B_{I_{\text {coil }}}}\right|_{\cos \omega t=-B_{\Delta} / B_{d c}}=\frac{\omega}{\sqrt{\left(\frac{B_{d c}}{B_{\Delta}}\right)^{2}-1}}
$$

Assume a 'strawman' dipole excitation design range were $0-13 \mathrm{kG}$, with injection at $1.3 \mathrm{kG}$. Thus $B_{d c}=B_{\Delta}=6.5 \mathrm{kG}$. $\left|\frac{d B / d t}{B}\right|$ versus $\omega t$ for these values is plotted in Figure 3 along with a plot for the for an 'alırnative' excitation pattern with a range $1.3-13.0$ $\mathrm{kG}$, and with injection at $1.3 \mathrm{kG}$ (i.e. $B_{\mathrm{d}:}=7.15 \mathrm{kG}$ and $B_{\Delta}=5.85 \mathrm{kG}$ ).

For the strawman excitation $\left(B_{d c}=B_{\Delta}=6.5 \mathrm{kG}\right)$, the maximum $\left|\frac{d B / d t}{B}\right|$ that the protons see is at injection, where the field is $1.3 \mathrm{kG}$ (see Figure 3 ). Here $\omega t \simeq 143.1^{\circ}$ and $\left|\frac{d B / d t}{B}\right|=3.00 \omega$. For the alternative excitation $\left(B_{d c}=7.15 \mathrm{kG}, B_{\Delta}=5.85 \mathrm{kG}\right)$, the maximum $\left|\frac{d B / d t}{B}\right|$ that the protons see is at $\omega t \simeq 144.9^{\circ}$ (see Figure 3 ). Here the field is $B=2.364 \mathrm{kG}$ and $\left|\frac{d B / d t}{B}\right|=1.423 \omega$. Thus, maximum $\frac{\Delta B}{B}$ due to eddy currents is reduced 
by $\sim$ half when the alternative excitation pattern is employed. The proposed excitation pattern for the LEB dipole magnets is that of the 'alternative' excitation pattern above.

\subsubsection{Numerical Adaptation of Vacuum Chamber Eddy Current Effects to POISSON}

It is convenient to model the continuously varying current sheet $I_{v a c}^{\prime}(s)$ of Eqn. (11) as discretized currents at $N$ mesh points along the vacuum chamber arc $s$ :

$$
d I_{s_{j}}=I_{s_{j}}^{\prime} d s_{j}
$$

where $d s_{j}=0.5\left(\left|\vec{z}_{j+1}-\vec{z}_{j}\right|+\left|\vec{z}_{j}-\vec{z}_{j-1}\right|\right)$ for $j=2, \ldots, N-1, d s_{1}=0.5\left|\vec{z}_{2}-\vec{z}_{1}\right|$, and $d s_{N}=0.5\left|\vec{z}_{N}-\vec{z}_{N-1}\right|$.

If we have Dirichlet vector potential symmetry about the $y$-axis, as in Figure 2 , $A(x, y)=-A(x,-y)$ and the integral term in Eqn. (11) vanishes. If we also have Neumann vector potential symmetry about the $x$-axis, as in Figure $2, A(x, y)=A(x,-y)$ and we can use the midplane symmetry plane default $(\operatorname{con}(22)=1)$ in POISSON.

Normalization of Eqn. (11) with respect to $\sigma D \frac{d B_{\lambda_{c o i l}}}{d t}$ and using $\frac{L_{A}}{I_{1}}=\frac{B_{A}}{B_{1}}$ gives for the input vacuum chamber current filaments in POISSON:

$$
\frac{d I_{s_{j}}}{\sigma D \frac{d B_{I_{c a i l}}}{d t}}=\frac{d I_{s_{j}}}{-\sigma D B_{\Delta} \omega \sin \omega t}=\left(-A\left(I_{1}, s_{j}\right)+\frac{1}{S} \int_{s} A\left(I_{1}, s\right) d s\right) \frac{d s_{j}}{B_{1}}
$$

The strawman LEB vacuum chamber is an ellipse with $x$ and $y$ major half-axes $a_{x}=5.5$ $\mathrm{cm}$ and $b_{y}=2.75 \mathrm{~cm}$, respectively. For this symmetry about the $y$-axis, with $A(0, y)=0$ the integral term in Eqn. (31) is zero. The eddy current induced harmonic components from a POISSON run with current filaments $I_{s_{j}}$ input according to Eqn. (31), with $\sigma D \frac{d B_{L_{\text {coil }}}}{d t}=1 \mathrm{~A} / \mathrm{cm}^{2}$ and with the $A\left(I_{1}, s_{j}\right), B_{1} \equiv i B^{*}(z)=B_{y}$, and $d s_{j}$ from the dipole magnet excitation POISSON run with $I_{\text {coil }}$ set equal to an arbitrary value $I_{1}$, as discussed above Eqn. (11), are tabulated in Figure 4. Sample POISSON files are found in Appendix B. POISSON results for eddy current induced harmonics for the LEB dipole magnet are summarized in Figure 5. The quadrupole, octupole, etc. terms are zero for this vacuum chamber exhibiting symmetry with respect to the $y$-axis.

4.2.3 Closed Form Analytical Prediction of Eddy Current Induced Harmonics for LEB Dipole Magnet

For a dipole magnet with a vacuum chamber symmetric about the $y$ axis, $\frac{A\left(I_{1}, x, y\right)}{B_{1}}=-x$. Thus from Eqn. (11) or (31), we have for the current sheet along the vacuum chamber:

$$
I_{v a c}^{\prime}(s, t)=x \sigma D \frac{d B_{I_{\text {coil }}}}{d t}
$$

Using the value for $I_{\text {vac }}^{\prime}(s, t)$ given by Eqn. (32) in the expression for eddy current induced harmonics Eqn. (21) yields 
TABLE FOR FIELD COEFFICIENTS

NORMALIZATION RADIUS $=\quad 2.50000$
$\begin{array}{cccc}(B X-I B Y)=I * S U M & N^{*}(A N+I B N) / R *(Z / R) * *(N-1) \\ N & N(A N) / R & N(B N) / R & A B S(N(C N) / R) \\ 1 & 8.5375 E+00 & 0.0000 E+00 & 8.5375 E+00 \\ 3 & -1.1488 E+00 & 0.0000 E+00 & 1.1488 E+00 \\ 5 & 5.6757 E-02 & 0.0000 E+00 & 5.6757 E-02 \\ 7 & 7.6619 E-03 & 0.0000 E+00 & 7.6619 \mathrm{E}-03 \\ 9 & -2.2467 E-03 & 0.0000 E+00 & 2.2467 E-03\end{array}$

Figure 4. Vacuum chamber eddy current induced magnetic field harmonics for strawman LEB dipole magnet design (POISSON output)

- input $I_{a^{\prime}{ }^{\prime}}$ per Eqn. (31),

- LEB dipole magnet geometry per Figure 2, half gap $h=2.86 \mathrm{~cm}$

- elliptical vacuum chamber with major half-axes of $5.5 \mathrm{~cm}$ and $2.75 \mathrm{~cm}$

At $|r|=2.5 \mathrm{~cm}$ :

\begin{tabular}{|c|c|c|c|c|c|c|}
\hline $\begin{array}{l}\text { harmonic } \\
\text { component }\end{array}$ & 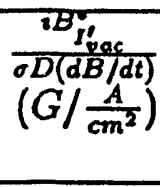 & 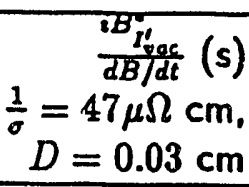 & \multicolumn{2}{|c|}{$\begin{array}{c}i B_{I_{\text {sac }}^{*}}^{*}(G) \\
\text { at condition \#1: } \\
\sigma D \frac{d B}{d t}=2.346 \frac{A}{\mathrm{c}^{2}}\end{array}$} & \multicolumn{2}{|c|}{$\begin{array}{c}i B_{l_{\text {soc }}^{*}}^{*}(G) \\
\text { at condition \#2: } \\
\sigma D \frac{d B}{d t}=1.349 \frac{A}{\mathrm{~cm}^{2}}\end{array}$} \\
\hline & & & (G) & $\left(\%\right.$ of $\left.B_{I_{\text {coil }}}\right)$ & (G) & $10 \%$ of $R_{1}$ \\
\hline $\begin{array}{l}\text { dipole: } \\
\text { sextupole: } \\
\text { decapole: }\end{array}$ & $\begin{array}{r}-8.5400 \\
1.1500 \\
-0.0567\end{array}$ & $\begin{array}{r}-54.5 \cdot 10^{-6} \\
7.34 \cdot 10^{-6} \\
-.362 \cdot 10^{-6}\end{array}$ & $\begin{array}{r}-20.00 \\
2.7030 \\
-.1330\end{array}$ & $\begin{array}{r}-.2800 \\
.0377 \\
-.0019\end{array}$ & $\begin{array}{r}-11.50 \\
1.550 \\
-.0765\end{array}$ & $\begin{array}{r}-.4865 \\
.0656 \\
-.0032\end{array}$ \\
\hline
\end{tabular}

where conditions \#1 and \#2 are defined by

- an excitation pattern: $\omega=20 \pi / \mathrm{s}, B_{d c}=7.15 \mathrm{kG}, B_{\Delta}=5.85 \mathrm{kG}$, and

- a vacuum chamber conductivity $\sigma, \frac{1}{\sigma}=47 \mu \Omega \mathrm{cm}$ and thickness $D=0.03 \mathrm{~cm}$

\begin{tabular}{|c|c|c|c|c|c|c|}
\hline condition \# & condition & $\omega t$ & $\begin{array}{l}B_{I_{\text {coil }}} \\
(\mathrm{G})\end{array}$ & $\begin{array}{c}d B_{I_{\text {coil }} / d t} / d t \\
(\mathrm{G} / \mathrm{s})\end{array}$ & $\begin{array}{c}\frac{d B_{I_{\text {cai }}} / d t}{B_{\text {I }_{\text {coil }}}} \\
(\mathrm{s})\end{array}$ & $\begin{array}{c}\sigma D \frac{d B_{l_{\text {cail }}}}{d t}\left(\frac{A}{\mathrm{~cm}^{2}}\right) \\
\text { at } D=0.03 \mathrm{~cm} \text {, } \\
\frac{1}{\sigma}=47 \mu \Omega \mathrm{cm}\end{array}$ \\
\hline 1 & $B_{I_{\text {tac }}^{\prime}}$ maximized & $90^{\circ}$ & 7150 & 367,566 & 51.4 & 2.346 \\
\hline 2 & $\frac{B_{t_{\text {tac }}^{\prime}}}{B_{\text {enc }}}$ maximized & $144.9^{\circ}$ & 2364 & 211,368 & 89.4 & 1.349 \\
\hline
\end{tabular}

Figure 5. LEB dipole magnet eddy current induced harmonics from POISSON 
Figure 6. LEB dipole magnet eddy current induced harmonics (analytical)

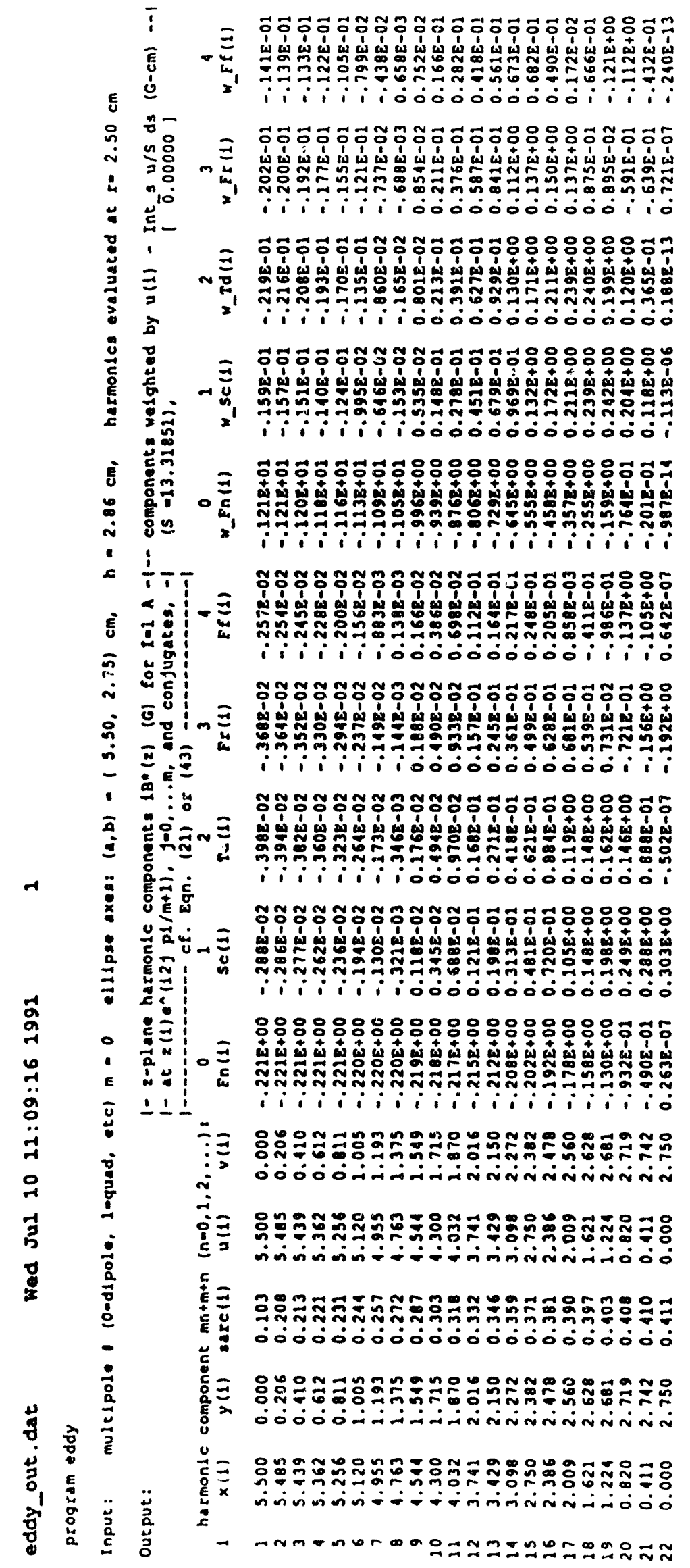

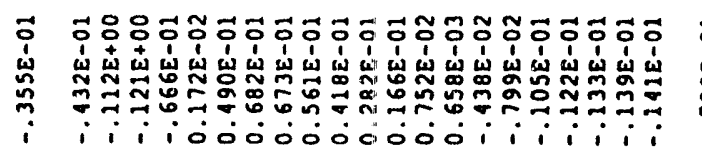

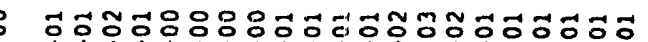

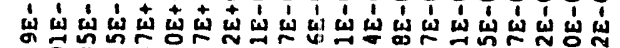

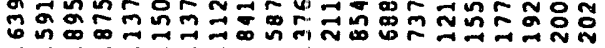

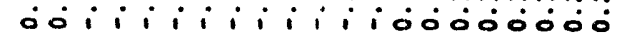

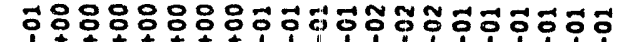

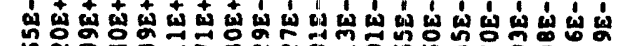

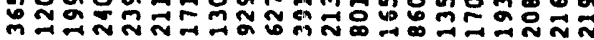

0000000000000ii i i i i

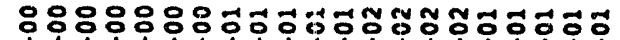

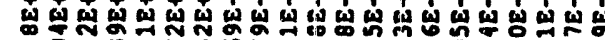

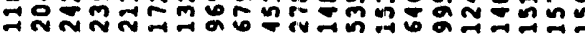

i i i i i i i jo000000

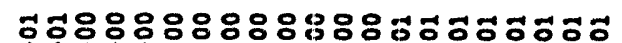

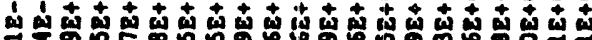

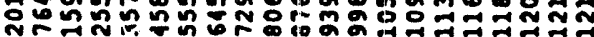

i i i i i i i i i i i i

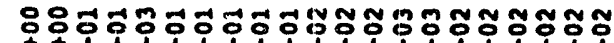

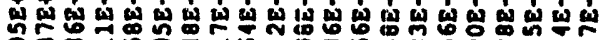

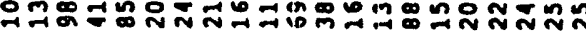
0000ii i i i i i0000000

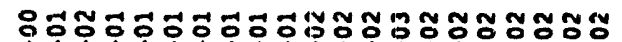

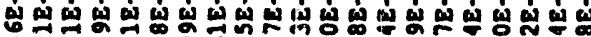

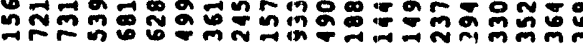

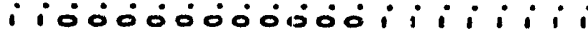

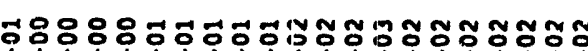

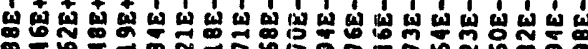

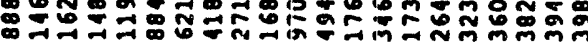
$i i_{i} i_{i} i i_{i} i$ ojoojoo

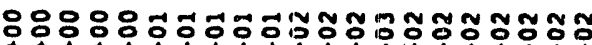

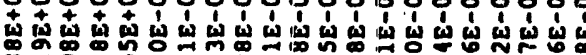

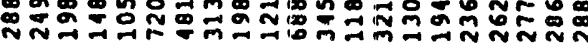

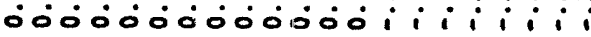

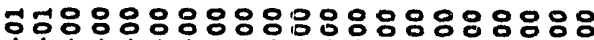

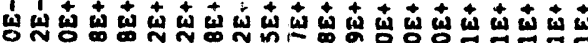

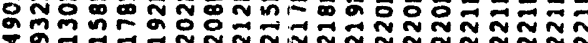
- 000000000000000000

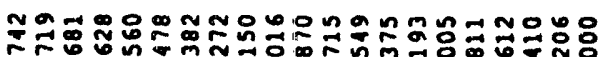

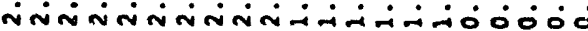

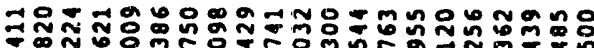

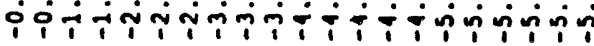

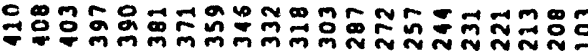

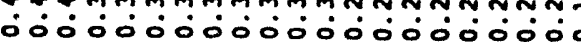

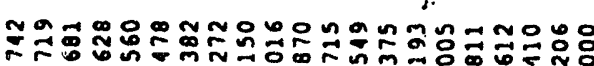

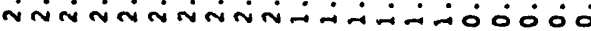

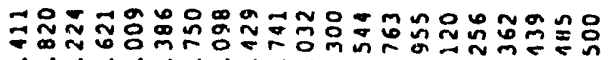

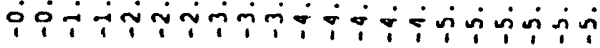

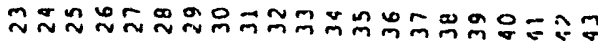




$$
\frac{B_{I_{\text {vac }}}^{a}(z)}{\sigma D \frac{d B_{\text {cail }}}{d t}}=\frac{\mu_{0}}{2 h i} \Sigma_{0} \frac{-1^{n+1} \alpha^{n}}{n !} \int_{s} \operatorname{Re}\left[b_{n}\right] \frac{d I_{s}}{\sigma D \frac{d B_{I_{\text {cail }}}}{d \ell}} d s=\frac{\mu_{0}}{2 h i} \Sigma_{0} \frac{-1^{n+1} \alpha^{n}}{-!} \int_{s} \operatorname{Re}\left[b_{n}\right] x d s
$$

where the $b_{n}$ are as defined in Eqn. (21) with $z_{0} \in s$ and integration is along the vacuum chamber arc in the upper half $z$-plane (which for our elliptical strawman vacuum chamber shape is defined by $s(z)=a_{x} \cos \phi+i b_{y} \sin \phi$, with $a_{x}=5.5 \mathrm{~cm}, b_{y}=2.75 \mathrm{~cm}$, and $0<\phi<\pi$ ). Eqn. (33) is valid only when the vacuum chamber exhibits midplane symmetry.

For $h=2.86 \mathrm{~cm}$, (the LEB dipole magnet half gap), harmonics at $|r|=2.5 \mathrm{~cm}$ as given in closed form by Eqn. (33) are $\frac{i B_{i_{\text {qae }}}(z)}{\sigma D\left(d B_{I_{\text {coil }}} / d t\right)}=-8.65+1.14-0.0709 \frac{G}{A / \mathrm{an}^{2}}$ for the dipole, sextupole, and decapole components, respectively (See Figure 6). These results for an ideal dipole field are virtually identical to those obtained numerically by POISSON for the LEB dipole magnet geometry in the previous section (Figure 5, column 2). The driver program EDDY for evaluation of the harmonic components given by Eqn. (33) for input vacuum chamber dimensions in a background time dependent multipole (dipole for this case) field is found in Appendix $C$.

\subsubsection{Passive Eddy Current Induced Harmonics Suppression}

Compensating eddy current carrying conductor filaments can provide passive nulling of harmonic components. For a filament of uniform cross section at $z_{0}$ and its return at $z_{\infty}$. both parallel to the beam axis, the eddy current density induced in the filament is (cf. Eqn. (11) or (31)):

$$
\begin{gathered}
\frac{j_{z_{0}}}{d B_{I_{\text {coil }}} / d t}=\frac{\sigma}{B_{1}}\left(-A\left(I_{1}, z_{0}\right)+\frac{1}{a_{\text {tot }}} \int_{a} A\left(I_{1}, z\right) d a\right)= \\
\frac{\sigma}{B_{1}}\left(-A\left(I_{1}, z_{0}\right)+\frac{A\left(I_{1}, z_{0}\right) a_{z_{0}}+A\left(I_{1}, z_{\infty}\right) a_{z_{\infty}}}{a_{z_{0}}+a_{z_{\infty}}}\right)=\frac{\sigma\left(A\left(I_{1}, z_{\infty}\right)-A\left(I_{1}, z_{0}\right)\right)}{2 B_{1}}
\end{gathered}
$$

since conductor area $a_{z_{\infty}}=a_{z_{0}}$. For a dipole magnet $\frac{\left(A\left(I_{1}, x_{\infty}\right)-A\left(I_{1}, z_{0}\right)\right)}{B_{1}}=-\left(x_{\infty}-x_{0}\right)$. The compensating current flowing in the conductor at $z_{0}$ is then:

$$
\frac{I_{2_{0}}}{d B_{I_{\text {coil }}} / d t}=\frac{-\sigma a_{20}\left(x_{\infty}-x_{0}\right)}{2} \equiv \frac{-\sigma a_{20} \Delta x}{2}
$$

The return current location $z_{\infty}$ can be posicioned where it does not contribute to the near on-axis field.

Using a pair of compensating coils at $z_{0}$ and $z_{0}^{\prime \prime}$ of magnitude $+I_{z_{0}}$ given by Eqn. (35) in Eqn. (21) (with their returns at $z_{\infty}$ ), we have for the magnitude of the field correction:

$$
\frac{B_{I_{z_{0}}}(z)}{d B_{I_{\text {coil }}} / d t}=\frac{-\mu_{0} \sigma a_{z_{0}} \Delta x}{4 h i} \Sigma_{0} \frac{-1^{n+1} \alpha^{n}}{n !} R_{c}\left[b_{n}\right]
$$


where the $b_{n}=b_{n}\left(\alpha_{0}\left(z_{0}\right)\right)$. As an example, say we wish to null both the vacuum chamber eddy current induced dipole and sextupole terms with four compensating conductors attached somewhere to the vacuum chamber (with their returns located far from the region of interest). From Figure 6 (or Figure 5), the ratio of the $B_{I_{\text {vac }}}$ dipole to sextupole terms is $\frac{-8.65}{1.14} \simeq-7.5$. From Figure 6 , filaments on the vacuum chamber at $(x, y) \simeq$ $( \pm 3.41, \pm 2.16)$ provides this same dipole to sextupole ratio $\left(\frac{-.210}{.0280} \simeq-7.5\right)$. A maximum current magnitude in each of four conductors $I_{x_{0}}=I_{x_{0}^{0}}=-I_{-z_{0}}=-I_{-x_{0}^{0}}=47.5 \mathrm{~A}$ $=\left(\frac{1}{2}\right)\left(\frac{20.0 \mathrm{G}}{0.210 \mathrm{Amp}}\right)$. corresponding to $\left.\frac{d B_{t_{\text {ceil }}}}{d t}\right|_{\max }=367,566 \mathrm{G} / \mathrm{s}$ (see Fig. 5 , condition \#1), would null both the dipole and sextupole vacuum chamber eddy current induced components. Two conductors of maximum current $I_{2_{0}}=I_{x_{0}^{*}}=95 \mathrm{~A}=\left(\frac{20.0}{0.210}\right)$ would also null both these harmonics, but would create a quadrupole component. From Eqn. (35), a copper wire conductor with conductivity $1 / \sigma=1.74 \mu \Omega \mathrm{cm}(\sim 27$ times that of the vacuum chamber), of cross section $a_{20}=0.00750 \mathrm{~cm}^{2}$ (diameter $=0.0977 \mathrm{~cm}$ ) would be required for return filaments $z_{\infty}$ positioned in a dipole field such that $\Delta x=6 \mathrm{~cm}$. In practice, the copper filament cross section should be several times larger and tuned with a series resistor. Then the exact location of the return filaments is not important; they can be positioned anywhere beyond the corner of the tip, away from the gap so that they do not directly influence the good field region.

The eddy current conducting copper filaments cannot occupy the exact same physical location as the vacuum chamber; code EDDY (Appendix C) can be used to calculate the dipole/sextupole suppression ratio for an arbitrarily positioned conductor.

\subsubsection{Dipole Vacuum Chamber Shaping}

The strawman dipole vacuum chamber design results in maximum eddy current induced dipole and sextupole components of $\sim 50 \cdot 10^{-4}$ and $\sim 7 \cdot 10^{-4} B_{I_{\text {coil }}}$ at $|\mathrm{r}|=2.5 \mathrm{~cm}$, respectively (see Figure 5, condition \#2). While the uniform dipole component does not jegrade the field quality, tracking with the quadrupole is adversely affected. The sextupole component is $\sim$ an order of magnitude larger than the field quality specification allows. The cleanest solution is to null both these components, as outlined above. First, however it is desirable to alter the vacuum chamber shape so as to suppress the initial (uncorrected) harmonics level itself as much as possible.

For any given vacuum chamber shape, code EDDY allows rapid evaluation of vacuum chamber eddy current induced harmonics as well as determination of compensating passive harmonics suppression coil locations without resorting to lengthy POISSON code runs.

Figure 7 shows candidate dipole vacuum chamber shapes. For these candidate shapes, in Figure 8 are tabulated eddy-current induced dipole/sextupole ratio, wall thickness, maximum eddy current induced field, and maximum current required in each of four compensating coils along with their approximate locations along the vacuum chamber so as to null both sextupole and dipole vacuum chamber eddy current induced harmonics. (See Appendix C for 'dipole vacuum chamber options' EDDY output files.)

Option $D$ reduces both the eddy current induced (dipole) harmonic and the maximum required corrector coil current by a factor of $\sim 4.6$ over that of the strawman design $A$. The sextupole harmonic is also a factor of $\sim 3.5$ smaller. Part of the improvement is due 
* Inner rull aperture: $52 \mathrm{~mm} \times 107 \mathrm{~mm}$, elliptlcal

- Wall thickness: $3.0 \mathrm{~mm}$

A

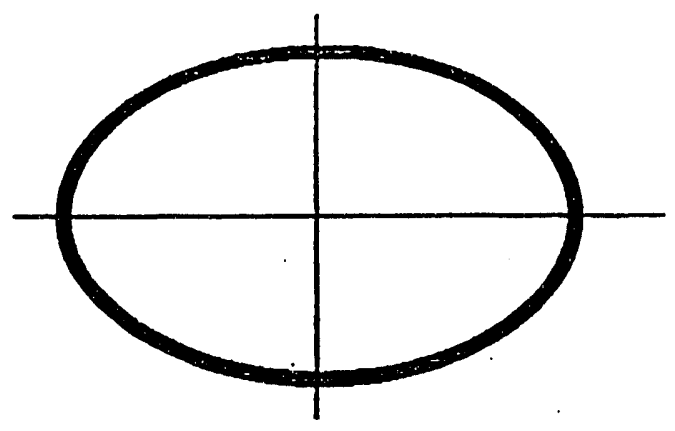

C

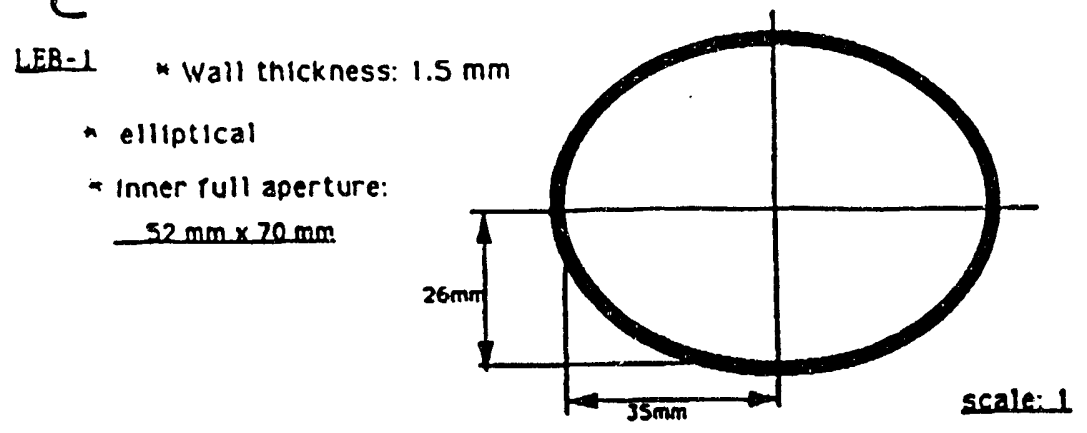

* Inner rull aperture: $52 \mathrm{~mm} \times 82 \mathrm{~mm}$, elliptical

* Wall thickness: $1.5 \mathrm{~mm}$

B

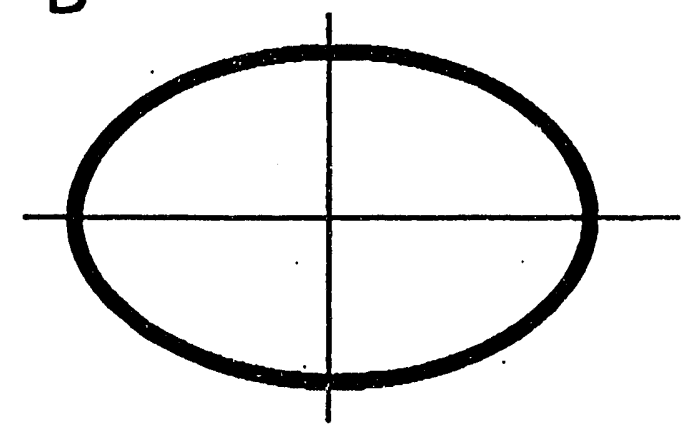

D

LEB-2 * Wall thickness: $1.5 \mathrm{~mm}$

* stretched circle

* Inner rull aperture:

$52 \mathrm{~mm} \times 62 \mathrm{~mm}$

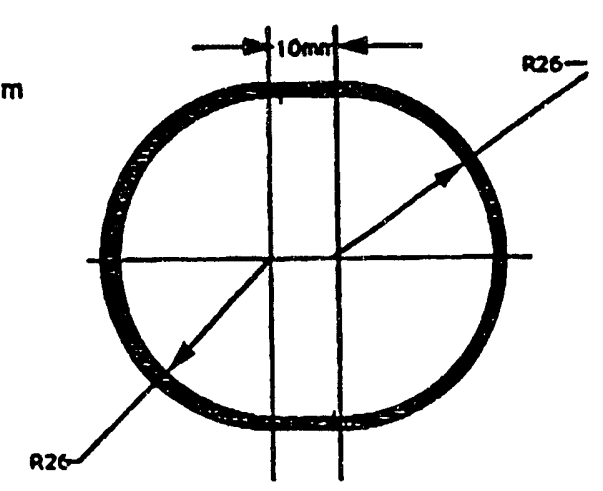

Figure 7. Candidate LEB dipole vacuum chamber shapes.

\begin{tabular}{|c|c|c|c|c|c|c|c|c|c|}
\hline \# & label & $a_{x}$ & $b_{y}$ & $\begin{array}{l}\text { horiz. } \\
\text { stretch } \\
\text { length }\end{array}$ & $\begin{array}{l}\text { Dip/Sext } \\
\text { frm vac ch } \\
\text { eddy curs. }\end{array}$ & $\underset{\mathrm{mm}}{\mathrm{D}}$ & $\begin{array}{c}i B_{I_{\text {sac }}^{*}}^{*} \text { (Dip) } \\
\text { max } B_{I_{\text {cail }}} \\
\text { (cond. } \# 2 \text { ) }\end{array}$ & $\begin{array}{c}I_{\max }(\mathrm{A}) \\
\text { to correct } \\
\text { (cond. \#1) }\end{array}$ & $\begin{array}{l}\text { corrector } \\
\text { location } \\
\text { on vac. ch. }\end{array}$ \\
\hline A & & 5.5 & & - & -7.59 & 3.0 & 0.487 & 47 & 16) \\
\hline B & 1 & & & - & & & & 15 & \\
\hline C & leb \#1 & 3.575 & 2. & & & 1. & 0.122 & 11. & 1.72) \\
\hline D & leb \#2 & 2.675 & .675 & 1.0 & -5.70 & 1.5 & 0.106 & 10.3 & $(2.62,1.62)$ \\
\hline
\end{tabular}

Figure 8. Eddy current induced harmonics (at $r=2.5 \mathrm{~cm}$ ) and required maximum compensating coil currents and location for the candidate vacuum chamber shapes of

Figure 7 and excitation parameters in Figure 5 

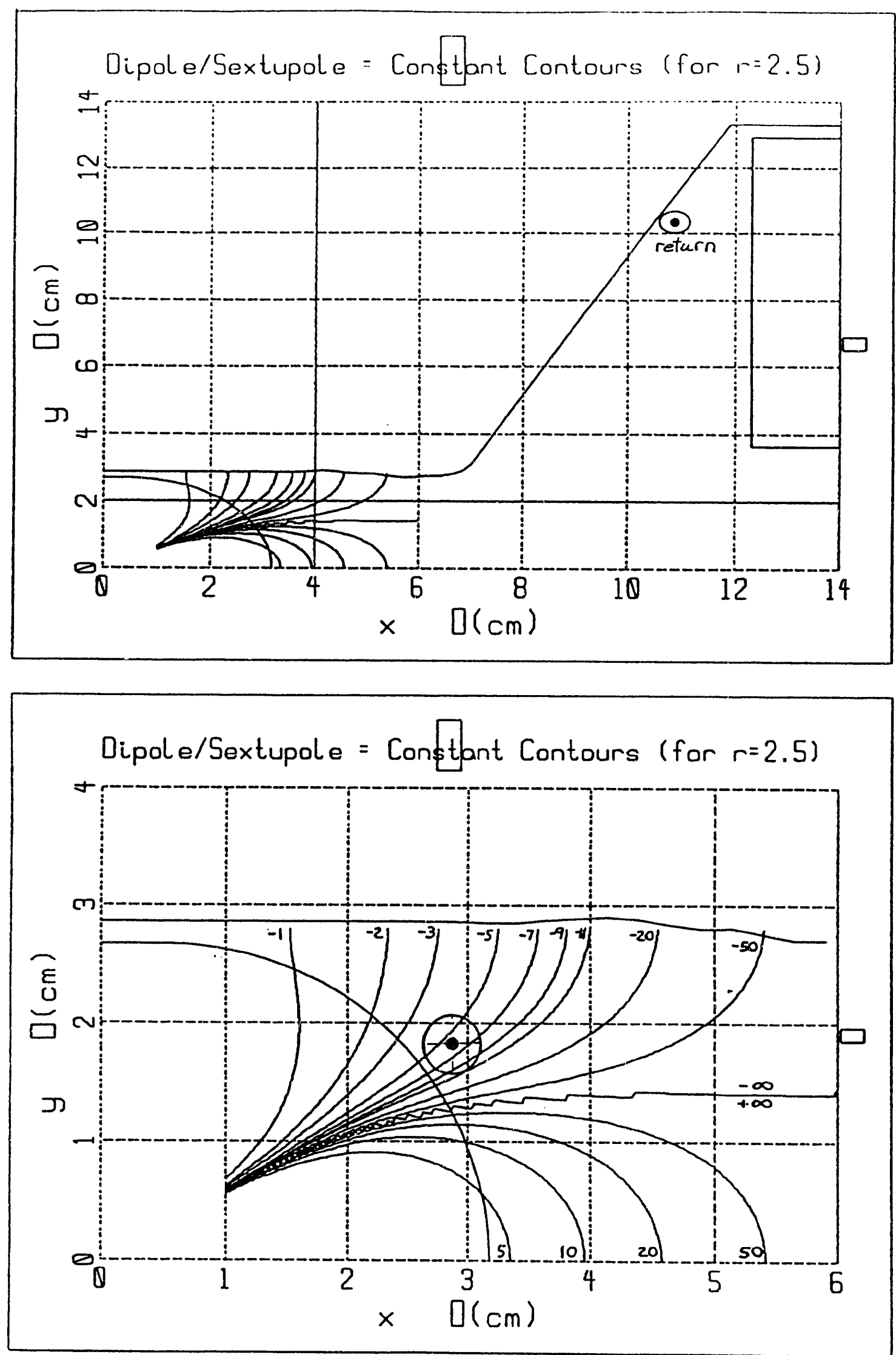

Figure 9. Contours of constant dipole/sextupole at $r=2.5 \mathrm{~cm}$ in a dipole with half gap $h=2.86 \mathrm{~cm}$. 
to the decreased thickness and part to the improved contour of the vacuum chamber.

For the conductor conductivity above and $\Delta x=6 \mathrm{~cm}$ in a dipole field, the required corrector coil diameter for option $D$ is $d=0.0455 \mathrm{~cm},\left(a_{z_{0}}=0.00163 \mathrm{~cm}^{2}\right)$.

Let's make the copper conductor with $d=0.1 \mathrm{~cm}$ (a cross-sectional safety margin of $\sim 5$ ) and assume an insulating/protection sheath of outer diameter $0.5 \mathrm{~cm}$. Figure 9 can be used to position the corrector coil as shown near the vacuum chamber along the dipole/sextupole $=-5.70$ contour at the location $(2.86,1.84)$. (Alternatively, see 'coil positioning' EDDY output files in Appendix $C$ for exact positioning). The coil returns should be placed greater than $6 \mathrm{~cm}$ away horizontally around the tip corner, as shown.

Precise placement of the compensating coils (as well as tuning of the series resistor) is necessary because we are nulling two harmonic components. Figures 8 and 9 show that a $1 \mathrm{~mm}$ error in compensating coil position can lead to as much as a $0.01 \% B_{c_{\text {cail }}}$ step in the sextupole component at $r=2.5 \mathrm{~cm}$. Ideally, coil positioning tolerances should be such that the remaining sextupole field dwarves the field quality spec; a tolerance $\sim 0.25 \mathrm{~mm}$ (10 mils) achieves this. Alternatively, field quality errors due to coil mispositioning can be reduced at the expense of dipole/quadrople magnet tracking by tuning the correction coil resistor so as to completely null the sextupole term at the expense of partial dipole nulling.

The decapole component for option $D$ is $0.5 \cdot 10^{-4} B_{I_{\text {coil }}}$ at $|r|=2.5 \mathrm{~cm}$; it's within the strawman field quality specification, but not 'in the noise'.

\section{SSC Low Energy Booster Quadrupole Magnet Design}

The strawman geometry for the SSC low energy booster quadrupole magnet is shown in Figure 10. The near on-axis field has components due to both the magnet coil excitation and the eddy current excitation in the vacuum chamber as given by Eqns. (25) and (26).

The strawman field quality specification for these magnets are that $\frac{\Delta B}{B} \leq 10^{-3}$ at $|z|=2.0 \mathrm{~cm}$. (later changed to $\frac{\Delta B}{B} \leq 10^{-3}$ at $|z|=4.25 \mathrm{~cm}$.) The excitation is to produce a field strength that cycles between $\sim 1.6$ and $\sim 16 \mathrm{~T} / \mathrm{m}$. (Recall that the quadrupole and dipole are powered together and thus the exact quadrupole field strength is determined by the current required to meet the dipole extraction field strength). The quadrupole aperture is $5 \mathrm{~cm}$.

Maintaining the quadrupole quality of the first term of Eqn. (25) consists of designing the pole so as to avoid magnetic saturation and shaping the tip so as to compensate for the finite shape of the pole. The LEB quadrupole magnet design and harmonics tabulations for various magnet coil excitations are discussed elsewhere ${ }^{[3,4]}$. Specifications are summarized in Appendix $A$.

Maintaining the quadrupole quality of the second term of Eqn. (25) consists of either shaping the vacuum chamber or adding compensating eddy current carrying conductor filaments so as to null harmonic components due to the eddy current distribution in the vacuum chamber conductor. Eddy currents in the vacuum chamber are insensitive to magnet design changes improving the quality of near on-axis field, thus field quality 


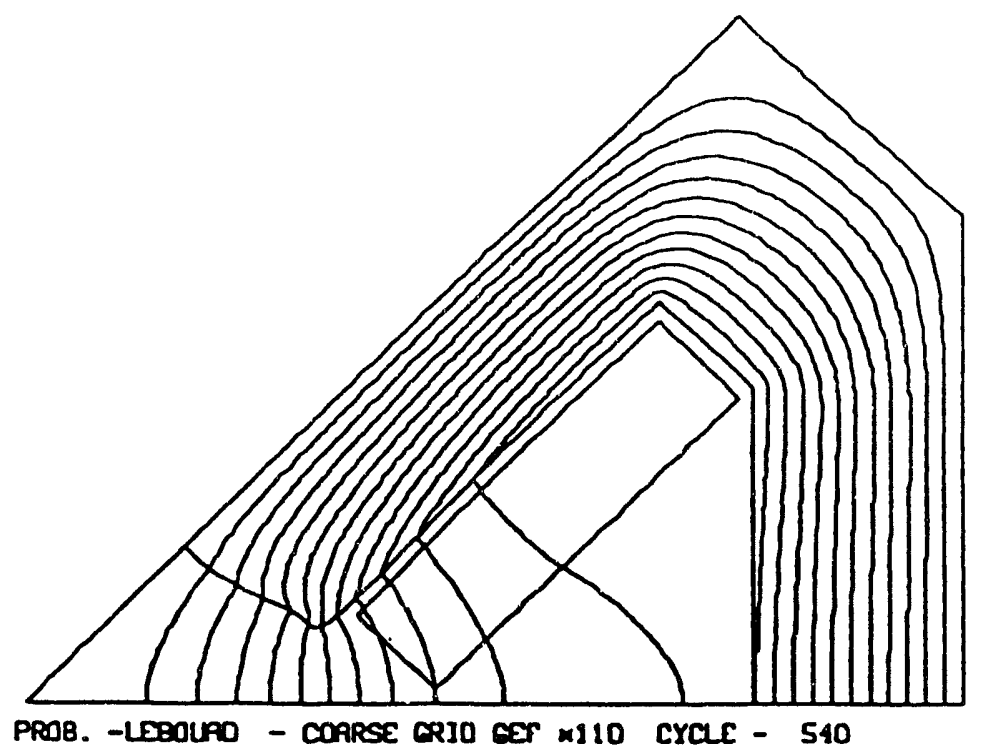

Figure 10. SSC Low Energy Booster quadrupole magnet genmetry.

improvement modifications associated with the two contributions to the quadrupole given by Eqn. (25) may be decoupled.

\subsection{Suppression of Pole Magnet Geometry and Iron Saturation Induced Harmonics}

Harmonics suppression via designing the pole so as to avoid magnetic saturation and shaping the tip so as to compensate for the truncated hyperbolic pole shape is outside the principal thrust of this memo and is discussed elsewhere ${ }^{[3,1]}$.

\subsection{Suppression of Vacuum Chamber Eddy Current Induced Harmonics}

\subsubsection{Mapping Quadrupole Geometry to Dipole Geometry}

To facilitate calculation of eddy current induced harmonics in the region of a time dependent multipole field it is useful to first map the multipole $z$-plane geometry, including the vacuum chamber to a dipole $w$-plane geometry, calculate an analytic variable of interest such as $B^{*}(w)$ in the $w$-plane geometry, and then map back to the original $z$-plane geometry. For the quadrupole geometry, we use the transformation $w \equiv u+i v=z^{2} / r_{p}$, where $r_{p}$ is the quadrupole aperture radius. The elliptical vacuum chamber described by $z=a_{x} \cos \phi+i b_{y} \sin \phi$ is transformed to an ellipse in $w: w=c_{u}+a_{u} \cos \gamma+i b_{v} \sin \gamma$. where $c_{u}=\left(a_{x}^{2}-b_{y}^{2}\right) / 2 r_{p}, a_{u}=\left(a_{x}^{2}+b_{y}^{2}\right) / 2 r_{p}$, and $b_{v}=\left(2 a_{x} b_{y}\right) / 2 r_{p}$.

The quadrupole magnet induced complex magnetic potential

$$
F(w) \equiv A+i V=-w B+c_{2}
$$


from which it follows $B^{*}(w)=i \frac{d F}{d w}=i B(t)$. The transformation back to the $z$-plane gives for $B^{*}(z)$ :

$$
B^{*}(z)=i \frac{d F}{d w} / \frac{d z}{d w}=i B(t) 2 z / r_{p}
$$

\subsubsection{Magnet Excitation}

For the LEB quads $\frac{2 B_{\max }(t)}{r_{p}} \simeq 1.6 \mathrm{kG} / \mathrm{cm}$ and $\frac{2 B_{\min }(t)}{r_{p}} \simeq 0.16 \mathrm{kG} / \mathrm{cm}$. Thus we have

$$
\frac{2 B(t)}{r_{p}}=\frac{2 B_{d c}}{r_{p}}+\frac{2 B_{\Delta}}{r_{p}} \cos \omega t
$$

where $\frac{2 B_{A}}{T_{p}}=0.72 \mathrm{kG} / \mathrm{cm}$ and $\frac{2 B_{d c}}{T_{p}}=0.88 \mathrm{kG} / \mathrm{cm}$.

For this excitation pattern the maximum $\left|\frac{d B / d t}{B}\right|$ that the protons see is at $\omega t \simeq 144.9^{\circ}$ (see Figure 3 or Eqn. (29)). Here $2 B(t) / r_{p}=0.2909 \mathrm{kG} / \mathrm{cm}$ and $\left|\frac{d B / d t}{B}\right|=1.423 \omega$.

\subsubsection{Numerical Adaptation of Vacuum Chamber Eddy Current Effects to POISSON}

The eddy current induced harmonic components for the 'strawman' LEB vacuum chamber geometry (see Figure 8 ) from a POISSON run with current filaments $I_{s ;}$ input according to Eqn. (31), with $\sigma D \frac{d B_{I_{\text {cail }}}}{d t}=1 \mathrm{~A} / \mathrm{cm}^{2}$ and with the $A\left(I_{1}, s_{j}\right), B_{1} \equiv\left|\frac{B^{*}(x) r_{P}}{i 2 z}\right|$ (see Eqn. (37)), and $d s_{j}$ from the quadrupole magnet excitation POISSON run with $I_{\text {coil }}$ set equal to an arbitrary value $I_{1}$, as discussed above Eqn. (11), are tabulated in Figure 11. Sample POISSON files are found in Appendix B. POISSON results for eddy current induced harmonics for the LEB quadrupole magnet are summarized in Figure 12.

5.2.4 Closed Form Analytical Prediction of Eddy Current Induced Harmonics for LEB Quadrupole Magnet

For a quadrupole magnet mapped to dipole geometry according to the $z$-w transformation described above, we have from Eqn. (11) or (31):

$$
I_{v a c}^{\prime}\left(s_{z} \in z, t\right)=\sigma D \frac{d B_{I_{\text {cail }}}}{d t}\left(\frac{u\left(s_{z}\right) B_{1}-R e\left[c_{2}\right]}{B_{1}}-\frac{1}{S} \int_{\text {all } s_{z}} \frac{u\left(s_{z}\right) B_{1}-R e\left[c_{2}\right]}{B_{1}} d s_{z}\right)
$$

where ${ }^{\prime} \equiv \frac{d}{d s_{z}}$ and $s_{z}$ is the arc location in the $z$-plane.

Choosing $c_{2}=0 \Longrightarrow A_{1}(u=0)=0$ and $\int_{s_{z}} \frac{u\left(s_{z}\right) B_{1}-c_{2}}{B_{1}} d s_{z}=\int_{s_{z}} u\left(s_{z}\right) d s_{z} \equiv \hat{u} S$. Thus we have for the current sheet density:

\footnotetext{
${ }^{3}$ The choice of $c_{2}$ is arbitrary; Eqn. (40) results for any choice.
} 
TABLE FOR FIELD COEFFICIENTS

NORMALIZATION RADIUS $=\quad 2.50000$
$\begin{array}{cccc}(B X-I B Y)=I * S U M & N *(A N+I B N) / R *(2 / R) * *(N-1) \\ N & N(A N) / R & N(B N) / R & A B S(N(C N) / R) \\ 2 & 2.0267 E+00 & 0.0000 E+00 & 2.0267 E+00 \\ 4 & -3.9091 E-02 & 0.0000 E+00 & 3.9091 E-02 \\ 6 & -7.7138 E-03 & 0.0000 E+00 & 7.7138 E-03 \\ 8 & -1.9802 E-02 & 0.0000 E+00 & 1.9802 E-02\end{array}$

Figure 11. Vacuum chamber eddy current induced magnecic field harmonics for strawman LEB quadrupole magnet design

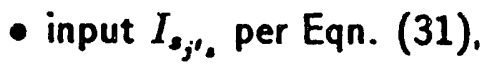

- LEB quadrupole magnet geometry per Figure 10, aperture $r_{p}=5.0 \mathrm{~cm}$

- elliptical vacuum chamber with major half-axes of $5.5 \mathrm{~cm}$ and $2.75 \mathrm{~cm}$

At $|r|=2.5 \mathrm{~cm}$ :

\begin{tabular}{|c|c|c|c|c|c|c|}
\hline $\begin{array}{l}\text { harmonic } \\
\text { component }\end{array}$ & 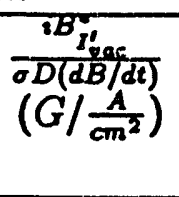 & $\begin{array}{r}\frac{B_{y_{\text {tac }}^{\prime}}^{d B d d t}(\mathrm{~s})}{18}=47 \mu \Omega \mathrm{cm} . \\
D=0.03 \mathrm{~cm}\end{array}$ & $\begin{array}{r}i B_{I t}^{*} \\
\text { at cond } \\
\frac{2}{r_{p}} \sigma D \frac{d B}{d t}\end{array}$ & $\begin{array}{l}\text { (G) } \\
\text { ion \#1: } \\
2.887 \frac{A}{\mathrm{~cm}^{3}}\end{array}$ & $\begin{array}{r}i E \\
\text { at co } \\
\frac{2}{r_{p}} \sigma D \frac{d l}{d}\end{array}$ & $\begin{array}{l}c(G) \\
\text { tion \#2: } \\
=1.660 \frac{\mathrm{A}}{\mathrm{cm}^{3}}\end{array}$ \\
\hline & & & (G) & $\left(\% B_{I_{\text {coil }}}\right)$ & (G) & $\left(\% B_{I_{\text {cail }}}\right)$ \\
\hline $\begin{array}{l}\text { quadrupole: } \\
\text { octupole: } \\
\text { 12-pole: }\end{array}$ & $\begin{array}{r}-2.0267 \\
0.0391 \\
0.0077 \\
\end{array}$ & $\begin{array}{r}-12.94 \cdot 10^{-6} \\
0.2496 \cdot 10^{-6} \\
0.0491 \cdot 10^{-6}\end{array}$ & $\begin{array}{r}-1.463 \\
0.02822 \\
0.07556\end{array}$ & $\begin{array}{r}-.0665 \\
.0013 \\
.0003\end{array}$ & $\begin{array}{r}-0.8411 \\
0.01623 \\
0.00320\end{array}$ & $\begin{array}{r}-.1156 \\
.0022 \\
.0004\end{array}$ \\
\hline
\end{tabular}

where conditions \#1 and $\# 2$ are defined by

- an excitation pattern: $\omega=20 \pi / \mathrm{s}, 2 B_{d c} / r_{p}=.88 \mathrm{kG} / \mathrm{cm}, 2 B_{\Delta} / r_{p}=.72 \mathrm{kG} / \mathrm{cm}$,

- a vacuum chamber conductivity $\sigma, \frac{1}{\sigma}=47 \mu \Omega \mathrm{cm}$ and thickness $D=0.03 \mathrm{~cm}$

\begin{tabular}{|c|c|c|c|c|c|c|}
\hline $\begin{array}{c}\text { condition } \\
\#\end{array}$ & condition & $\overline{\omega t}$ & $\begin{array}{l}\frac{2 B_{I_{\text {coil }}}}{r_{p}} \\
(\mathrm{G} / \mathrm{cm})\end{array}$ & $\begin{array}{l}\frac{2\left(d B_{\left.I_{\text {coil }} / d t\right)}^{r_{p}}\right.}{(G / \mathrm{s}-\mathrm{cm})} \\
(\mathrm{G})\end{array}$ & $\begin{array}{l}\frac{d B_{l_{\text {coil }} / d t}}{B_{I_{\text {coil }}}} \\
\text { (s) }\end{array}$ & $\begin{array}{c}\frac{2 \sigma D}{r_{p}} \frac{d B_{l_{\text {coil }}}}{d t}\left(\frac{A}{c \mathrm{~m}^{3}}\right) \\
\text { at } D=0.03 \mathrm{~cm} \text {. } \\
\frac{1}{\sigma}=47 \mu \Omega \mathrm{cm}\end{array}$ \\
\hline 1 & $B_{I_{\text {vac }}^{\prime}}$ maximized & $90^{\circ}$ & 880.0 & 45,239 & 51.4 & 0.2887 \\
\hline 2 & $\frac{B_{t_{\text {vac }}^{\prime}}}{B_{\text {col }}}$ maximized & $144.9^{\circ}$ & 290.9 & 26,015 & 89.4 & 0.1660 \\
\hline
\end{tabular}

Figure 12. LEB quadrupole magnet eddy current induced harmonics from POISSON 
Figure 13. LEB quadrupole magnet eddy current induced harmonics (analytical)

E

$\boldsymbol{H}$

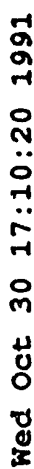

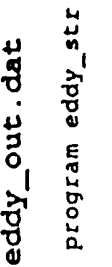

$\bar{i}$ 戛

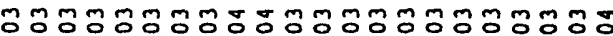

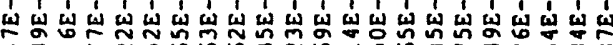

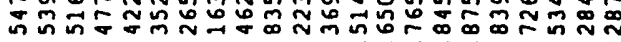

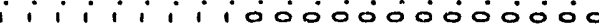

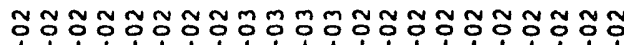

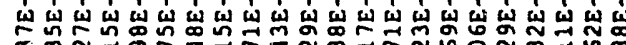

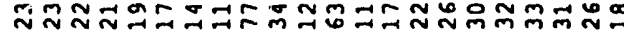

ii i i i i i i iojojojodoojo

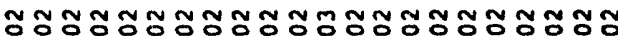

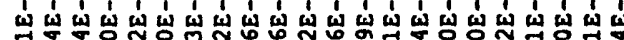

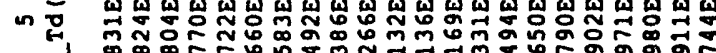

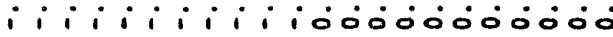

$\Xi$

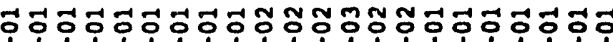

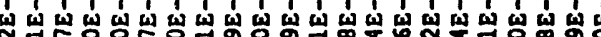

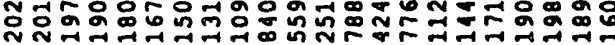

$i_{i} i_{i} i_{i} i_{i} i_{i}$ oojojojoo

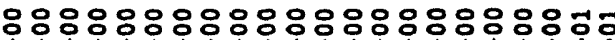

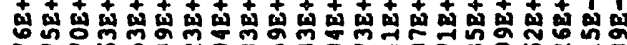

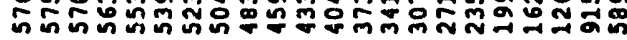

i i i i i i i i i i i i i i i i i

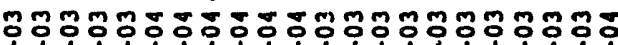

由

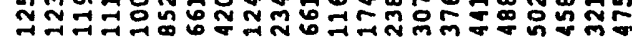

ii i i i i ioojojoojo0000

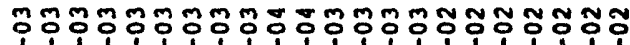

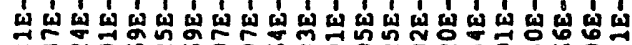

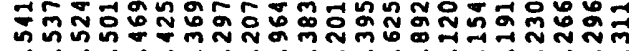

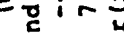

त的

要亩

:

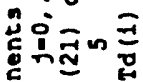

高家

8 E

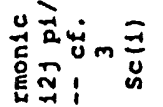

1 i i i i i i i000000000000

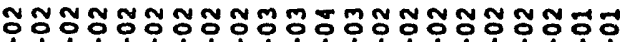

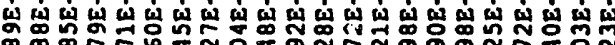

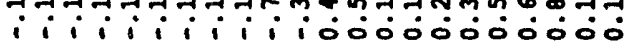

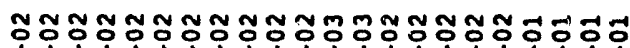

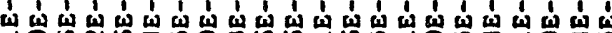

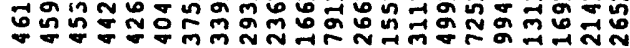

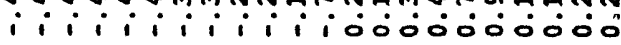

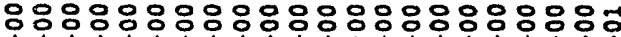

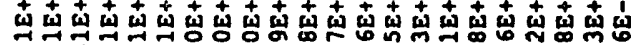

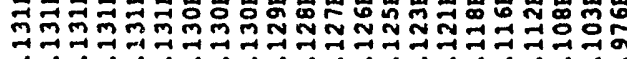

$i_{i} i_{i} i_{i} i i_{i} i_{i} i_{i} i_{i} i_{i}$

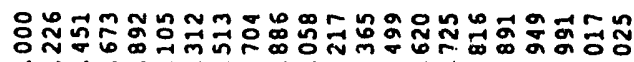

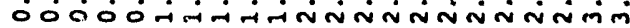

$\stackrel{5}{1}$

茫

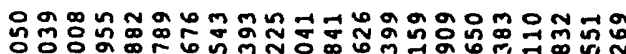

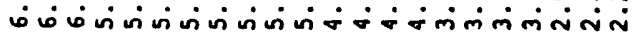

c

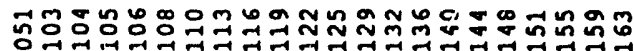
00000000000000000000

要

要三

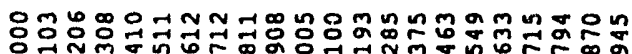

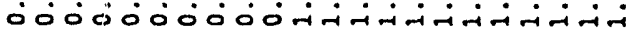

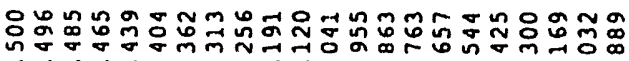

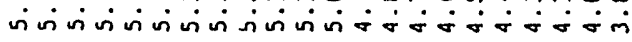

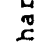

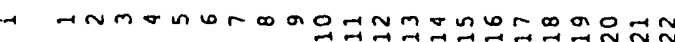

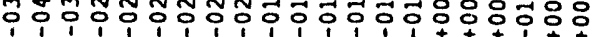

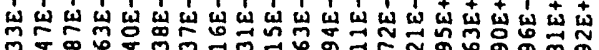

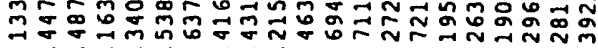

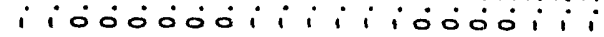

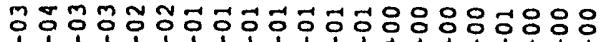

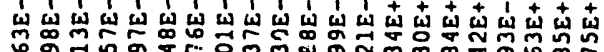

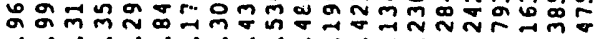

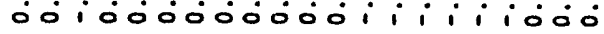

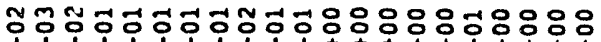

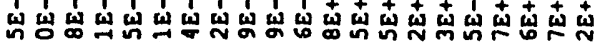

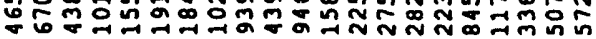
óii i i iojoojojoji i i

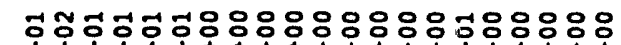

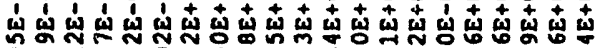

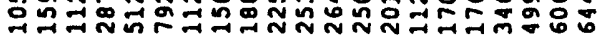
ooi i i i i i i i i i ioojojo

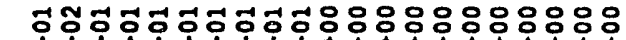

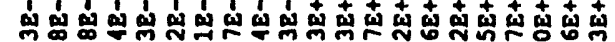

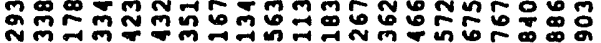

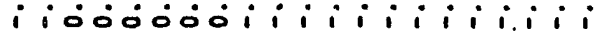

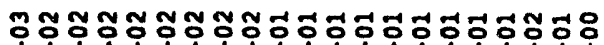
ผ

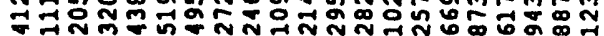
i i i i i i iojojooi i i iojo

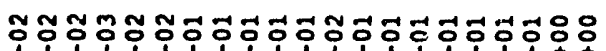

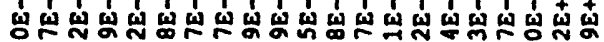

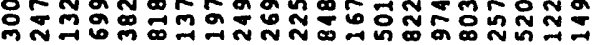
oooi i i i i i idoododoi i

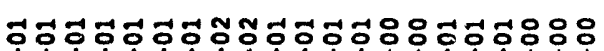

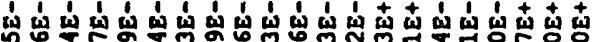
ᄀㅜㄱ óo0ojodi i i i i i i ioojo

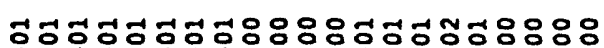

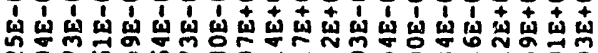

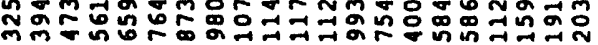
oododododoojodili i i

|

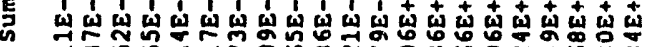
ลิ๊ i i i i i i iojojojojojoo

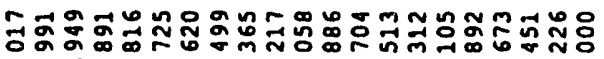

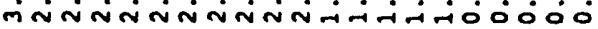

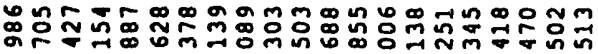

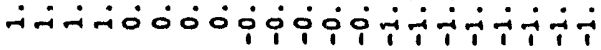

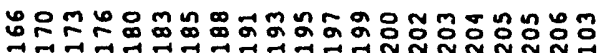

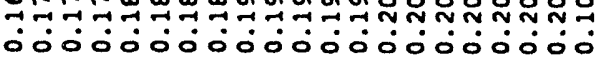
咠员

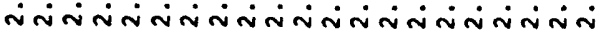

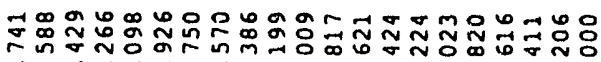

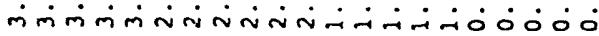
ฟ o

$$
\text { . }
$$




$$
\frac{I_{\mathrm{uac}}^{\prime}\left(s_{z} \in z, t\right)}{\sigma D(d B / d t)}=u-\frac{1}{S} \int_{a l l, s_{z}} u\left(s_{z}\right) d s_{z} \equiv u-\hat{u}
$$

where integration is along the entire vacuum chamber arc. Using this value for $I_{\text {vac }}^{\prime}\left(s_{z}, t\right)$ in the expression for eddy current induced harmonics Eqn. (21) yields

$$
\frac{B_{I_{\text {vac }}}^{*}(w)}{\sigma D \frac{d B_{I_{\text {coil }}}}{d t}}=\frac{\mu_{0}}{i 2 r_{p}} \Sigma_{0} \frac{-1^{n+1} \alpha^{n}}{n !} \int_{s_{x}} \operatorname{Re}\left[b_{n}\right] \frac{d I_{s}}{\sigma D \frac{d B_{I_{\text {coil }}}}{d t}} d s_{z}=\frac{\mu_{0}}{i 2 r_{p}} \Sigma_{0} \frac{-1^{n+1} \alpha^{n}}{n !} \int_{z_{z}} \operatorname{Re}\left[b_{n}\right](u-\hat{u}) d s_{z}
$$

where the $b_{n}$ are as defined in Eqn. (21), $\alpha \equiv \frac{\pi w}{2 r_{p}}, \alpha_{0} \equiv \frac{\bar{\pi} w_{0}}{2 r_{p}}, u=u\left(s_{z}\right)$, and integration in Eqn. (41) is along the vacuum chamber arc in the the first quadrant in the $z$-plane (equivalently, upper half $w$-plane).

Finally, making the transformation back to the $z$-plane, $B_{z}^{*}=B_{w}^{*} / \frac{d z}{d w}=\frac{2 z}{r_{p}} B_{w}^{*}$ and we have for $B_{z}^{*}$ :

$$
\frac{B_{I_{\text {vac }}}^{*}(z)}{\sigma D \frac{d_{I_{\text {coil }}}}{d t}}=\frac{\mu_{0}}{i r_{p}} \Sigma_{n=0} \frac{-1^{n+1}\left(\frac{x}{2}\right)^{n}\left(\frac{z}{r_{p}}\right)^{2 n+1}}{n !} \int_{z z} \operatorname{Re}\left[b_{n}\right](u-\hat{u}) d s_{z}
$$

where the $b_{n}=f\left(\alpha_{0}\right), u=u(w(z)), \alpha_{0}=\alpha_{0}\left(u_{i}\left(z_{0}\right)\right), \hat{u} \equiv \frac{1}{s} \int_{a_{z}} u\left(s_{z}\right) d s_{z}, d s_{z} \in z$, and integration is along the vacuum chamber arc in the first quadrant in the $z$-plane. Eqns. (41) and (42) are valid only for a quadrupole vacuum chamber exhibiting symmetry about both $y=0$ and $x=0$.

For $r_{p}=5.0 \mathrm{~cm}$. (the LEB quadrupole magnet aperture), harmonics for the 'strawman' LEB vacuum chamber geometry (see Figure 8 ) at $|r|=2.5 \mathrm{~cm}$ as given in closed form by Eqn. (42) are $\frac{i B_{i_{\text {sac }}}(z)}{\sigma D\left(d B_{I_{\text {coil }}} / d t\right)}=-2.08+0.0347+0.0136 \frac{G}{A / \mathrm{cm}^{2}}$ for the quadrupole, octupole, and 12-pole components, respectively (See Figure 13). These results for an ideal quadrupole field are virtually identical to those obtained numerically by POISSON for the LEB quadrupole magnet geometry in the previous section (Figure 12, column 2). The driver program EDDY for evaluation of the harmonic components given by Eqn. (42) for input vacuum chamber dimensions in a background time dependent multipole (quadrupole for this case) field is found in Appendix $C$.

\subsubsection{Quadrupole Vacuum Chamber Shaping}

The strawman quadrupole vacuum chamber design $(A)$ results in maximum eddy current induced quadrupole and octupole components of $\sim 11.5 \cdot 10^{-4}$ and $\sim 0.2 \cdot 10^{-4} B_{I_{\text {coill }} \text {. }}$. respectively (see Figure 12, condition \#2). While the quadrupole component does not degrade the field quality, tracking with the dipole magnet is adversely affected; the goal is for dipole-quadrupole tracking to within $1 \cdot 10^{-3}$. The octupole and 12-pole components are well within the field quality specification. If an altered vacuum chamber shape can 


\begin{tabular}{|c|c|c|c|c|c|c|c|c|}
\hline$\#$ & label & $a_{x}$ & $b_{y}$ & $\begin{array}{l}\text { horiz. } \\
\text { stretch } \\
\text { length }\end{array}$ & $\begin{array}{l}\text { Quad/Oct } \\
\text { frm vac ch } \\
\text { eddy curs. }\end{array}$ & $\begin{array}{l}\text { Quad/12-pole } \\
\text { frm vac ch } \\
\text { eddy curs. }\end{array}$ & $\begin{array}{c}D \\
(\mathrm{~mm})\end{array}$ & $\begin{array}{l}i B_{I_{\text {vac }}^{*}}^{*} \text { (Quad) } \\
\left(\max \% B_{I_{\text {coil }}}\right. \\
\text { i.e., cond. \#2) }\end{array}$ \\
\hline $\begin{array}{l}A \\
B \\
C \\
D\end{array}$ & $\begin{array}{l}\text { straw } \\
1^{\text {st }} \text { mod } \\
\text { leb \#1 } \\
\text { leb \#2 }\end{array}$ & $\begin{array}{l}5.5 \\
4.2 \\
3.575 \\
2.675 \\
\end{array}$ & $\begin{array}{l}2.75 \\
2.7 \\
2.675 \\
2.675 \\
\end{array}$ & $\begin{array}{c}- \\
- \\
- \\
1.0\end{array}$ & $\begin{array}{l}-60 \\
-26 \\
-27 \\
-32 \\
\end{array}$ & $\begin{array}{l}-153 \\
-178 \\
-236 \\
+67.5 \\
\end{array}$ & $\begin{array}{l}3.0 \\
1.5 \\
1.5 \\
1.5 \\
\end{array}$ & $\begin{array}{l}0.119 \\
0.040 \\
0.033 \\
0.030 \\
\end{array}$ \\
\hline $\begin{array}{l}E \\
F \\
G\end{array}$ & $\begin{array}{l}\text { circ } 4.5 \\
\text { circ } 4.0 \\
\text { circ } 3.5\end{array}$ & $\begin{array}{l}4.575 \\
4.075 \\
3.575\end{array}$ & $\begin{array}{l}4.575 \\
4.075 \\
3.575\end{array}$ & $\begin{array}{l}- \\
- \\
-\end{array}$ & $\begin{array}{l}\infty \\
\infty \\
\infty\end{array}$ & $\begin{array}{l}-89 \\
-122 \\
-183\end{array}$ & $\begin{array}{l}1.5 \\
1.5 \\
1.5\end{array}$ & $\begin{array}{l}0.065 \\
0.050 \\
0.039\end{array}$ \\
\hline
\end{tabular}

Figure 14. Maximum eddy current induced harmonics (at $r=2.5 \mathrm{~cm}$ ) for various candidate quadrupole vacuum chamber shapes and the excitation pattern of Figure 12

sufficiently suppress the quadrupole so that tracking with the dipole is not greatly affected, we can do without corre tion coils for the quadrupole magnet altogether.

Figure 14 shows tabulated maximum eddy current induced harmonics for various candidate quadrupole vacuum chamber shapes.

Any of the design options except $A$, which has a thicker vacuum chamber wall, will give maximum yuadrupole harmonics of less than the strawman design specification $1 \cdot 10^{-3}$ $B_{I_{\text {coil }}}$ at $|r|=2.0 \mathrm{~cm}$. In particular, option $F$, featuring a $4.0 \mathrm{~cm}$ ID circular vacuum chamber gives a maximum quadrupole harmonic of $0.5 \cdot 10^{-3} B_{I_{\text {coil }}}$ at $|r|=2.5 \mathrm{~cm}$. At $|r|=4.0 \mathrm{~cm}$, however, option $F$ gives a maximum quadrupole harmonic of $0.8 \cdot 10^{-3}$ of $B_{I_{\text {coil }}}$. All higher order harmonics are negligible at this radius, thus quadrupole field quality is not significantly degraded by vacuum chamber eddy currents.

The magnitude of the vacuum chamber eddy current induced quadrupole term for these vacuum chamber shapes may be sufficiently low depending on the final, dipole-quadrupole tracking design specification and tolerance to risk; correction coils may not be needed in the quadrupole magnet.

\section{Generalization of Analytical Expressions for an $m^{\text {th }}$ Order Multipole of 'Symmetrical' Vacuum Chamber Shape}

6.1 Generalization of Analytical Expressions for Vacuum Chamber Eddy Current Induced Harmonics in an $m^{\text {th }}$ Order Multipole of 'Symmetrical' Vacuum Chamber Shape

Let $m$ be the order of the multipole magnet ( $m=0,1,2, \ldots$ for dipole, quadrupole, sextupole, etc.) Mapping the multipole magnet in the $z$-plane onto a dipole geometry in $w$ according to the prescription $w \equiv u+i v=z^{m+1} / r_{p}^{m}$, where $r_{p}$ is the multipole 
aperture $\left(r_{p} \longrightarrow h\right.$ for a dipole magnet), determining the value of $B^{*}(w)$ in $w$, and then mapping back to $z$, yields for $m+1$ pairs of current filaments of magnitude $+I$ at $z_{0} e^{\frac{i 2 j \pi}{m+1}}$ and $\left(z_{0} e^{\frac{i 2 j \pi}{m+1}}\right) *$ where $j=0, \ldots m$ :

$$
i B^{*}(z)=i B_{x}+B_{y}=\frac{(m+1) \mu_{0} I}{2 r_{p}} \Sigma_{n=0} \frac{-1^{n+1}\left(\frac{\pi}{2}\right)^{n}\left(\frac{z}{r_{p}}\right)^{n m+n+m}}{n !} \operatorname{Re}\left[b_{n}\right]
$$

where the $b_{n}=f\left(\alpha_{0}\right)$ and $\alpha_{0} \equiv \frac{\pi u_{n}}{2 r_{p}}$.

The harmonic components induced by eddy currents in vacuum chambers restricted to be symmetric ${ }^{4}$ with respect to the lines in the $z$-plane $\theta=\frac{j \pi}{(m+1)}, j=0,1, \ldots 2 m+1$, are given by:

$$
i B_{I_{\text {tec }}}^{*}(z)=\frac{(m+1) \mu_{0}}{2 r_{p}} \Sigma_{n=0} \frac{-1^{n+1}\left(\frac{\pi}{2}\right)^{n}\left(\frac{z}{r_{p}}\right)^{n m+n+m}}{n !} \int_{s_{z}} \operatorname{Re}\left[b_{n}\right] I_{v a c}^{\prime}\left(s_{z}\right) d s_{z}
$$

where the kernel $I_{\text {vac }}^{\prime}\left(s_{z}\right)$ is giver, by

$$
\frac{I_{v a c}^{\prime}\left(s_{z} \in z, t\right)}{\sigma D(d B / d t)}=u-\frac{1}{S} \int_{a l l} u\left(s_{z}\right) d s_{z}
$$

where the $b_{n}=f\left(\alpha_{0}\right)$ are $w$-plane variables and the integration in Eqn. (44) is along the vacuum chamber arc: $0 \leq \theta\left(s_{z}(z)\right) \leq \pi /(m+1)$ (along the entire arc for Eqn. (45)).

The code EDDY of Appendix C evaluates the expressions given by Eqns. (43) and (44) for a multipole of arbitrary order with a specified 'symmetric' (as defined above) vacuum chamber shape. Note that Eqns. (21) and (33) are just the dipole specific versions of Eqns. (43) and (44), respectively.

If we have an additional $m+1$ pairs of current filaments of magnitude $-I$ located at $z_{0} e^{\frac{i \pi(2 j+1)}{m+1}}$ and $\left(z_{0} e^{\frac{i m(2 j+1)}{m+1}}\right)^{*}$, where $j=0, \ldots m$, analogous to Eqn. (43) we obtain:

$$
i B^{*}(z)=i B_{x}+B_{y}=\frac{(m+1) \mu_{0} I}{r_{p}} \Sigma_{n=\text { even }} \frac{-\left(\frac{\pi}{2}\right)^{n}\left(\frac{z}{r_{p}}\right)^{n m+n+m}}{n !} \operatorname{Re}\left[b_{n}\right]
$$

Thus, harmonic components induced by a multipole magnet's vacuum chamber which exhibits the additional symmetry about the $z$-plane lines $\theta=\frac{(1+2 j) \pi}{2(m+1)}, j=0, \ldots 2 m+1$ is:

$$
i B_{I_{\text {vac }}}^{*}(z)=\frac{(m+1) \mu_{0}}{r_{p}} \Sigma_{n=\text { even }} \frac{-\left(\frac{\pi}{2}\right)^{n}\left(\frac{z}{r_{p}}\right)^{n m+n+m}}{n !} \int_{s_{z}} \operatorname{Re}\left[b_{n}\right] I_{v a c}^{\prime}\left(s_{z}\right) d s_{z}
$$

${ }^{1}$ Note that a non-circular, elliptical vacuum chamber in a sextupole magnet does not exhibit this symmetry, and thus Egn. (44) does not apply. This interesting case is treated separately in Appendix D. 
where the kernel $I_{v a c}^{\prime}\left(s_{z}\right)$ is given by Eqn. (45). Note that Eqn. (24) is just the dipole specific version of Eqn. (47).

\subsection{Generalization of Passive Eddy Current Induced Harmonics Suppres-} sion in an $m^{\text {th }}$ Order Multipole

Compensating eddy current carrying conductor filaments can provide passive nulling of harmonic components in any $m^{\text {th }}$ order multipole. Mapping to dipole (w) geometry, for a filament of uniform cross section $z_{0}$ and its return at $z_{\infty}$, both parallel to the beam axis, the eddy current density induc is in the filament is (cf. Eqns. (11) and (31)):

$$
\begin{gathered}
\frac{j_{z_{0}}}{d B_{I_{\text {coil }}} / d t}=\frac{\sigma}{B_{1}}\left(-A\left(I_{1}, w_{0}\right)+\frac{1}{a_{\text {tot }}} \int_{\alpha} A\left(I_{1}, w\right) d a\right)= \\
\frac{\sigma}{B_{1}}\left(-A\left(I_{1}, w_{0}\right)+\frac{A\left(I_{1}, w_{0}\right) a_{z_{0}}+A\left(I_{1}, w_{\infty}\right) a_{z_{\infty}}}{a_{z_{0}}+a_{z_{\infty}}}\right)=\frac{\sigma\left(A\left(I_{1}, w_{\infty}\right)-A\left(I_{1}, w_{0}\right)\right)}{2 B_{1}}
\end{gathered}
$$

since $a_{x_{\infty}}=a_{x_{0}}$. In the mapped dipole geometry, $\frac{\left(A\left(I_{1}, w_{\infty}\right)-A\left(I_{1}, u_{0}\right)\right)}{B_{1}}=-\left(u_{\infty}-u_{0}\right)$. where $B_{1} \equiv\left|\frac{B^{*}(z) r_{p}^{m}}{i(m+1) z^{m}}\right|$. The compensating current flowing in the conductor at $w_{0}$ is then:

$$
\frac{I_{z_{0}}}{d B_{I_{\text {coil }}} / d t}=\frac{-\sigma a_{z_{0}}\left(u_{\infty}-u_{0}\right)}{2}=\frac{-\sigma a_{z_{0}} \Delta u}{2}
$$

The return current location $w_{\infty}$ can be positioned where it does not contribute to the near on-axis field.

Figure 15 summarizes the eddy current induced harmonics and suppression discussion of this section.

Using the magnitude of compensating current $I_{z_{0}}$ given by Eqn. (49) in Eqn. (4j), the magnitude of the field correction in an $m^{\text {th }}$ order multipole with $m+1$ pairs of current filaments of magnitude $+I$ at $z_{0} e^{\frac{i 2 j \pi}{m+1}}$ and $\left(z_{0} e^{\frac{i 2 j \pi}{m+2}}\right.$ ) , where $j=0, \ldots m$ (and with their returns at $z_{\infty}$ ), is

$$
\frac{B_{I_{x_{0}}}^{*}(z)}{d B_{I_{\text {coil }}} / d t}=\frac{-(m+1) \mu_{0} \sigma a_{z 0} \Delta u}{4 r_{p} i} \Sigma_{n=0} \frac{-1^{n+1}\left(\frac{\pi}{2}\right)^{n}\left(\frac{z}{r_{p}}\right)^{n m+m+n}}{n !} \operatorname{Re}\left[b_{n}\right]
$$

where the $b_{n}=b_{n}\left(\alpha_{0} \equiv \frac{\pi w_{0}}{2 r_{p}}\right)$ are $w$-plane variables.

One can null both the vacuum chamber eddy current induced fundamental and next allowed harmonic term with $m+1$ pairs of compensating conductors attached somewhere to the vacuum chamber at the appropriate locations and with their returns located far from the region of interest. (See Figure 15a,c,e.)

For the case of additional vacuum chamber symmetry discussed above Eqn. (47), using the value of compensating current $I_{20}$ given by Eqn. (49) in Eqn. (46), the magnitude 
Figure 15. Summary of eddy current induced harmonics and passive suppression for multipole rnagnets with various generic vacuum chamber shapes

allowed harmonics (POISSON notation) DIPOLE: $m=0$, QUAD $m=1$, ctc. for design rotatable by $\theta^{\circ}=360 \% m^{\prime}$ : where $m^{\prime}=m+1, n^{\prime} \equiv n+1$

\begin{tabular}{c|ccccc|ccccc|}
$m^{\prime}$ & $\left.\begin{array}{c}\text { cur. change sqn. } \\
n^{\prime}=m^{\prime}(2\end{array} M_{-1}\right) / 2$ & \multicolumn{4}{c|}{$\begin{array}{c}\text { cur. samie sign } \\
n^{\prime}=m^{\prime} M\end{array}$} \\
\hline 1 & & & & & & 1 & 2 & 3 & 4 & 5 \\
2 & 1 & 3 & 5 & 7 & 9 & 2 & 4 & 6 & 8 & 10 \\
3 & & & & & & 6 & 9 & 12 & 15 \\
4 & 2 & 6 & 10 & 14 & 18 & 4 & 8 & 12 & 16 & 20 \\
5 & & & & & & 10 & 15 & 20 & 25 \\
6 & 3 & 9 & 15 & 21 & 27 & 6 & 12 & 18 & 24 & 30
\end{tabular}

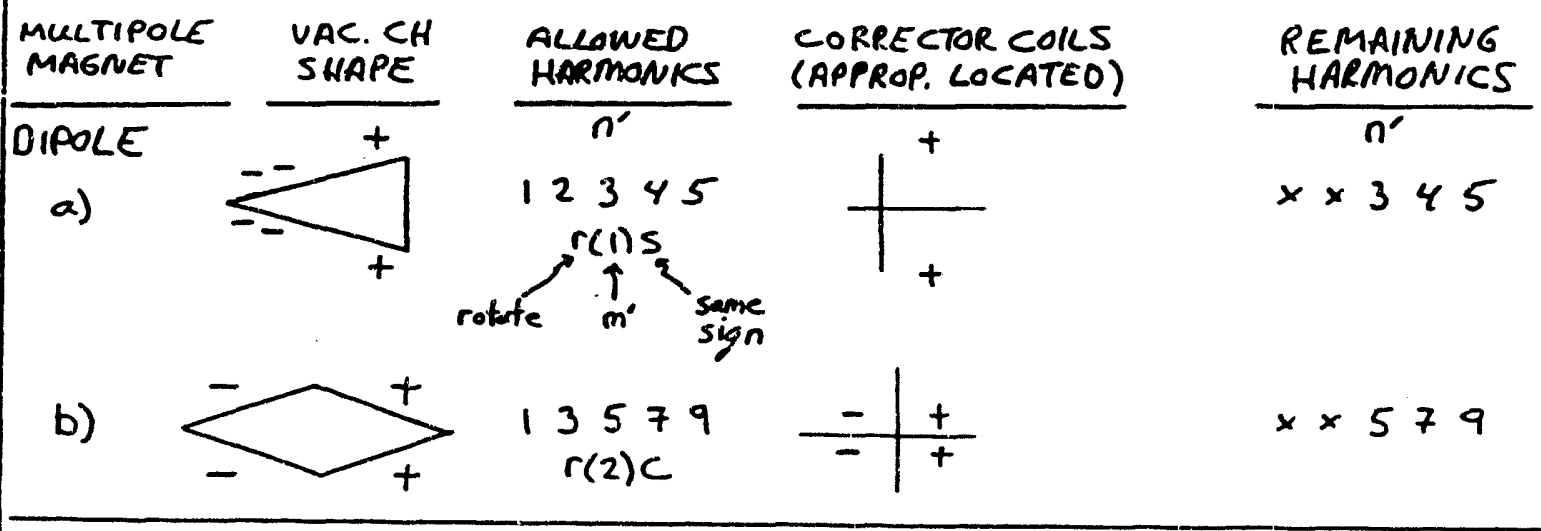

QUAD.

c)

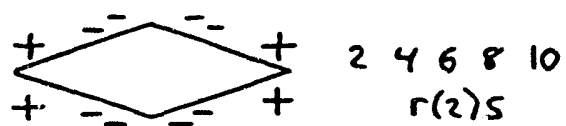

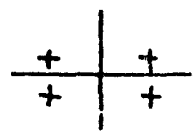
$\times \times 686$ $r(2) 5$ $M=1,2,3,4 \ldots$

$$
\text { d) }
$$

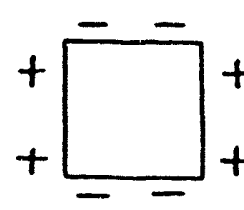

26101418 $r(4) c$

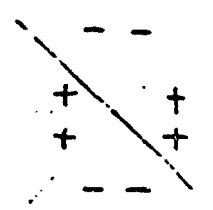

SEXT.

e)

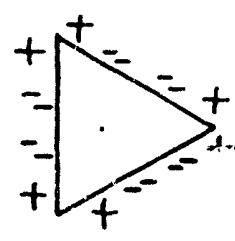

3691215

$r(3) s$

$$
+t^{t}
$$

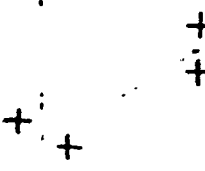

$+$

$\times \times 91215$

f)

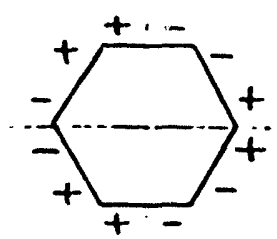

$\begin{array}{ll}39152127 & +{ }^{+}- \\ r(6)< & =+ \\ & ++_{+}+\end{array}$

g) - $_{+^{+}++++_{+}^{+}+-}^{+-}$

$3579=+$
$r(2) c$

$\times \times 579$ 
of the field correction in an $m^{\text {th }}$ order multipole with $m+1$ pairs of current filaments of magnitude $+I$ at $z_{0} e^{\frac{i 2 j \pi}{m+1}}$ and $\left(z_{0} e^{\frac{i 2 j \pi}{m+1}}\right)^{*}$ and $m+1$ pairs of current filaments of magnitude $-I$ at $z_{0} e^{\frac{i(2 j+1) \pi}{m+1}}$ and $\left(z_{0} e^{\frac{i(2 j+1) \pi}{m+1}}\right)^{*}$, where $j=0, \ldots m$ is

$$
\frac{B_{I_{z_{0}}}^{*}(z)}{d B_{I_{\text {coil }}} / d t}=\frac{-(m+1) \mu_{0} \sigma a_{z_{0}} \Delta u}{2 r_{p} i} \Sigma_{n=\text { even }} \frac{-1\left(\frac{\pi}{2}\right)^{n}\left(\frac{z}{r_{p}}\right)^{n m+m+n}}{n !} R e\left[b_{n}\right]
$$

where the $b_{n}=b_{n}\left(\alpha_{0} \equiv \frac{\pi w_{0}}{2 r_{p}}\right)$ are $w$-plane variables.

One can null both the vacuum chamber eddy current induced fundamental and next allowed harmonic term with $2(m+1)$ pairs of compensating conductors attached to the vacuum chamber at the appropropriate locations, and with their returns located far from the region of interest. (See Figure 15b,d,f.)

\subsection{Vacuum Chamber Eddy Current Induced Harmonics in the LEB Sex- tupole Magnets}

The strawman field quality specification for these magnets are that $\frac{\Delta B}{B} \leq 10^{-3}$ at $|z|=2.0$ $\mathrm{cm}$, (later changed to $\frac{\Delta B}{B} \leq 10^{-3}$ at $|z|=4.0 \mathrm{~cm}$.) The excitation is to produce a field strength that cycles between 6 and $60 \mathrm{~T} / \mathrm{m}^{2}$. The sextupole aperture is $5 \mathrm{~cm}$.

\subsubsection{Mapping Sextupole Geometry to Dipole Geometry}

For the sexturole geometry, we use the transformation $w \equiv u+i v=z^{3} / r_{p}^{2}$, where $r_{p}$ is the sextupole aperture radius. The sextupole magnet induced complex magnetic potential

$$
F(w) \equiv A+i V=-w B+c_{2}
$$

from which it follows $B^{*}(w)=i \frac{d F}{d w}=i B(t)$. The transformation back to the $z$-plane gives for $B^{*}(z)$ :

$$
B^{*}(z)=i \frac{d F}{d w} / \frac{d z}{d w}=i B(t) 3 z^{2} / r_{p}^{2}
$$

\subsubsection{Magnet Excitation}

For the LEB sextupoles $\frac{3 B_{\max }(t)}{r_{p}^{2}} \simeq 60 \mathrm{G} / \mathrm{cm}^{2}$ and $\frac{3 B_{\min }(t)}{r_{p}^{2}} \simeq 6 \mathrm{G} / \mathrm{cm}^{2}$. Thus we have

$$
\frac{3 B(t)}{r_{p}^{2}}=\frac{3 B_{d c}}{r_{p}^{2}}+\frac{3 B_{\Delta}}{r_{p}^{2}} \cos \omega t
$$

where $\frac{3 B_{A}}{r_{p}}=27 \mathrm{G} / \mathrm{cm}^{2}$ and $\frac{3 B_{d c}}{r_{p}^{2}}=33 \mathrm{G} / \mathrm{cm}^{2}$. 
- input $I_{s_{j}, \text { }}$ per Eqn. (44).

- ideal sextupole magnet geometry, aperture $r_{p}=5.0 \mathrm{~cm}$

- circular vacuum chamber with radius $4.075 \mathrm{~cm}$

At $|r|=2.5 \mathrm{~cm}$ :

\begin{tabular}{|c|c|c|c|c|c|c|}
\hline $\begin{array}{l}\text { harmonic } \\
\text { component }\end{array}$ & $\frac{B_{d_{\text {vac }}^{\prime}}}{\sigma D(d B / d t)}$ & 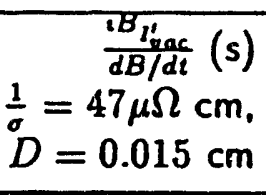 & $\begin{array}{r}i B \\
\text { at cor } \\
\frac{3}{r_{p}^{2}} \sigma D \frac{d B}{d t}\end{array}$ & $\begin{array}{l}I_{\text {vac }}^{\prime}(G) \\
\text { adition \#1: } \\
=.00541 \frac{\mathrm{A}}{\mathrm{cm}^{2}}\end{array}$ & $\begin{array}{r}i L \\
\text { at co } \\
\frac{3}{r_{p}^{2}} \sigma D \frac{d E}{d t}\end{array}$ & $\begin{array}{l}B_{I_{\text {vac }}}(G) \\
\text { ndition \#2: } \\
==.00311 \frac{A}{\mathrm{~cm}^{2}}\end{array}$ \\
\hline & & & (G) & $\left(\%\right.$ of $\left.B_{I_{\text {coil }}}\right)$ & (G) & $\left(\%\right.$ of $\left.B_{I_{\text {coil }}}\right)$ \\
\hline $\begin{array}{l}\text { sextupole: } \\
\text { 18-pole: }\end{array}$ & $\begin{array}{r}-0.794 \\
0.0012 \\
\end{array}$ & $\begin{array}{r}-2.534 \cdot 10^{-6} \\
0.0038 \cdot 10^{-6} \\
\end{array}$ & $\begin{array}{r}-.0358 \\
.00005 \\
\end{array}$ & $\begin{array}{r}-.0130 \\
.00002 \\
\end{array}$ & $\begin{array}{r}-8.411 \\
.0127 \\
\end{array}$ & $\begin{array}{r}-.0226 \\
.00003\end{array}$ \\
\hline
\end{tabular}

where conditions \#1 and \#2 are defined by

- an excitation pattern: $\omega=20 \pi / \mathrm{s}, 3 B_{d c} / r_{p}^{2}=33 \mathrm{G} / \mathrm{cm}^{2}, 3 B_{\Delta} / r_{p}^{2}=27 \mathrm{G} / \mathrm{cm}^{2}$,

- a vacuum chamber conductivity $\sigma, \frac{1}{\sigma}=47 \mu \Omega \mathrm{cm}$ and thickness $D=0.015 \mathrm{~cm}$

\begin{tabular}{|c|c|c|c|c|c|c|}
\hline $\begin{array}{c}\text { condition } \\
\#\end{array}$ & condition & $\omega t$ & $\frac{\frac{3 B_{I_{\text {coil }}}}{r_{\mathrm{p}}^{2}}}{\left(\mathrm{G} / \mathrm{cm}^{2}\right)}$ & $\frac{\frac{3\left(d B_{l_{\text {coil }}} / d t\right)}{r_{p}^{2}}}{\left(G / \mathrm{s}-\mathrm{cm}^{2}\right)}$ & $\frac{d B_{I_{\text {coil }}} / d t}{B_{I_{\text {coil }}}}$ & $\begin{array}{c}\left.\frac{3 \sigma D}{r_{p}^{2}} \frac{d B_{I_{\text {coil }}}\left(\frac{A}{d t}\right)}{\mathrm{cm}^{\mathrm{s}}}\right) \\
\text { at } D=.015 \mathrm{~cm}, \\
\frac{1}{\sigma}=47 \mu \Omega \mathrm{cm}\end{array}$ \\
\hline 1 & $B_{\text {Itac }}$ maximized & $90^{\circ}$ & 33.00 & 1696 & 51.4 & .00541 \\
\hline 2 & $\frac{B_{\text {trac }}^{\prime}}{B_{\text {ex }}}$ maximized & $144.9^{\circ}$ & 10.91 & 975 & 89.4 & .00311 \\
\hline
\end{tabular}

Figure 16. Ideal sextupole magnet eddy current induced harmonics (analytical)

For this excitation pattern the maximum $\left|\frac{d B / d t}{B}\right|$ that the protons see is at $\omega t \simeq 144.9^{\circ}$ (see Figure 3 or Eqn. (29)). Here $3 B(t) / r_{p}^{2}=10.91 \mathrm{G} / \mathrm{cm}^{2}$ and $\left|\frac{d B / d t}{B}\right|=1.423 \omega$.

\subsubsection{Closed Form Analytical Prediction of Eddy Current Induced Harmonics for LEB Sextupole Magnet}

For a vacuum chamber in a sextupole magnet field that is symmetric with respect to the lines in the $z$-plane $\theta=\frac{j \pi}{3}, j=0,1, \ldots 5$, (as is the case for a circular vacuum chamber) the eddy current induced harmonics are given by Eqn. (44) with $m=2$.

For $r_{p}=5.0 \mathrm{~cm}$. (the LEB sextupole magnet aperture), harmonics for a circular 'strawman' LEB sextupole vacuum chamber of radius $r=4.075 \mathrm{~cm}$ and thickness $D=$ $0.015 \mathrm{~mm}$, at $|r|=2.5 \mathrm{~cm}$ as given in closed form by Eqn. (44) are $\frac{i B_{i_{v a c}}(z)}{\sigma D\left(d B_{I_{\text {coil }}} / \mathrm{dt}\right)}=$ $-0.794+0.0012 \frac{G}{A / \mathrm{cm}^{2}}$ for the sextupole and 18-pole components, respectively (See Figure 16. column 2). The driver program EDDY for evaluation of the harmonic components 
given by Eqn. (44) for input vacuum chamber dimensions in a background time dependent multipole (sextupole for this case) field is found in Appendix C.

The strawman sextupole vacuum chamber design results in maximum eddy current induced sextupole and 18-pole components of $\sim 2.3: 10^{-4}$ and $0.003 \cdot 10^{-4} B_{I_{\text {coil }}}$ at $r=2.5 \mathrm{~cm}$, respectively (See Figure 16, condition \#2). Eddy current induced harmonics do not significantly degrade the sextupole field quality; passive correction coils are not needed on the LEB sextupole magnets.

\section{Acknowledgements}

We are happy to acknowledge the helpful insight of Gerry Fischer into various issues related to the work described herein. The discussion of magnet excitation patterns (Section 4.2.1) and their effect on eddy current percentage contributions follows his original observations.

\section{Literature Cited}

1. Reference Manual for the Poisson/Superfish Group of Codes, LA-UR-87-126, Los Alamos National Laboratory, Los Alamos, NM, 1987.

2. Lee, S.Y., A Multipole Expansion for the Field of Vacuum Chamber Eddy Currents, Brookhavem National Laboratory Internal Report AD/AP/T-12.

3. Schlueter, R., LEB Dipole and Quadrupole Magnetics Design Summary, \#1, \#2, \#3. \#4, April-July 1991.

4. Thiagarajan, et.al., Design of Main Dipoles and Quadrupoles for the SSC Low Energy Booster, SSC Report, Dec. 1991.

5. Fischer, G.E., personnal communication. 


\section{Appendix A: Suppression of Harmonics due to Magnet Geometry and Iron Saturation}




\section{Appendix A: Suppression of Harmonics due to Magnet Geometry and Iron Saturation}

See the series of memos ${ }^{[3]}$ entitled LEB Dipole and Quadrupole Magnetics Design Summary. \#1, 2, 3, 4, 4/91-7/91. Final magnet designs yield:

- a dipole magnet with

- a good field region with $\Delta B / B \leq 1 \cdot 10^{-4}$ within the ellipse $z \leq 3.25 \cos \theta+$ $i 2.5 \sin \theta$ at injection, where $B \simeq 1300 \mathrm{G}$,

- a good field region with $\Delta B / B \leq 1 \cdot 10^{-4}$ within the ellipse $z \leq 1.40 \cos \theta+$ il.40 $\sin \theta$ at extraction, where $B=13000 \mathrm{G}$

- a quadrupole magnet with

- a good field region with $\triangle B / B \leq 1 \cdot 10^{-3}$ within the sllipse $z \leq 4.25 \cos \theta+$ $i 4.25 \sin \theta$ at injection, where $d B / d z \simeq 1600 \mathrm{G} / \mathrm{cm}$,

- a good field region with $\triangle B / B \leq 1 \cdot 10^{-3}$ within the ellipse $z \leq 4.00 \cos \theta+$ $i 4.00 \sin \theta$ at extraction, where $d B / d z=16000 \mathrm{G} / \mathrm{cm}$,

- The dipole and quadrupole magnets track to within $1 \cdot 10^{-3}$ over the entire range of operation, injection to extraction. 


\section{Appendix B: POISSON Files for Eddy Current Induced Harmonics of SSC LEB Magnet Designs}

1. LEB001: LEB dipole magnet excitation

2. LEBV001: LEB dipole magnet vacuum chamber eddy current excitation

3. LEBQUAD: $L E B$ quadrupole magnet excitation

4. LEBQUADv: LEB quadrupole magnet vacuum chamber eddy current excitation 


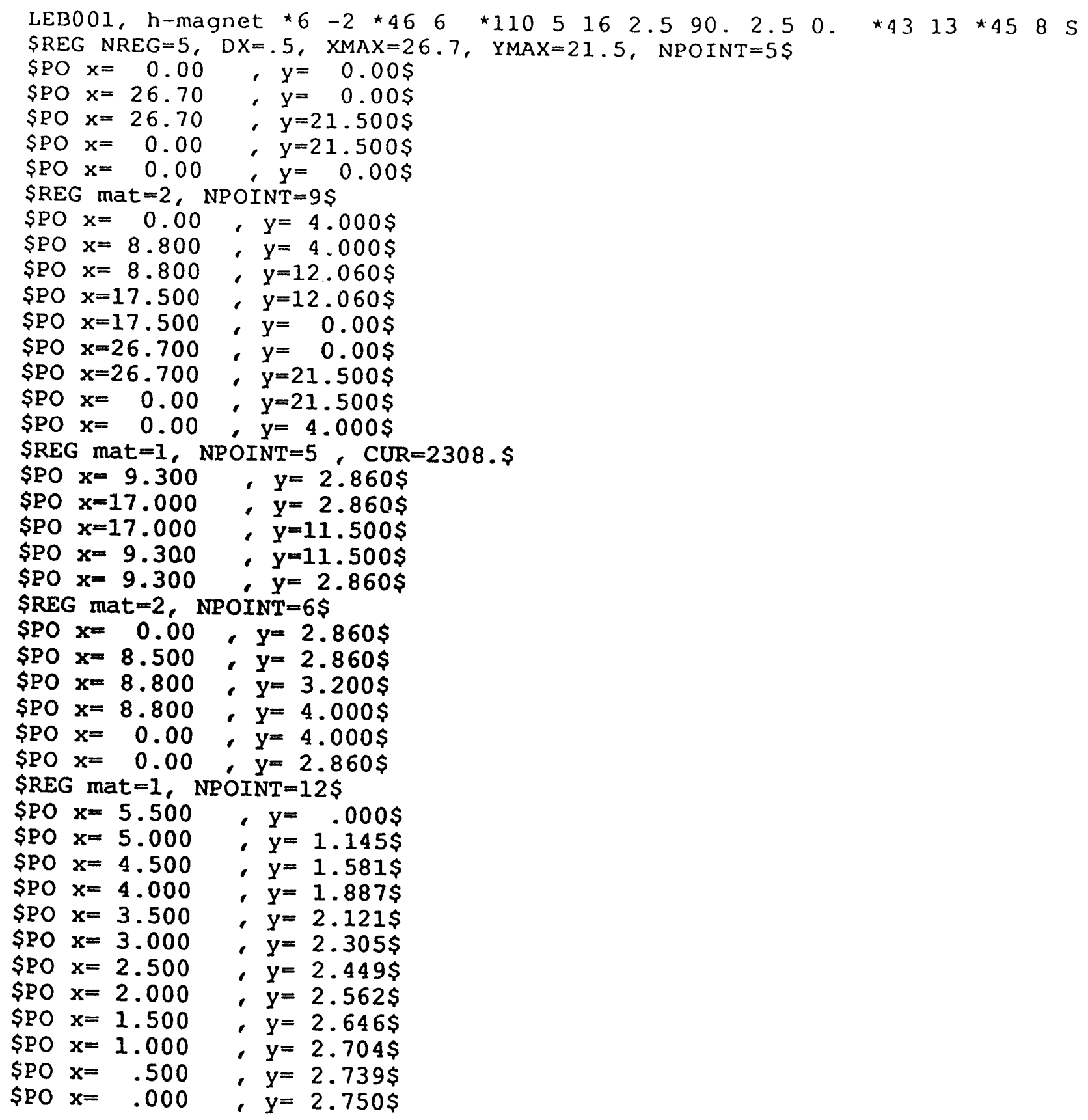




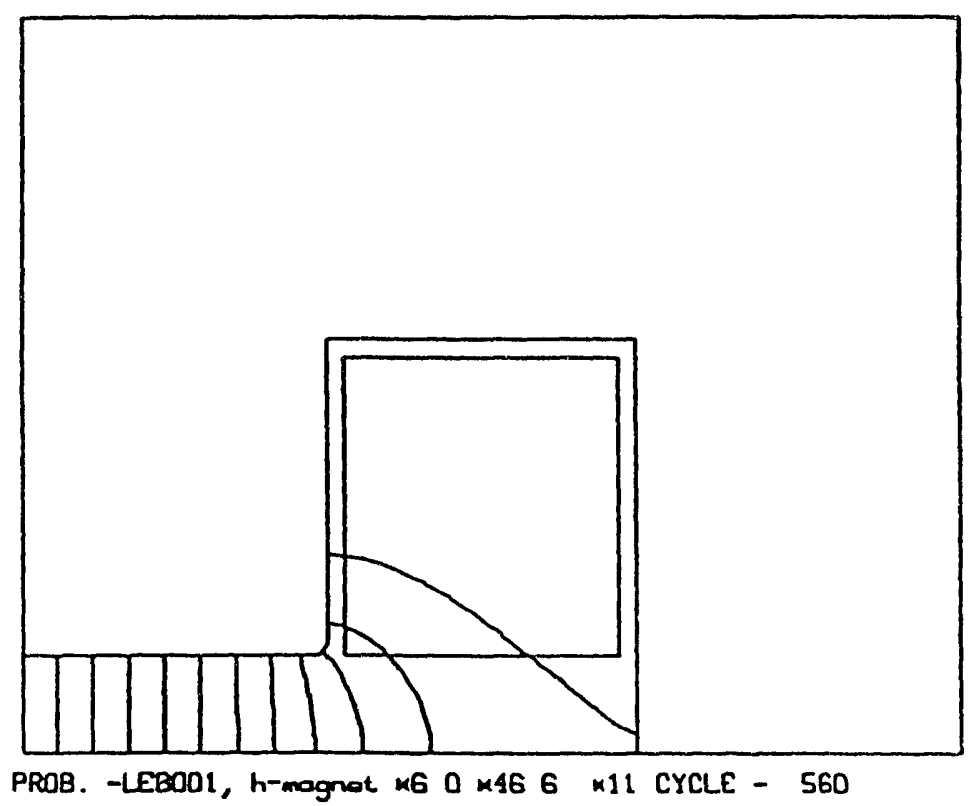


LEAST SQUARES EDIT OF PROBLEM , CYCLE 560 ' $H$ ' MAg SyMMETRY TYPE call. $\frac{-A_{i} \Delta s_{i}}{B_{1}}$ clongvac.ch́. STORED ENERGY $=1.3849 E+01$ JOULES / METER OR RADIAN $X J F A C T=1.000000$

$B_{1}=-1014 G$

\begin{tabular}{|c|c|c|c|c|c|c|c|}
\hline $\mathrm{K}$ & $\mathrm{L}$ & A (VECTOR) & $x$ & $Y$ & BX (GAUSS) & BY (GAUSS) & BT (GAUSS) \\
\hline 1 & 1 & $0.000000 \mathrm{E}+00$ & 0.00000 & 0.00000 & 0.000 & -1014.049 & 1014.049 \\
\hline 2 & 1 & $5.108502 E+02$ & 0.50377 & 0.00000 & 0.000 & -1014.042 & 1014.042 \\
\hline 3 & 1 & $1.021692 \mathrm{E}+03$ & 1.00755 & 0.00000 & 0.000 & -1014.016 & 1014.016 \\
\hline 4 & 1 & $1.532515 E+03$ & 1.51132 & $0: 00000$ & 0.000 & -1013.966 & 1013.966 \\
\hline 5 & 1 & $2.043304 E+03$ & 2.01509 & 0.00000 & 0.000 & -1013.875 & 1013.875 \\
\hline 6 & 1 & $2.554031 E+03$ & 2.51887 & 0.00000 & 0.000 & -1013.725 & 1013.715 \\
\hline 7 & 1 & $3.064650 \mathrm{E}+03$ & 3.02264 & 0.00000 & 0.000 & -1013.439 & 1013.439 \\
\hline 8 & 1 & $3.575083 E+03$ & 3.52642 & 0.00000 & 0.000 & -1012.959 & 1012.959 \\
\hline 9 & 1 & $4.085192 E+03$ & 4.03019 & 0.00000 & 0.000 & -1012.117 & 1012.117 \\
\hline 10 & 1 & $4.594734 E+03$ & 4.53396 & 0.00000 & 0.000 & -1010.670 & 1010.670 \\
\hline 11 & 1 & $5.103309 E+03$ & 5.03774 & 0.00000 & 0.000 & -1008.177 & 1008.177 \\
\hline 12 & 1 & $5.568549 E+03$ & 5.50000 & 0.00000 & 0.000 & -1004.304 & 1004.304 \\
\hline 13 & 1 & $6.114276 \mathrm{E}+03$ & 6.04528 & 0.00000 & 0.000 & -996.601 & 996.601 \\
\hline 1 & 2 & $0.000000 \mathrm{E}+00$ & 0.00000 & 0.43878 & 0.000 & -1014.055 & 1014.055 \\
\hline 2 & 2 & $3.070387 E+02$ & 0.30278 & 0.44057 & 0.008 & -1014.052 & 1014.052 \\
\hline 3 & 2 & $7.877885 E+02$ & 0.77687 & 0.44290 & 0.022 & -1014.038 & 1014.038 \\
\hline 4 & 2 & $1.293525 E+03$ & 1.27562 & 0.44475 & 0.043 & -1014.005 & 1014.005 \\
\hline 5 & 2 & $1.806382 E+03$ & 1.78140 & 0.44700 & 0.079 & -1013.944 & 1013.944 \\
\hline 6 & 2 & $2.322652 E+03$ & 2.29060 & 0.45066 & 0.140 & -1013.833 & 1013.833 \\
\hline 7 & 2 & $2.839386 E+03$ & 2.80032 & 0.45518 & 0.246 & -1013.641 & 1013.641 \\
\hline 8 & 2 & $3.353274 E+03$ & 3.30737 & -0.45620 & 0.428 & -1013.310 & 1013.310 \\
\hline 9 & 2 & $3.864874 E+03$ & 3.81239 & 0.44626 & 0.734 & -1012.724 & 1012.724 \\
\hline 10 & 2 & $4.381410 E+03$ & 4.32270 & 0.42331 & 1.223 & -1011.663 & 1011.664 \\
\hline 11 & 2 & $4.901304 \mathrm{E}+03$ & 4.83708 & 0.39733 & 2.012 & -1009.770 & 1009.772 \\
\hline 12 & 2 & $5.401667 \mathrm{E}+03$ & 5.33333 & 0.38167 & 3.285 & -1006.579 & 1006.584 \\
\hline 13 & 2 & $5.894869 E+03$ & 5.82443 & 0.39850 & 5.695 & -1001.423 & 1001.439 \\
\hline 1 & 3 & $0.000000 \mathrm{E}+00$ & 0.00000 & 0.87755 & 0.000 & -1011.070 & 1014.070 \\
\hline 2 & 3 & $5.435944 E+02$ & 0.53605 & 0.88117 & 0.025 & -1011.066 & 1014.056 \\
\hline
\end{tabular}


LEBV001, h-magnet $* 6-2 \star 46 \quad 6 \star 1105162.590 .2 .50 . * 4313 \star 458 \star 4913 \mathrm{~S}$ SREG NREG $=5, D X=.5, X M A X=26.7, \quad Y M A X=21.5, N P O I N T=5 \$$

\$PO $x=0.00, y=0.00 \$$

SPO $x=26.70, y=0.00 \$$

\$PO $x=26.70, y=21.500 \$$

\$PO $x=0.00, y=21.500 \$$

\$PO $x=0.00, y=0.00 \$$

\$REG mat $=2$, NPOINT $=9 \$$

\$PO $x=0.00, y=4.000 \$$

\$PO $x=8.800, y=4.000 \$$

\$PO $x=8.800, y=12.060 \$$

\$PO $x=17.500, y=12.060 \$$

\$PO $x=17.500, y=0.00 \$$

\$PO $x=26.700, y=0.00 \$$

\$PO $x=26.700, y=21.500 \$$

\$PO $x=0.00, y=21.500 \$$

\$PO $x=0.00, y=4.000 \$$

\$REG mat $=1$, NPOINT $=5 \$$

\$PO $x=9.300, y=2.860 \$$

\$PO $x=17.000, y=2.860 \$$

\$PO $x=17.000, y=11.500 \$$

\$PO $x=9.300, y=11.500 \$$

\$PO $x=9.300, y=2.860 \$$

\$REG mat $=2$, NPOINT $=6 \$$

\$PO $x=0.00, y=2.860 \$$

\$PO $x=8.500, y=2.860 \$$

\$PO $x=8.800, y=3.200 \$$

\$PO $x=8.800, y=4.000 \$$

\$PO $x=0.00, y=4.000 \$$

\$PO $x=0.00, y=2.860 \$$

SREG mat $=1$, NPOINT $=12 \$$

\$PO $x=5.500, y=.000 \$$

\$PO $x=5.000, y=1.145 \$$

\$PO $x=4.500, y=1.581 \$$

\$PO $x=4.000, y=1.887 \$$

\$PO $x=3.500, y=2.121$ \$

\$PO $x=3.000, \quad y=2.305 \$$

\$PO $x=2.500, y=2.449 \$$

\$PO $x=2.000, y=2.562 \$$

\$PO $x=1.500, y=2.646 \$$

\$PO $x=1.000, y=2.704 \$$

\$PO $x=.500, y=2.739 \$$

\$PO $x=.000, y=2.750 \$$ 


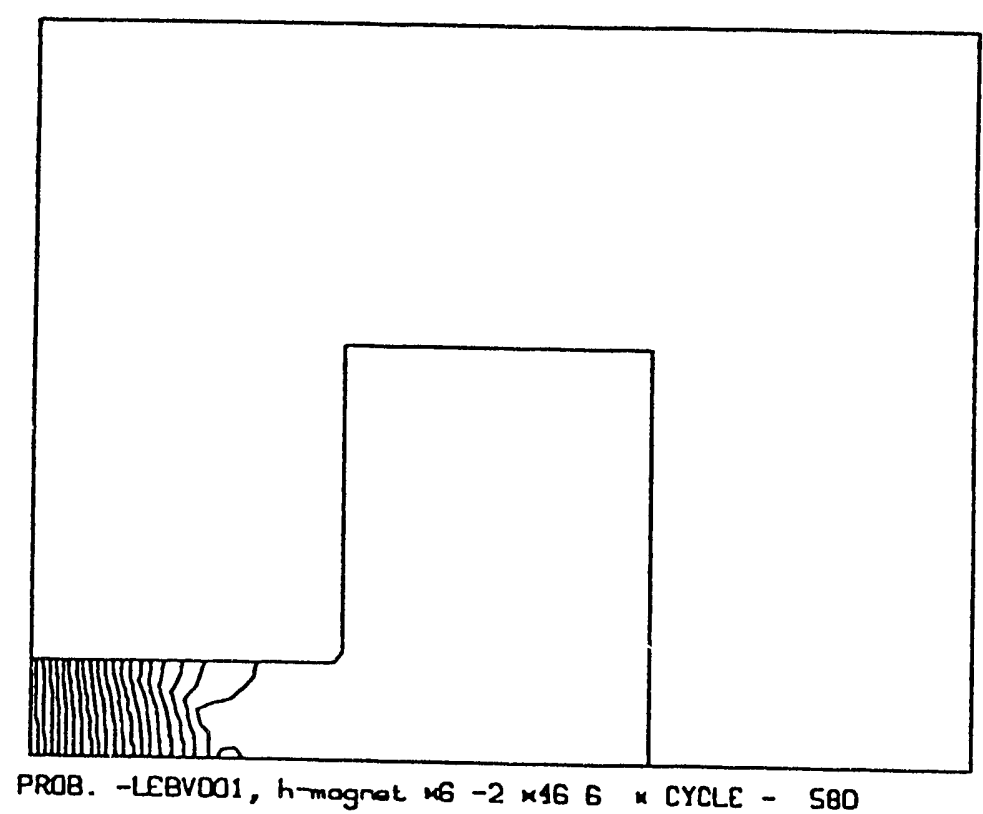


LEAST SQUARES EDIT OF PROBLEM , CYCLE 580

' $H$ ' MAG SYMMETRY TYPE

\begin{tabular}{|c|c|c|c|c|c|c|c|c|c|}
\hline CURRENT & EILAMENT & AT & 12 & 1,1 & 5.50 & 0.001. & HAS & 3.4300 & AMPS \\
\hline CURRENT & FILAMENT & AT & 11 & 4,1 & 5.00 & 1.141 , & HAS & 4.7800 & AMPS \\
\hline CURRENT & EILAMENT & AT & 8 & 5,1 & 3.75 & 2.001 & HAS & 1.0350 & AMPS \\
\hline CURRENT & EILAMENT & $\mathrm{AT}$ & 9 & 5,1 & 4.00 & 1.891. & HAS & 1.7250 & AMPS \\
\hline CURRENT & FILAMENT & $\mathrm{AT}$ & 10 & 5,1 & 4.50 & $1.58)$, & HAS & 2.8120 & AMPS \\
\hline CURRENT & FIIAMENT & $A T$ & 7 & 6.1 & 3.00 & 2.311 & HAS & 1.5800 & AMPS \\
\hline CURRENT & FILAMENT & AT & 8 & 6,1 & 3.50 & 2.121 & HAS & 1.4160 & AMPS \\
\hline CURRENT & FIIAAMENT & $A T$ & 1 & 7,1 & 0.00 & 2.751 & HAS & 0.0000 & AMPS \\
\hline CORRENT & EIIAMENT & AT & 2 & 7. 1 & 0.50 & 2.741 & HAS & 0.2500 & AMPS \\
\hline CURRENT & EILAMENT & AT & 3 & 7.1 & 1.00 & $2.70)$ & HAS & 0.5030 & AMPS \\
\hline CURRENT & FILAMENT & AT & 4 & 7. 1 & 1.50 & 2.651 & MAS & 0.7580 & AMPS \\
\hline CURRENT & EILAMENT & $A T$ & 5 & 7,1 & 2.00 & $2.56)$ & HAS & 1.0200 & AMPS \\
\hline CURRENT & FILAMENT & AT & 6 & 7,1 & 2.50 & $2.45)$ & HAS & 1.2910 & AMPS \\
\hline
\end{tabular}

STORED ENERGY $=3.2568 E-04$ JOULES / METER OR RADIAN

$X J F A C T=1.000000$

\begin{tabular}{|c|c|c|c|c|c|c|c|}
\hline K & $L$ & A (VECTOR) & $x$ & $\mathbf{Y}$ & BX (GAUSS) & BY (GAUSS) & BT (GAUSS) \\
\hline 1 & 1 & $0.000000 \mathrm{E}+00$ & 0.00000 & 0.00000 & 0.000 & -8.539 & 8.539 \\
\hline 2 & 1 & $4.293969 E+00$ & 0.50377 & 0.00000 & 0.000 & -8.492 & 8.492 \\
\hline 3 & 1 & $8.540224 \mathrm{E}+00$ & 1.00755 & 0.00000 & 0.000 & -8.352 & 8.352 \\
\hline 4 & 1 & $1.269386 \mathrm{E}+01$ & 1.51132 & 0.00000 & 0.000 & -8.125 & 8.125 \\
\hline 5 & 1 & $1.671217 \mathrm{E}+01$ & 2.01509 & 0.00000 & 0.000 & -7.816 & 7.816 \\
\hline 6 & 1 & $2.055655 E+01$ & 2.51887 & 0.00000 & 0.000 & -7.435 & 7.435 \\
\hline 7 & 1 & $2.419223 E+01$ & 3.02264 & 0.00000 & 0.000 & -6.987 & 6.987 \\
\hline 8 & 1 & $2.758604 E+01$ & 3.52642 & 0.00000 & 0.000 & -6.474 & 6.474 \\
\hline 9 & 1 & $3.070296 E+01$ & 4.03019 & 0.00000 & 0.000 & -5.886 & 5.886 \\
\hline 10 & 1 & $3.351033 E+01$ & 4.53396 & 0.00000 & 0.000 & -5.279 & 5.279 \\
\hline 11 & 1 & $3.613195 E+01$ & 5.03774 & 0.00000 & 0.000 & -4.798 & 4.798 \\
\hline 12 & 1 & $3.951679 E+01$ & 5.50000 & 0.00000 & 0.000 & -1.074 & 1.074 \\
\hline 13 & 1 & $3.744845 E+01$ & 6.04528 & 0.00000 & 0.000 & 1.655 & 1.655 \\
\hline 1 & 2 & $0.000000 E+00$ & 0.00000 & 0.43878 & 0.000 & -8.575 & 3.575 \\
\hline 2 & 2 & $2.594663 E+00$ & 0.30278 & 0.44057 & 0.050 & -3.558 & 8.558 \\
\hline
\end{tabular}


TABLE FOR FIELD COEFFICIENTS

NORMALIZATION RADIUS $=4.50000$
$\begin{array}{cccc}(B X-I B Y)=I * S U M & N *(A N+I B N) / R *(2 / R) * *(N-1) \\ N & N(A N) / R & N(B N) / R & A B S(N(C N) / R) \\ 1 & 8.5375 E+00 & 0.0000 E+00 & 8.5375 E+00 \\ 3 & -1.1488 E+00 & 0.0000 E+00 & 1.1488 E+00 \\ 5 & 5.6757 E-02 & 0.0000 E+00 & 5.6757 E-02 \\ 7 & 7.6619 E-03 & 0.0000 E+00 & 7.6619 E-03 \\ 9 & -2.2467 E-03 & 0.0000 E+00 & 2.2467 E-03\end{array}$




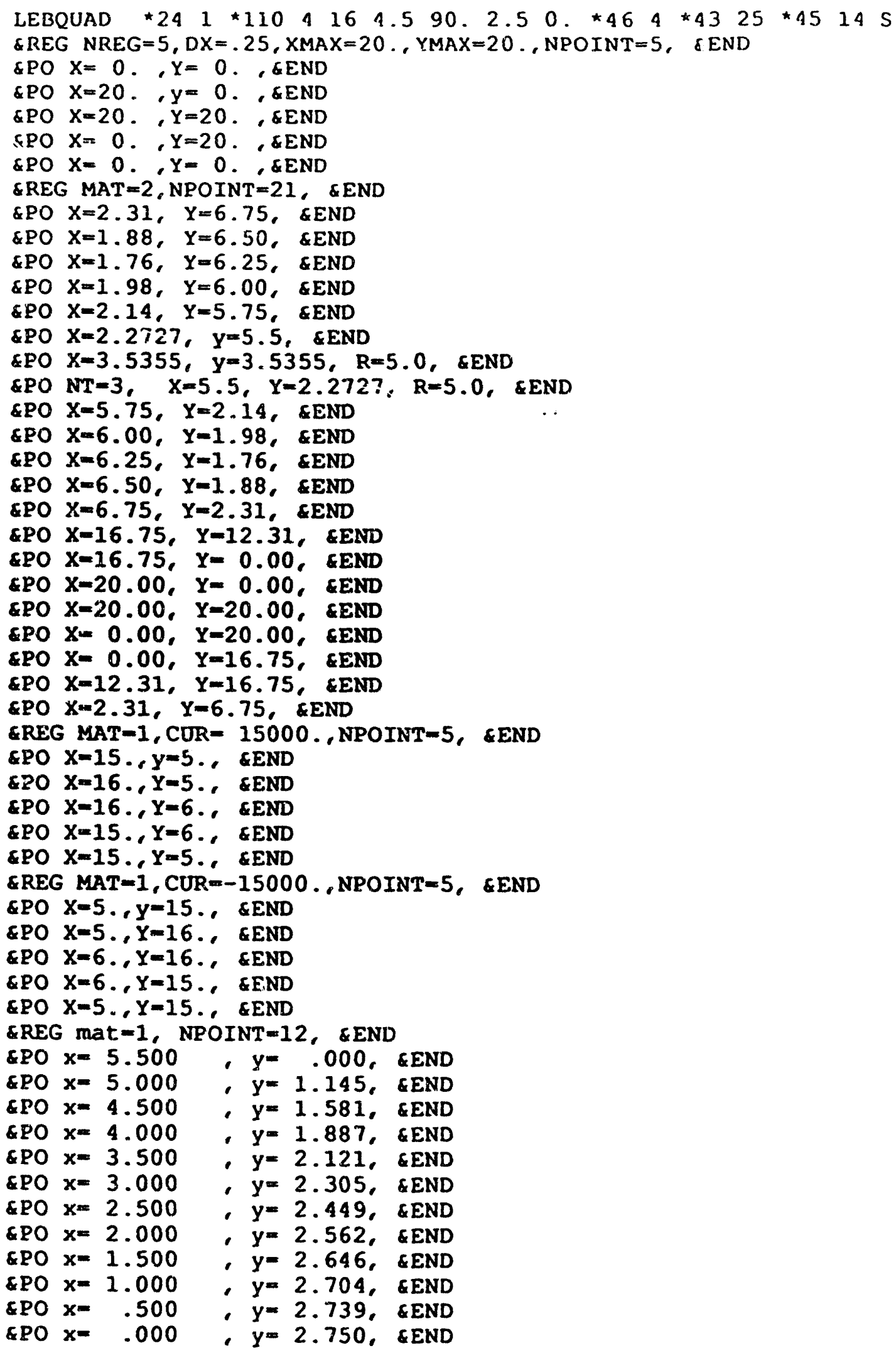




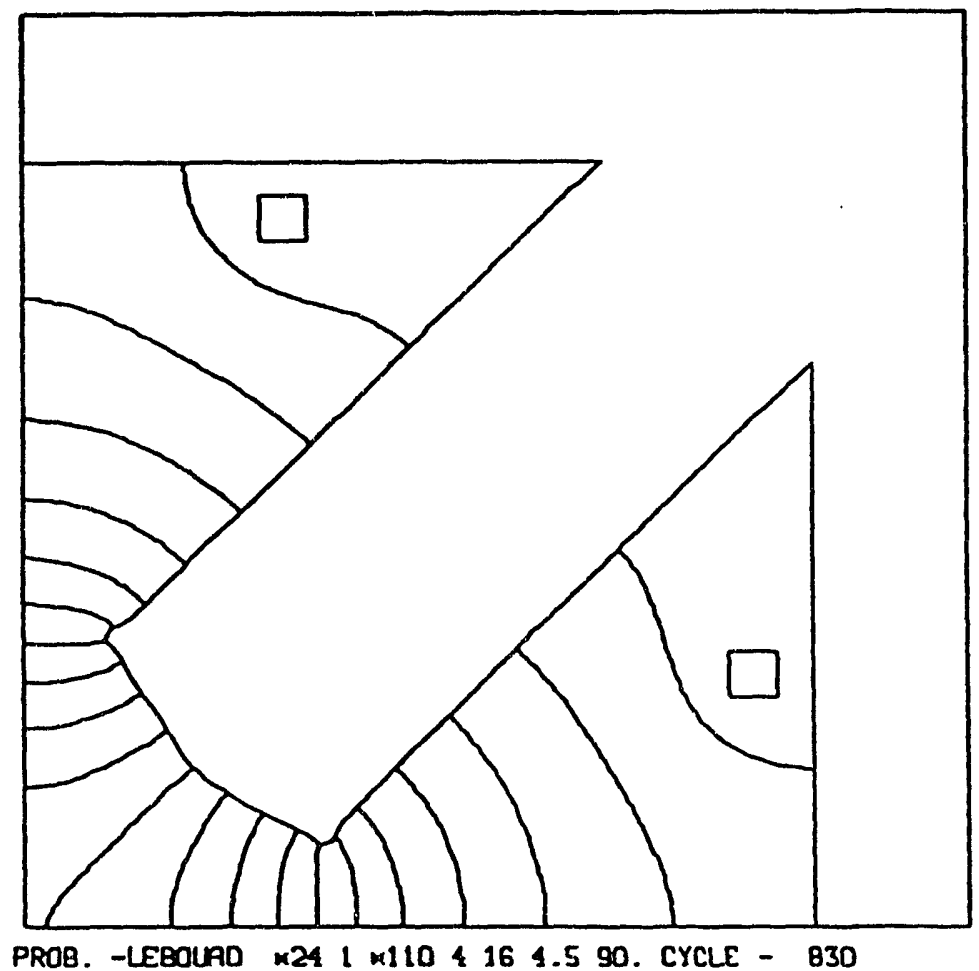

PROB. -LEBOLAD $\times 241$ M110\&16 4.590 . CYCLE - 830 
LEAST SQUARES EDIT OF PROBLEM, CYCLE 830

SYMM QUA SYMMETRY TYPE

STORED ENERGY $=1.1441 E+03$ JOULES / METER OR RADIAN

$\left|\frac{B^{*}(z) \cdot r_{p}}{i \partial z}\right|=3730 G=1 ;$ $X \mathrm{JFACT}=1.000000$

$$
\mathrm{K}
$$$$
3
$$$$
311.770382 \mathrm{E}+03
$$$$
412.002833 E+03
$$$$
512.328308 E+03
$$$$
612.746965 \mathrm{E}+03
$$$$
713.258947 \mathrm{E}+03
$$$$
813.864412 E+03
$$$$
914.563541 E+03
$$$$
1015.356534 \mathrm{E}+03
$$$$
1116.243606 E+03
$$$$
1217.224970 \mathrm{E}+03
$$$$
1318.300819 E+03
$$$$
1419.471295 E+03
$$$$
1511.073646 E+04
$$$$
1611.209628 \mathrm{E}+04
$$$$
1711.355052 \mathrm{E}+04
$$$$
1811.509865 E+04
$$$$
1911.673963 E+04
$$$$
2011.847151 E+04
$$$$
2112.029095 E+04
$$$$
2212.219244 E+04
$$$$
2312.416712 E+04
$$$$
2412.620199 E+04
$$$$
2512.827732 E+04
$$$$
121.549517 E+03
$$$$
121.549517 E+03
$$$$
221.567763 E+03
$$

$321.660277 E+03$

$x \quad Y$

$0.00000 \quad 0.00000$

0.25000

0.50000

0.75000

1.00000

1.25000

1.50000

1.75000

2.00000

2.25000

2.50000

2.75000

3.00000

3.25000

3.50000

3.75000

4.00000

4. 25000

4.50000

4.75000

5.00000

5.25000

5.50000

5.75000

6.00000

0.00000

0.15055

0.38595
0.00000

0.00000

0.00000

0.00000

0.00000

0.00000

0.00000

0.00000

0.00000

0.00000

0.00000

0.00000

0.00000

0.00000

0.00000

0.00000

0.00000

0.00000

0.00000

0.00000

0.00000

0.00000

0.00000

0.21739

0.21789

0.21863
0.00000

\section{BX (GAUSS)}

$$
0.000
$$

0.000

0.000

0.000

0.000

0.000

0.000

0.000

0.000

0.000

0.000

0.000

0.000

0.000

0.000

0.000

0.000

0.000

0.000

0.000

0.000

0.000

0.000

0.00

0.000

$-327.655$

$-325.990$

$-321.901$
BY (GAUSS)

0.000

$-373.036$

$-744.133$

$-1115.688$

$-1488.121$

$-1861.135$

$-2234.747$

$-2609.036$

$-2984.091$

$-3359.980$

$-3736.732$

$-4114.310$

$-4492.572$

$-4871: 272$

$-5250.039$

$-5628.312$

$-6005.197$

$-6379.128$

$-6747.384$

$-7105.525$

$-7446.651$

$-7760.056$

$-8030.212$

$-8234.813$

$-8351.120$

0.000

$-225.160$

$-5 i \div .262$
BT (GAUSS) DB' -

373.036

744.133

1115.688

1488.121

1861.135

2234.747

2609.036

2984.091

3359.980

3736.732

4114.310

4492.572

4871.272

5250.039

5628.312

6005.197

6379.128

6747.384

7105.525

7446.651

7760.056

8030.212

8234.813

8351.120

327.655

306.190 $50.8: 3$ 


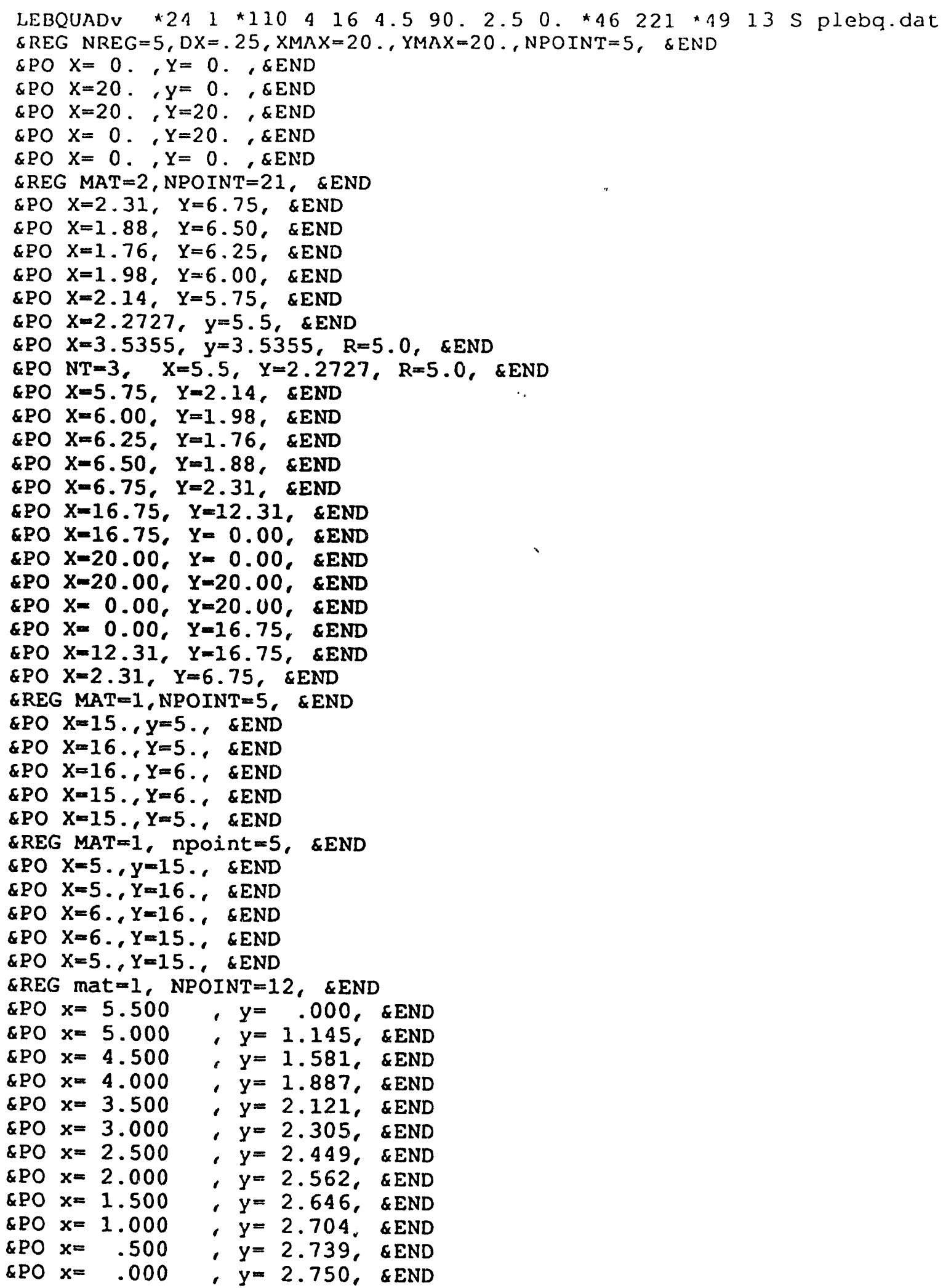




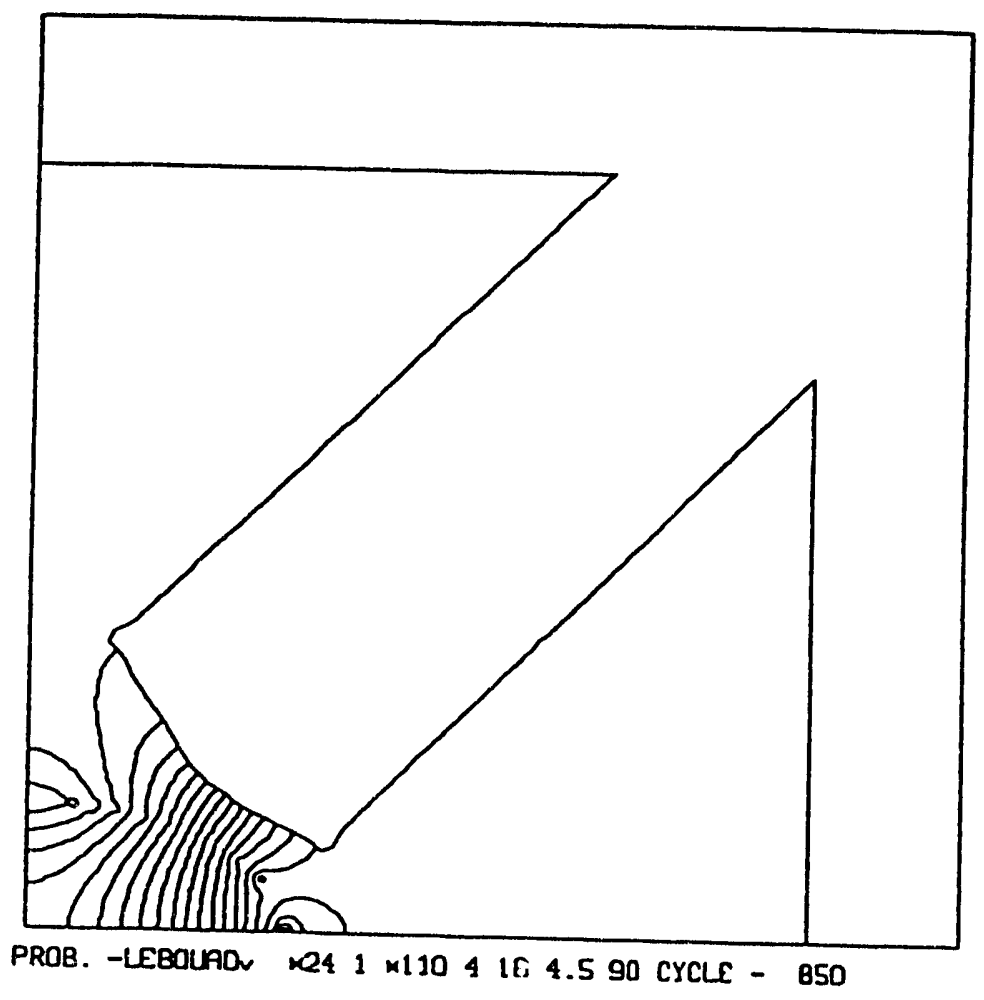




\begin{tabular}{|c|c|c|c|c|c|c|c|c|c|c|}
\hline LEAST SC & QUARES ED & IT OF & PRO & BLEM & ' & YCLE & 860 & & & \\
\hline EDIT IT & YPE 221 & & & & & & & & & \\
\hline CURRENT & FILAMENT & AT & 23 & 1, & 1 & 5.50 & 0.001 . & HAS & 2.7709 & AMPS \\
\hline CURRENT & F ILAMENT & $\mathrm{AT}$ & 21 & 6, & 1 & 5.00 & 1.141. & HAS & 3.0119 & AMPS \\
\hline CURRENT & FILAMENT & AT & 19 & 8 & 1 & 4.50 & 1.581 , & HAS & 1.2112 & AMPS \\
\hline CURRENT & FILAMENT & $A T$ & 16 & 10 & 1 & 3.75 & 2.001 & HAS & 0.1045 & AMPS \\
\hline CURRENT & FILAMENT & AT & 17 & 10 & 1 & 4.00 & 1.891. & HAS & 0.3722 & AMPS \\
\hline CURRENT & FILAMENT & $\mathrm{AT}$ & 15 & 11 & 1 & 3.50 & 2.121 & HAS & -0.0344 & AMPS \\
\hline CURRENT & FILAMENT & AT & 11 & 12 . & 1 & 2.50 & $2.45)$ & HAS & -0.8184 & AMPS \\
\hline CURRENT & EILAMENT & AT & 13 & 12. & 1 & 3.00 & 2.311 & HAS & -0.4739 & AMPS \\
\hline CURRENT & FILAMENT & $A T$ & 5 & 13, & 1 & 1.00 & 2.701 & HAS & -1.4442 & AMPS \\
\hline CORRENT & FILAMENT & AT & 7 & 13. & 1 & 1.50 & 2.651 . & HAS & -1.2979 & AMPS \\
\hline CURRENT & FILAMENT & AT & 9 & 13. & 1 & 2.00 & $2.56)$ & HAS & -1.0904 & AMPS \\
\hline CURRENT & FILAMENT & AT & 1 & 14, & 1 & 0.00 & 2.751 , & HAS & -0.7805 & AMPS \\
\hline CURRENT & FILAMENT & AT & 3 & 14. & 1 & 0.50 & 2.741 & HAS & -1.5311 & AMPS \\
\hline
\end{tabular}
STORED ENERGY $=4.5703 E-05$ JOULES / METER OR RADIAN $\mathrm{XJFACT}=1.000000$

\begin{tabular}{|c|c|c|c|c|c|c|}
\hline K & $A(V E C T O R)$ & $\mathrm{x}$ & $Y$ & BX (GAUSS) & BY (GAUSS) & BT (GAUSS) \\
\hline 1 & $1-3.775190 E+00$ & 0.00000 & 0.00000 & 0.000 & 0.000 & 0.000 \\
\hline 2 & $1-3.748710 E+00$ & 0.25000 & 0.00000 & 0.000 & -0.204 & 0.204 \\
\hline 3 & $1-3.672503 E+00$ & 0.50000 & 0.00000 & 0.000 & -0.405 & 0.405 \\
\hline 4 & $1-3.545983 E+00$ & 0.75000 & 0.00000 & 0.000 & -0.607 & 0.607 \\
\hline 5 & $-3.369107 \mathrm{E}+00$ & 1.00000 & 0.00000 & 0.000 & -0.808 & 0.808 \\
\hline 6 & $-3.142082 \mathrm{E}+00$ & 1.25000 & 0.00000 & 0.000 & -1.008 & 1.008 \\
\hline 7 & $-2.865236 E+00$ & 1.50000 & 0.00000 & 0.000 & -1.206 & 1.206 \\
\hline 8 & $-2.539022 E+00$ & 1.75000 & 0.00000 & 0.000 & -1.403 & 1.403 \\
\hline 9 & $-2.164057 E+00$ & 2.00000 & 0.00000 & 0.000 & -1.596 & 1.596 \\
\hline 10 & $1-1.741158 \mathrm{E}+00$ & 2.25000 & 0.00000 & 0.000 & -1.786 & 1.786 \\
\hline 11 & $-1.271398 E+00$ & 2.50000 & 0.00000 & 0.000 & -1.971 & 1.971 \\
\hline 12 & $-7.561750 E-01$ & 2.75000 & 0.00000 & 0.000 & -2.150 & 2.150 \\
\hline 13 & $-1.972908 E-01$ & 3.00000 & 0.00000 & 0.000 & -2.320 & 2.320 \\
\hline 14 & $4.029561 E-01$ & 3.25000 & 0.00000 & 0.000 & -2.480 & 2. 4.8 \\
\hline 15 & $1.041664 E+00$ & 3.50000 & 0.00000 & 0.000 & -2.52 .7 & 2. $62=$ \\
\hline
\end{tabular}


'TABLE FOR FIELD COEFEICIEN'S

NORMALIZATION RADIUS $=2.50000$

$\begin{array}{cccc}(B X-I B Y)=I * \operatorname{SUM} N(A N+I B N) / R *(Z / R) *(N-1) \\ N & N(A N) / R & N(B N) / R & A B S(N(C N) / R) \\ 2 & 2.0267 E+00 & 0.0000 E+00 & 2.0267 E+00 \\ 4 & -3.9091 E-02 & 0.0000 E+00 & 3.9091 E-02 \\ 6 & -7.7138 E-03 & 0.0000 E+00 & 7.7138 E-03 \\ 8 & -1.9802 E-02 & 0.0000 E+00 & 1.9802 E-02\end{array}$




\title{
Appendix C: Code EDDY files for Eddy Current Induced Harmonic Analytical Calculations
}

\author{
1. Code EDDY \\ 2. Code EDDY_STR \\ 3. dipole vaccum chamber options \\ 4. dipole coil positioning \\ 5. vacuum chamber thickness and tolerance study \\ 6. dipole coil positioning \\ 7. quadrupole vaccum chamber options \\ 8. sextupole vaccum chamber \\ 9. miscellaneous EDDY results \\ 10. Code EDDYCONT
}




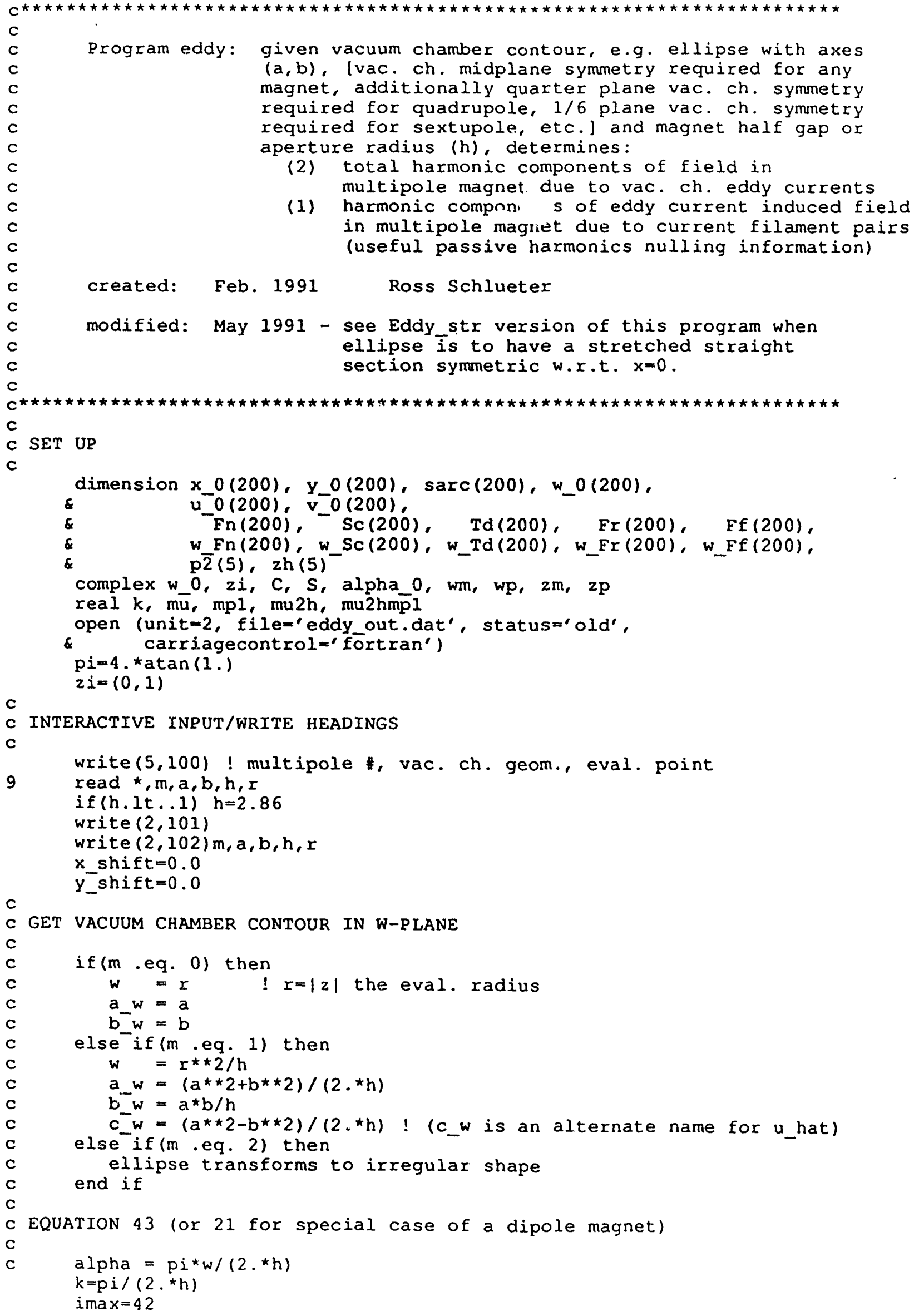




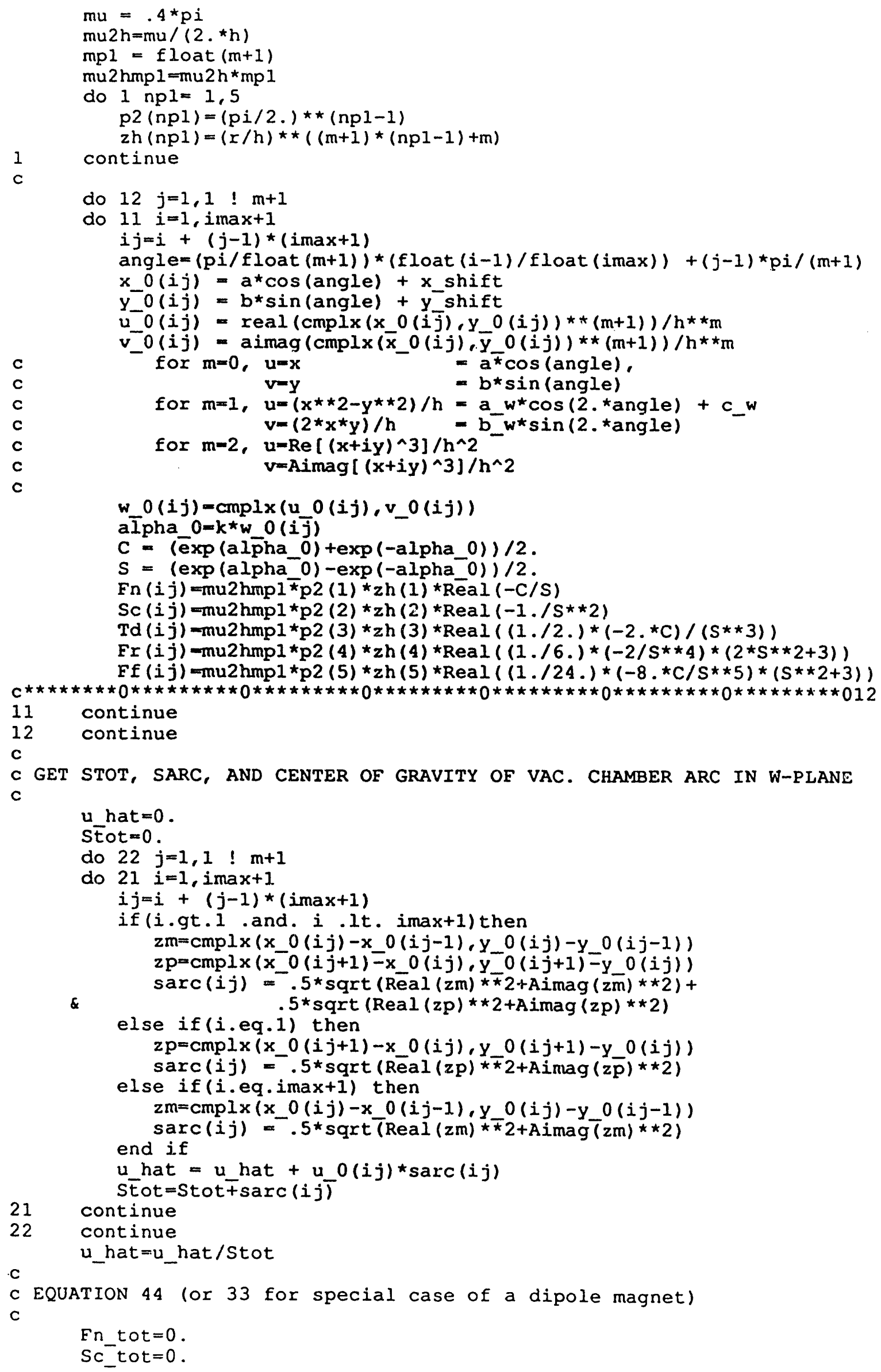




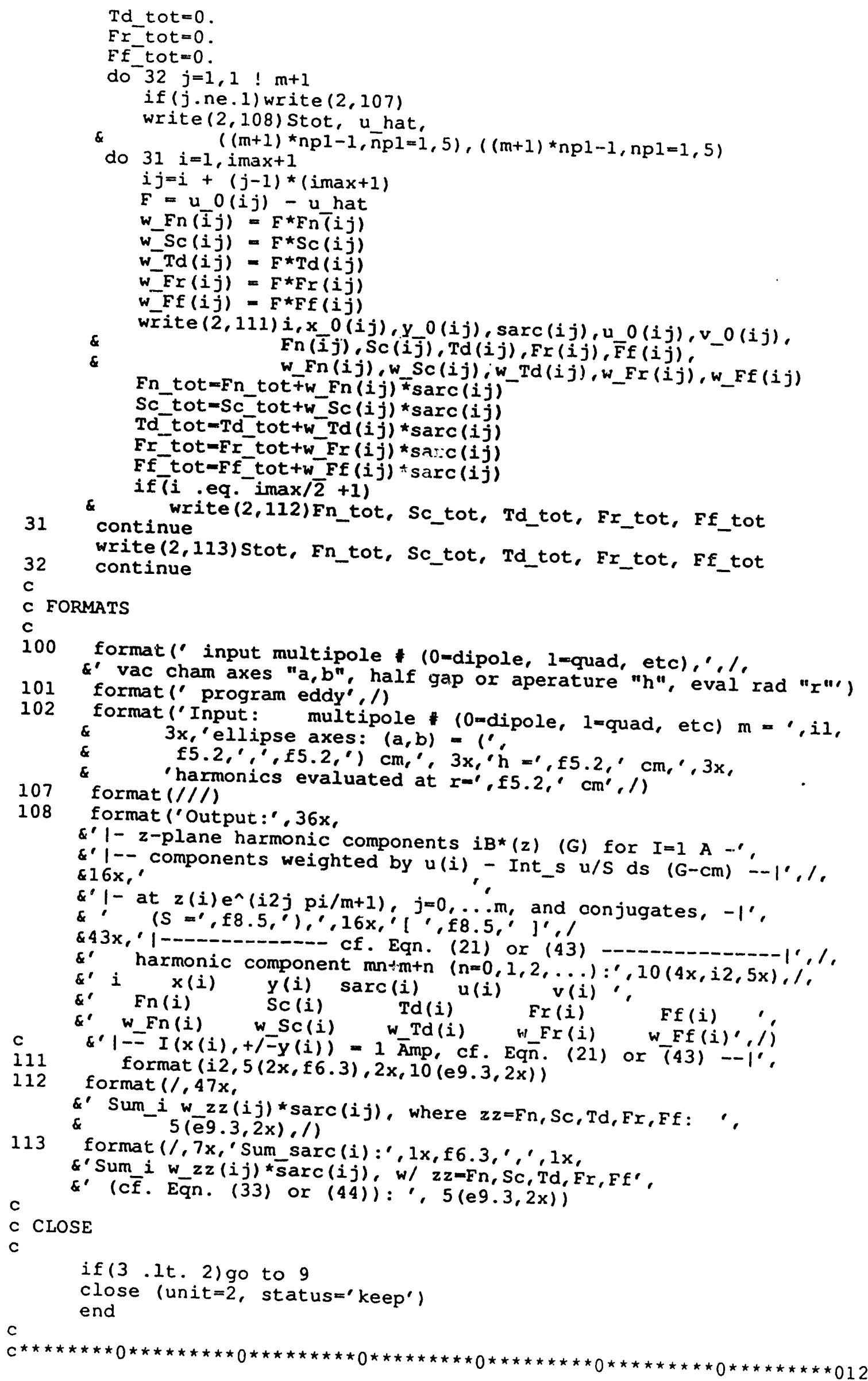


Program eddy_str: given vacuum chamber contour, e.g. ellipse with axes $(a, b)$, lvac. ch. midplane symmetry required for any magnet, additionally quarter plane vac. ch. symmetry required for quadrupole, $1 / 6$ plane vac. ch. symmetry required for sextupole, etc.J and magnet half gap or aperture radius (h), determines:

(2) total harmonic components of field in multipole magnet due to vac. ch. eddy currents

(1) harmonic components of eddy current induced field in multipole magnet due to current filament pairs (useful passive harmonics nulling information)

created: Feb. 1991 Ross Schlueter

modified: May 1991 - this Eddy_str version accomodates an added vac. ch. straight section symmetric w.r.t. $x=0$. FOR USE ONLY IN DIPOLE AND QUADRUPOLE MAGNETS; the equations below are not valid for this asymmetry condition in higher order multipoles. See eddy_elsex for special case of elliptical sextupole.

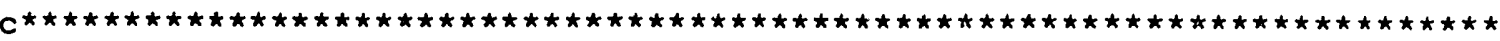

c C SET UP

c

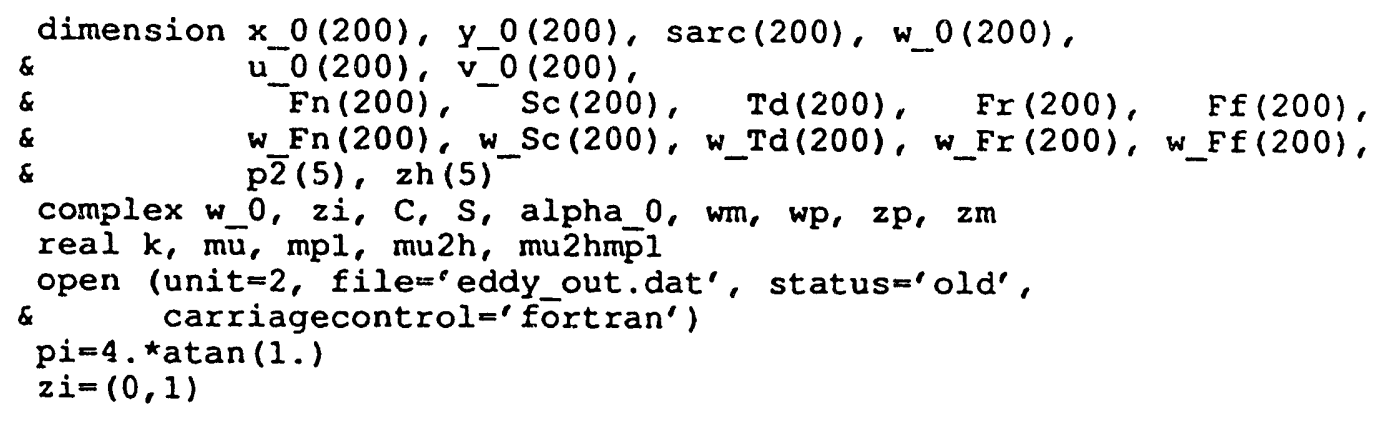

c

C INTERACTIVE INPUT/WRITE HEADINGS

c

write $(5,100)$ ! multipole \#, vac. ch. geom., eval. point

9 read *, $m, a, b, s t r, h, r$ ! str added to accomodate stretched ellipse shapes if (h.lt. . I) $h=2.86$

write $(2,101)$

write $(2,102) m, a, b, s t r, h, r$ ! str

if $(\mathrm{m} . \mathrm{gt}$. 1) then

write $(5,999)$ 999 format ('invalid symmetry condition for this code')

end if

$x$ shift $=0$.

$c$

$y_{-}$shift $=0$.

c GET VACUUM CHAMBER CONTOUR IN W-PLANE

if $(m$.eq. 0) then

$w=r \quad|r=| z \mid$ the eval. radius

$a \_w=a$

$b^{-} w=b$

else if (m.eq. 1) then

$w=r * \star 2 / h$

a_w $=(a \star \star 2+b \star \star 2) /(2 . \star h)$

$b_{-}^{-} w=a * b / h$

$c_{-}^{-} w=\left(a^{\star \star} 2-b^{\star \star} 2\right) /\left(2,{ }^{\star} h\right) !\left(c_{-} w\right.$ is an alternate name Eor u_hat) 


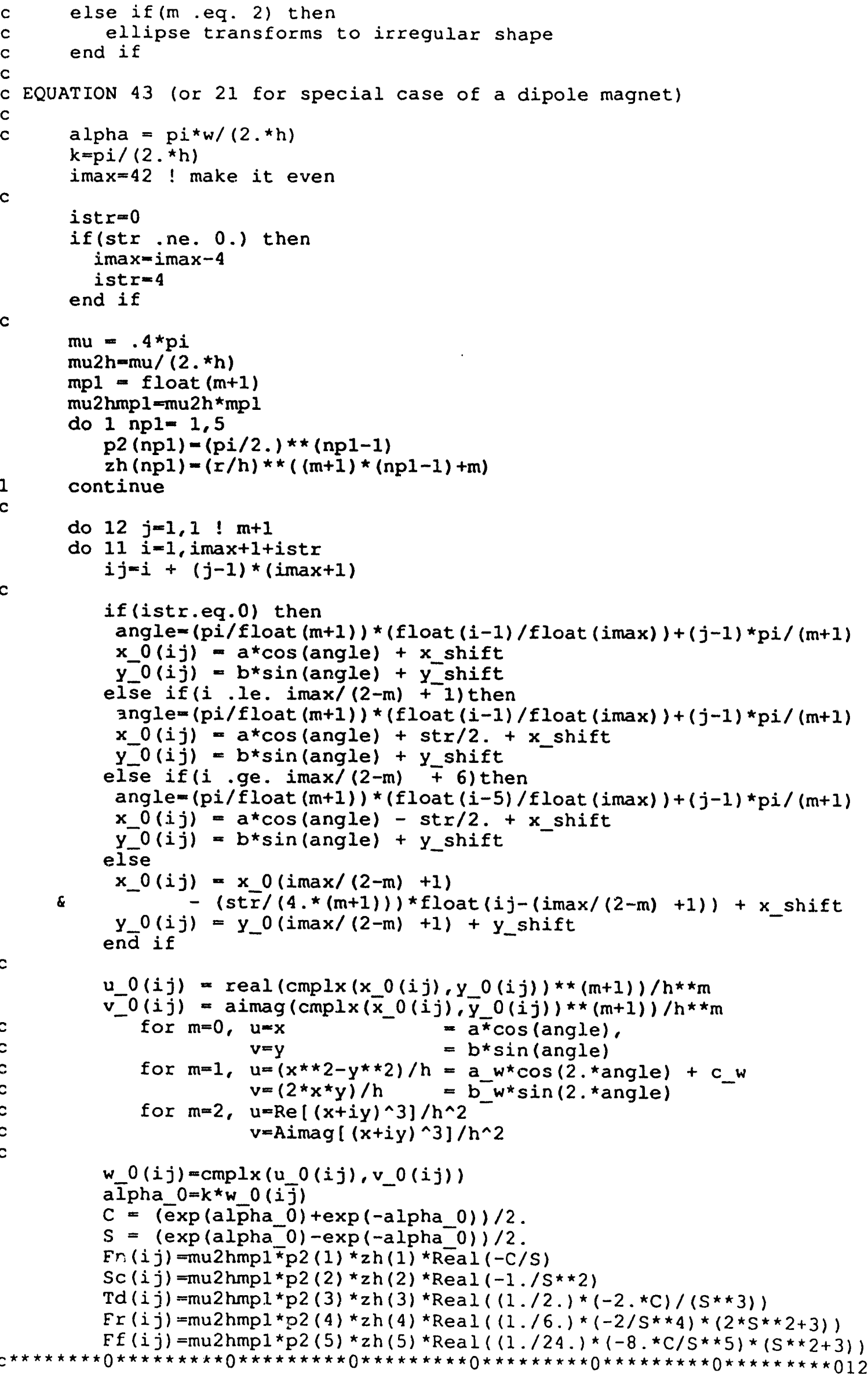




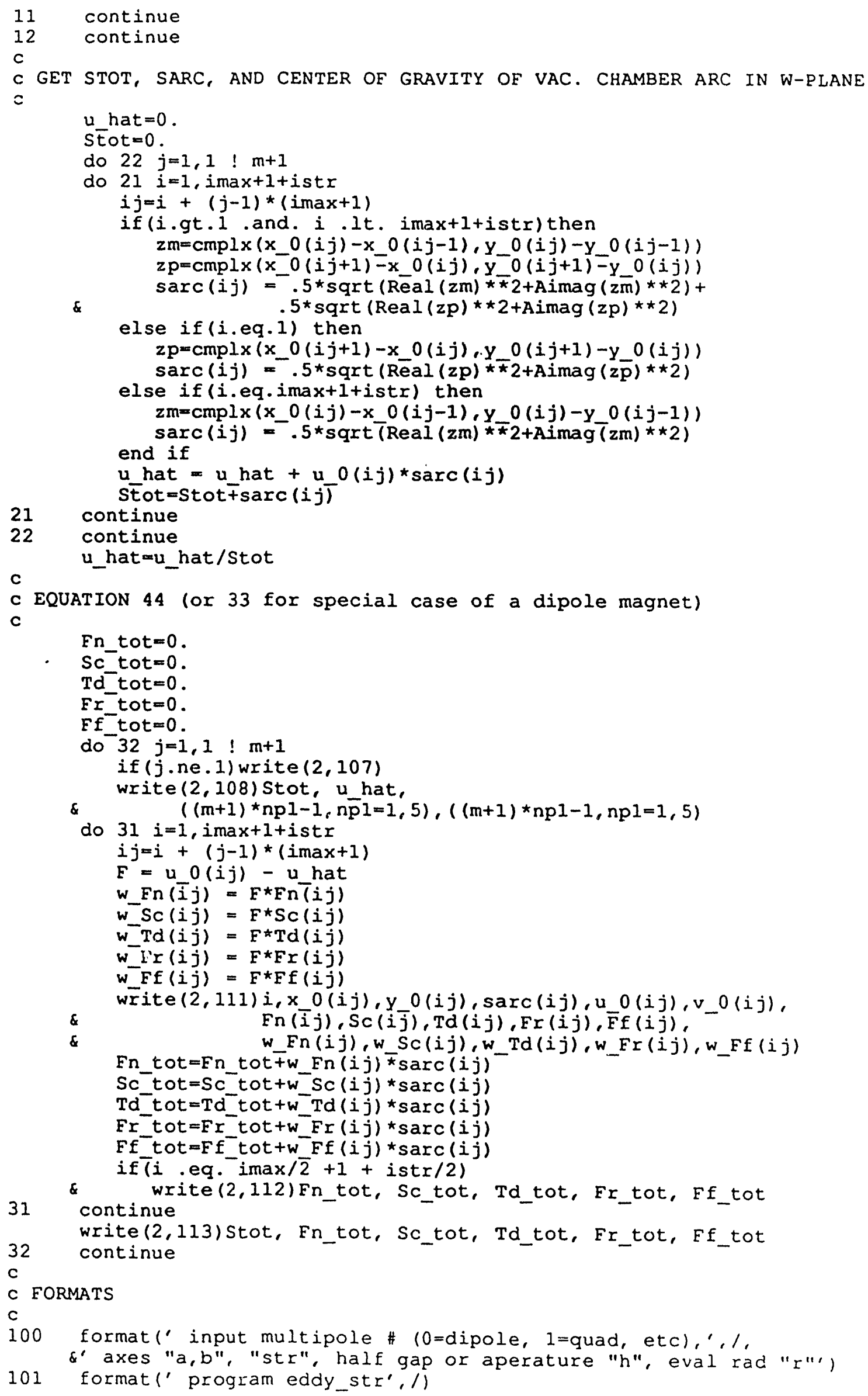




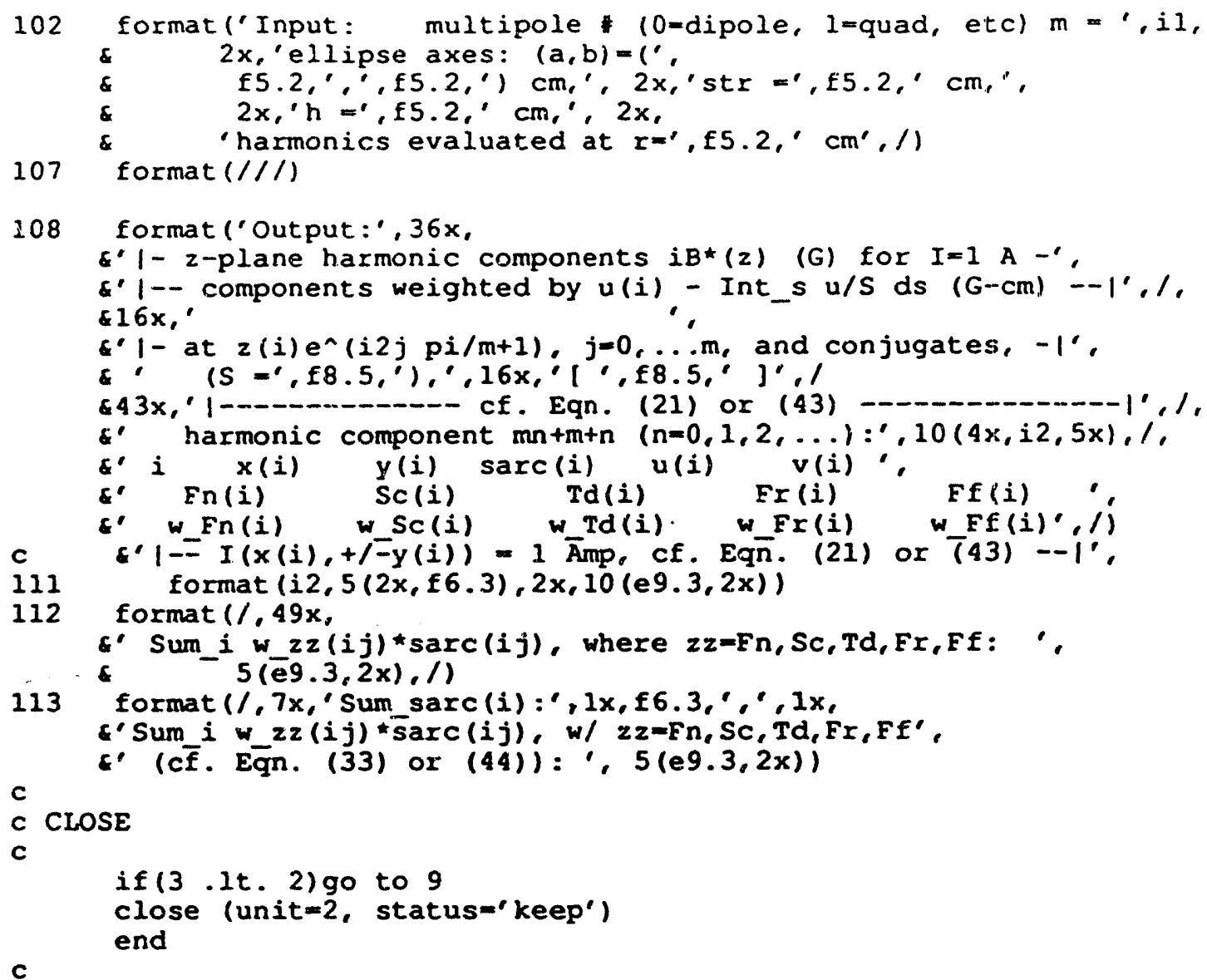




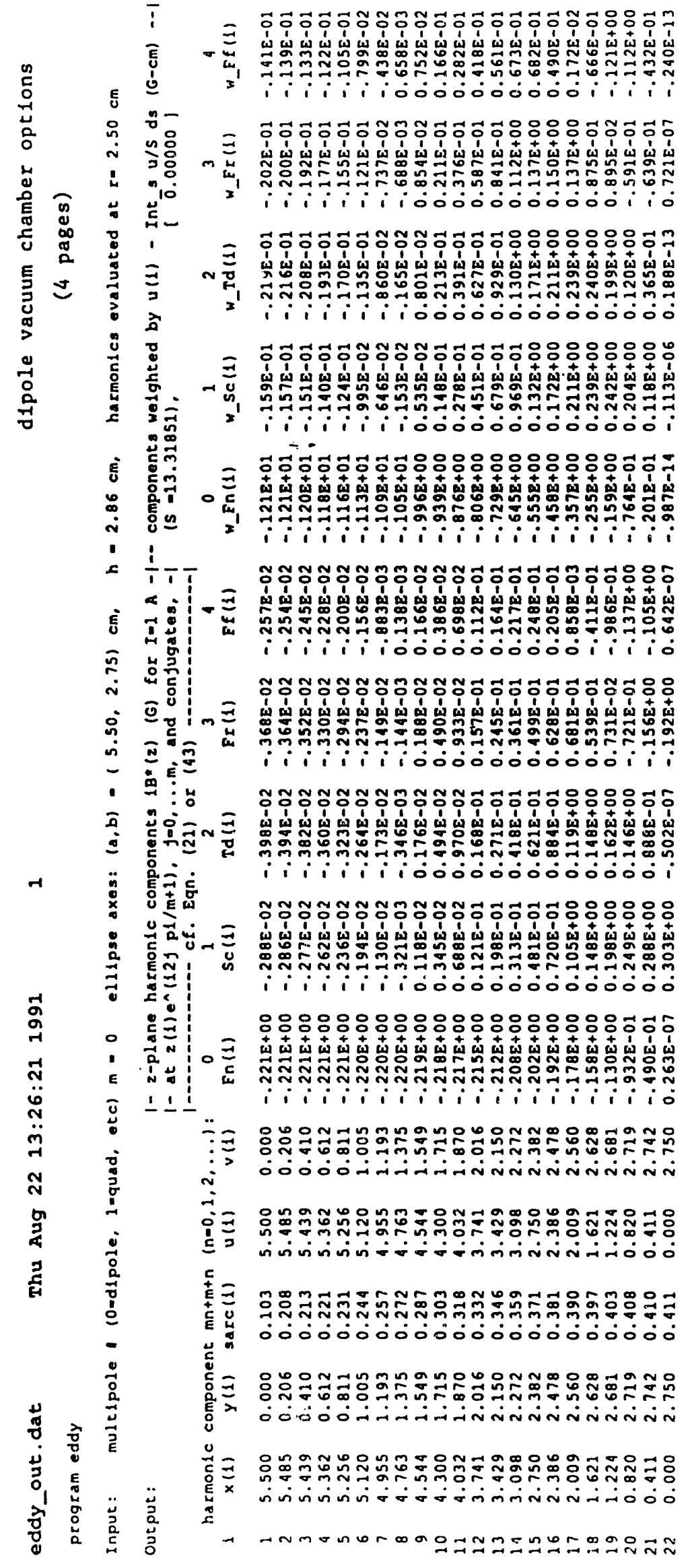

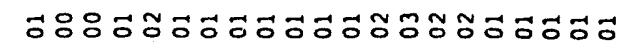

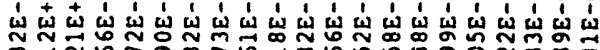

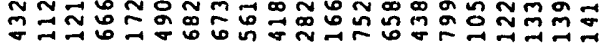

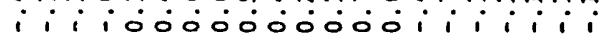

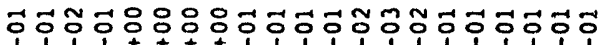

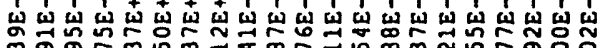

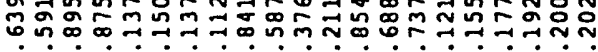
Doi i i i i i i 00000000

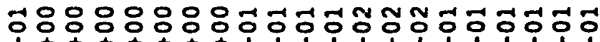

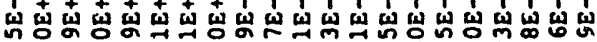

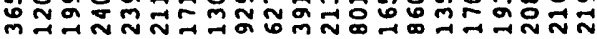
000000000000 i i i i i

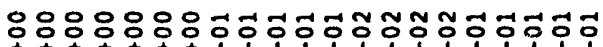

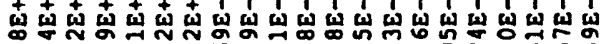

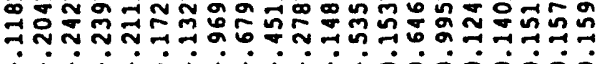

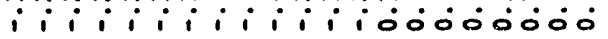

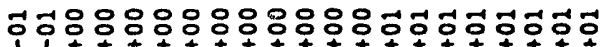

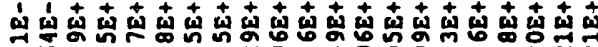

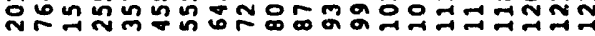
i:i i i i i i i i i i i

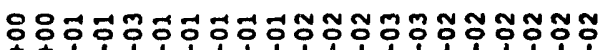

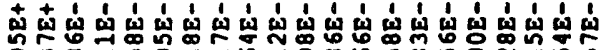

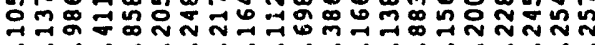
o000iili ili i io000000

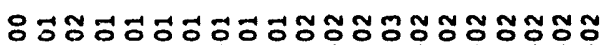

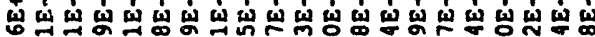

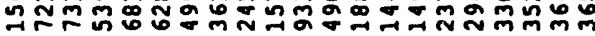
i iojosoojoóili ili

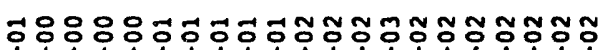

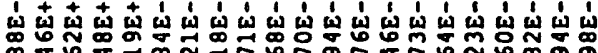

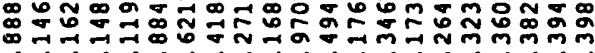
i i i i i i i i i iojo 0000

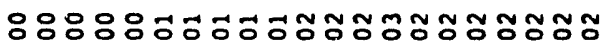

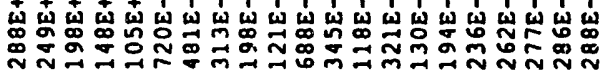
o000000000000ii iiii

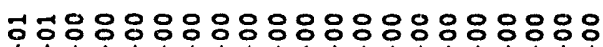

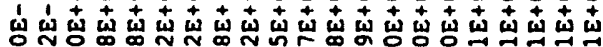

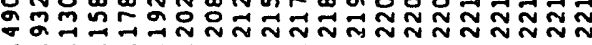
-00000000000000000000

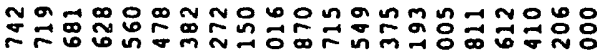

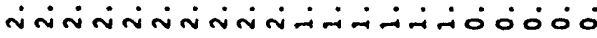

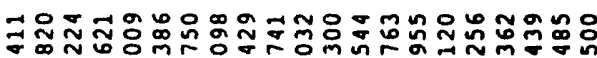

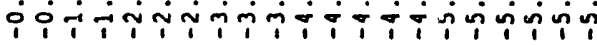

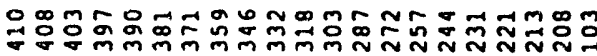
ठ0ं0ல0000000000000

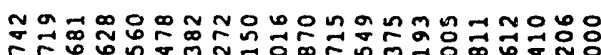

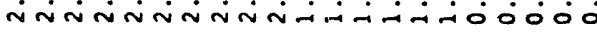

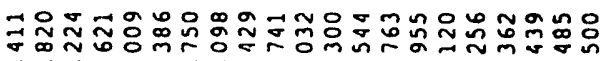

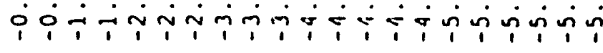

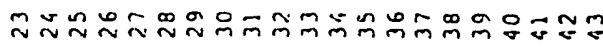




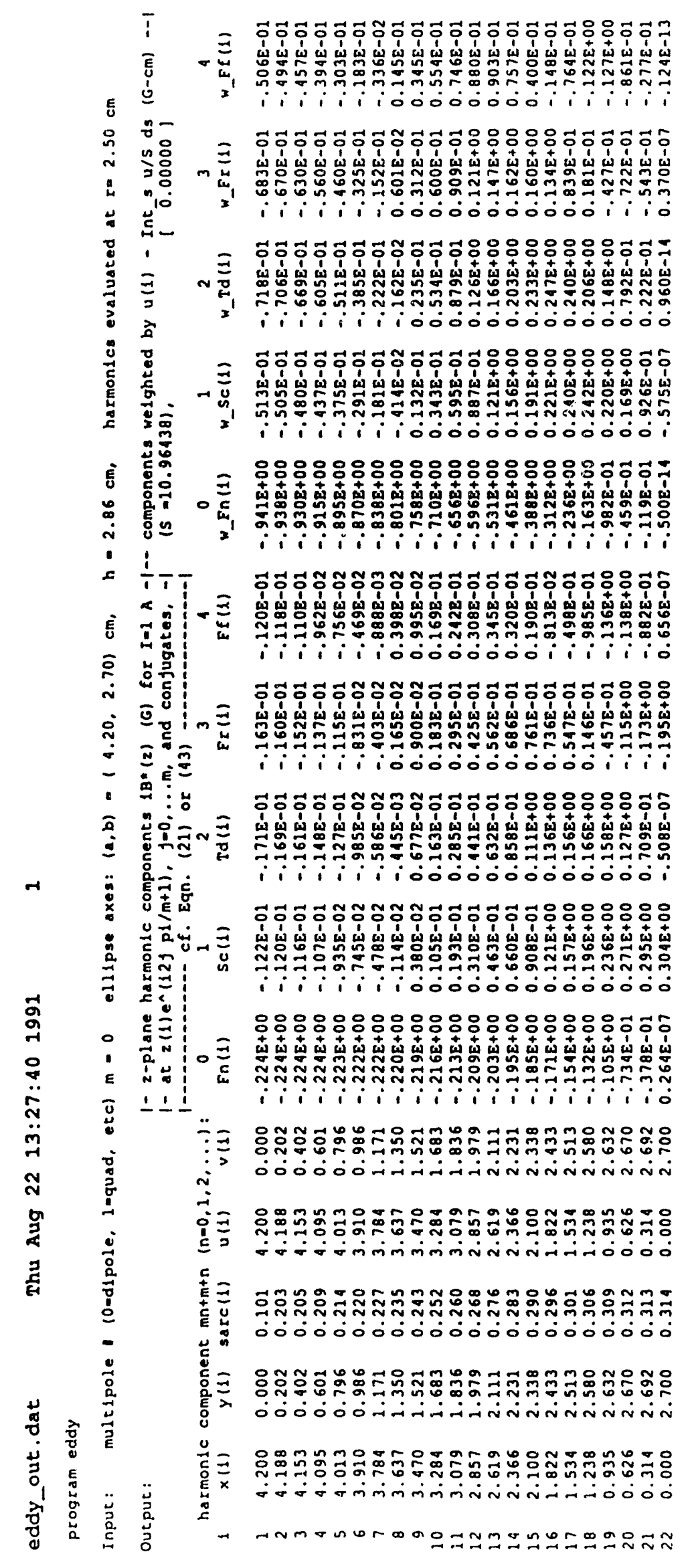

\section{ó}

\section{高}

ก

\section{$\ddot{4}$}

(5

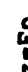

$$
\text { N }
$$

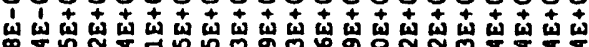

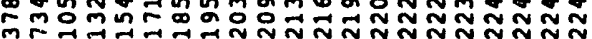
000000000000000000000

รั0

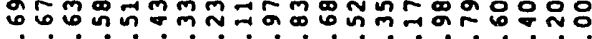

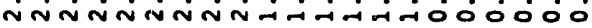

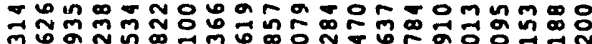

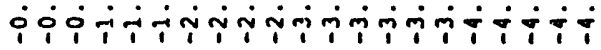

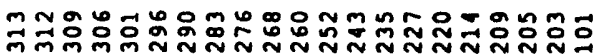
000000000000000000000

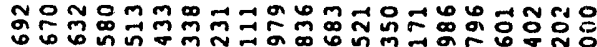

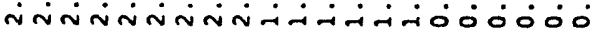

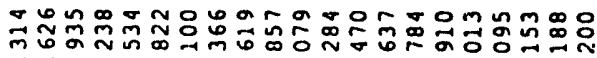

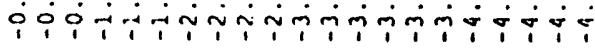

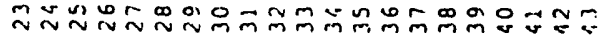




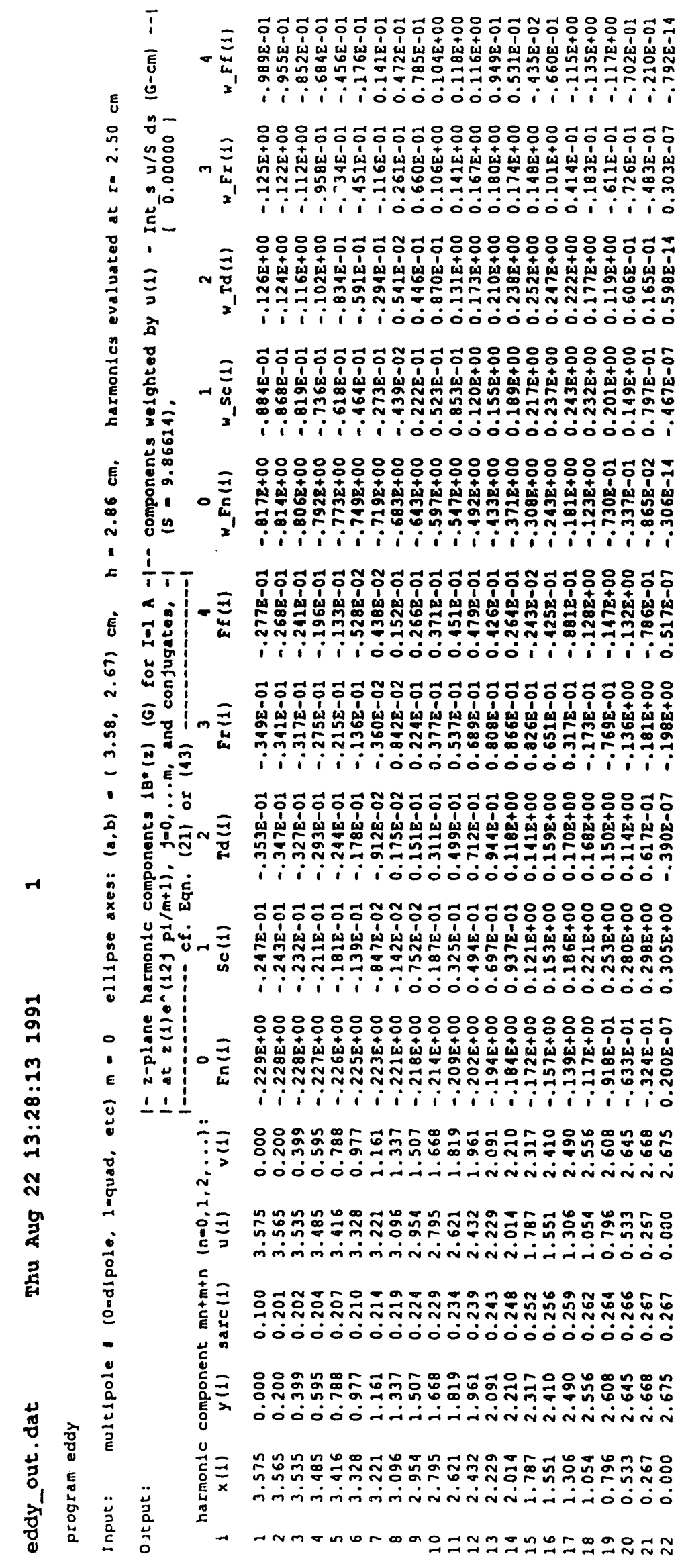

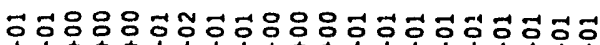

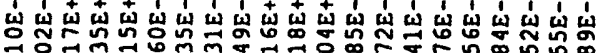

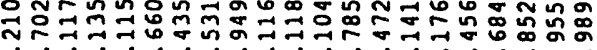

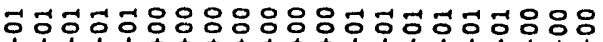

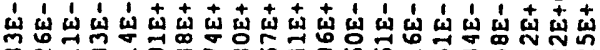

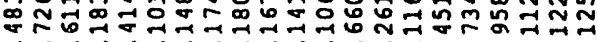

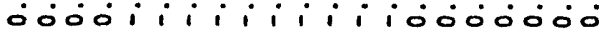

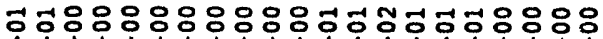

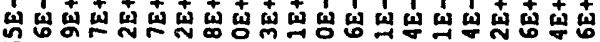

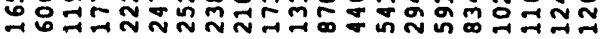
o00000000000i ilii

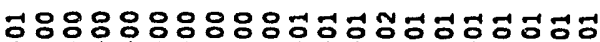

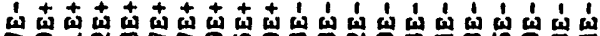

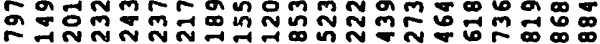

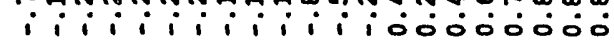

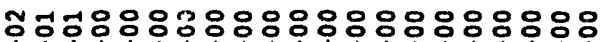

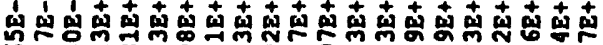

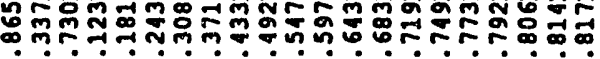
i i i i i i i i i i i i i

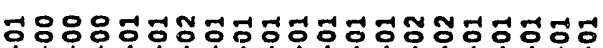

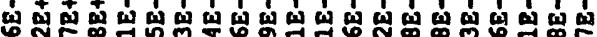

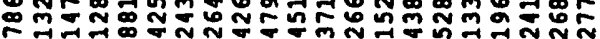

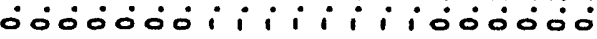

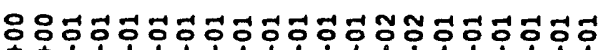

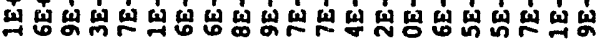

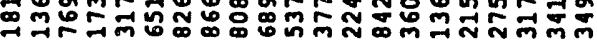
i i i0000000000i i i i i

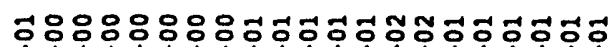

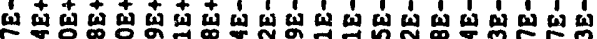

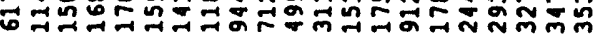
i i i i i i i jo

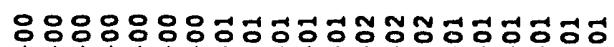

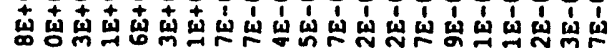

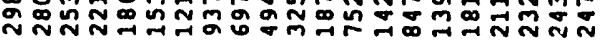
0000000000000ii i i i i

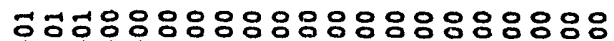

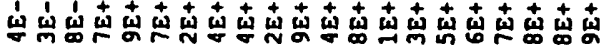
N

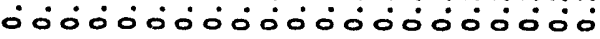

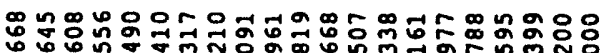

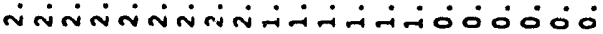

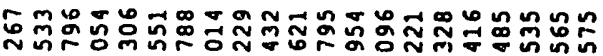

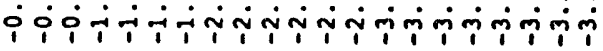

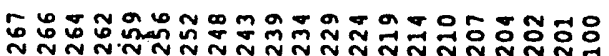
-00000000000000000

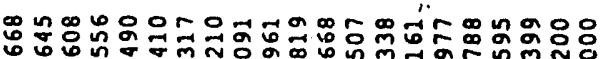

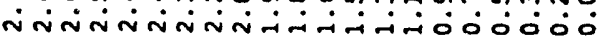

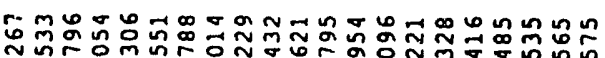

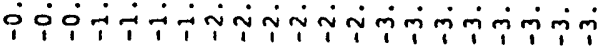

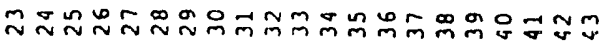




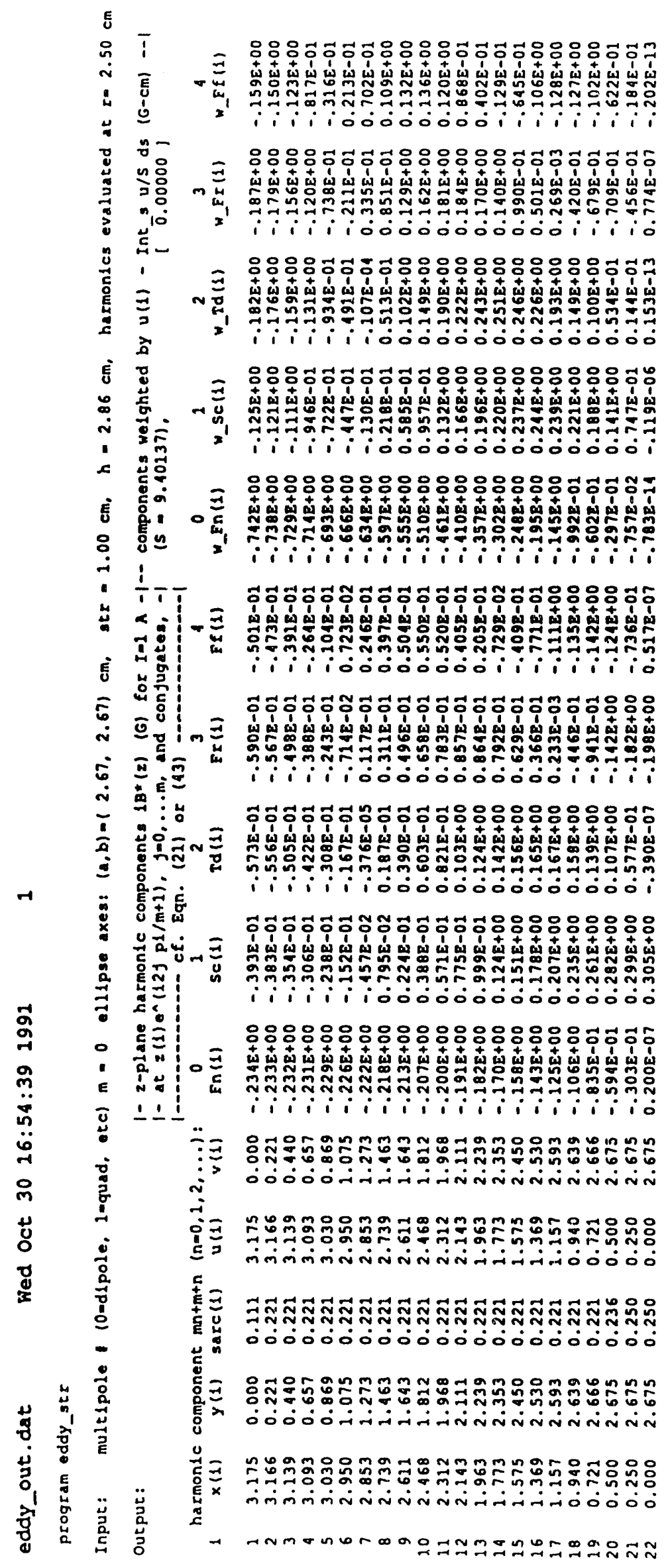

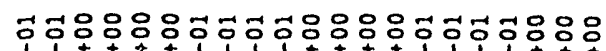

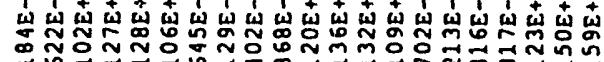

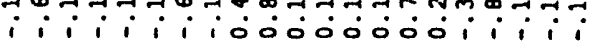

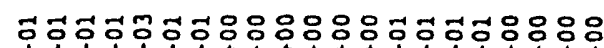

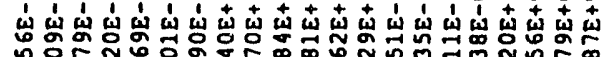

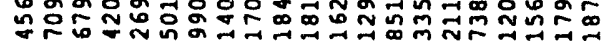
o000ii i i i i i jojo:

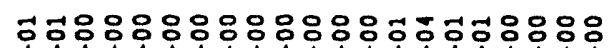

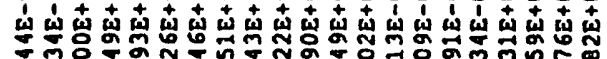

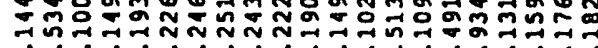
oojoojooodoóoi i i i i

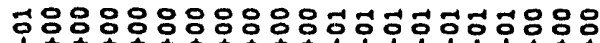

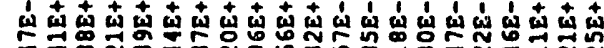

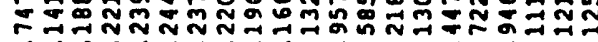
$i i i i_{i} i i_{i} i 0_{0} 00000$

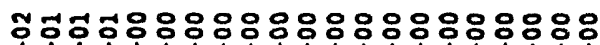

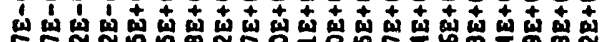
๙ $i i_{i} i i_{i} i i_{i} i_{i} i i_{i}$

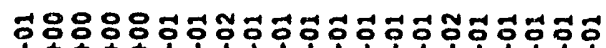

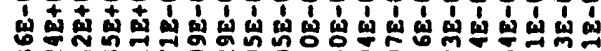

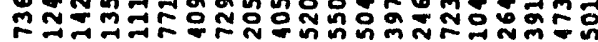

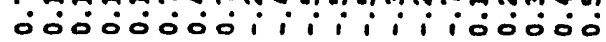

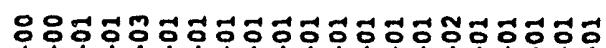

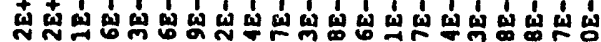

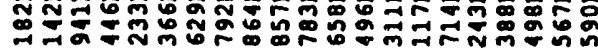
i i ióosoojosili i i

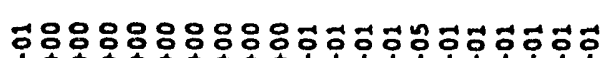

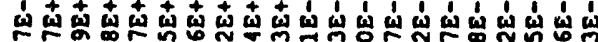

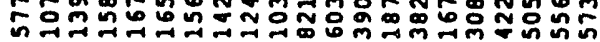
i i i i i i i i i ioojo00

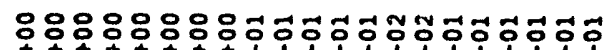

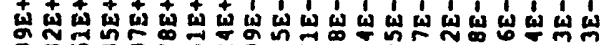

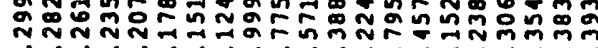
o000000000000i ili i

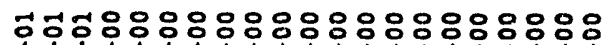

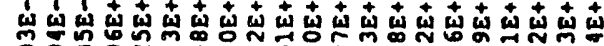

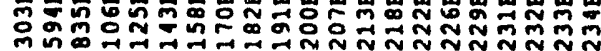

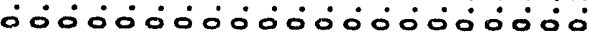

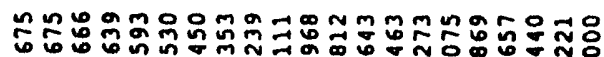

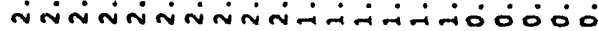

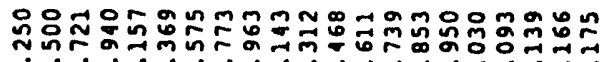

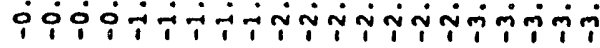

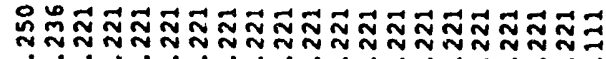
0000000000000000000

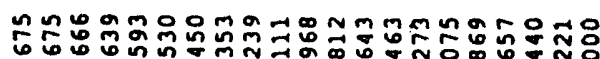

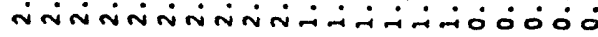

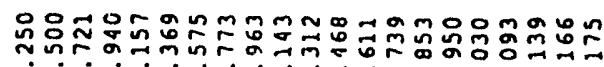

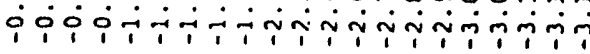

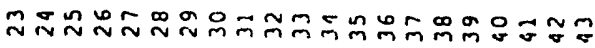

$$
\text { : }
$$

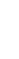
家 $\underline{\underline{u}}$ 


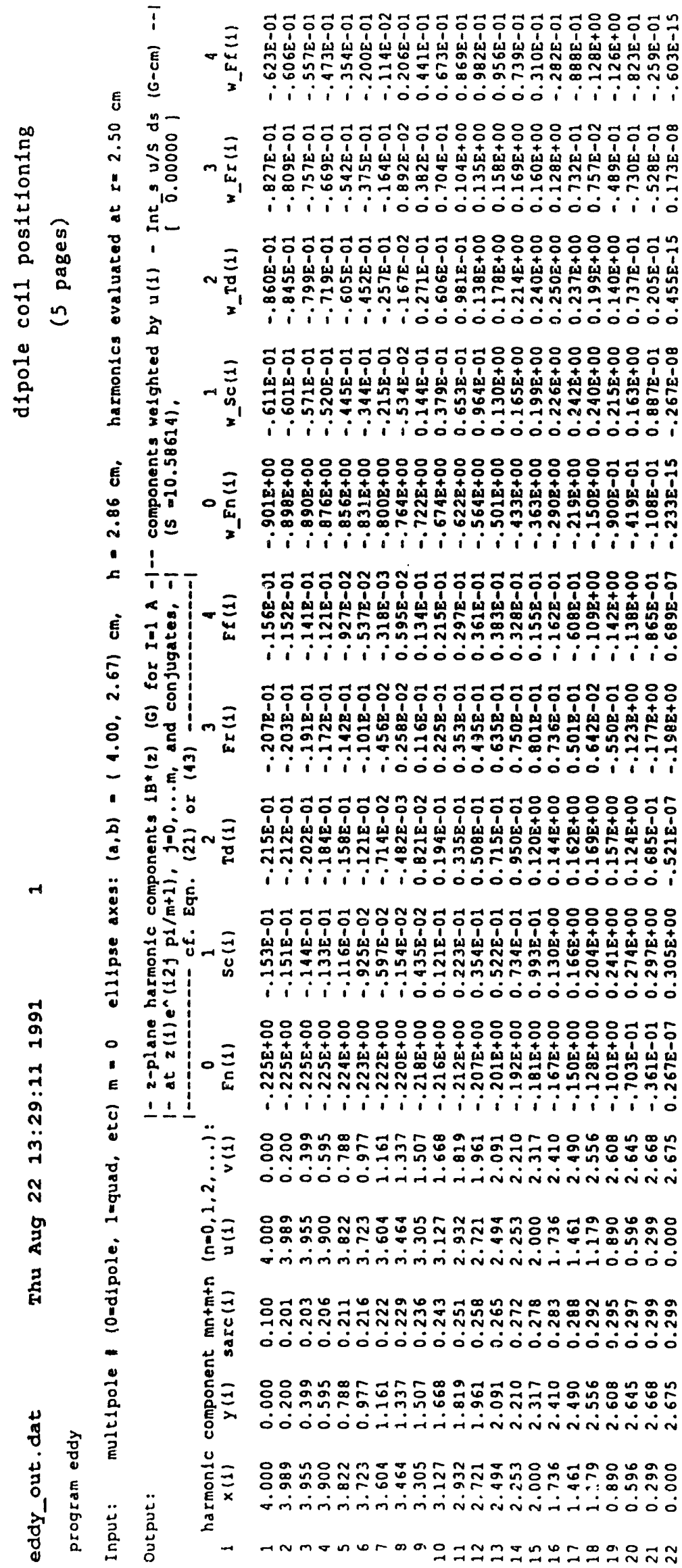

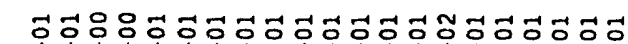

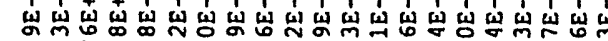

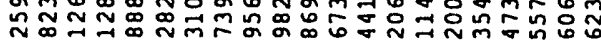

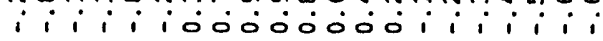

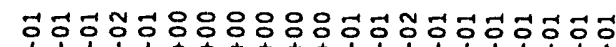

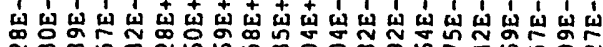
तิ oodi i i i i i i i iojóo.o

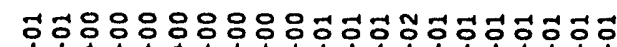

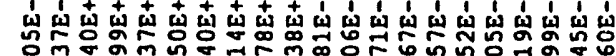

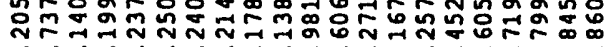
oodododododoilitili

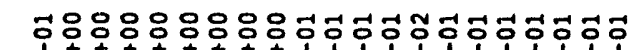

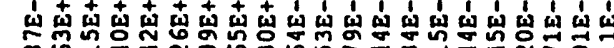

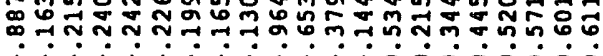
i i i i i i i i i i iojojojó

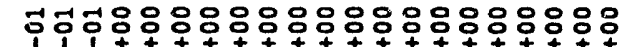

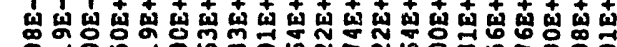

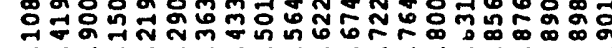
$i_{i} i_{i} i_{i} i_{i} i_{i} i_{i} i_{i}^{\infty} i_{i}^{\infty} i_{i}^{\infty}$

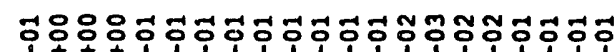

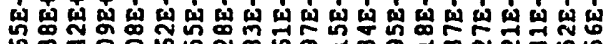

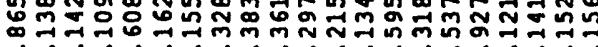

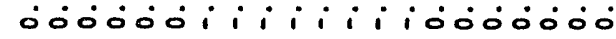

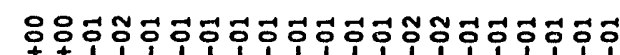

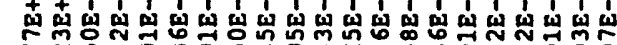

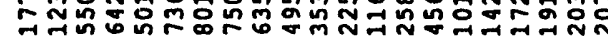
ii iojojojojojoi i i i i i

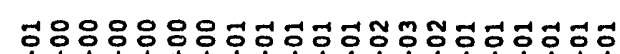

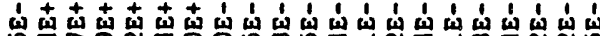

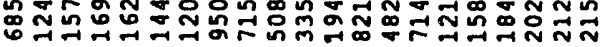
i i i i i i i i i i o o000000

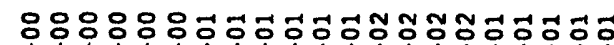

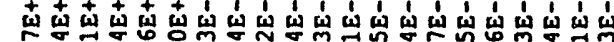

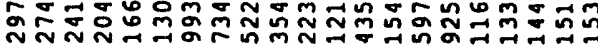
ododojojojóoili i i i i

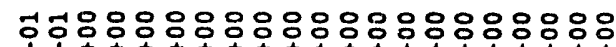

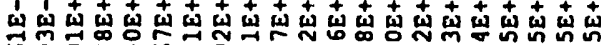

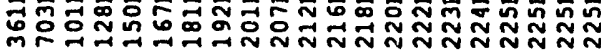
0000000000000000000

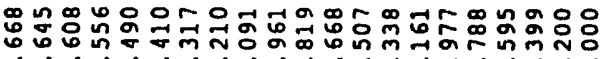

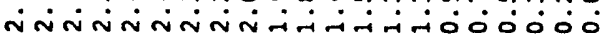

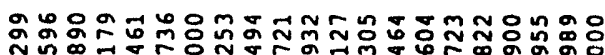

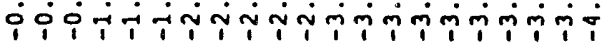

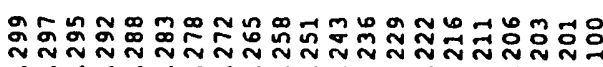

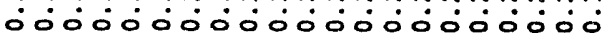

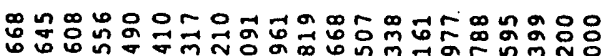

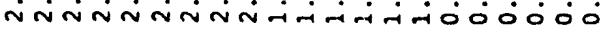

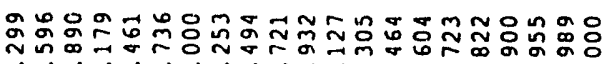

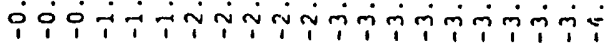

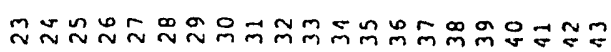




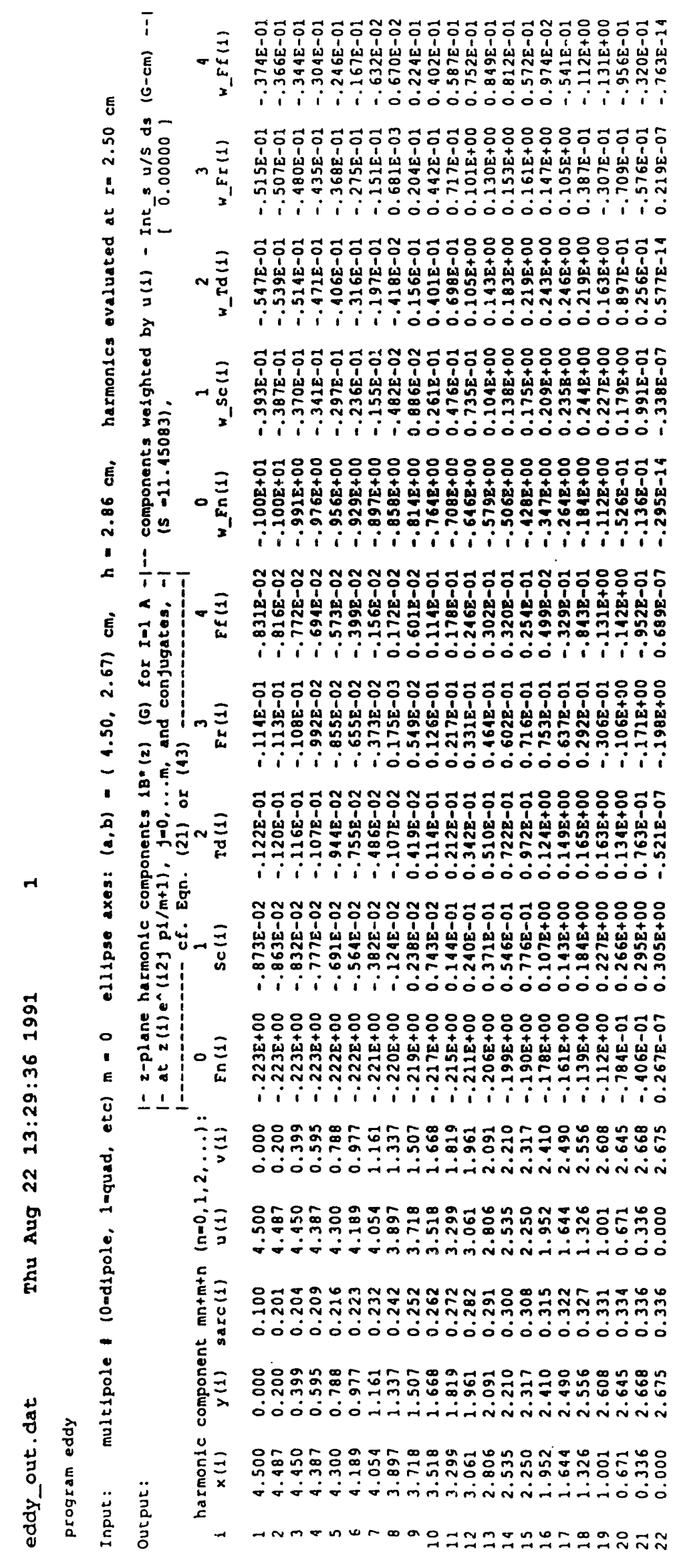

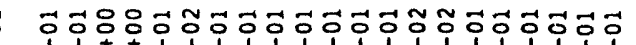

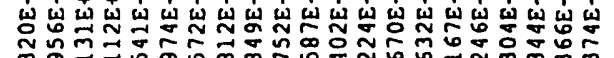

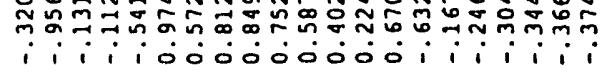

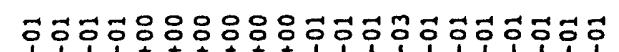

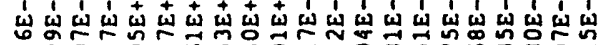

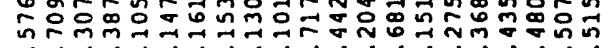
oooii i i i i i i iojojojo

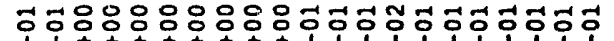

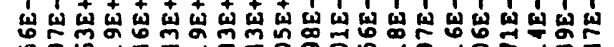

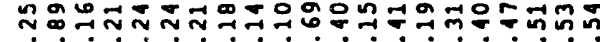
ojoojojoojoojiiliilii

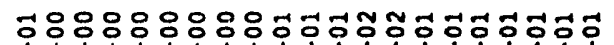

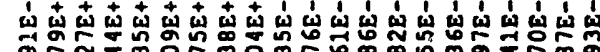

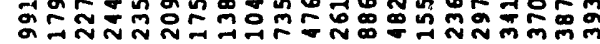
i i i i i i i i i i jojoojoo

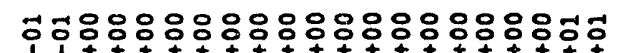

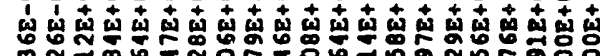

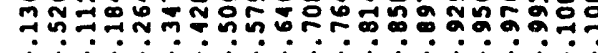

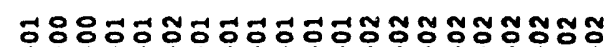

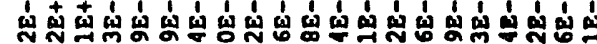

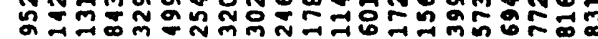
oojodi i i i i i i iojojojo

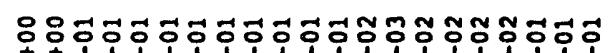

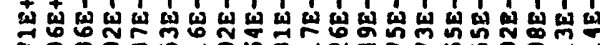

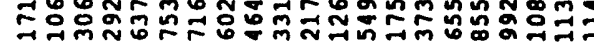
i i i00000000000i i i i i i

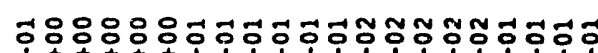

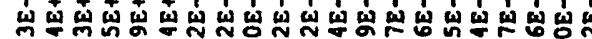

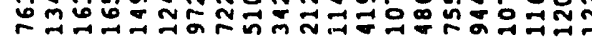
i i i i i i i i i iojojoojo

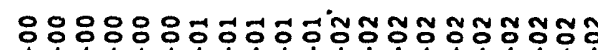

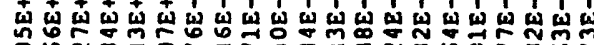

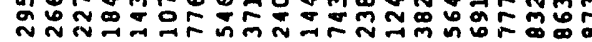
ojoojojoojoojiliilii

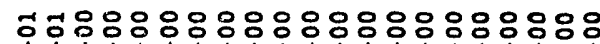

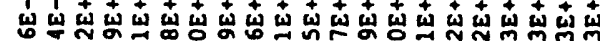

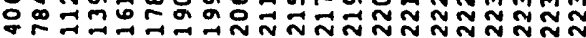
ó0000000000000000

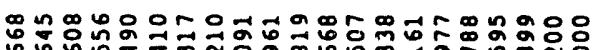

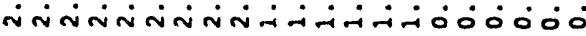

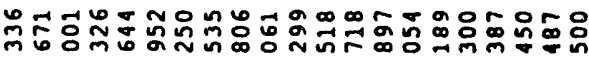

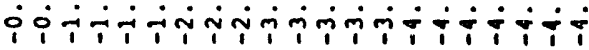

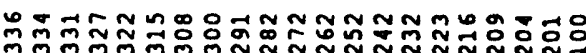
-0000000000000000000

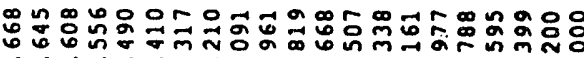

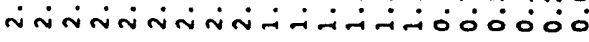

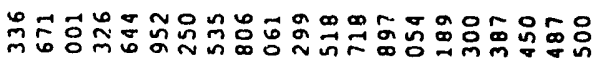

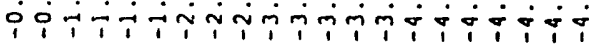

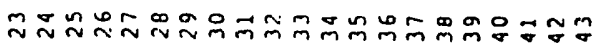




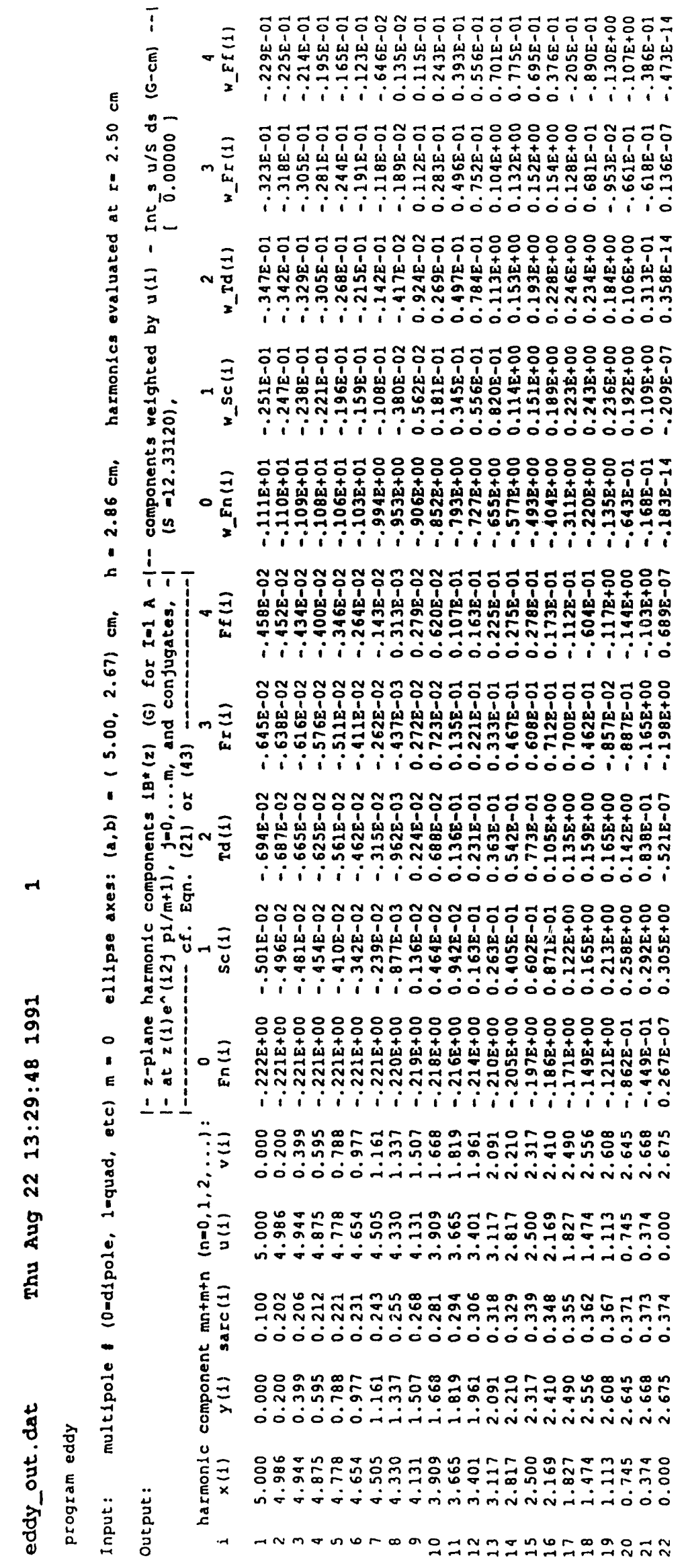

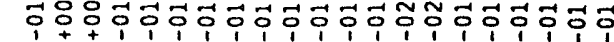

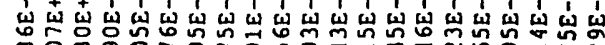

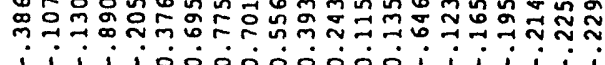

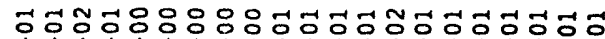

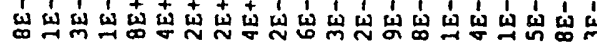

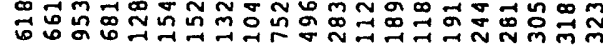

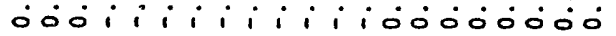

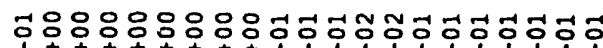

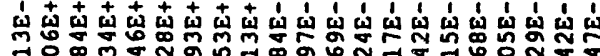

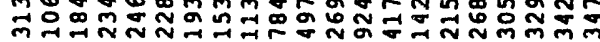
oojojoojoojooili i i i i

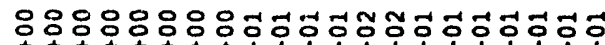

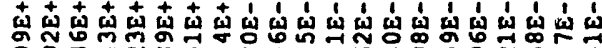

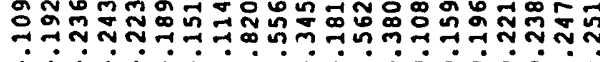
ii i i i i i i i i jojojooj

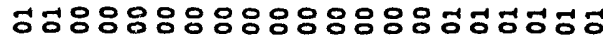

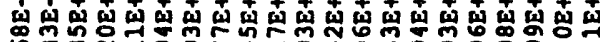

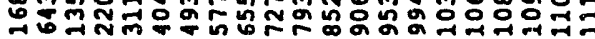
$i i_{i} i i_{i} i i_{i} i i_{i} i i_{i}$

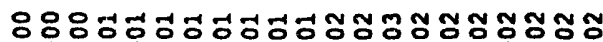

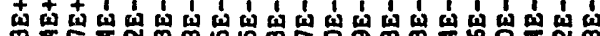

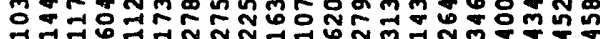
oojooi ilitiliiojojojo

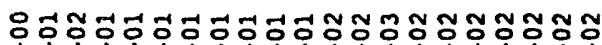
車

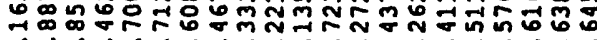

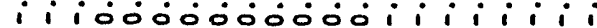

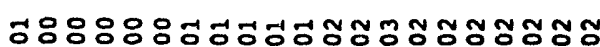

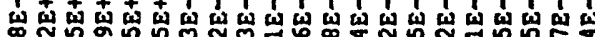

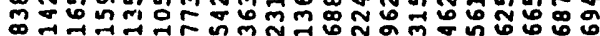
i i i i i i i i i i oojodóo

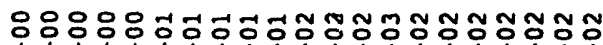

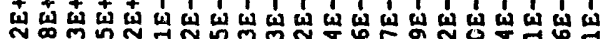

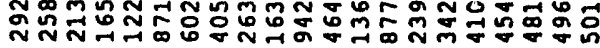
óojóojojojooi i i i i i i 겅ㅇㅇㅇㅇㅇㅇㅇㅇㅇㅇㅇㅇㅇㅇㅇㅇㅇㅇ

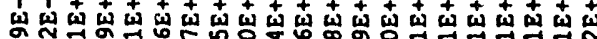

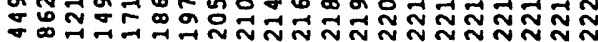
000000000000000000000

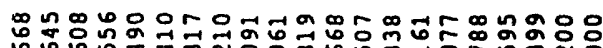

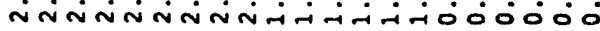

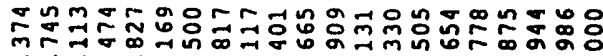

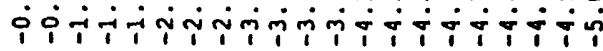

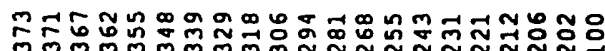
000000000000000000000

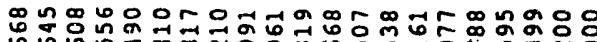

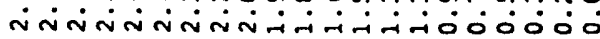

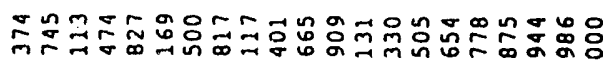

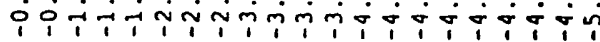

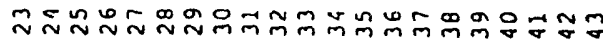




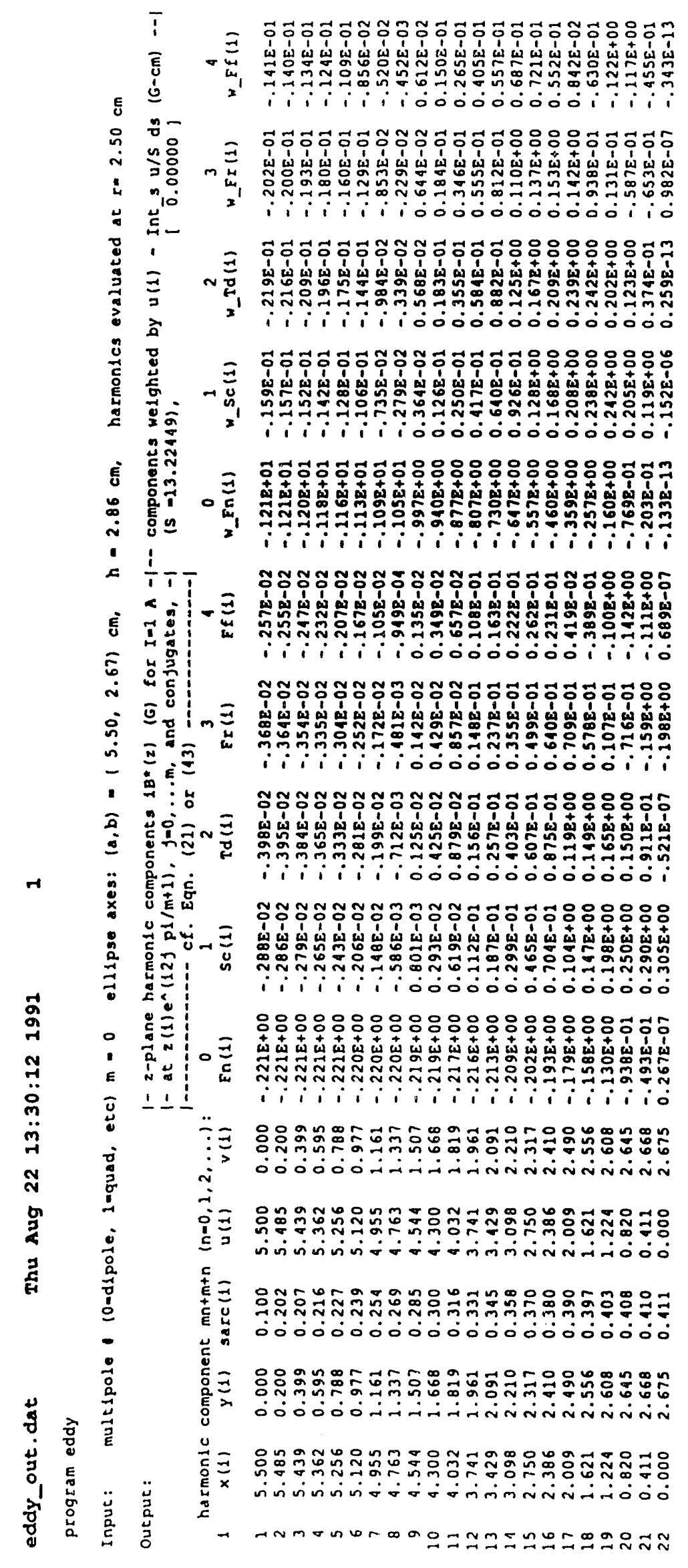

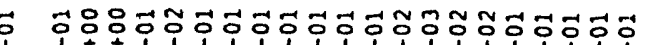

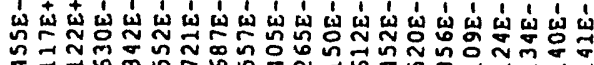
$i i$ iooosoojoi i i i

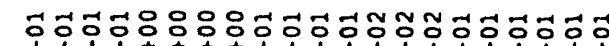

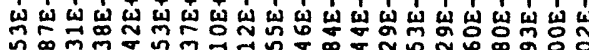
气ึ心 ooi i i i i i i iojoojo 00

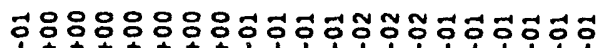

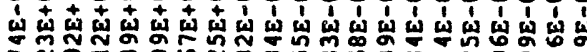

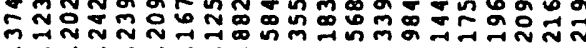
o000000000000ii i i i i

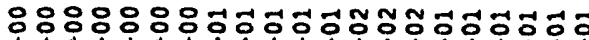

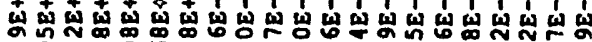

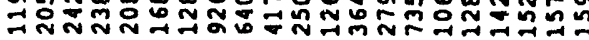
i i i i i i i i ioojo o.

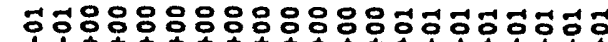

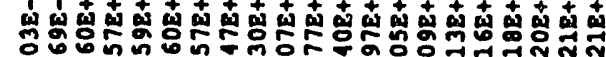

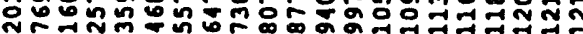
i i i i i i i i $i_{i} i$

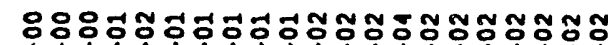

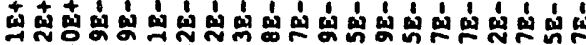

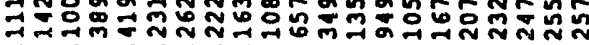

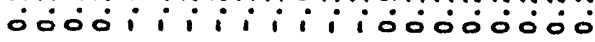

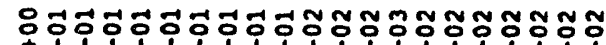

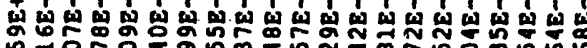

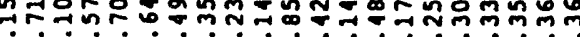
1 $1000000000001 i 1 i i i$

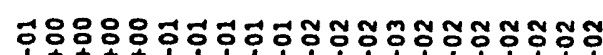

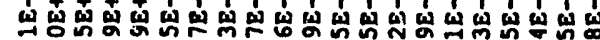

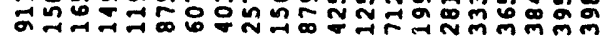
$i i_{i} i i_{i} i i_{0} 000000$

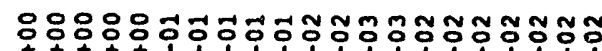

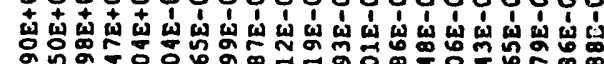

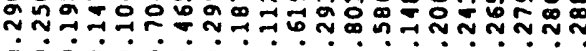
o000000000000ii ii ii

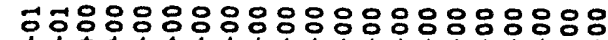

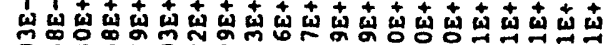

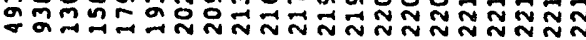
-000000000000000000

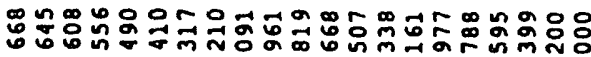

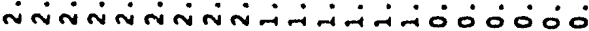

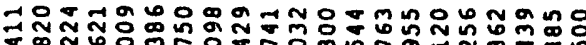

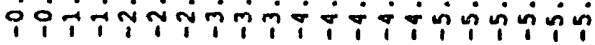

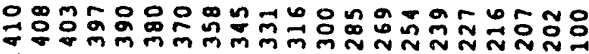
000000000000000000000

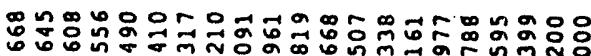

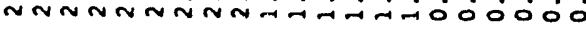

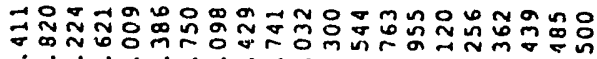

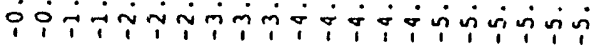
๙ 


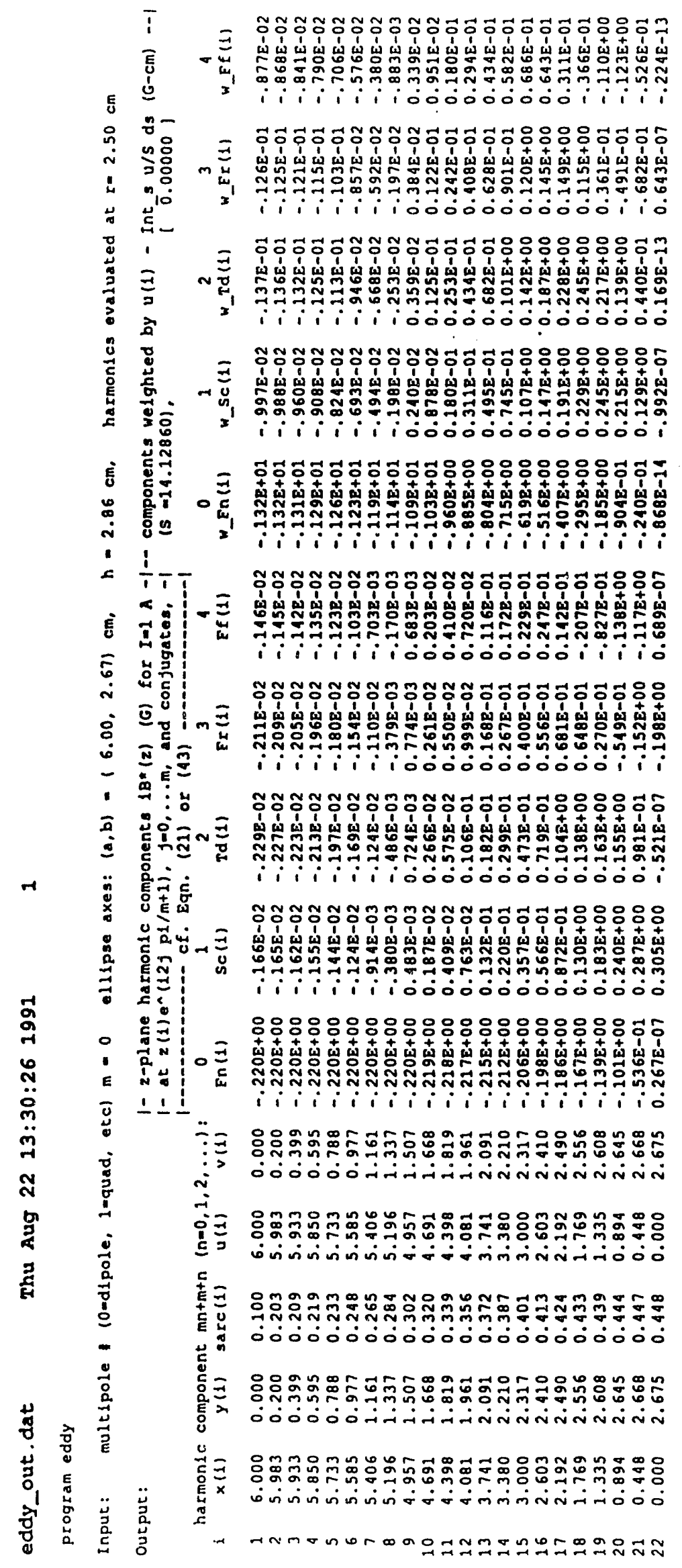

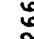

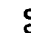

\section{I}

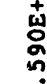$$
\circ
$$

\section{安

\section{$\ddot{4}$}

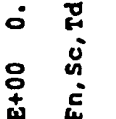

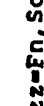

\section{景}

\section{ì}

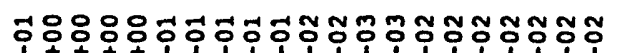




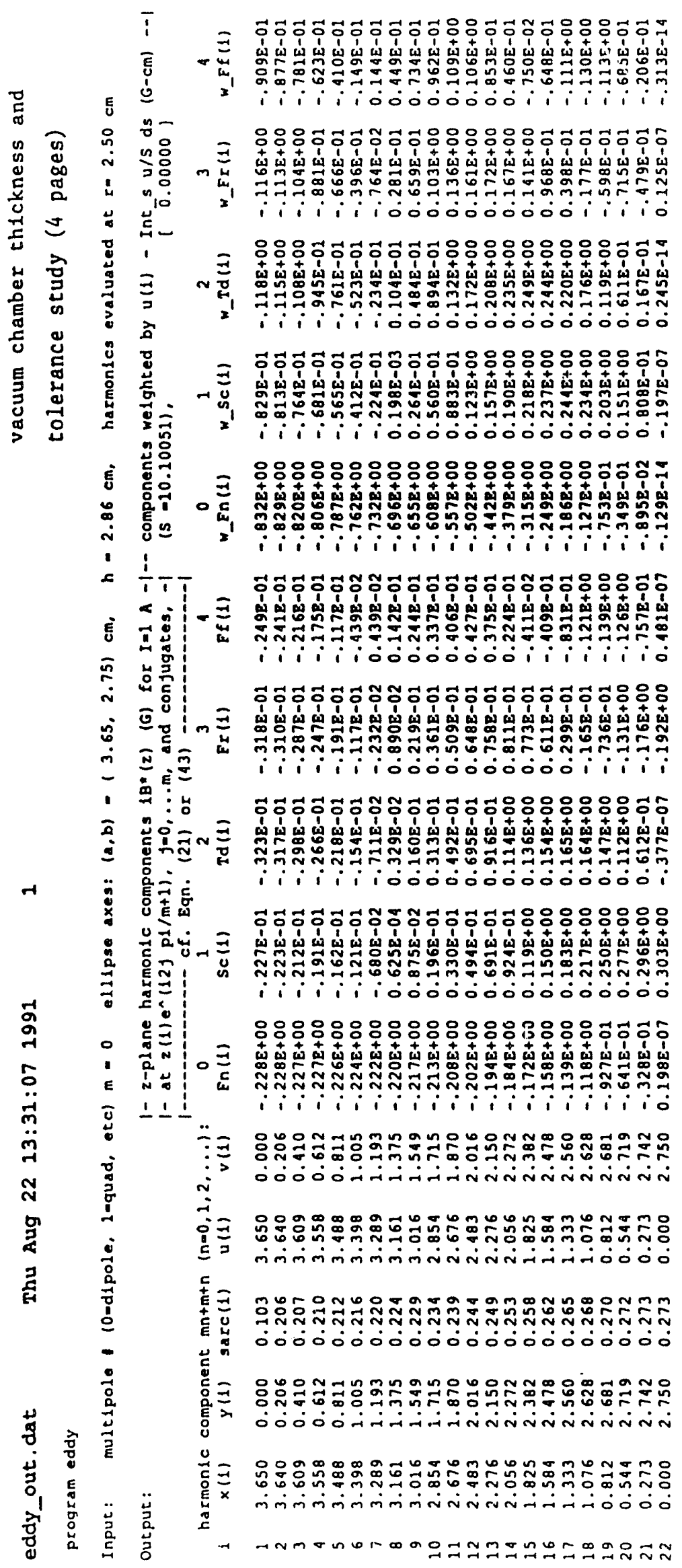

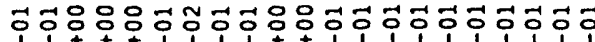

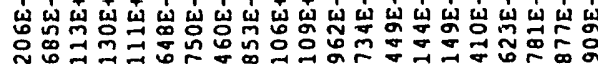

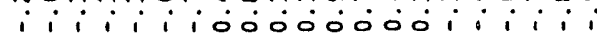

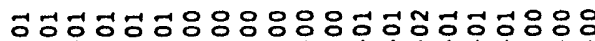

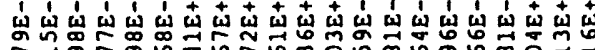
б oojoiiiiiiiiiiojojojo

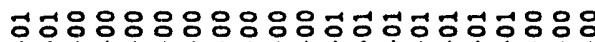

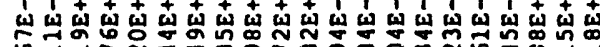

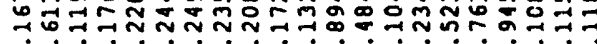
o0o0o000000000i i i i i

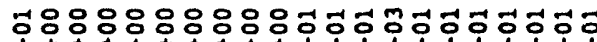

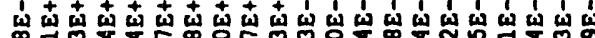

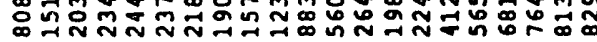
ii i i i i i i i i iojojooo

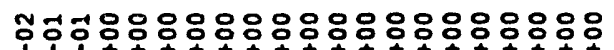

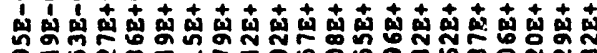

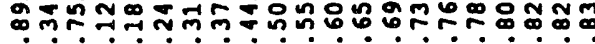
i i i i i i i i i i i i i i

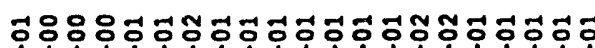
㟧

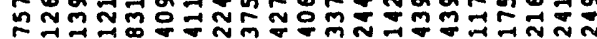
ojoojodiliiiiiiojojoo

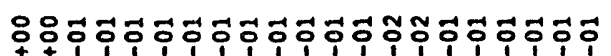

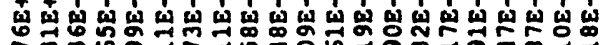

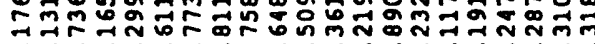
i i i iojodoojoodi i i i i

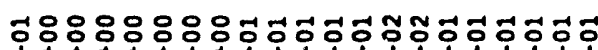

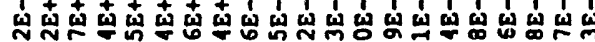

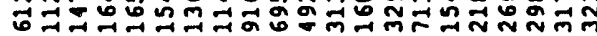
$i_{i} i_{i} i_{i} i_{i} i_{i} i_{i}$ iojojojo

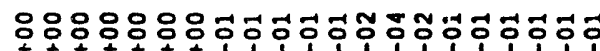

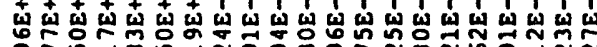

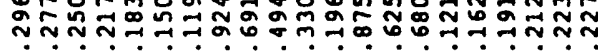
o0000000000000ili i i

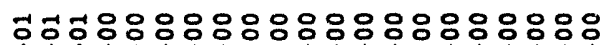

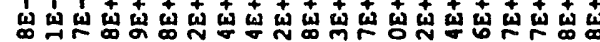

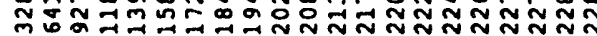
00000000000000000000

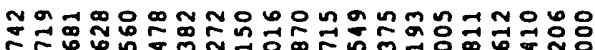

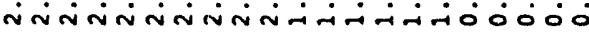

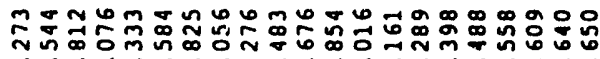

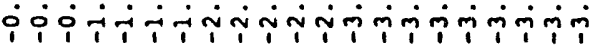

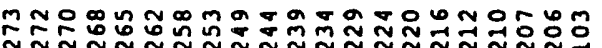
o00000000000000000000

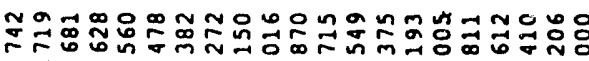

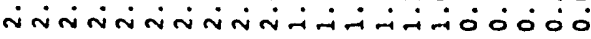

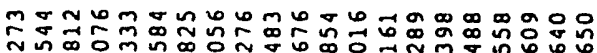

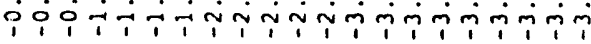

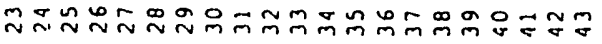




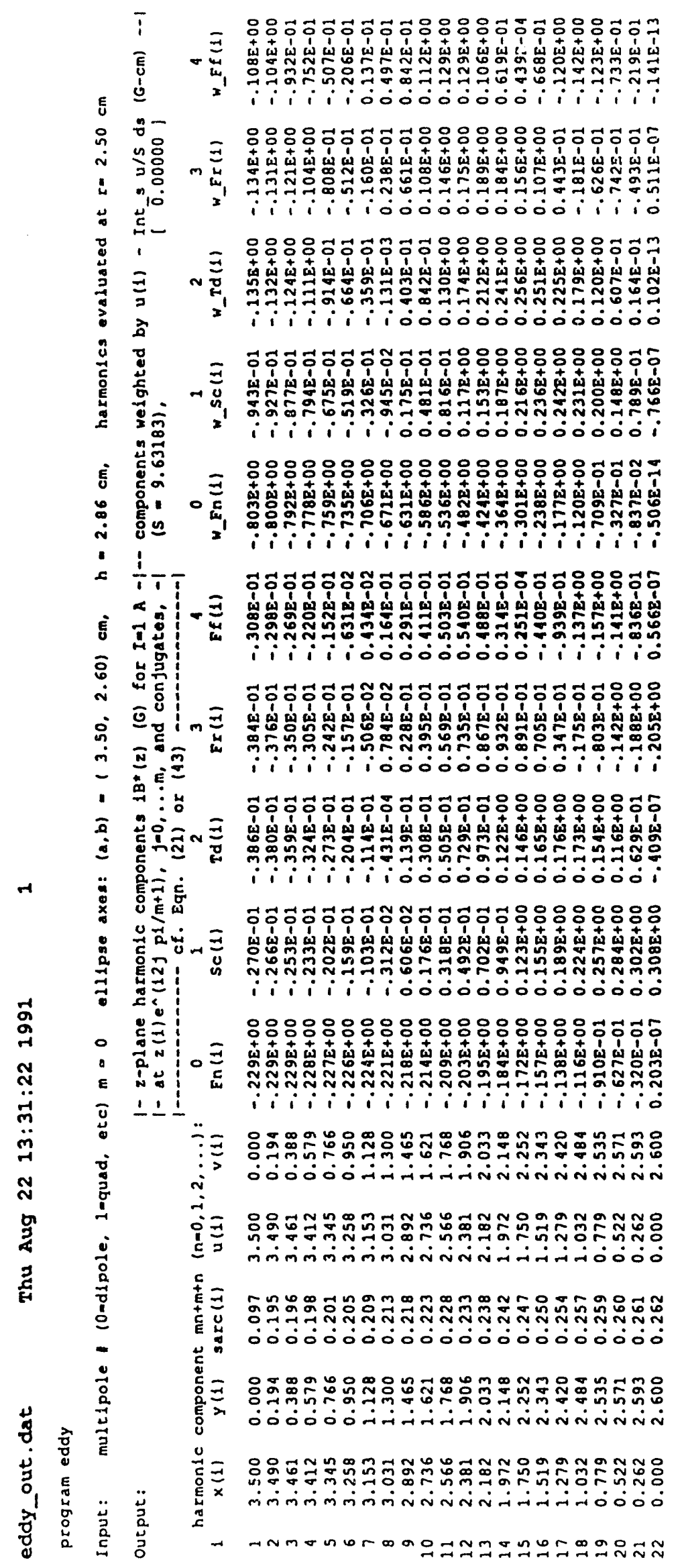

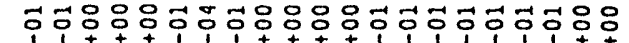

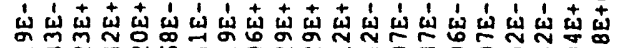
กิ ii i i iojojoojojii i i i

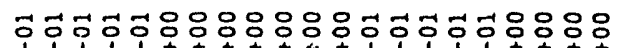

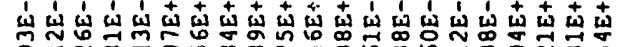

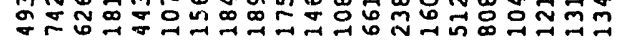
oojoiiiiiiiiiiojojóo

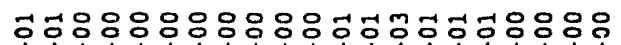

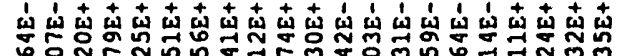

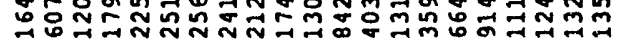
00000000000i $i_{i} i_{i} i$

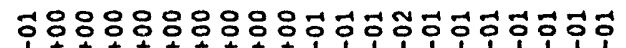

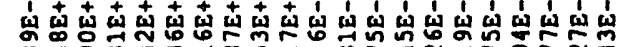

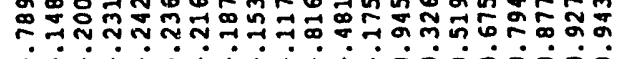
$i_{i} i_{i} i i_{i} i i_{0}$ jojojó

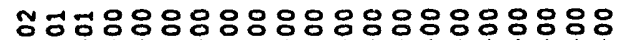

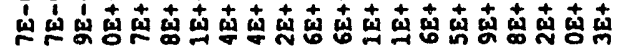

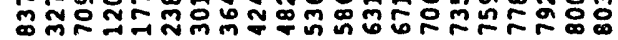
i i i i i i i i i i i i

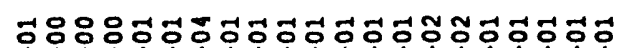

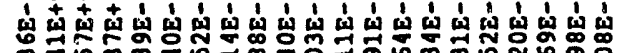

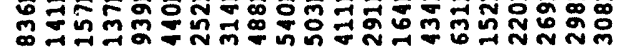

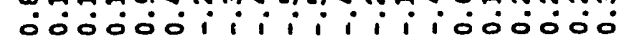

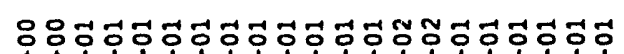

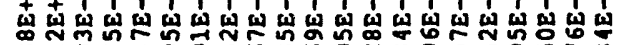

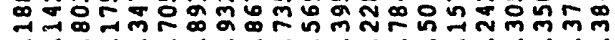
iiiiojojo00000iiliii

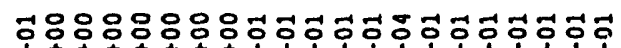

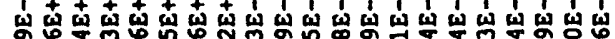

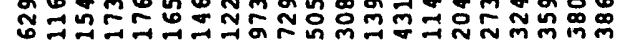
$i i_{i} i i_{i} i i_{1} 0000000$

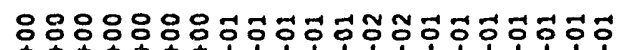

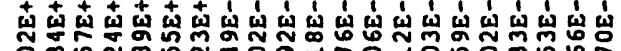

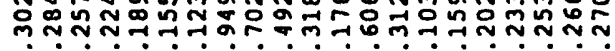
0000000000000i1iiiiii

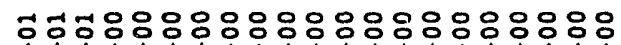

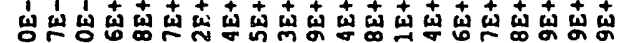

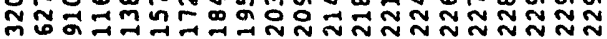
000000000000000000000

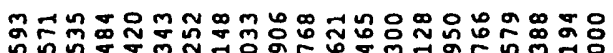

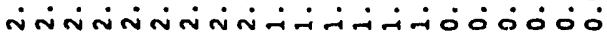

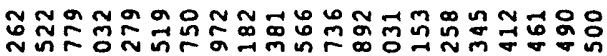

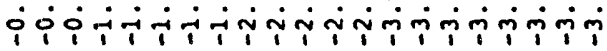

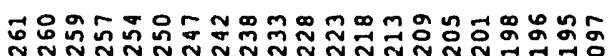
000000000000000000000

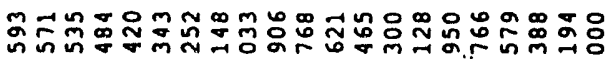

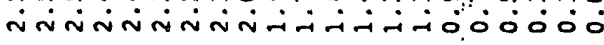

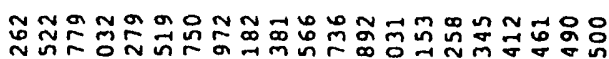

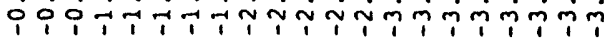

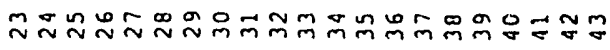
(n)

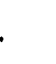




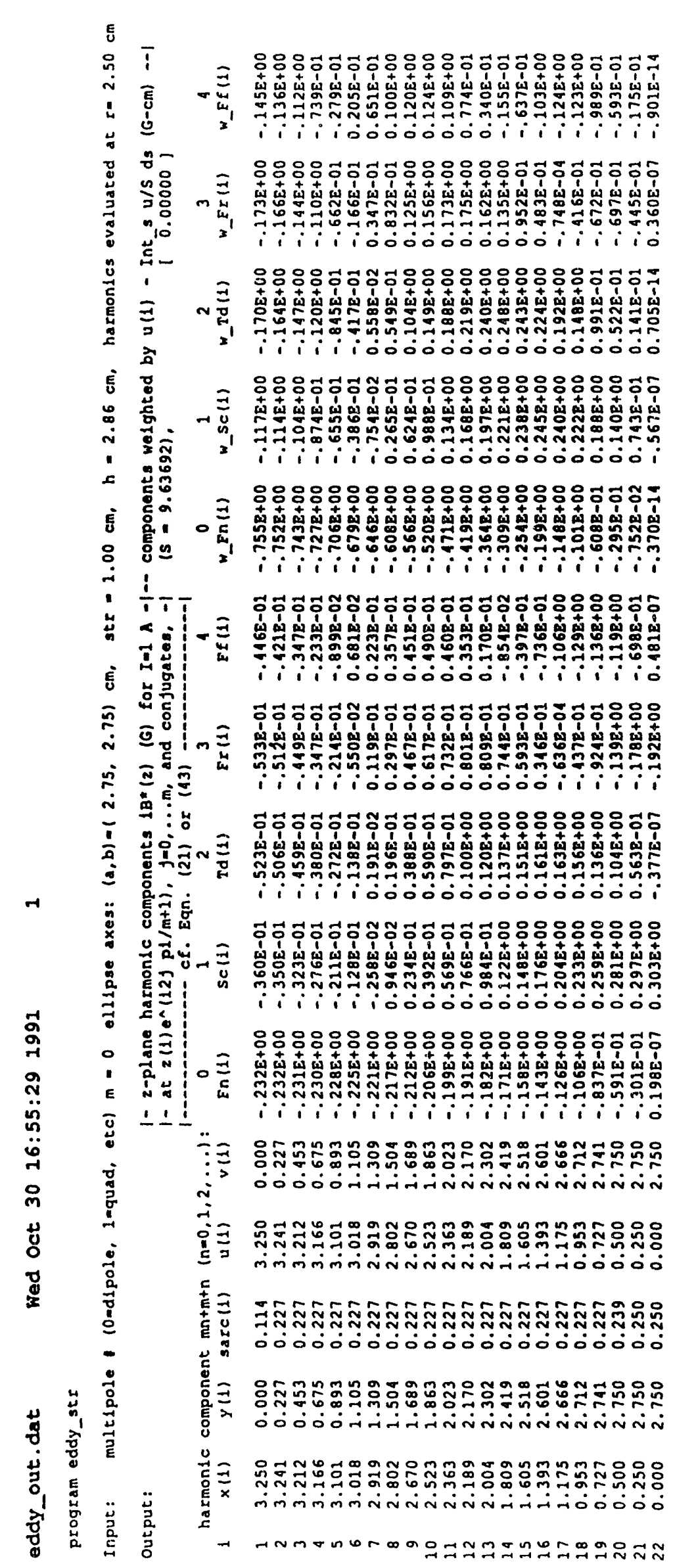

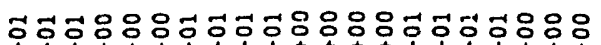

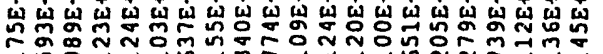

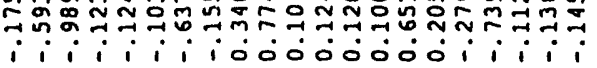

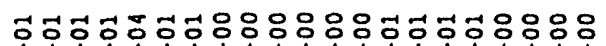

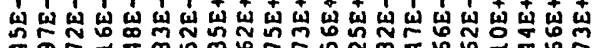

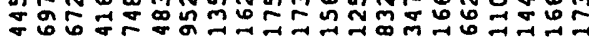

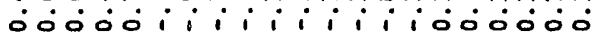

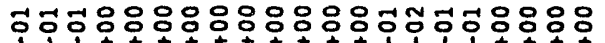

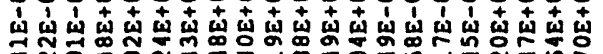

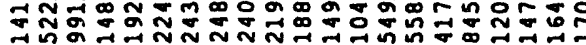
S00000000000000i i i i i

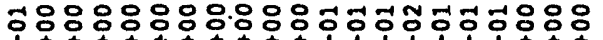

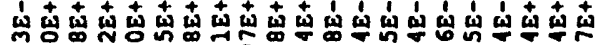

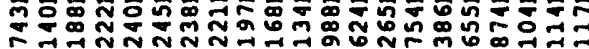
i i.iiiiiiiiio00000

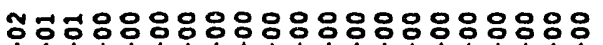

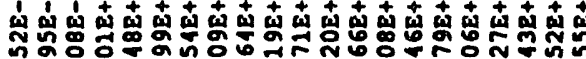
nू. ii i i i i i i i i i i i

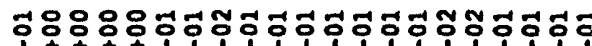

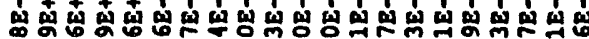

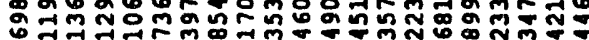

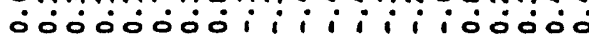

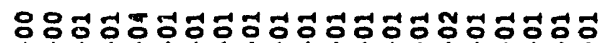

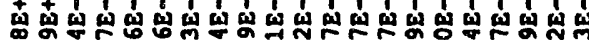

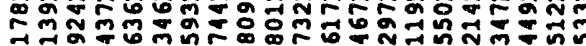
$i i i i$ i $000000000 i i i_{i}$

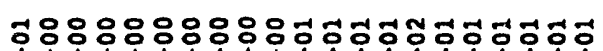

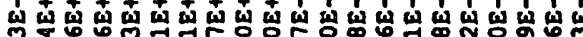

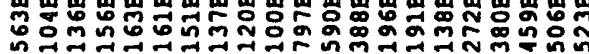
$i_{i} i i_{i} i i_{i} i_{i 00} 000$

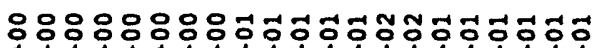

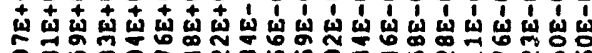

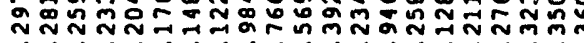
o0000000000000iiiiii

근ㅎㄷㅇㅇㅇㅇㅇㅇㅇㅇㅇㅇㅇㅇㅇㅇㅇㅇㅇㅇㅇ

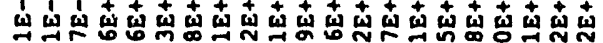

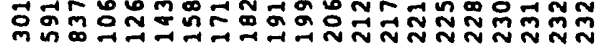
o0ं0000000000000000

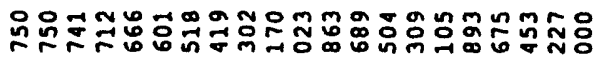

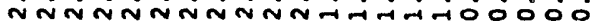

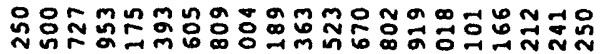

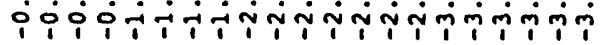

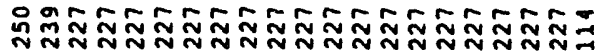
00000000000000000000

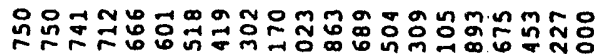

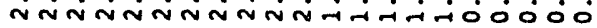

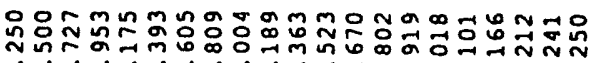

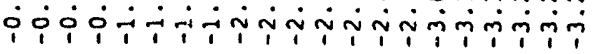

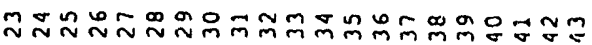




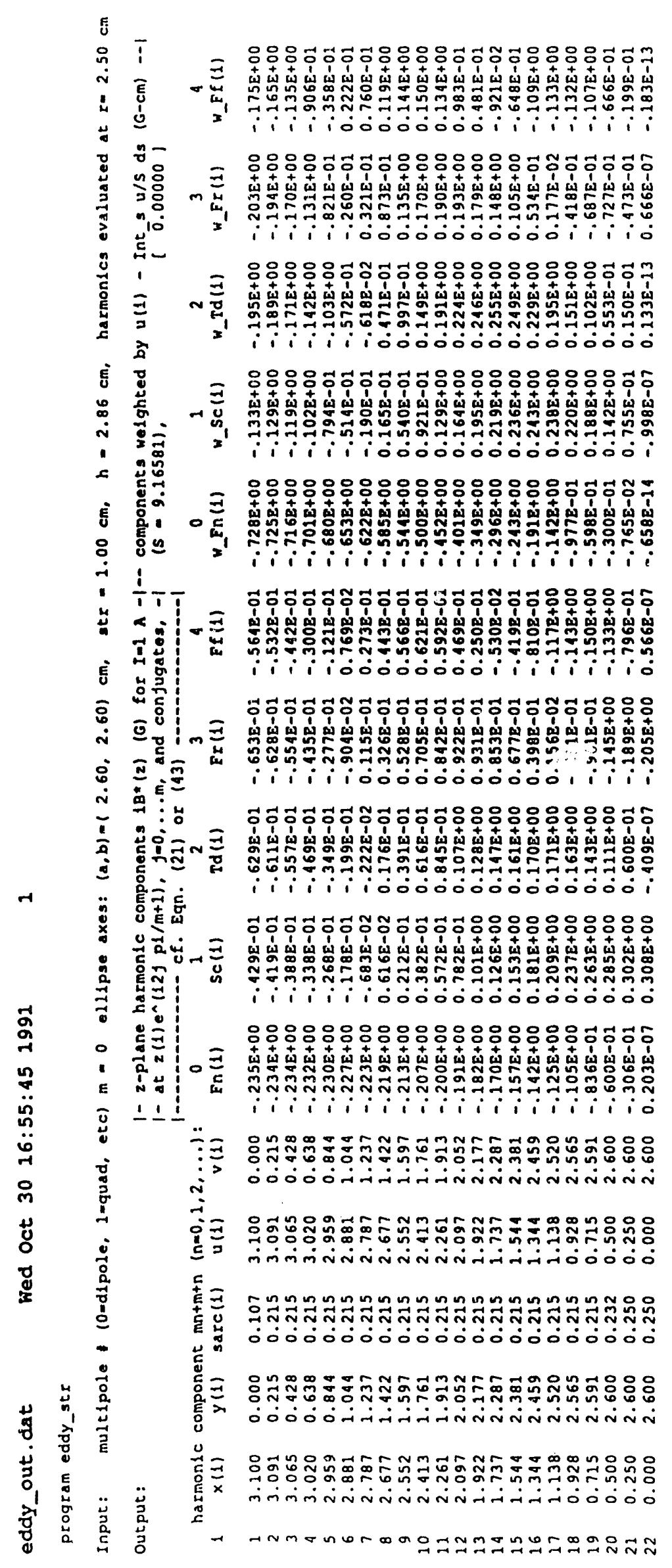

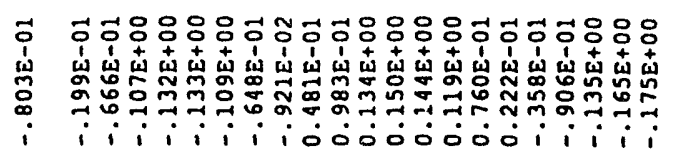

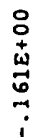

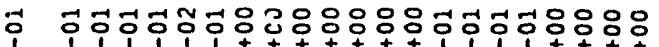

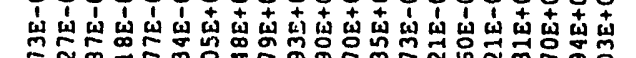

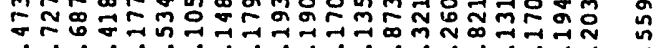
oojoiiiiiiiiiiojojoo

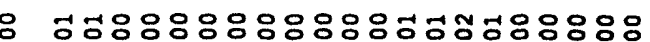

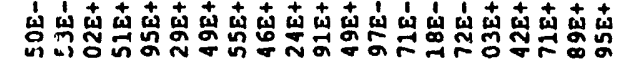

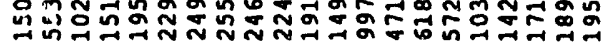
oojojojodoodioi i i i i

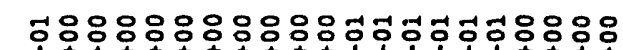

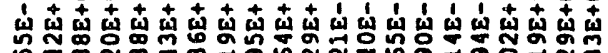

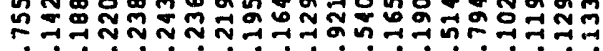
$i_{i} i_{i} i_{i} i_{i} i_{i} i_{i}$ oodojó

ก๊

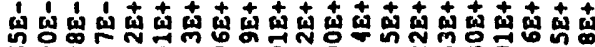

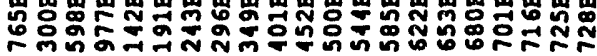
i i i i i i i i i i i i i i i i

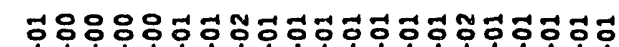

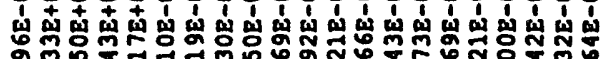

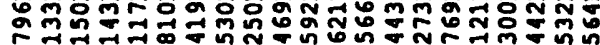

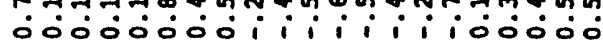

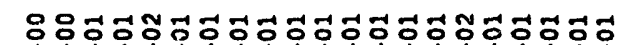

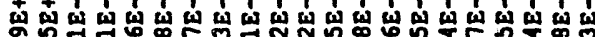

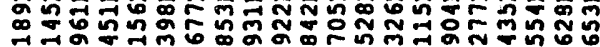
i i i iojojojojojoi i i i i

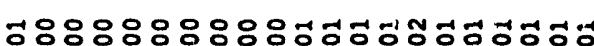

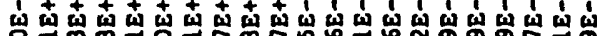

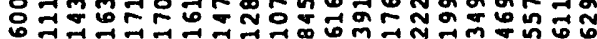
$i_{i} i_{i} i_{i} i_{i} i_{i} i_{0}$ oojojo

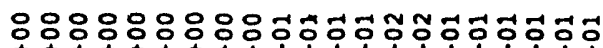

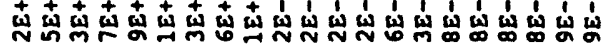

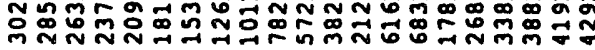
ojoojojojojodoi i i i i i

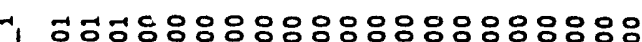

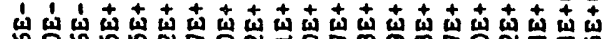

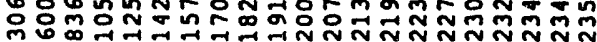
o000000000000000000

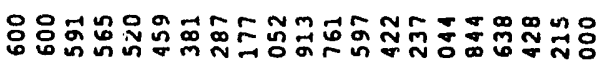

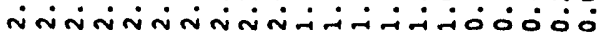

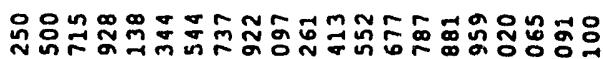

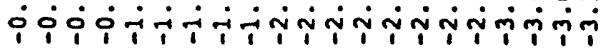

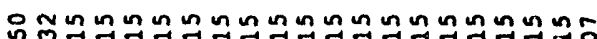

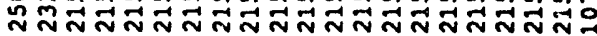
00000000000000000000

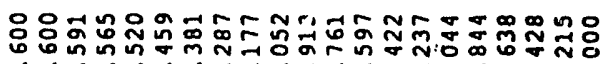

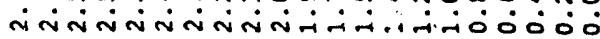

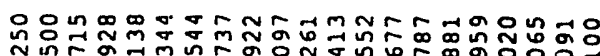

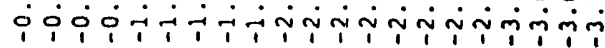

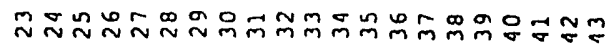




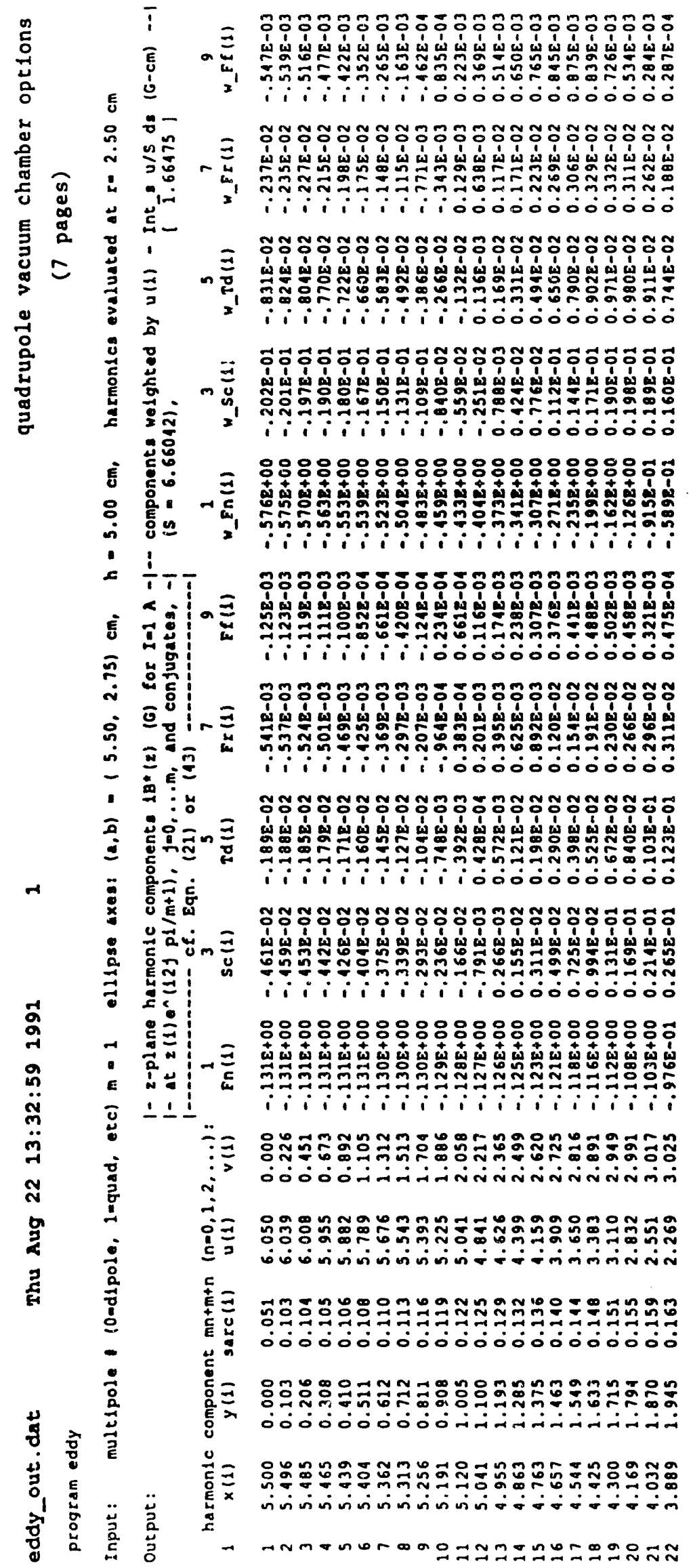

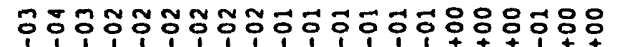

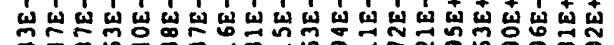

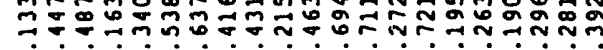

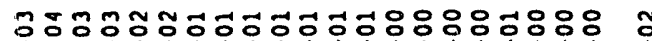

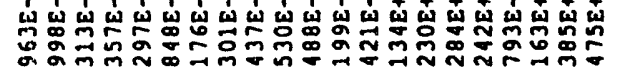

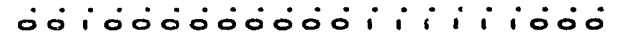

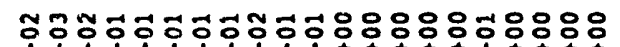

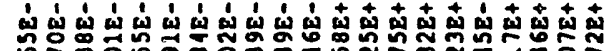

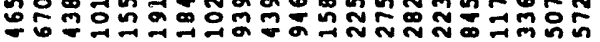
ói i i i ioosoojoosi i i

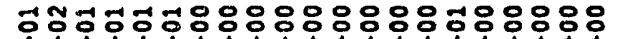

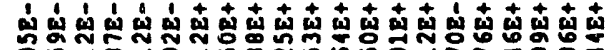

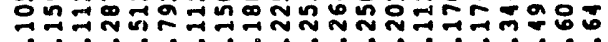
o0ii i i i i i 1000000

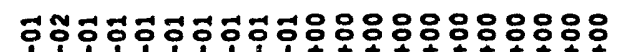

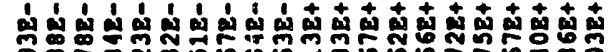

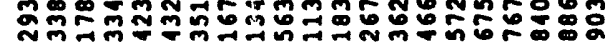

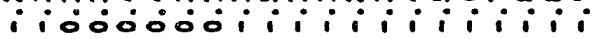

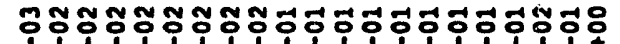

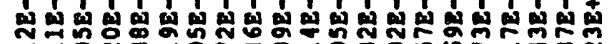

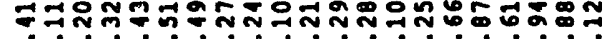
$i i i i i_{0} 00000 i i$ i000

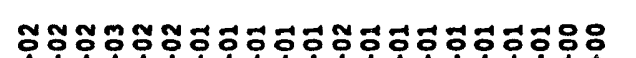

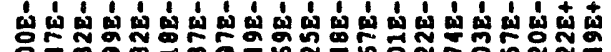

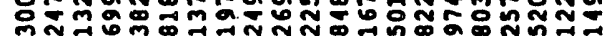

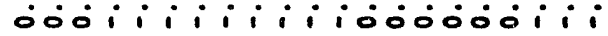

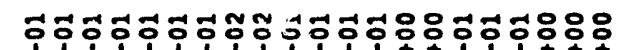

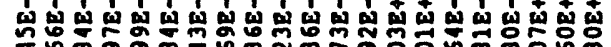
00000000i i i i í000

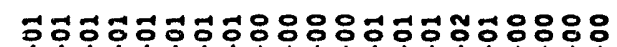

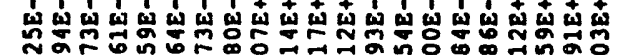
స్ల. o00000000000000ii i i

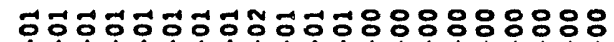

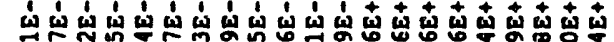

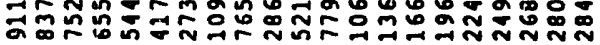
i i i ío00000000000

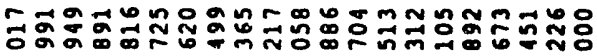

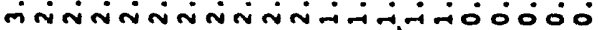

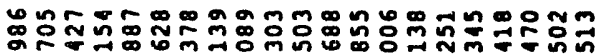

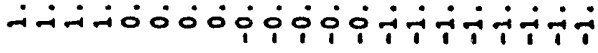

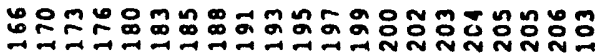

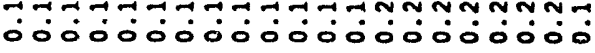

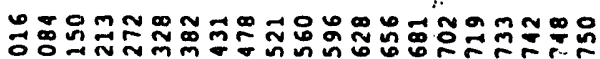

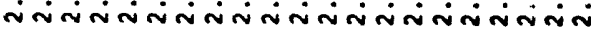

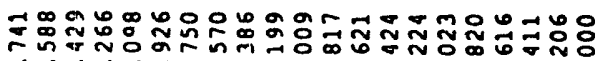

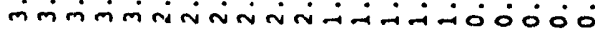

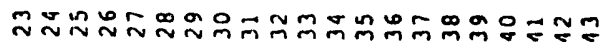




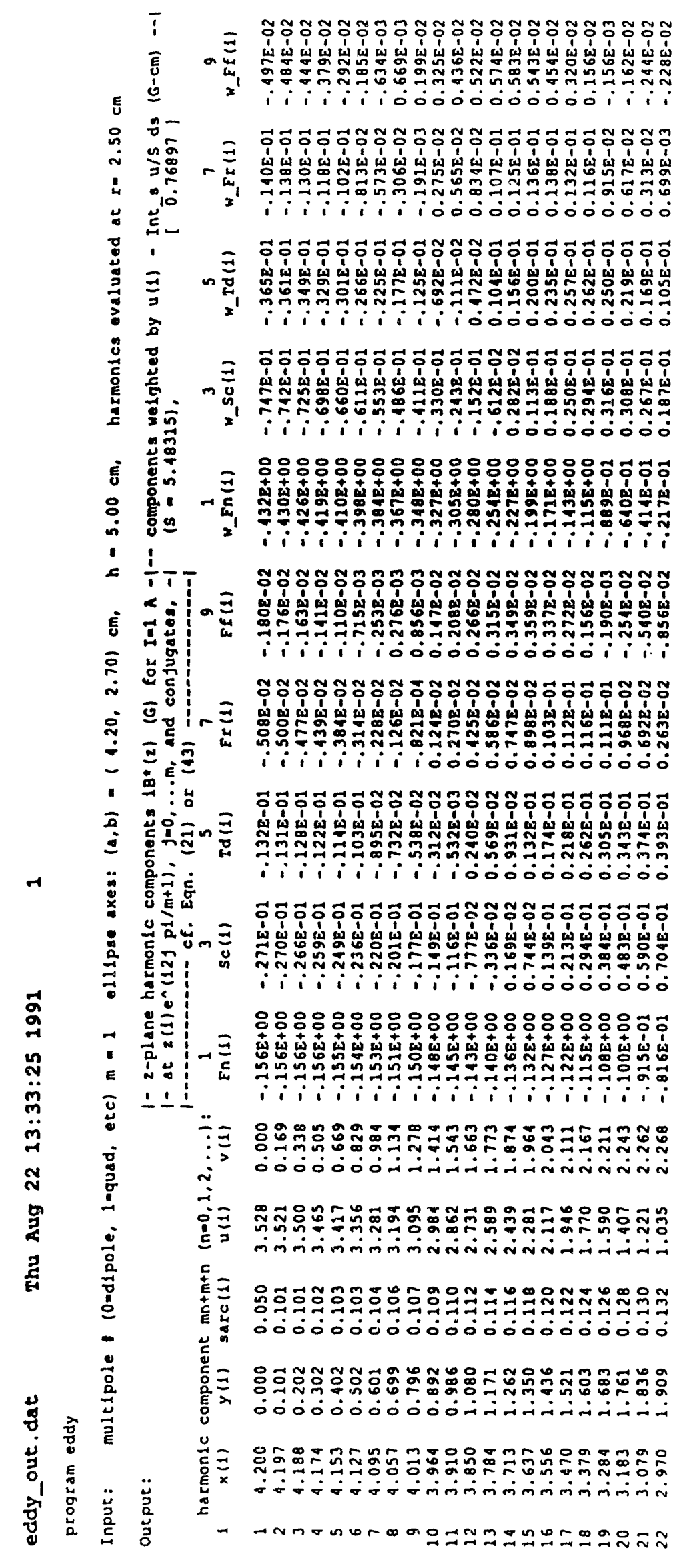

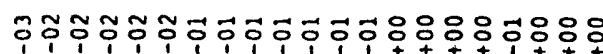

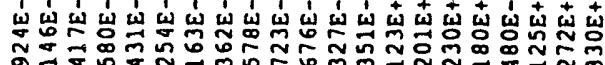
iojosiiili iojosoili

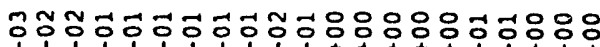

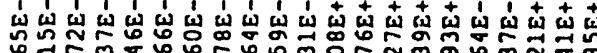

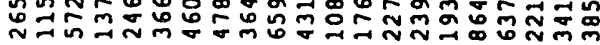
io00000000i i i i i

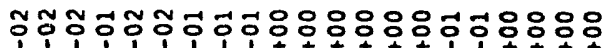

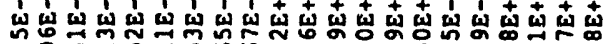

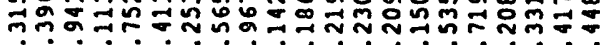
oi i ioojo-000000i i i

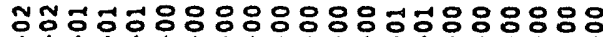

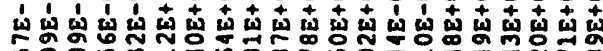

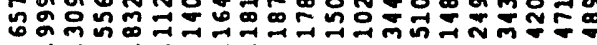
oi iiiiiiiiiio000000

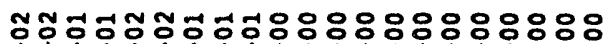

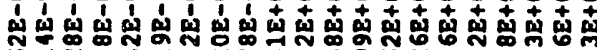

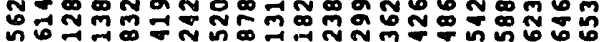
ióo: $i_{i} i_{i} i_{i} i_{i} i_{i} i_{i}$

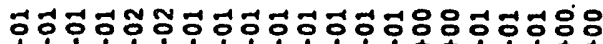

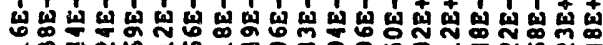

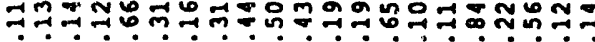

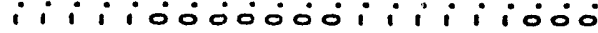

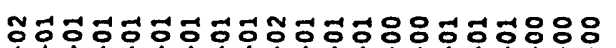

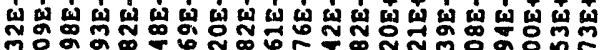
ల్m i i i i i io000000ii i

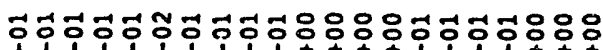

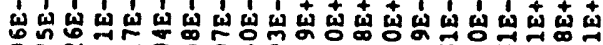

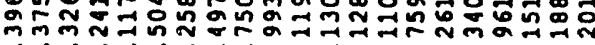

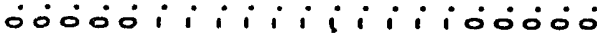

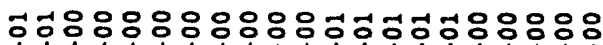

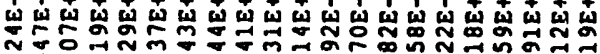

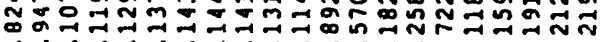
0000000000000்i i i i

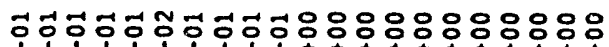

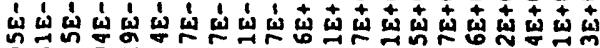

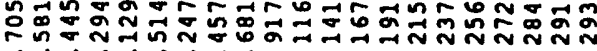

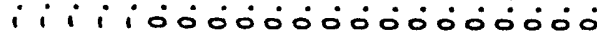

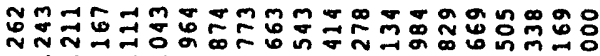

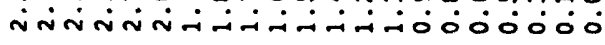

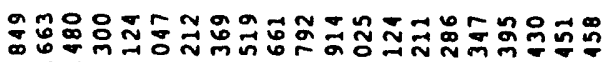
ooooipioipititititit

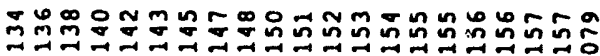
-0000000000000000

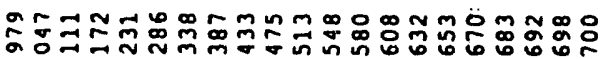

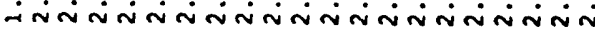

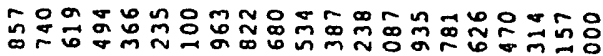

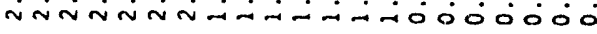

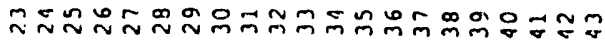




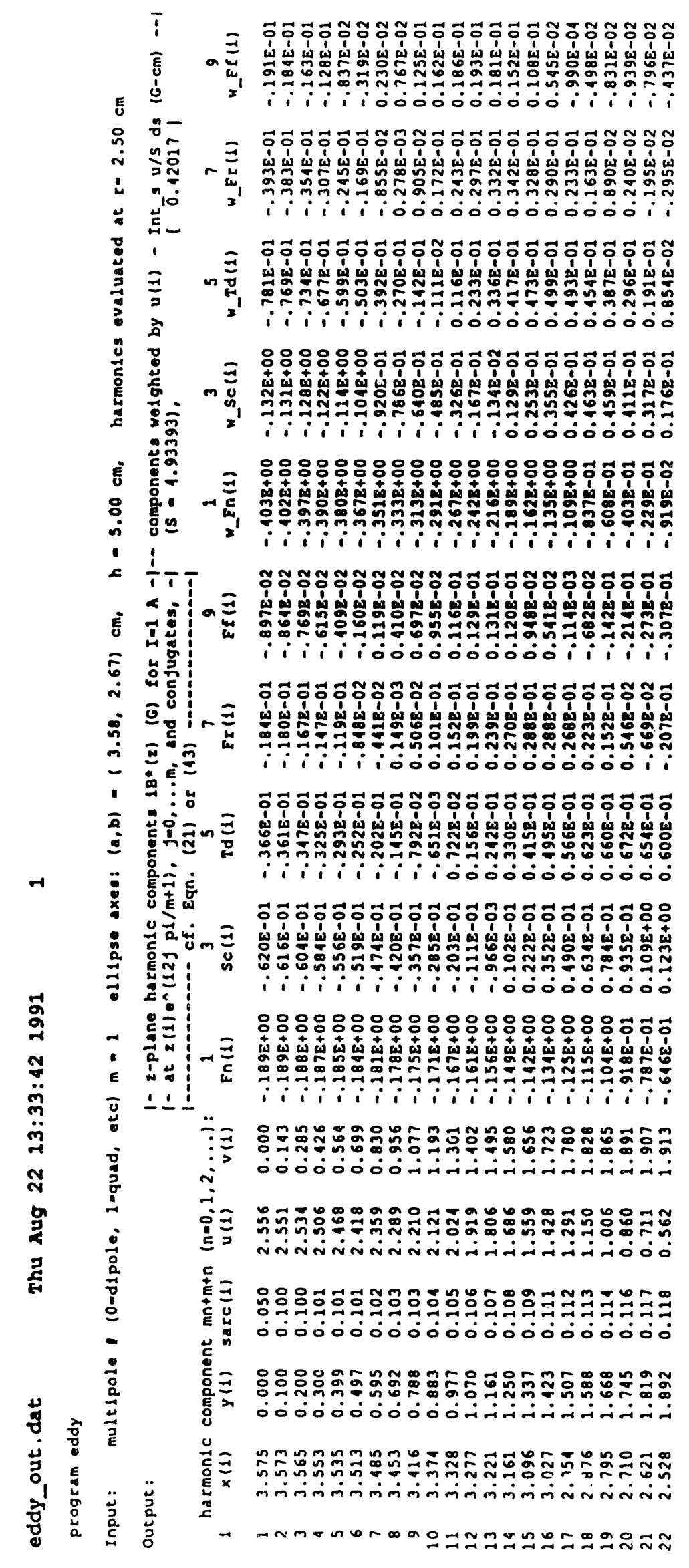

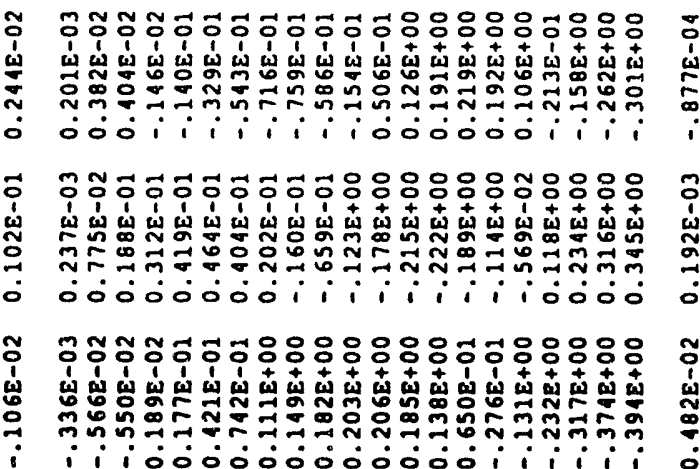

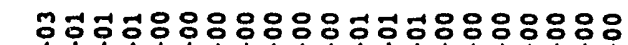

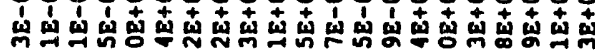

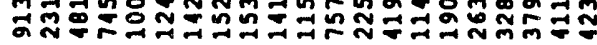

i i i i i i i :000000

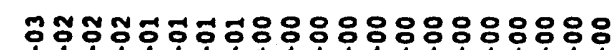

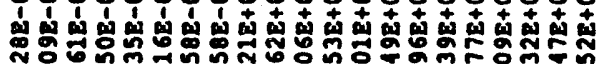

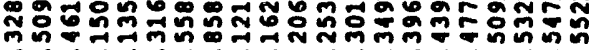

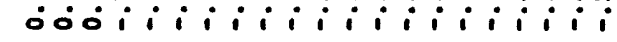

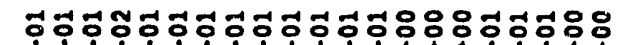

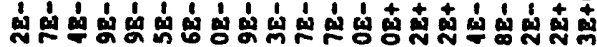

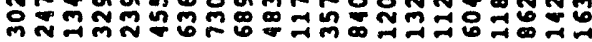
i i00000000 ili i io000

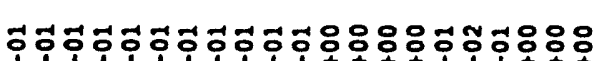

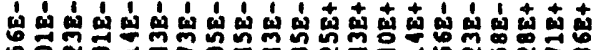

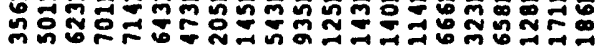
iiiiiiioo000000ii i

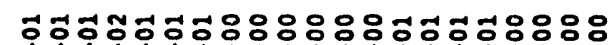

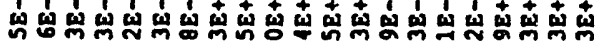

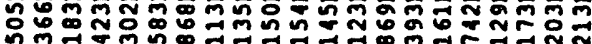
000iiiiiiilito00000

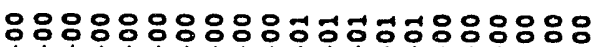

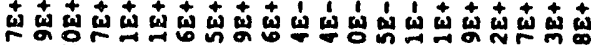

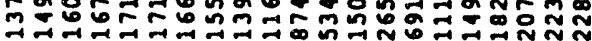
0000000000000i i i i?

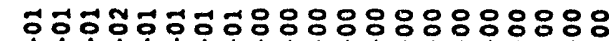

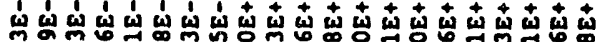

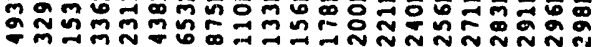
i i iojo0000000000000

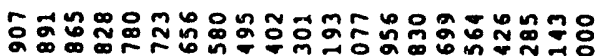

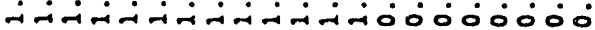

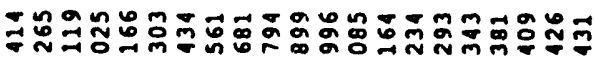

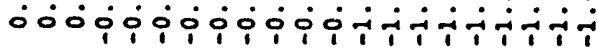

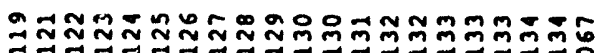
o0000000000000000

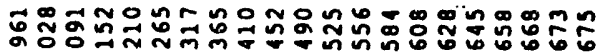

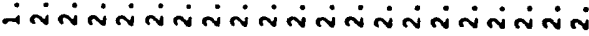

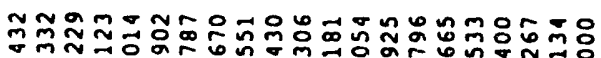

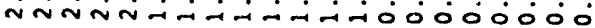

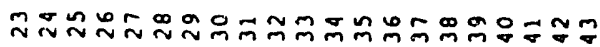




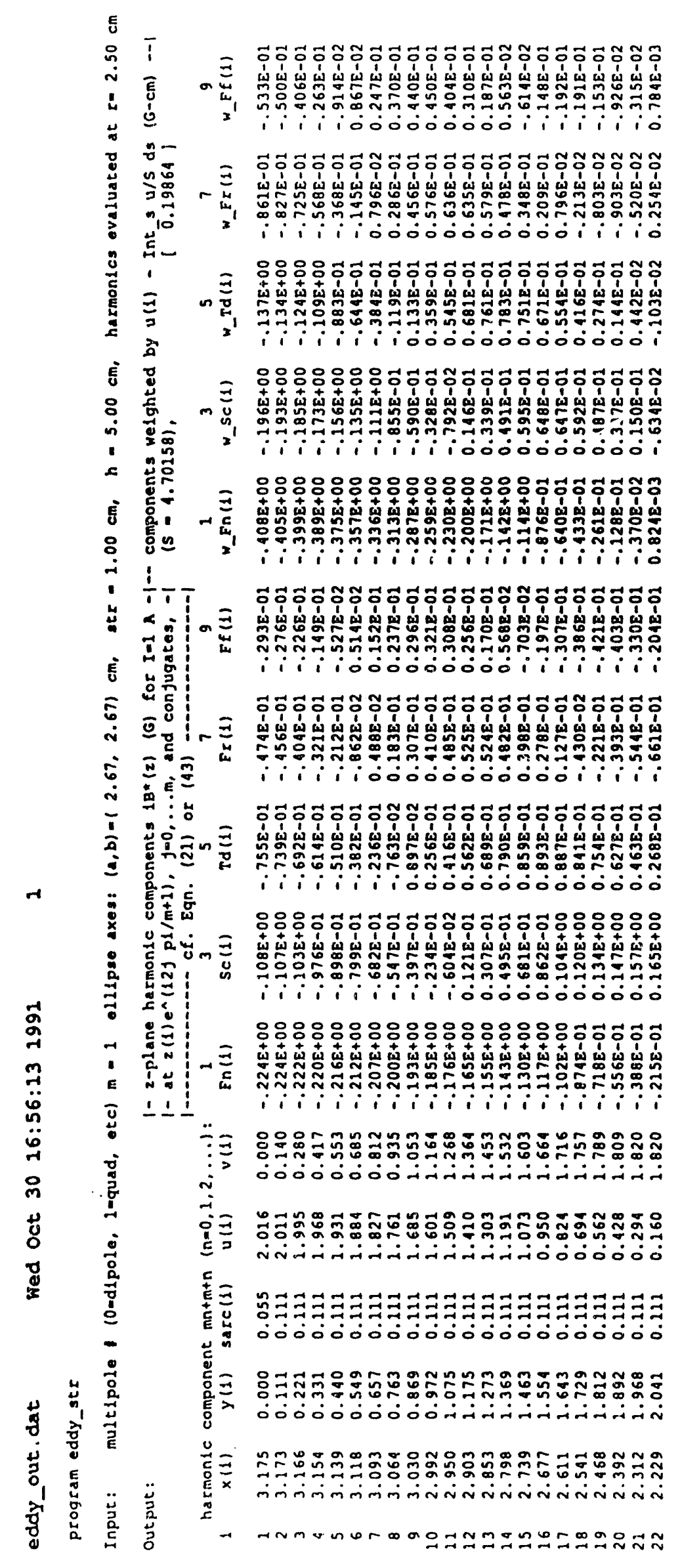

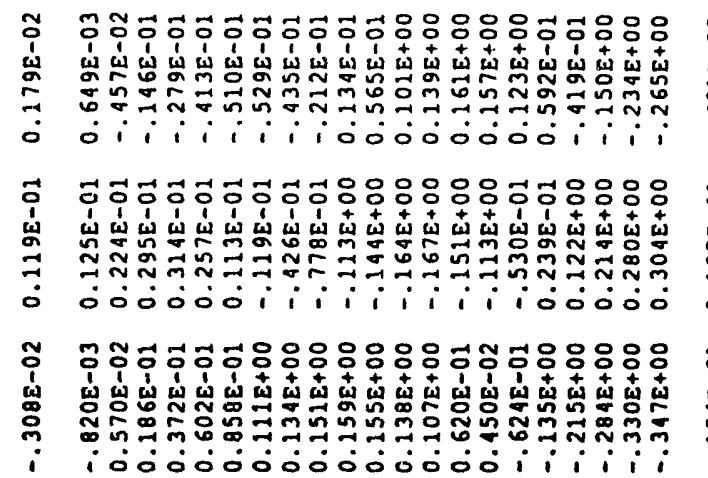
ก ลับ ô

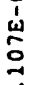

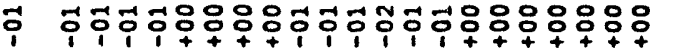

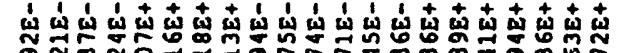

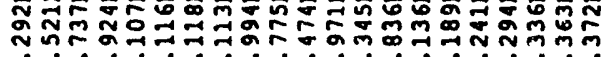
i i i i i i i ioojo0000

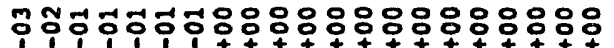

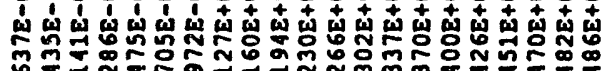
oi i i i i i i i i i i i i

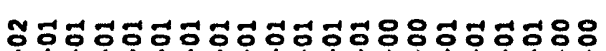

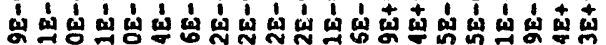

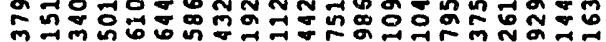
i00000000i i i i i ío00

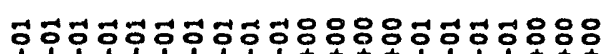

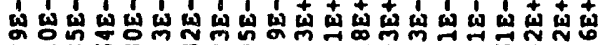

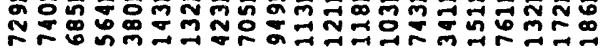
i i i i o00000000iilii

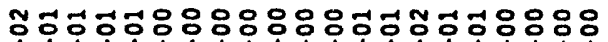

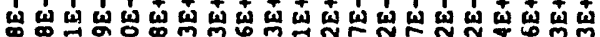

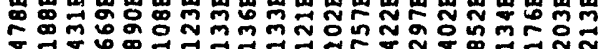
- i i i i i i i i i000000

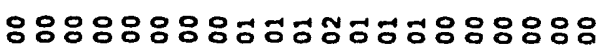

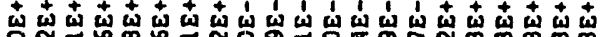
주듀유.

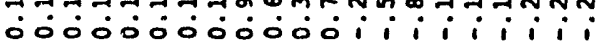

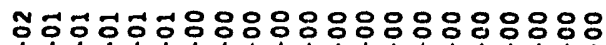

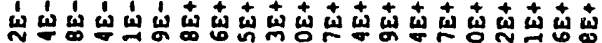

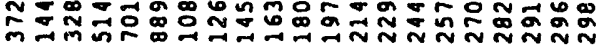
i 00000000000000000000

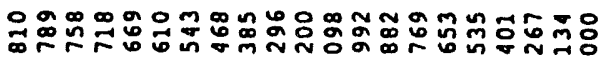

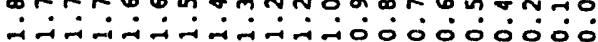

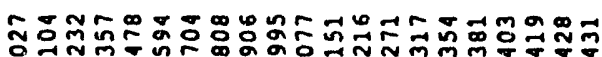

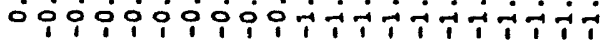

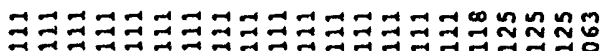

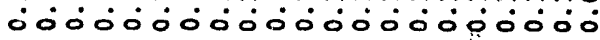

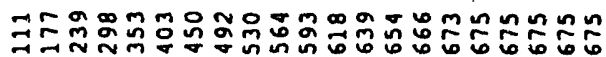

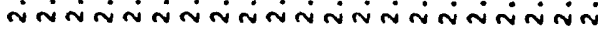

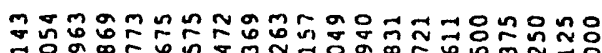

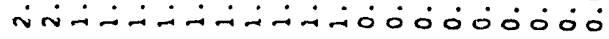

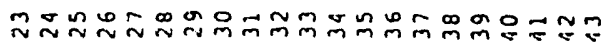




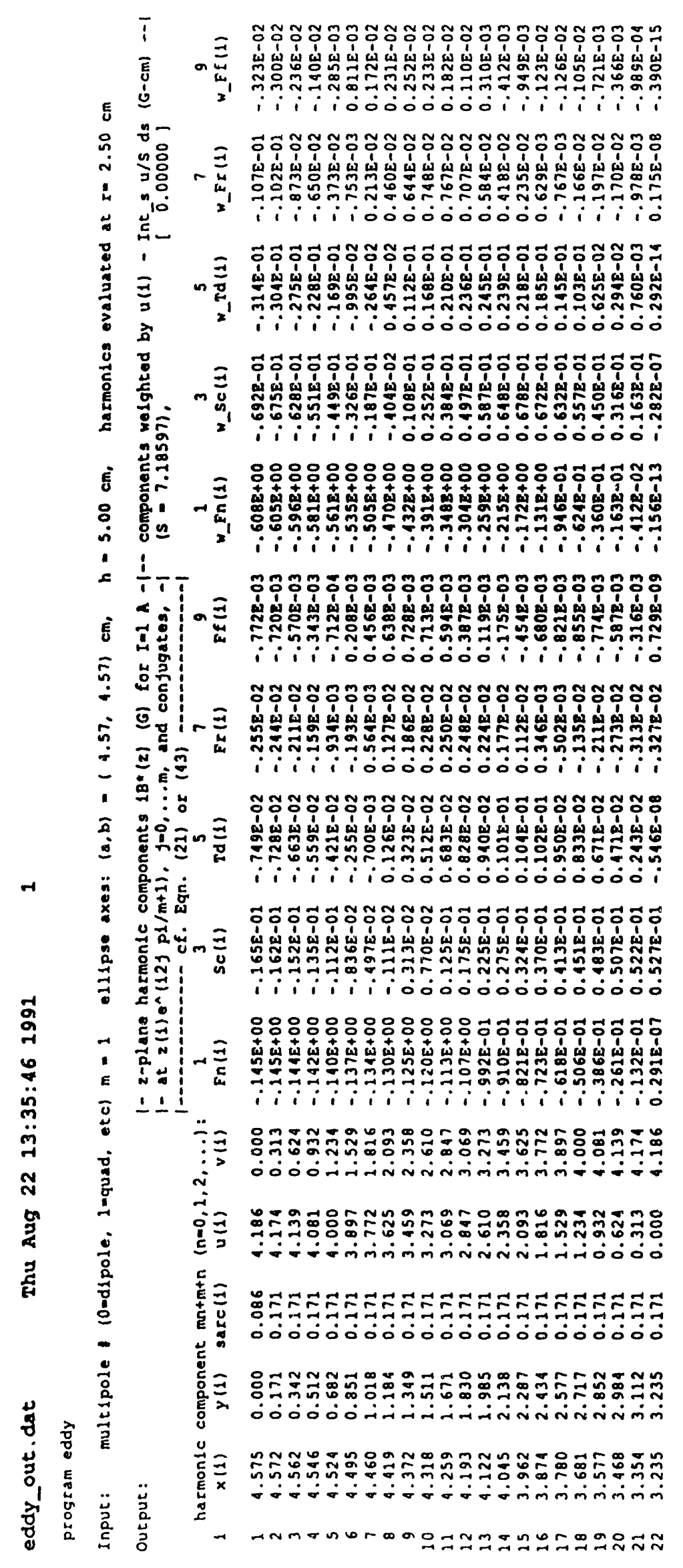

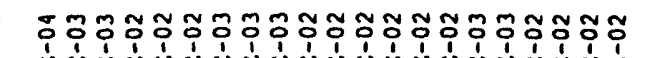

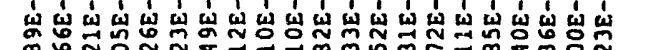
क̊ำ i i i i i $100000001 i 1$ i

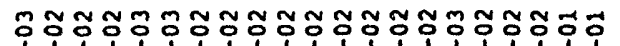

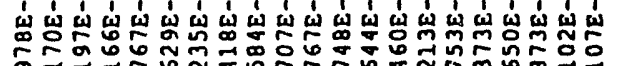

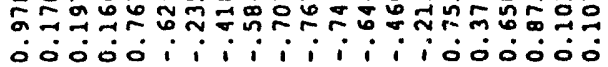

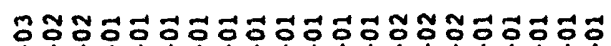

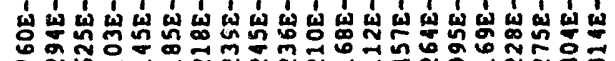

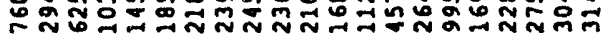
oojodoododoodoili i i i

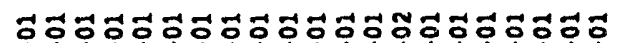

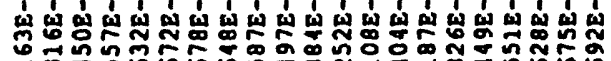

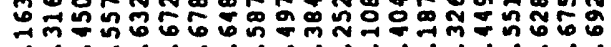
i i i i i i i i i i i iojóodó

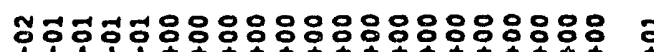

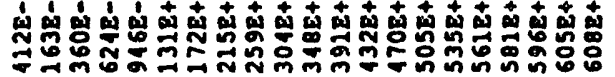

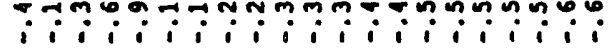

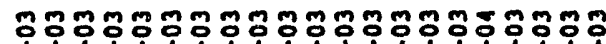
舟

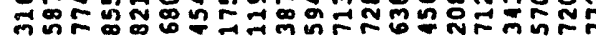
ojojojooiliilitiojojo

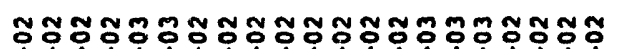

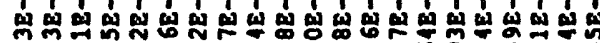

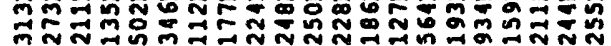
i i i ioojoojocooi i i i

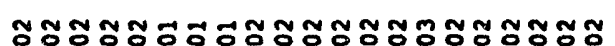
嵌

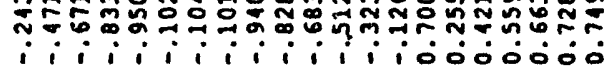

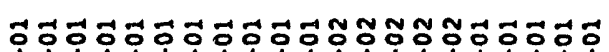

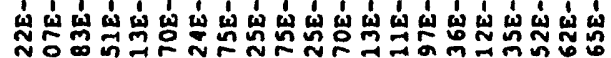

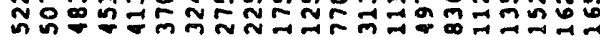
ojojoojoojoodi i i i i i

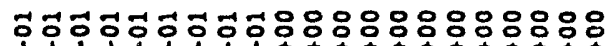

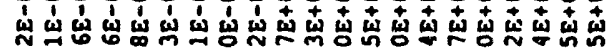

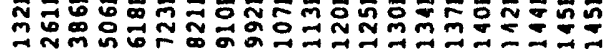
0000000000000000000

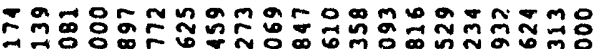

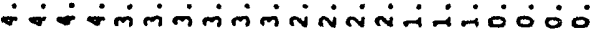

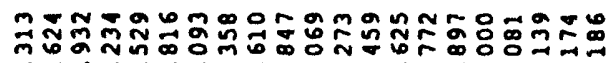

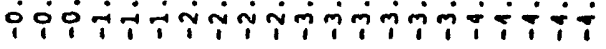

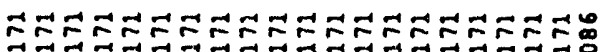

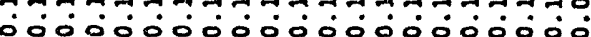

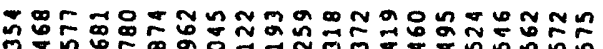

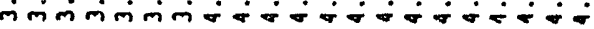

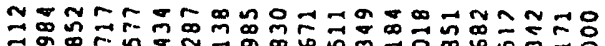

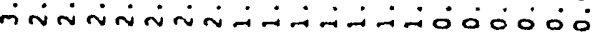

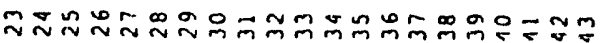




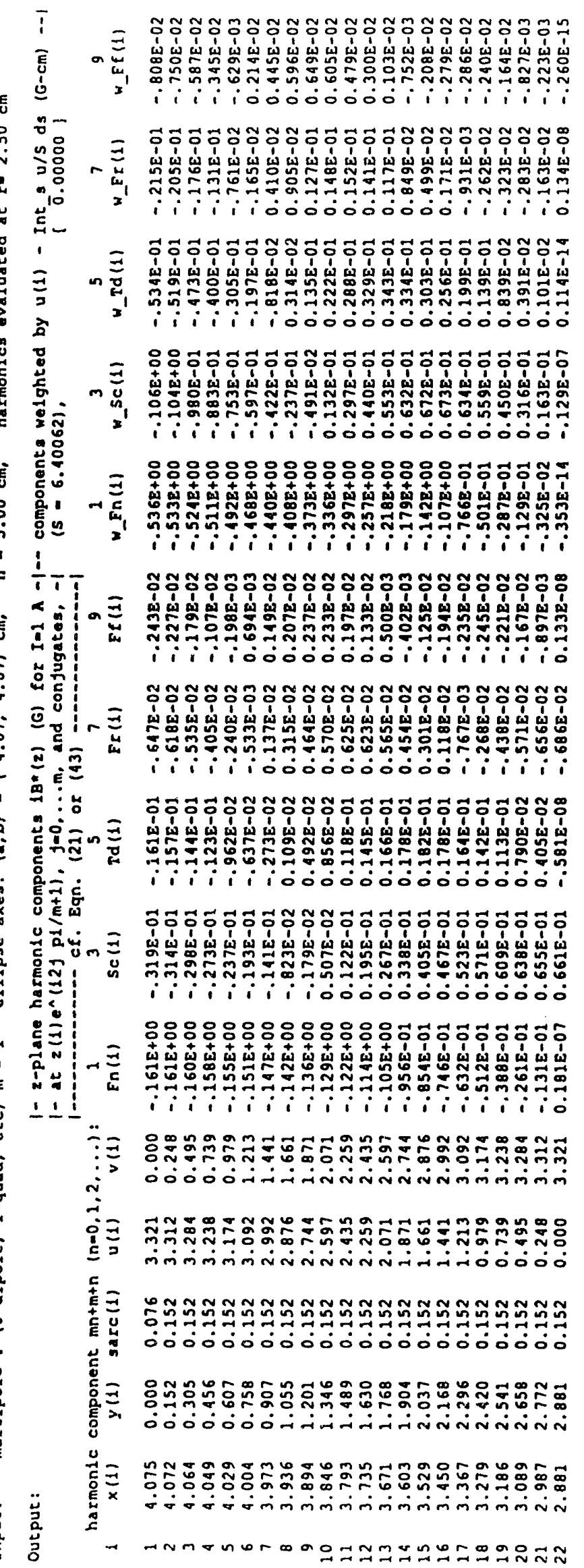

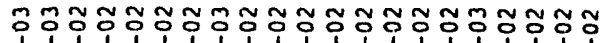

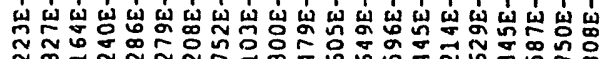
iiiiiiiiojó0000iiii

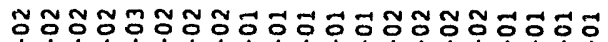

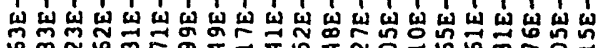

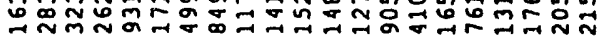
o0000ili i i i i iojojó

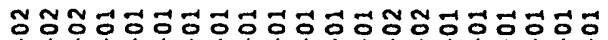

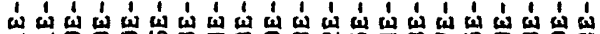
పే⿵్లై o0000000000000ili i i i

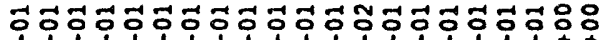

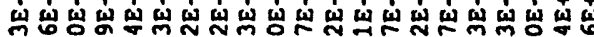

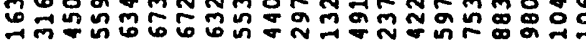
iiiiiiiiiiiiojojojojo

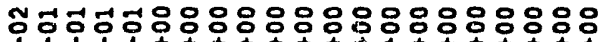
由्

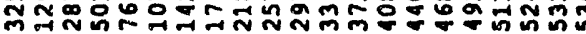
$i i_{i} i i_{i} i i_{i} i_{i} i_{i}$ i

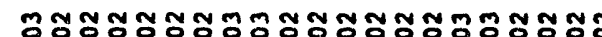
1日 क्

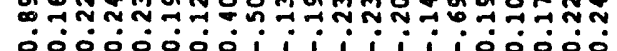

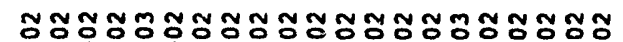
1

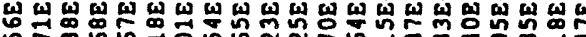

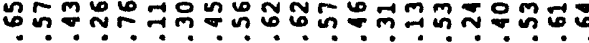
i i i i iojodocodooi i i i i

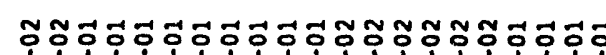

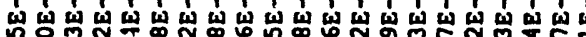

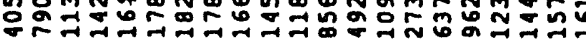

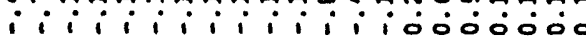

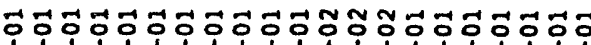

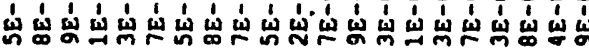

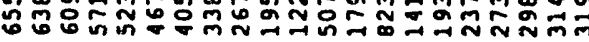
dodoojodoojoili i i i i

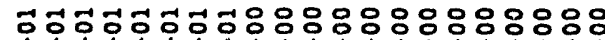

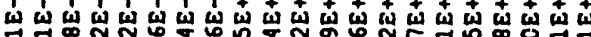

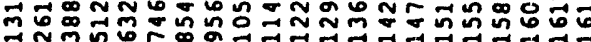
-000000000000000000

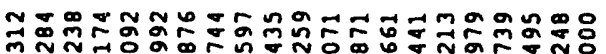

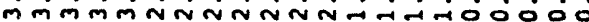

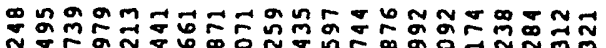

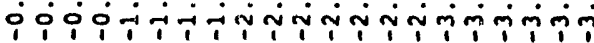

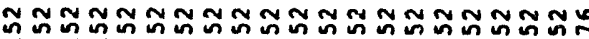

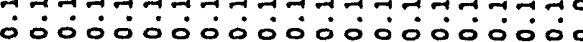

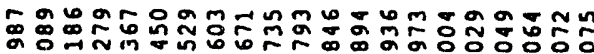

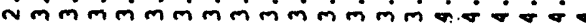

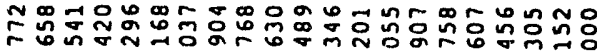

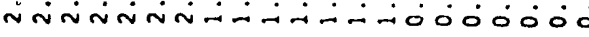

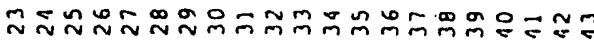




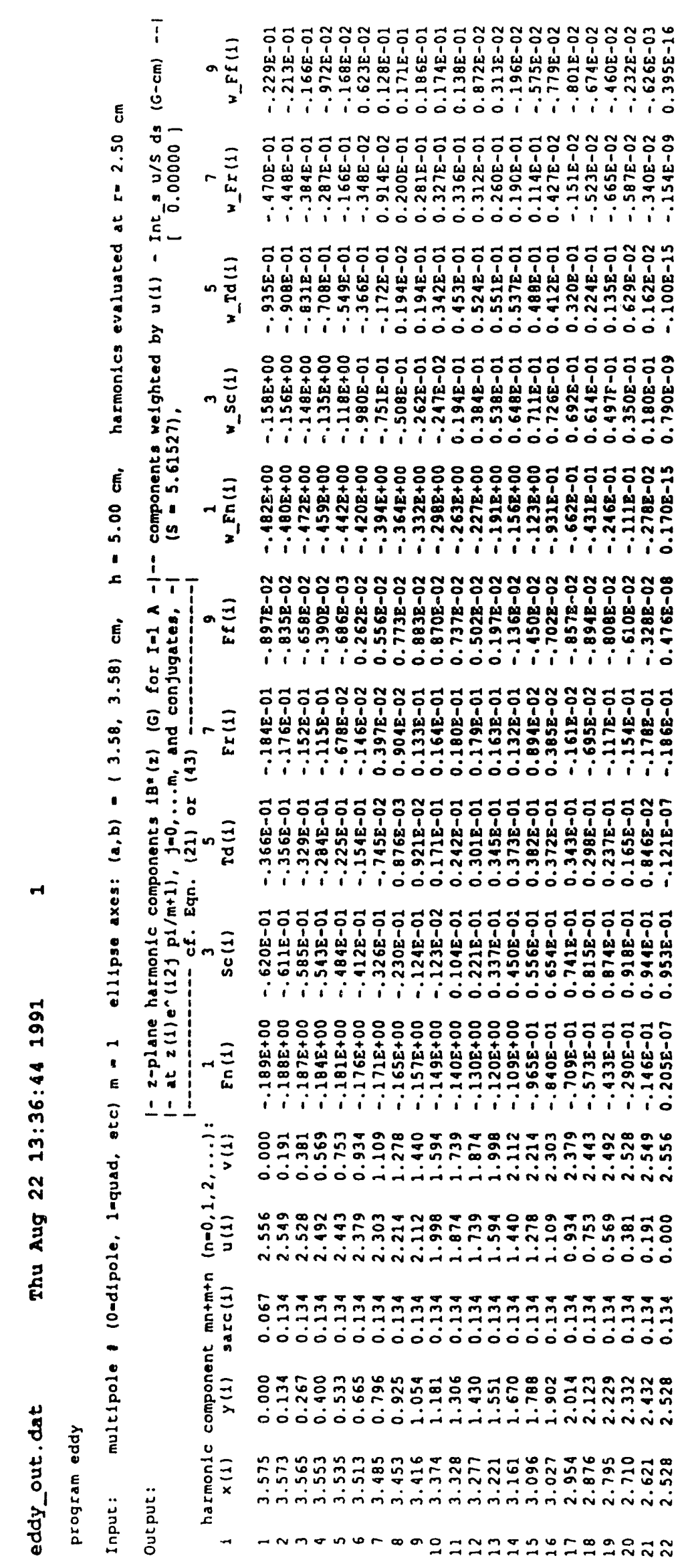

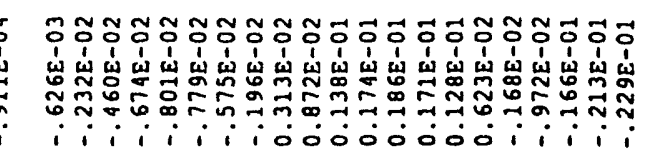

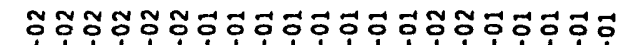

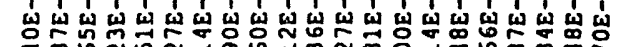

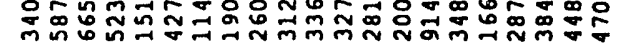

oojooiliiiiiiiiojoodo

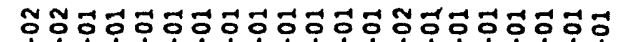

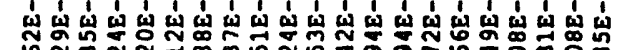

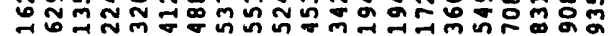

O0000000000000ili i i i

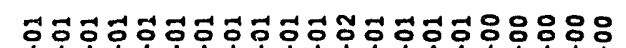

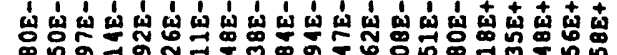

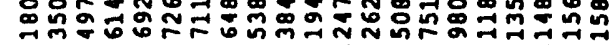

iiiiiiiliiiojojojojó

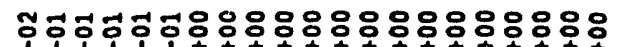

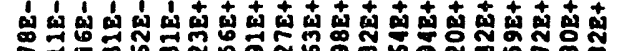

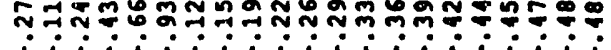

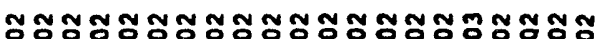

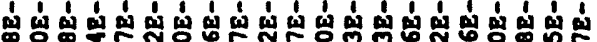

代

o0000000iii i i i ioojó

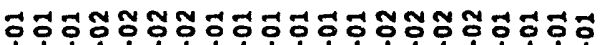

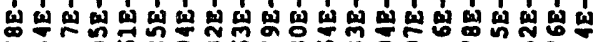

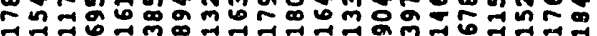

77 行

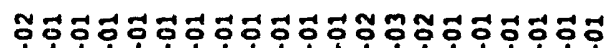

边

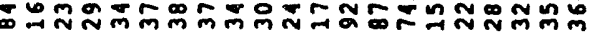

i i i i i i i i i iojoodoo

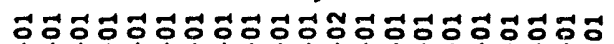

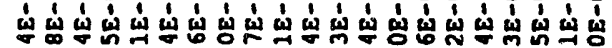

ลัส oodoojoodooi i i i i i i i i

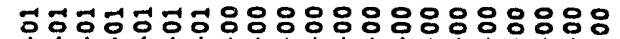

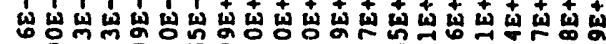

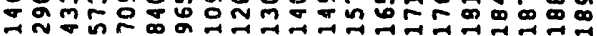

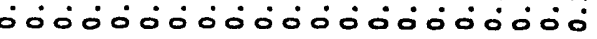

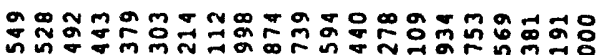

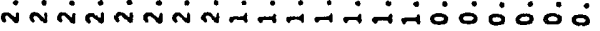

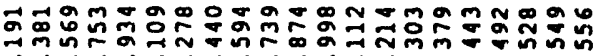

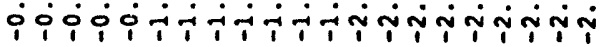

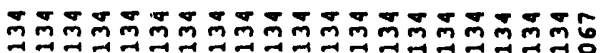

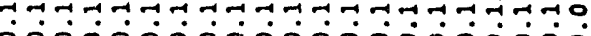

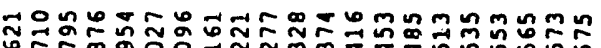

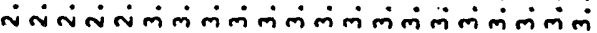

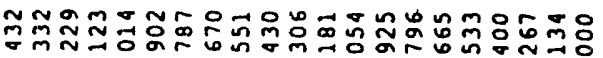

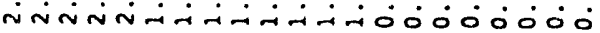

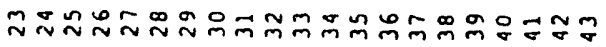




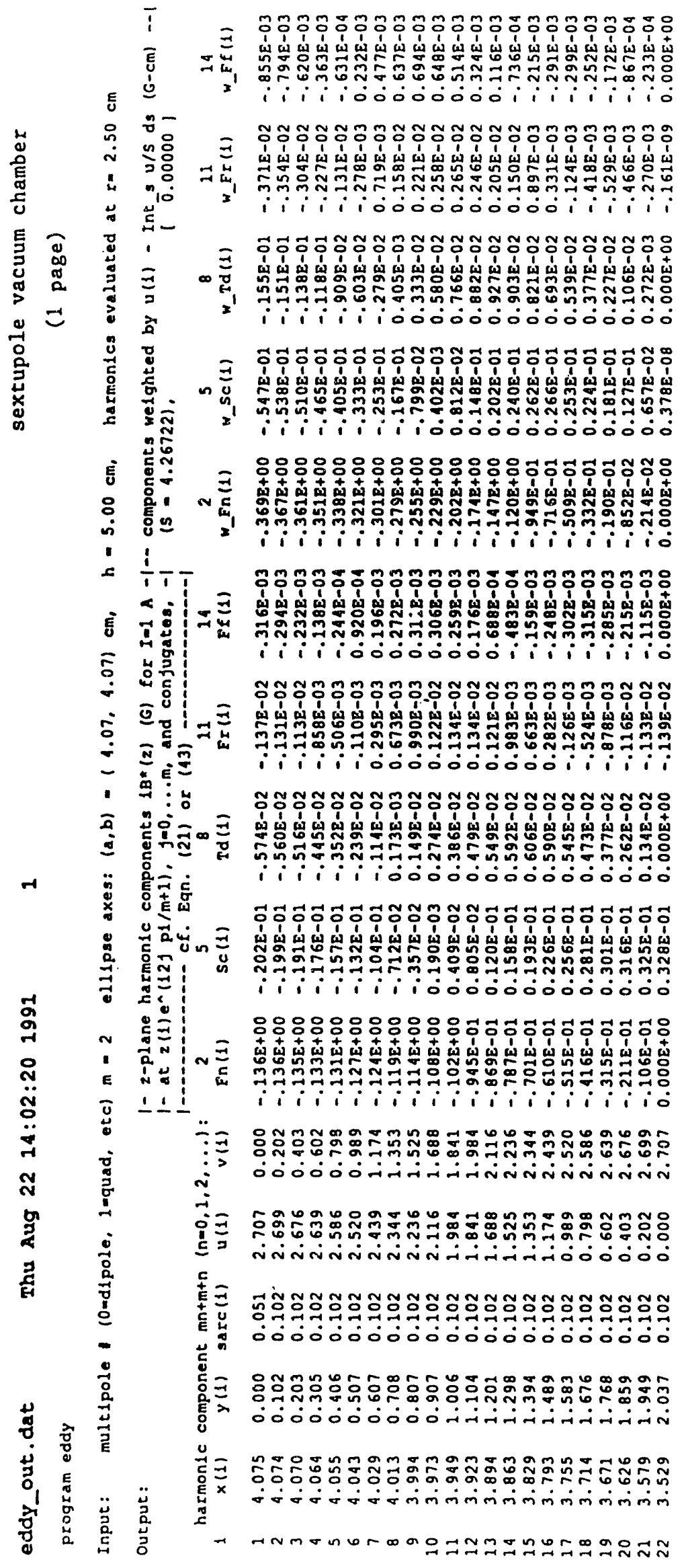

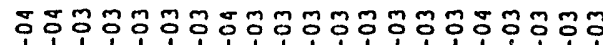

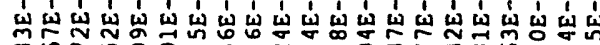

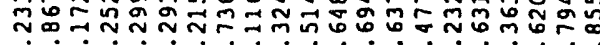
$i i i i i i$ i o000000ii i

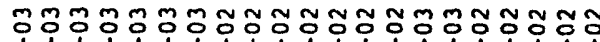

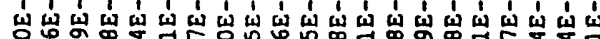

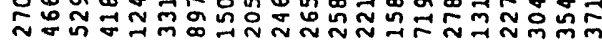
o0000i ili ili iojo:00

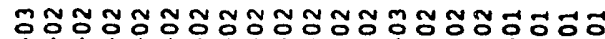

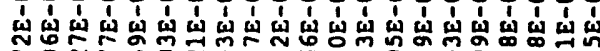

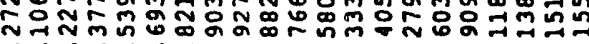
o00000000000i i i i

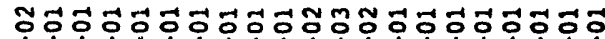

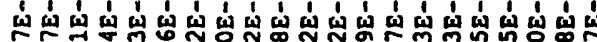

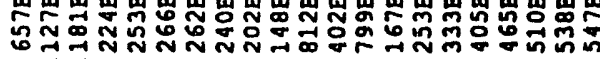
i i i i i i i ioojo0000

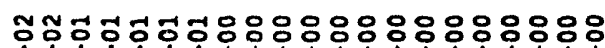

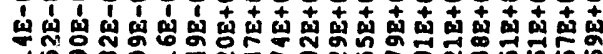

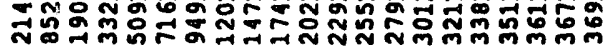
i i i i i i i i i i i i

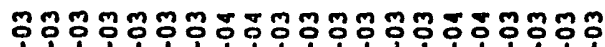

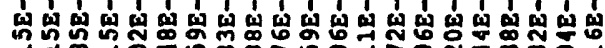

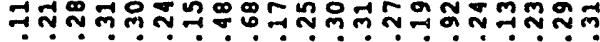
o0000000ii i i i í0000

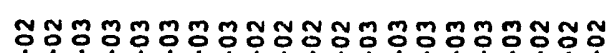

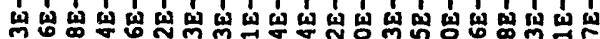

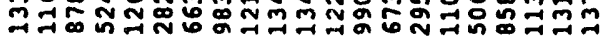

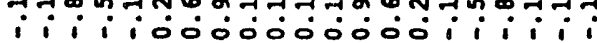

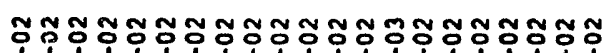

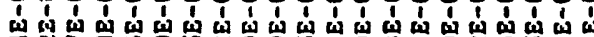

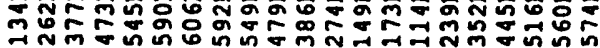
i i i i i i i i i iojó000

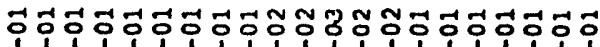

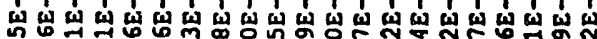

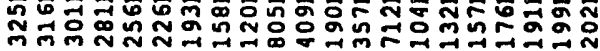

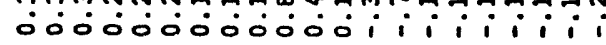

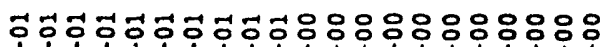

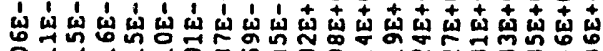

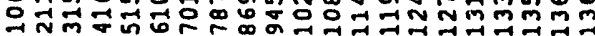

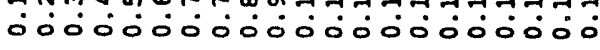

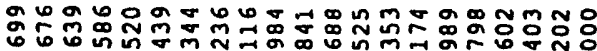

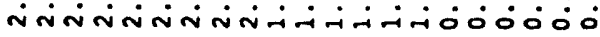

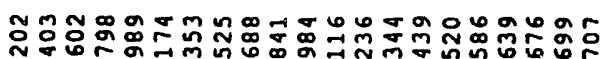
ióiö

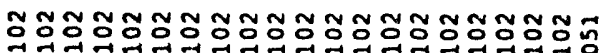
000000000000000

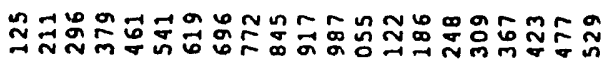

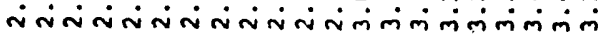

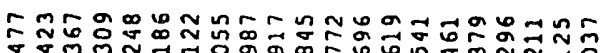

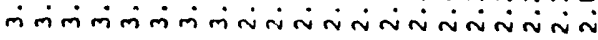

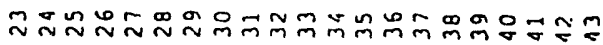




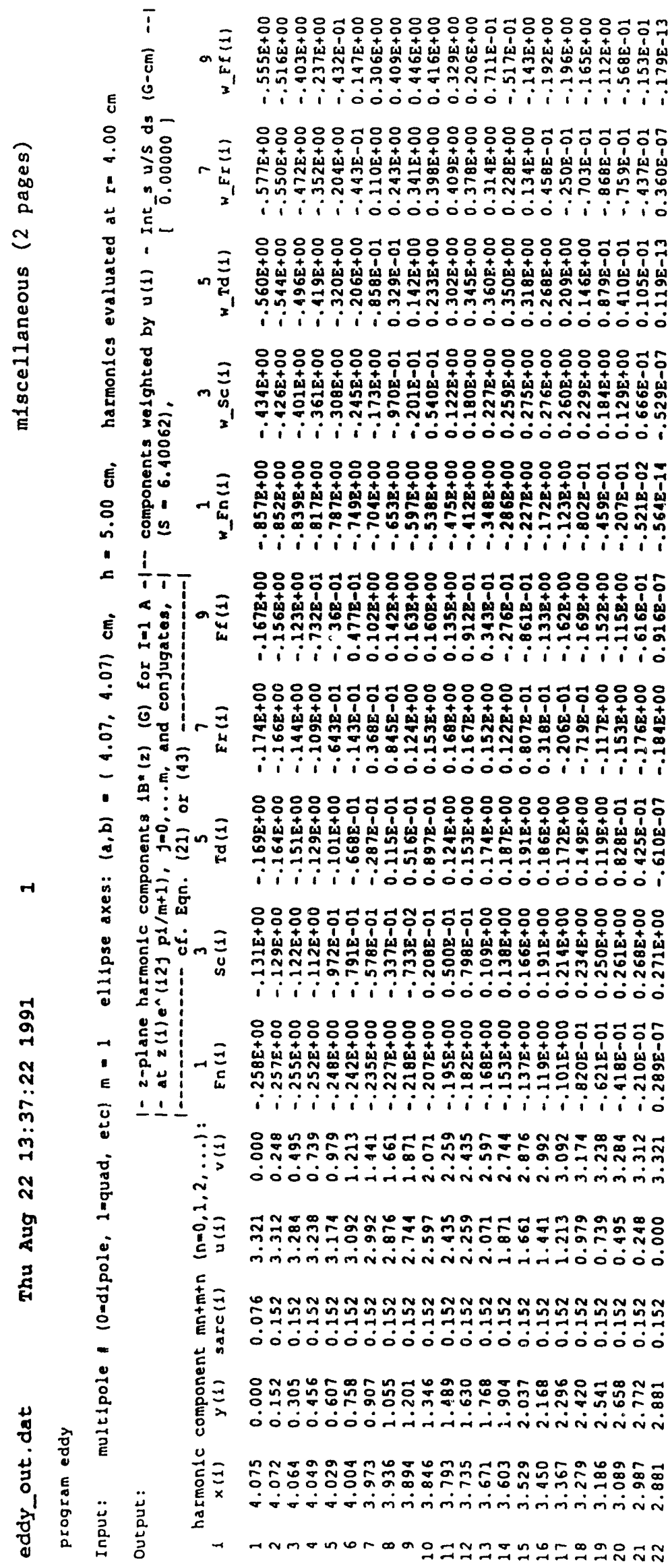

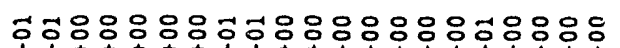

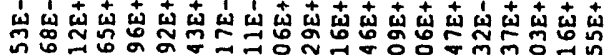

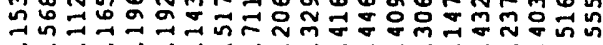

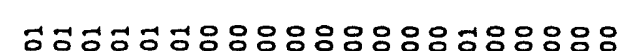

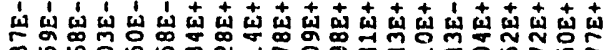

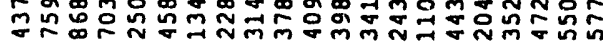

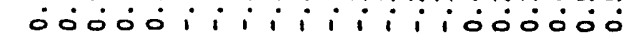

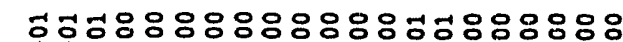

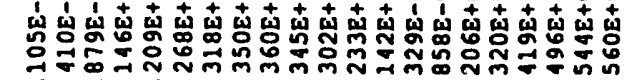

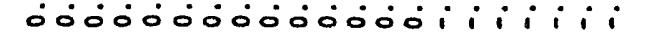

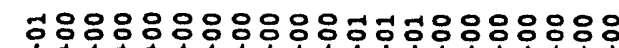

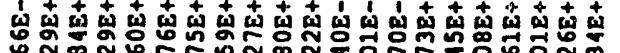

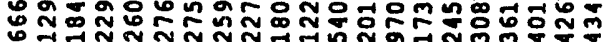

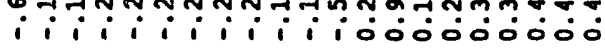

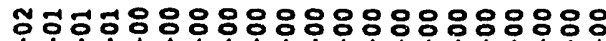

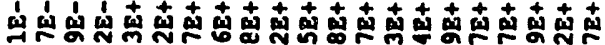

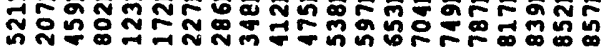
i i i i ? i i i i i i i

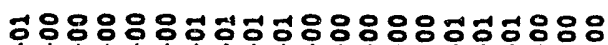

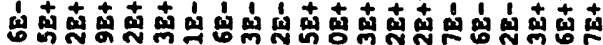

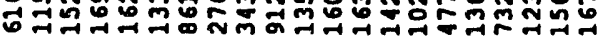

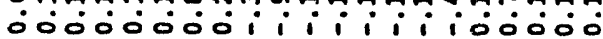

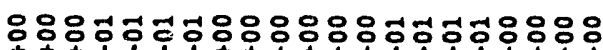

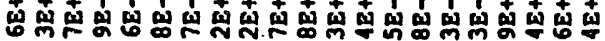

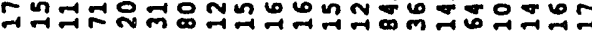
$i i i$ io00000000ii i i

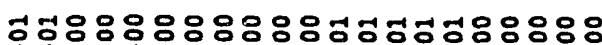

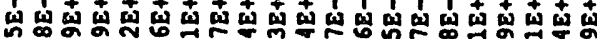

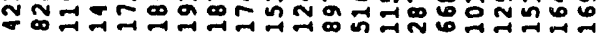
i i i i i i i i iojo0000

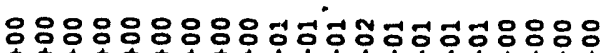

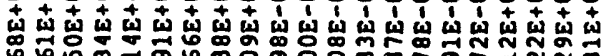

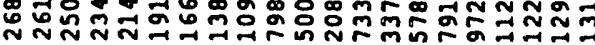
000000000000i i i i i i

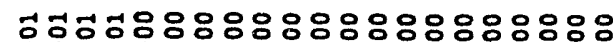

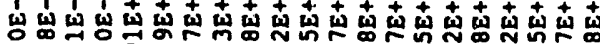

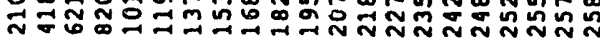
000000000000000000

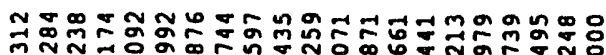

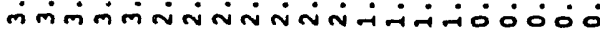

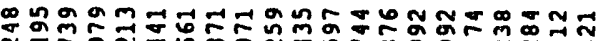

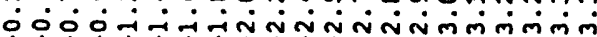
Tิ

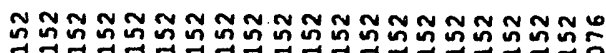

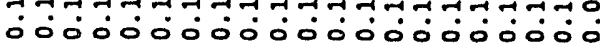

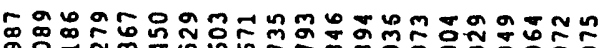

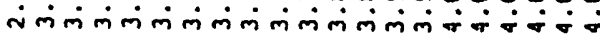

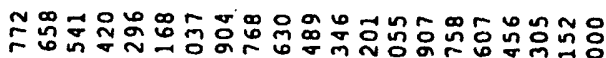

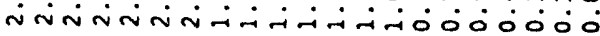

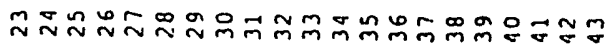

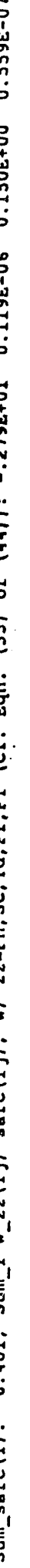




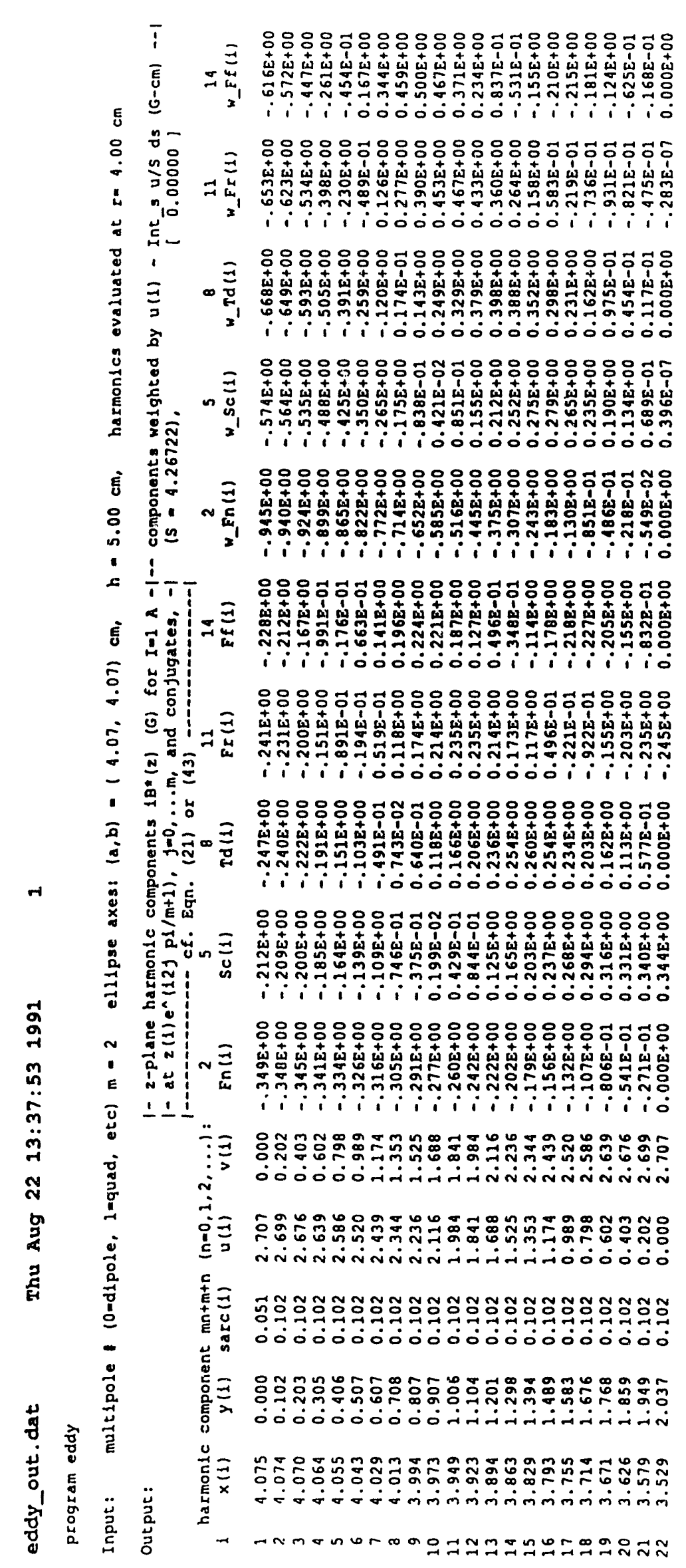

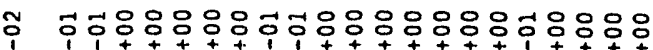

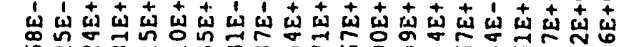

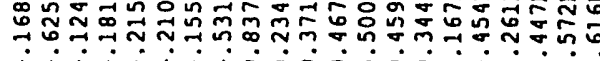
111111000000001111

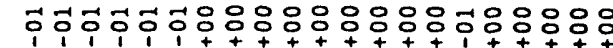

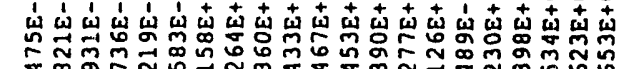
00000iiiiiiii 00000

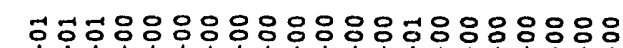

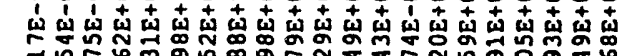

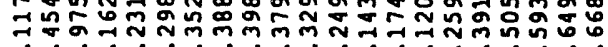

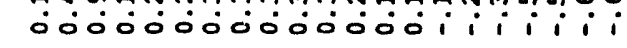

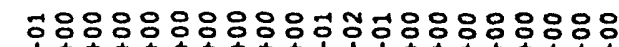

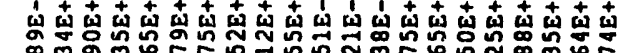

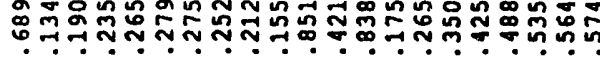

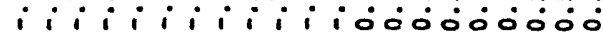

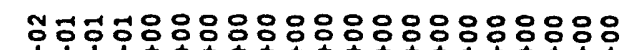

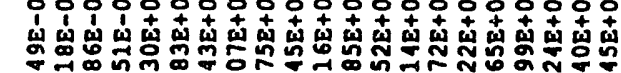

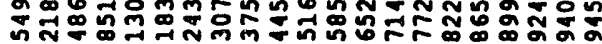

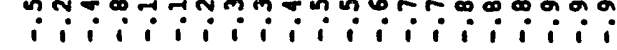

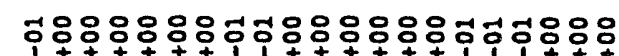

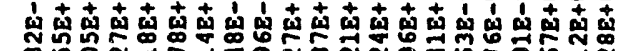
స్ల. -0000000i ili i i0000

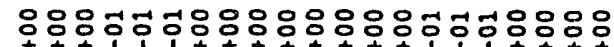

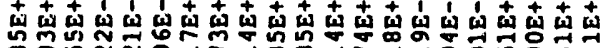

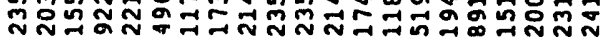

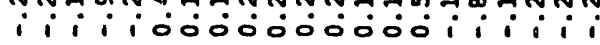

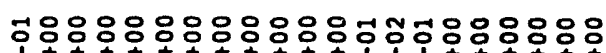

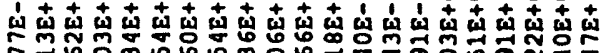

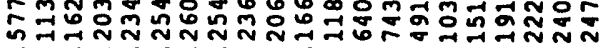
iiiiiiiiiiiio00000

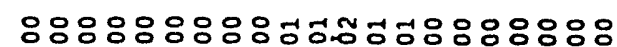

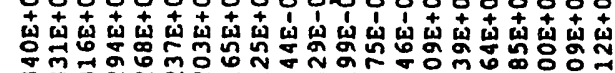

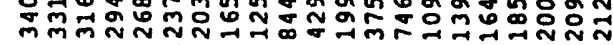

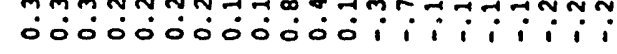

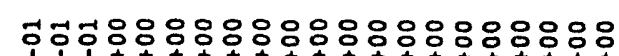

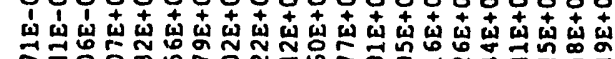

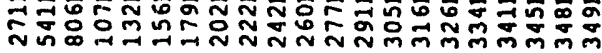

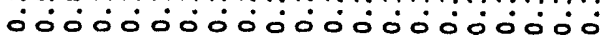

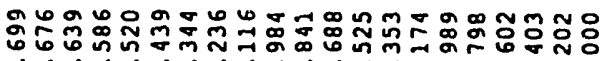

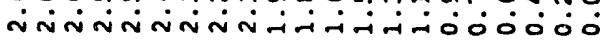

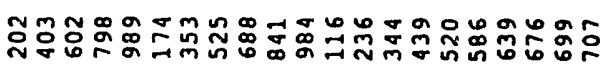

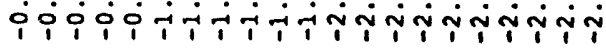

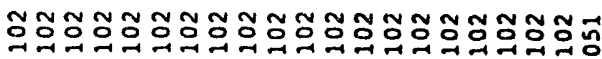
ó00000000000000

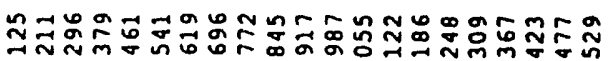

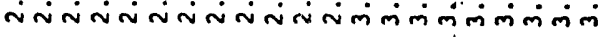

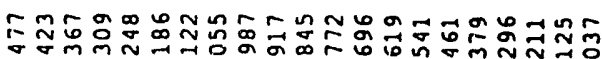

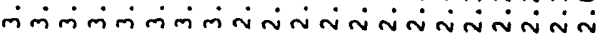

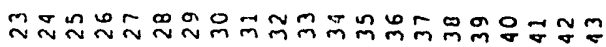




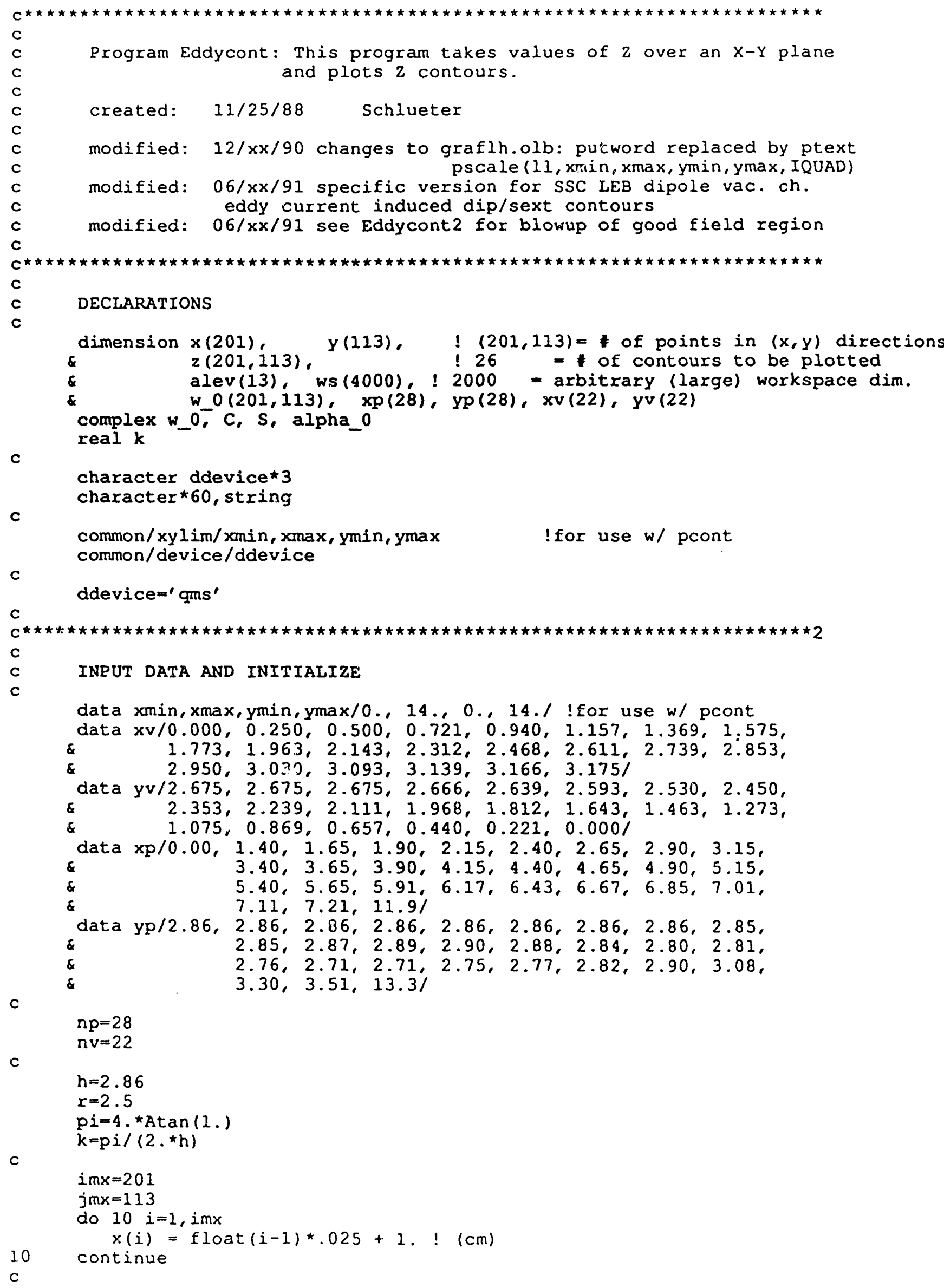




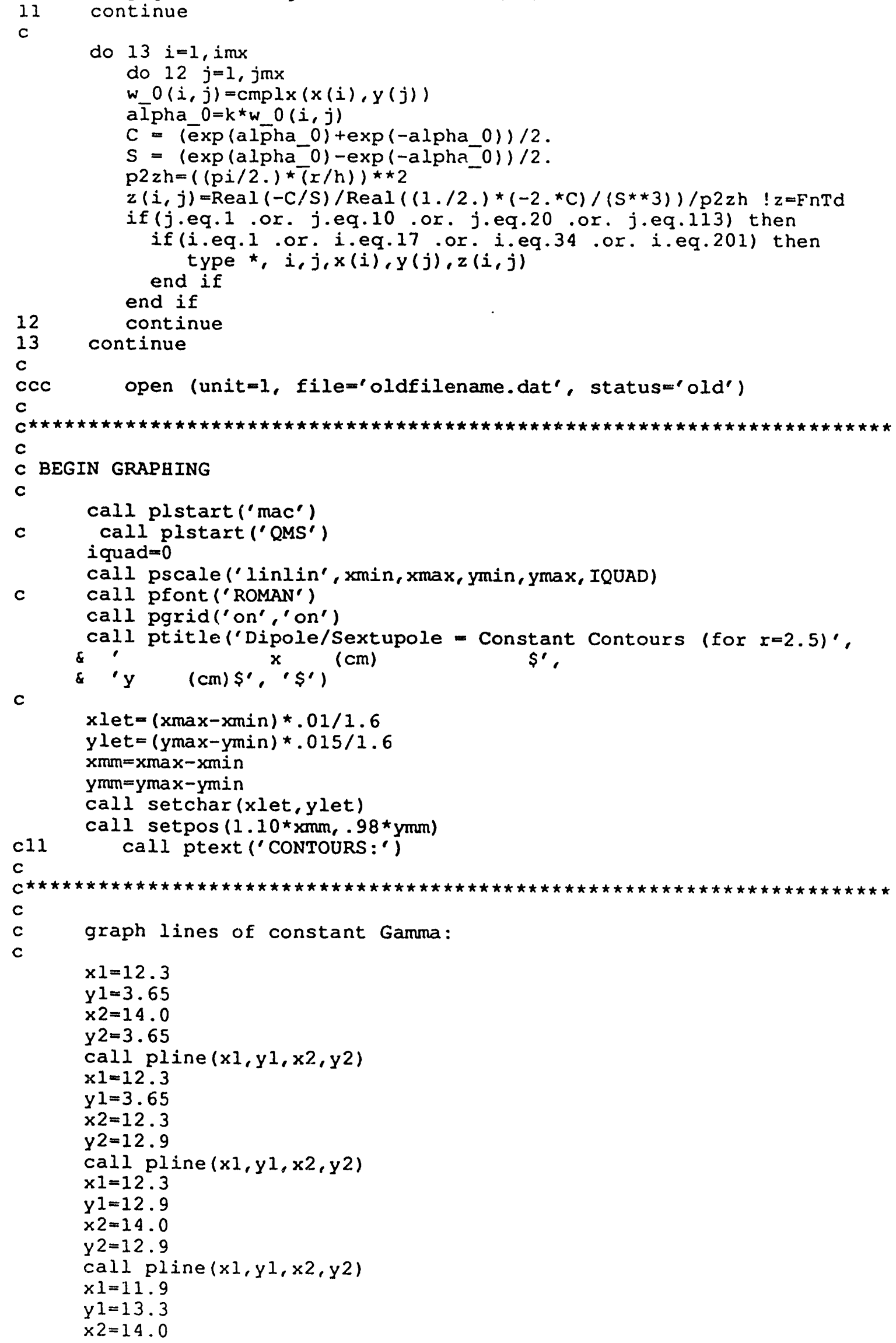


$y 2=13 \cdot 3$

call pline $(x 1, y 1, x 2, y 2)$

call pcur (xp,yp,np)

call pcur $(x v, y v, n v)$

ilev $=13$

$\operatorname{alev}(1)=-3$.

$\operatorname{alev}(2)=-5$.

$\operatorname{alev}(3)=-7$.

alev $(4)=-9$.

alev $(5)=-11$.

$\operatorname{alev}(6)=-20$.

alev $(7)=-50$.

C

alev $(8)=-1$.

$\operatorname{alev}(9)=-2$

C

alev (10) $=20$.

$\operatorname{alev}(11)=5$.

$\operatorname{alev}(12)=50$.

$\operatorname{alev}(13)=10$

C

call pstyle('solid')

call pcont('linlin', $x, y, z$, imx, imx, jmx,

C

alev, ilev, iquad, ' $\$$ ', ' $\$$ ', ' $\$$ ', ' $\$$ ')

$x$ let $=(x \max -x \min ) * .01 / 1.6$

ylet $=(y \max -y \min ) * .015 / 1.6$

call setchar ( $x$ let, ylet)

call setpos $(1.06 * x \mathrm{xm}, .95 * \mathrm{ymm})$

$\operatorname{ccc}$

call ptext ( $\left.-1 / 3,-1 / 4,-1 / 5,-1 / 6,-1 / 7,-1 / 8,-1 / 9,-1 / 10,{ }^{\circ}\right)$

call setpos $(1.00 * x \mathrm{xm}, .95 *$ ymm $)$

$\operatorname{ccc}$

call ptext ('1/inf, $\left.1 / 200,1 / 100,1 / 50,1 / 10^{\prime}\right)$

C

$x$ mult $=.95$

do 30 iter $=1$, ilev

xmult $=x m u l t-.02$

call setpos $(1.06 * x m m, x m u l t * y m m)$

c11

30

write (string,' (f5.0)')alev (iter)

end do call ptext(string)

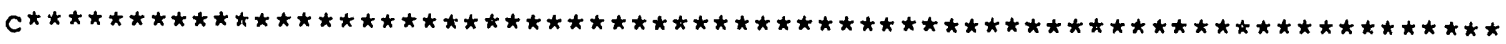

c

c

TITLES

$x$ let $=(x \max -x \min ) \star .01$

$y$ let $=(y \max -y \min ) * .015$

$x m m=x \max -x \min$

$y m m=y \max -y m i n$

call setchar ( $x$ let, $y$ let)

call setpos $(.30 * x \mathrm{~mm}, .97 * y \mathrm{~mm})$

ccc call ptext ('put legend here')

call setpos $\left(.32 x^{*} \mathrm{~mm}, .935{ }^{*} y \mathrm{~mm}\right)$

ccc call ptext ('legend continued')

$\mathrm{C}$

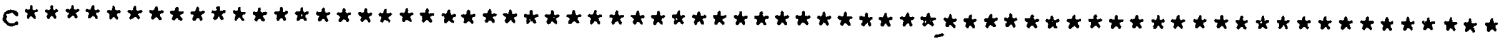

c

C

c

CONSTANTS FOR GRAPH

call setpos ( $1.06^{\star} \mathrm{xmm}, .65^{\star} y \mathrm{~mm}$ )

ccc call ptext ('input data here')

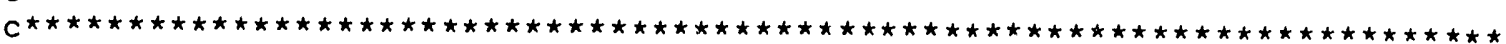

c

c

Contour Line Labels 
call setpos $(.64 * x \mathrm{~mm}, .67 * y \mathrm{~mm})$

ccc call plineh $(.64 * x \mathrm{~mm}, .68 * y \mathrm{~mm}, .60 * x \mathrm{~mm}, .64 * y \mathrm{~mm})$

$\mathrm{CCC}$ call ptext ('z contour')

C

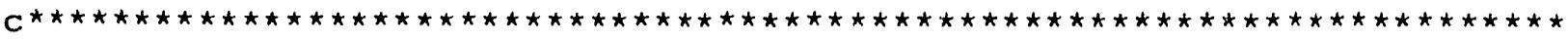

$C$

call pclose

c

close (unit $=1$, status=' keep') $^{\prime}$

C

end

$c^{*}$ 


\section{Appendix D: Elliptical Vacuum Chamber Eddy Current Induced Harmonics and their Suppression for a Sextupole Electromagnet}


a) z-plane (sextupole)

b) w-plane (dipole)
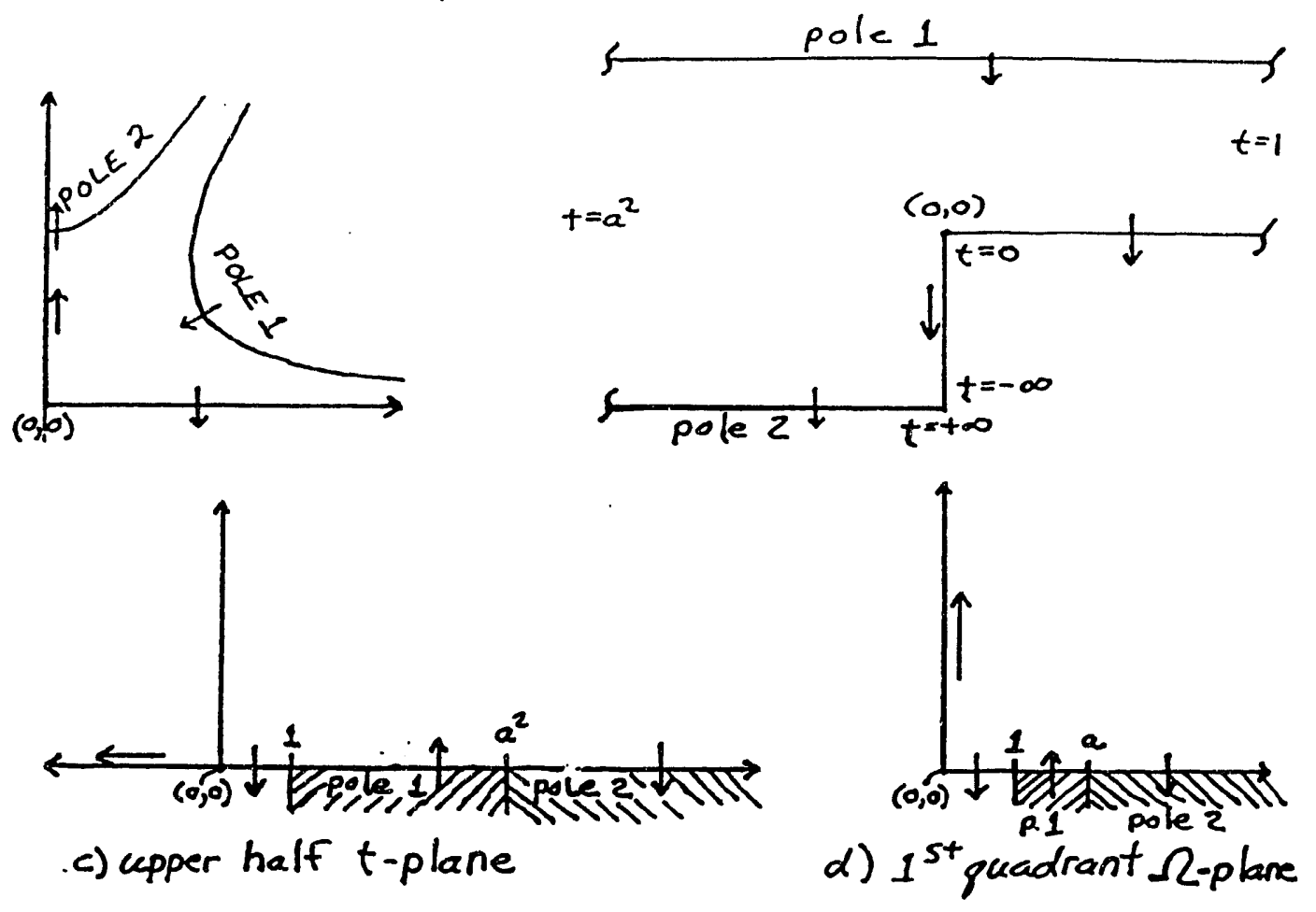

Figure 17. Sextupole magnet with elliptical vacuum chamber: (a) lab frame $z$-plane, (b) dipole frame $w$-plane, (c) upper half $t$-plane, (d) first quadrant $\Omega$-plane

\section{Appendix D: Elliptical Vacuum Chamber Eddy Current Induced Harmonics and their Suppression for a Sex- tupole Electromagnet}

For the sextupole magnet with an elliptical vacuum chamber in the laboratory $z$-plane (Figure 17a), we use the transformation ${ }^{5} w=z^{3} / r_{p}^{2}$, where $r_{p}$ is the-sextupole aperture radius, to map to a dipole $w$-plane geometry (Figure 17b). The Schwarz-Christoffel transformation

$$
\frac{d w}{d t}=\frac{(1 / k) \sqrt{t}}{\left(t-a^{2}\right)(t-1)}
$$

maps the region of interest of the dipole $w$-plane to the upper half $t$-plane (Figure $17 \mathrm{c}$ ). Vacuum chamber eddy current induced magnetic field lines are perpendicular to the $t$ plane's positive abscissa, but parallel to its negative abscissa. A mapping function $\Omega=\sqrt{t}$ results in field lines in the iron-free $\Omega$-plane perpendicular to the positive abscissa, parallel to the positive ordinate, and thus perpendicular to the negative abscissa as well (Figure 17d). Contributions to th: magnetic field of current filaments are readily calculated in

\footnotetext{
${ }^{5}$ Computationally, one must be careful in transforming from $w$ back to $z$, since values in the w-plane with negative imaginary part are handled internally by the computer as having an argument $\theta:-p i<\theta<0$ (refer to Figure $17 b$ ).
while tansformationally pi $<\theta<3 \pi / 2$. Caution!
} 
the iron-free $\Omega$-plane and transformation back to the laboratory $z$-plane yields the desired solution for eddy current induced $B^{*}(z)$.

The relationship between $w$ and $\Omega$ is

$$
\frac{d w}{d \Omega}=\frac{d w}{d t} \frac{d t}{d \Omega}=\frac{(2 / k) t}{\left(t-a^{2}\right)(t-1)}=\frac{1}{k\left(1-a^{2}\right)}\left[\frac{-a}{\Omega-a}+\frac{a}{\Omega+a}+\frac{1}{\Omega-1}+\frac{-1}{\Omega+1}\right]
$$

Now $\operatorname{Im}\left(\left.\Delta w\right|_{t=a^{2}}\right)=-2 r_{p}=\frac{ \pm \pi a}{k\left(a^{2}-1\right)}$ and $\operatorname{Im}\left(\left.\Delta w\right|_{t=1}\right)=r_{p}=\frac{ \pm \pi}{k\left(1-a^{2}\right)}$ so we have $a= \pm 2, \frac{1}{k}=\frac{ \pm 3 r_{p}}{\pi}$ and

$$
\frac{d w}{d \Omega}=\frac{-r_{p}}{\pi}\left[\frac{-2}{\Omega-2}+\frac{2}{\Omega+2}+\frac{1}{\Omega-1}+\frac{-1}{\Omega+1}\right]
$$

Integrating, we have

$$
\frac{\pi}{r_{p}} w=2 \ln (\Omega-2)-2 \ln (\Omega+2)-\ln (\Omega-1)+\ln (\Omega+1)-\pi i
$$

This equation's format is useful for numerical work since on the computer $-\pi<$ $\operatorname{Im}(\ln (f(\Omega)) \leq \pi$ and $\Omega$ values are restricted to the first quadrant; combining the terms under just one or two 'In' signs leads to transformation errors by factors of $\pi i$ : Caution! We have $w=f_{1}(\Omega)$; obtaining $\Omega=f_{2}(w)$ comprises solving the complex cubic equation in $\Omega$ for the appropriate root. Figure 18 shows the overall $z-\Omega$ mapping.

For current filaments in the $\Omega$-plane of magnitudes $+I$ at $\Omega_{1}$ and $-I$ at $-\Omega_{1}^{*}$ and their images about the midplane:

$$
F(\Omega)=\frac{-I}{2 \pi} \ln \left[\frac{\left(\Omega-\Omega_{1}\right)\left(\Omega-\Omega_{1}^{*}\right)}{\left(\Omega+\Omega_{1}^{*}\right)\left(\Omega+\Omega_{1}\right)}\right]
$$

and

$$
i F^{\prime}(\Omega)=\frac{I}{2 \pi i}\left[\frac{1}{\Omega-\Omega_{1}}+\frac{1}{\Omega-\Omega_{1}^{*}}-\frac{1}{\Omega+\Omega_{1}^{*}}-\frac{1}{\Omega+\Omega_{1}}\right]
$$

This current filament distribution in $\Omega$ is equivalent to filaments in the $z$-plane of magnitudes $+I$ at $z_{1}\left(w_{1}\left(t_{1}\left(\Omega_{1}\right)\right)\right)$ and $-I$ at $-z_{1}^{*}$ and their images about the midplane in $z$. So for this current distribution, the magnetic field in the laboratory $z$-plane is:

$$
\begin{aligned}
B^{*}(z)= & B^{*}(w) /(d z / d w)=\mu_{0} i F^{\prime}(\Omega) /(d w / d \Omega) /(d z / d w)= \\
& \frac{-3 \mu_{0} I z^{2}}{2 r_{p}^{3} i} \frac{\left[\frac{1}{\Omega-\Omega_{1}}+\frac{1}{\Omega-\Omega_{i}}-\frac{1}{\Omega+\Omega_{i}}-\frac{1}{\Omega+\Omega_{1}}\right]}{\left[\frac{-2}{\Omega-2}+\frac{2}{\Omega+2}+\frac{1}{\Omega-1}+\frac{-1}{\Omega+1}\right]}
\end{aligned}
$$



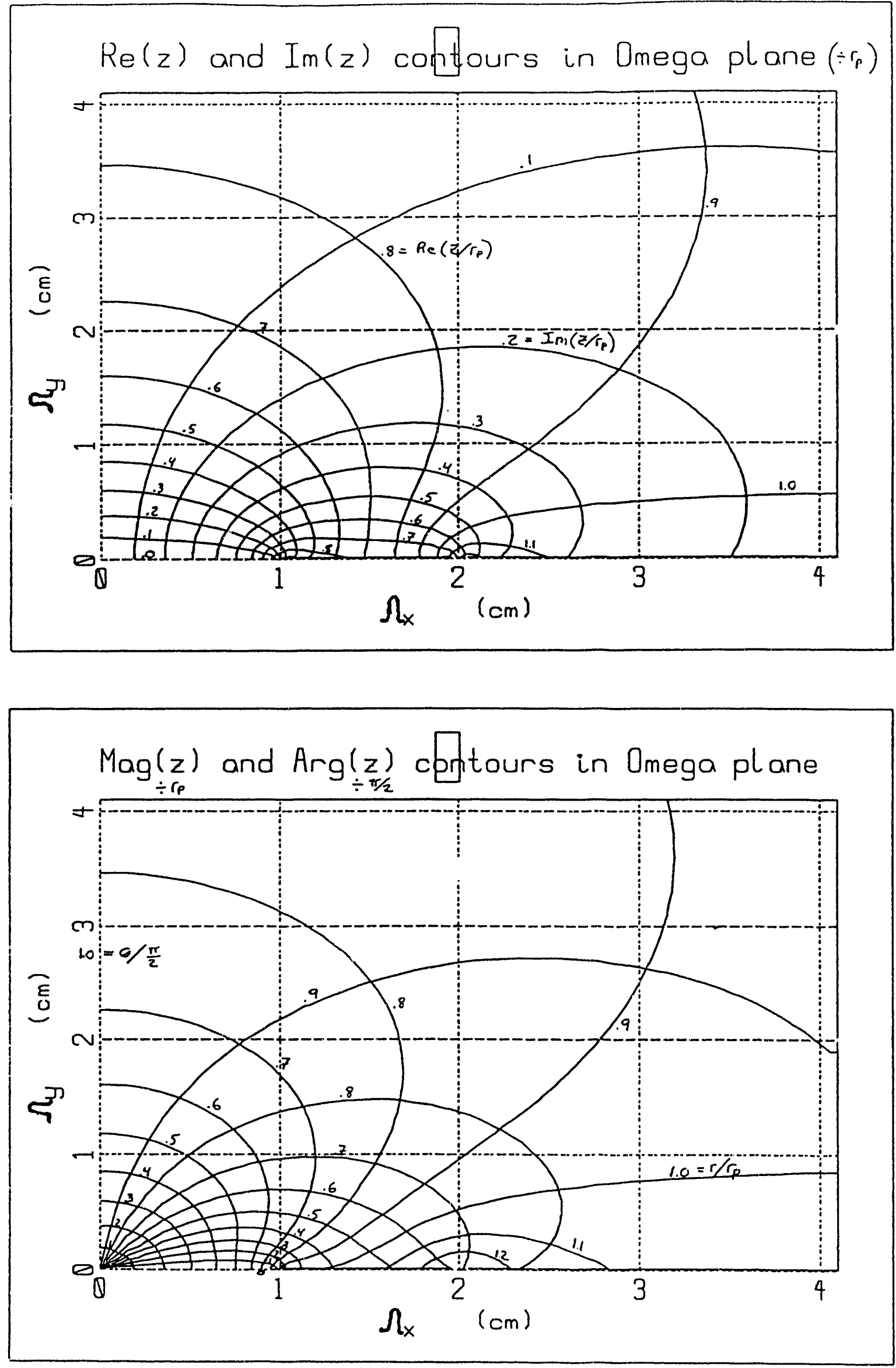

Figure 18. First quadrant of elliptical sextupole in $z$ plane conformally transformed to iron-free first quadrant $\Omega$-plane 
Using the value of $I_{\text {vac }}^{\prime}\left(s_{z}\right)$ from Eqn. (45). Eqn. (60) can be integrated over the portion of the elliptical (or any shape symmetrical with respect to both the $x$ and $y$ axes) vacuum chamber arc lying in the first quadrant of the laboratory $z$-plane to yield the eddy current induced field in a sextupole magnet.

Field values from Eqn. (60) agree beautifully with a test POISSON run on a sextupole magnet.

As $z \longrightarrow 0$, we have $w \longrightarrow 0, \frac{d w}{d t} \longrightarrow \sqrt{t} / k a^{2}, w=\frac{r_{p}}{2 \pi} t^{3 / 2}, \frac{d w}{d \Omega}=\frac{d w}{d t} \frac{d t}{d \Omega}=\frac{3 r_{p} w_{2}}{2 \pi}=$ $\frac{3 z^{2}}{(2 \pi)^{1 / 3} r_{p}}, \Omega \longrightarrow 0, i F^{\prime}(\Omega=0)=\frac{-I 2 R_{e}\left(\Omega_{1}\right)}{\pi i\left|\Omega_{1}\right|^{2}}$, and thus

$$
i B^{*}(z=0)=\left(\frac{4}{\pi}\right)^{2 / 3} \frac{\mu_{0} I}{r_{p}} \frac{R e\left(\Omega_{1}\right)}{\left|\Omega_{1}\right|^{2}}
$$




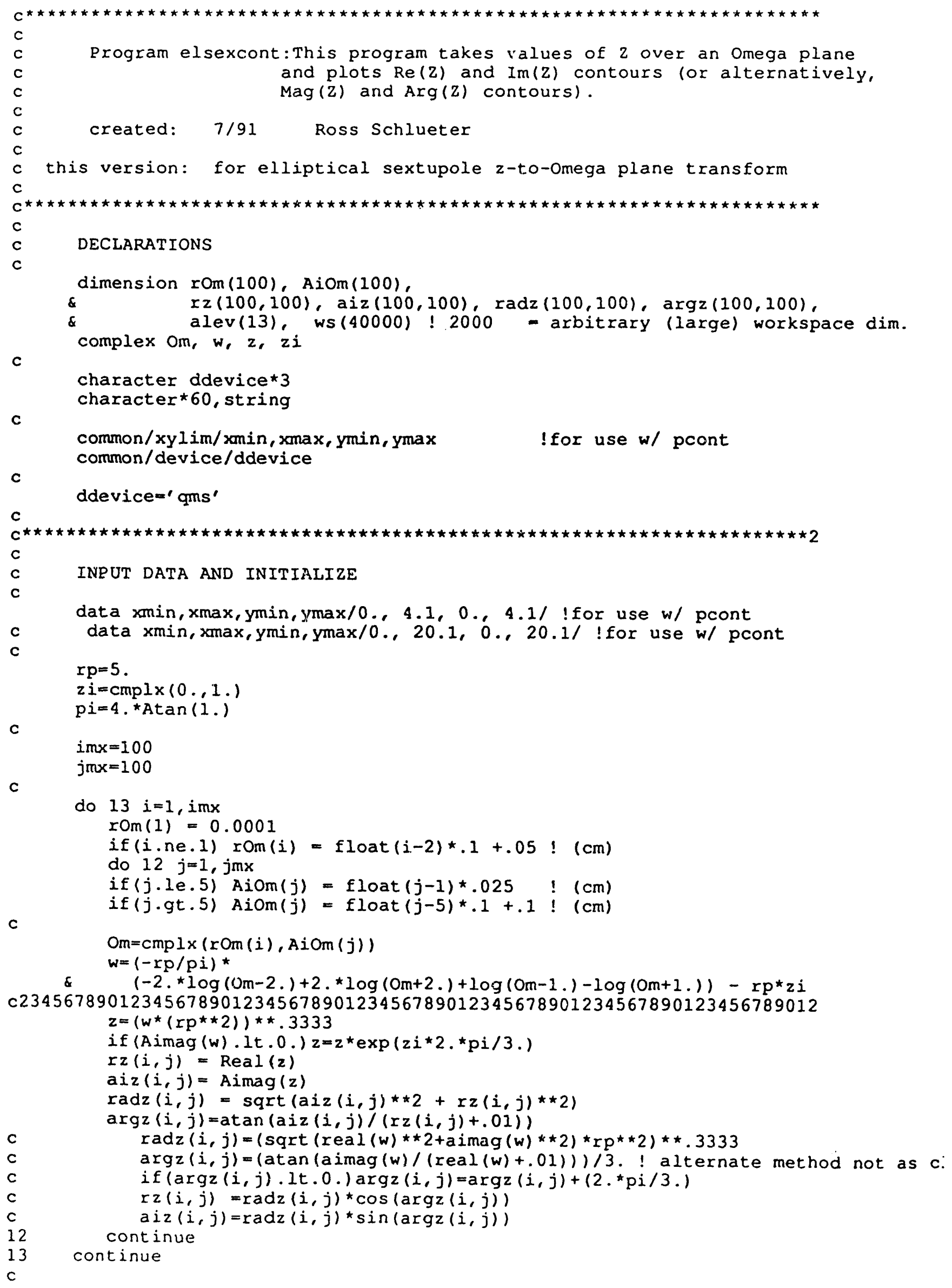




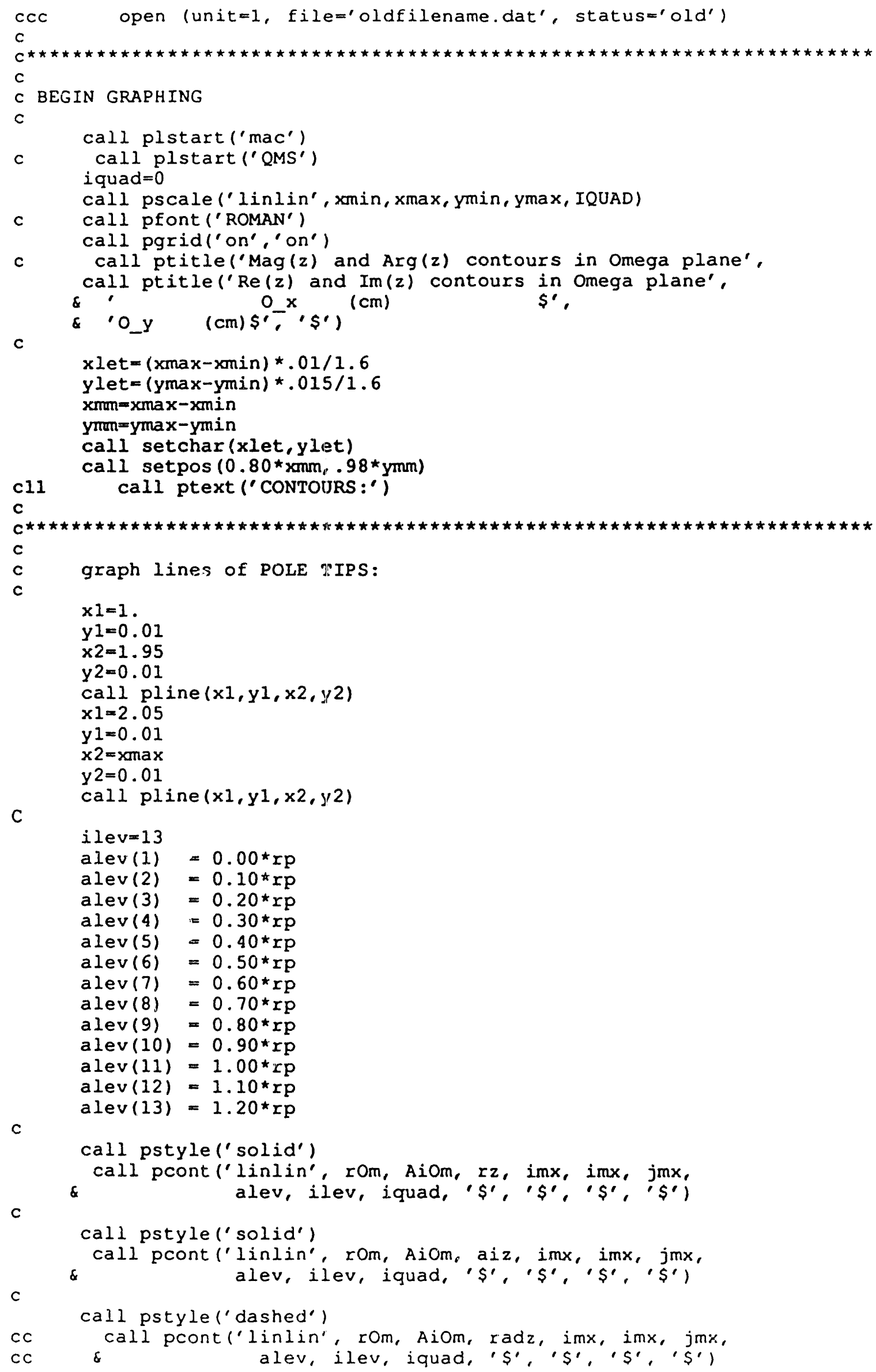


C

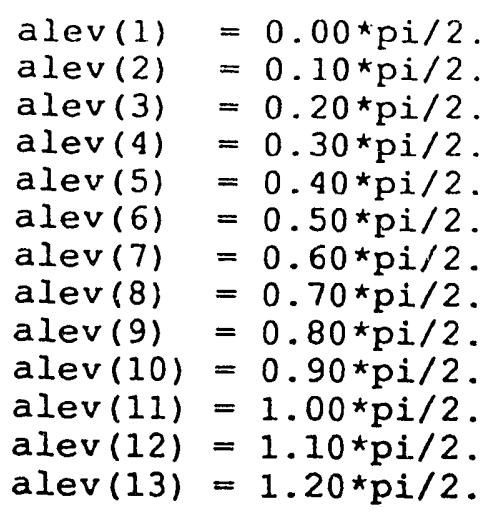

C

cC

C

c

C

call pstyle ('dashed')

call pcont('Iinlin', rOm, Aiom, argz, imx, imx, jmx, \& alev, ilev, iquad, ' $\$$ ', ' $\$$ ', ' $\$$ ', ' $\$$ ')

call pclose

close (unit $=1$, status $=$ ' $^{\text {keep') }}$

end

$c^{*}$ 


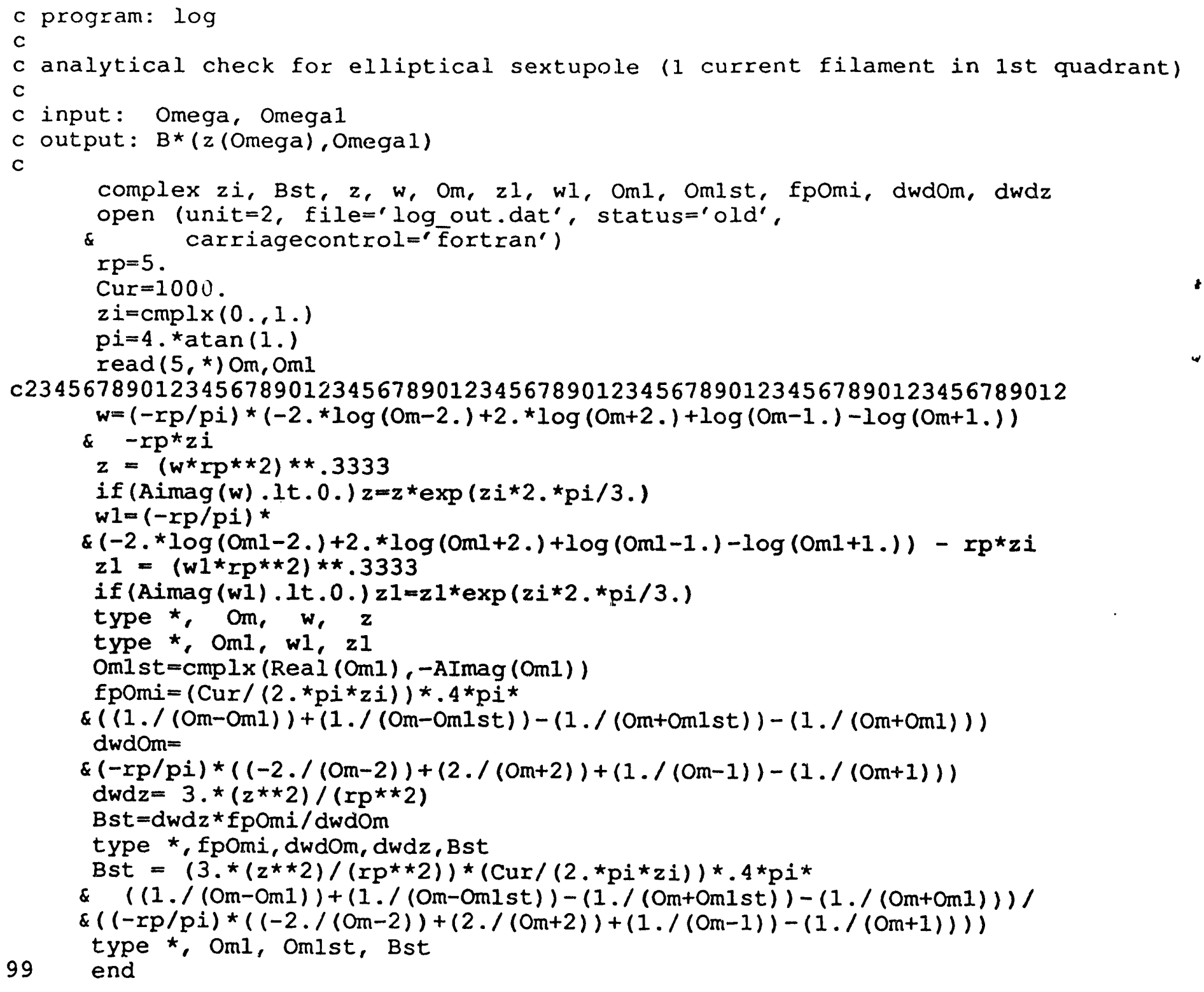



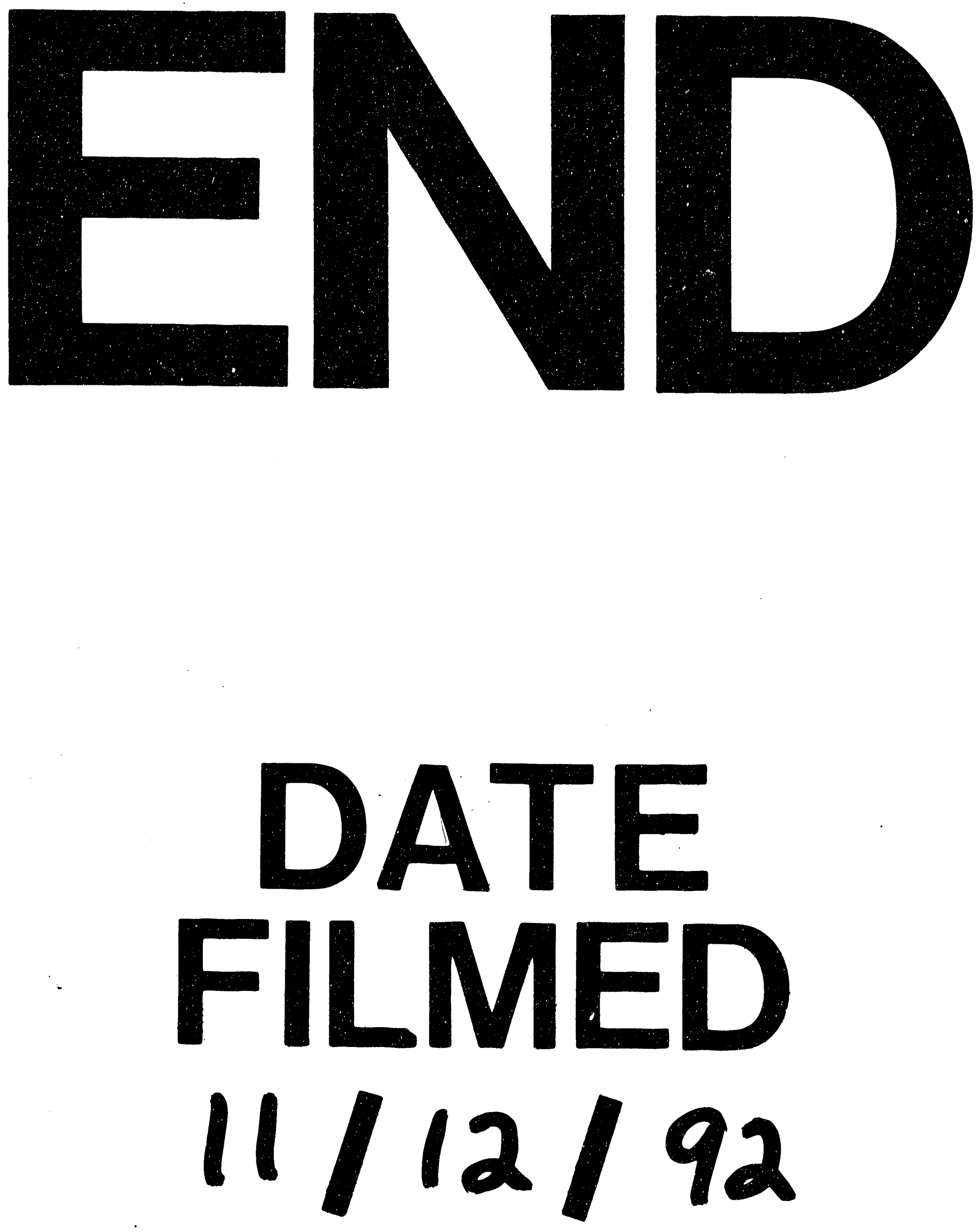

1 
